\title{
A Self-Contained Roadway Kinetic Energy Harvesting \\ System for Energy Recovery
}

by

Xinghe Wang

A thesis submitted to the Faculty of Graduate and Postdoctoral

Affairs in partial fulfillment of the requirements for the degree of

\author{
Master of Applied Science \\ in
}

Mechanical Engineering

Carleton University

Ottawa, Ontario

(C) 2015, Xinghe 


\begin{abstract}
Kinetic Energy Harvesting (KEH) systems have been applied to various maintenance-free electronic devices. One of the potential applications of such a system is harvesting a decent amount of kinetic energy generated by road vehicles. Although researches have been conducted on roadway KEH system, it is worthwhile to improve the system in order to convert more energy without worsening the driving experience. This work consists of design of a piezoelectric KEH transducer (Cymbal transducer), and improvement of the $\mathrm{KEH}$ circuit. The optimal geometry of each Cymbal unit is determined by the utilization of Finite Element Analysis. After studying several recently introduced piezoelectric KEH circuit topologies, a preferred topology has been adopted for a novel KEH circuit design. A voltage peak detection function and an energy storage unit voltage hysteresis function have been included in the circuit. The circuit has demonstrated a higher energy harvesting efficiency $(12.28 \%)$ compared to competitors during tests.
\end{abstract}




\section{Acknowledgements}

Firstly, I would like to express my sincere gratitude to my research supervisor, Prof. Jie Liu and Prof. Junjie Gu, for their continous encourangment and excellent academic guidance. It was they who led me into a brand-new research field that I found extremely interesting. The weekly research meetings with them are always instructive, and the research experience gained with them will benefit me for the rest of my life. In the meantime, I would like to express my appreciation to our industry partner, BW Services Inc., for their financial support to this research project.

My special thanks go to my research team member, Matteo Louter, who has always been by my side during the initial design, the tedious fabrication, and the chilly field tests. $\mathrm{He}$ has provided me with so much assistance, and the research experience would not be enjoyable without him. Many thanks should also be given to the Machine shop technologist, Alex Proctor, for his patient operation guidance during the fabrication process, and to the hydraulic load frame technologist, David Raude, who has spent a lot of time helping us do the loading tests, and his sense of humor is always pleasant.

Last but not least, I am very grateful to my parents in China for their endless love over these years, and for giving me the opportunity to have this precious overseas study experience. 


\section{Table of Contents}

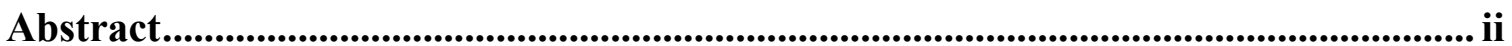

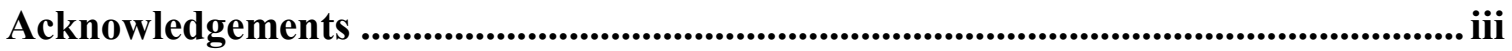

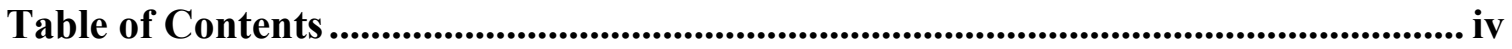

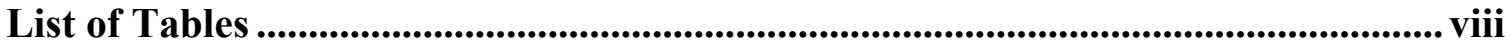

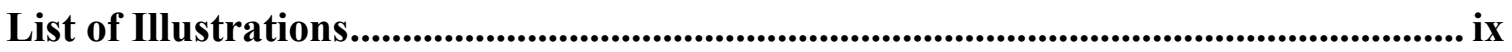

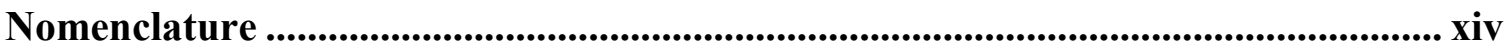

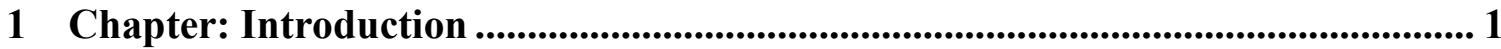

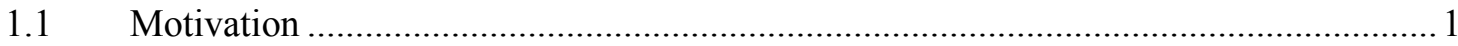

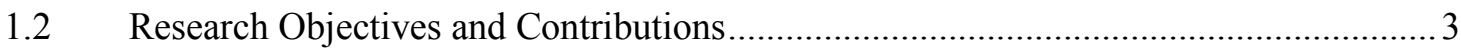

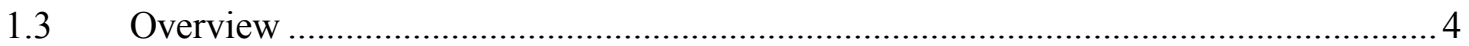

2 Chapter: Literature Review of Kinetic Energy Harvesting Transducer (KEHT) 6

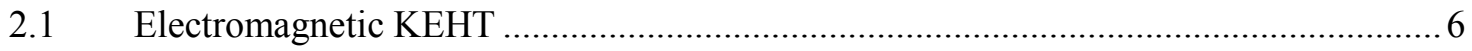

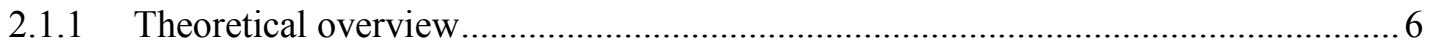

2.1.2 Electromagnetic KEHT on footwear................................................................ 7

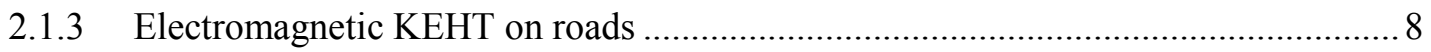

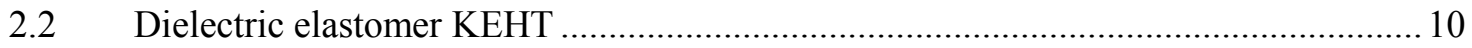

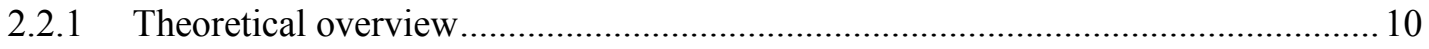

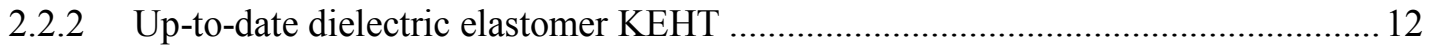

2.2.3 Dielectric elastomer KEHT on ocean waves..................................................... 13

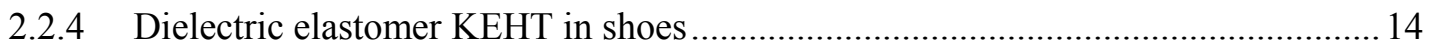

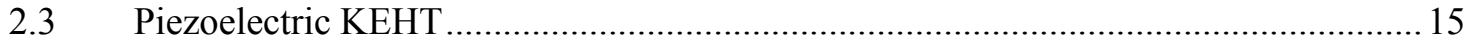

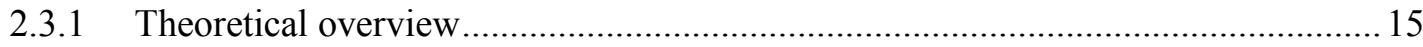

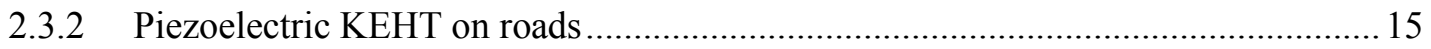




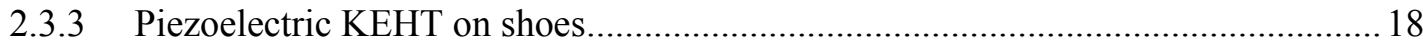

2.4 Applicability of different KEHTs to roadway energy harvesting ............................2

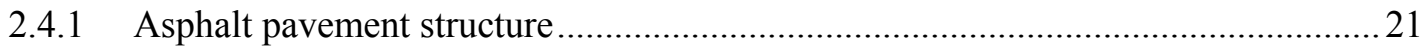

2.4.2 KEHs comparison for roadway energy harvesting..............................................2 22

3 Chapter: PZT Energy Transducer ............................................................................. 24

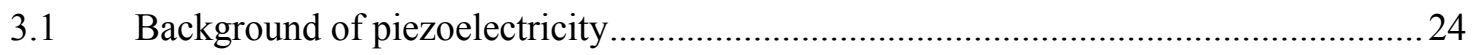

3.1.1 Direct piezoelectric effect and converse piezoelectric effect ...............................24

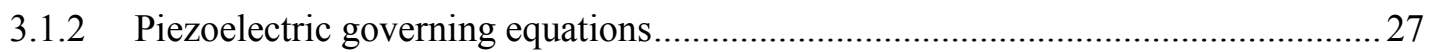

3.1.3 Piezoelectric coefficients in different modes of direct piezoelectric effect ..............30

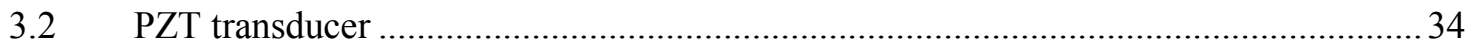

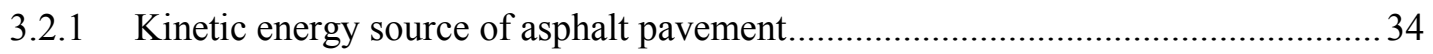

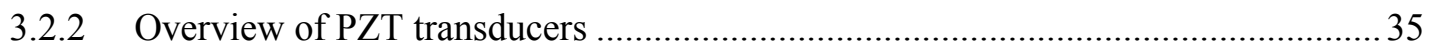

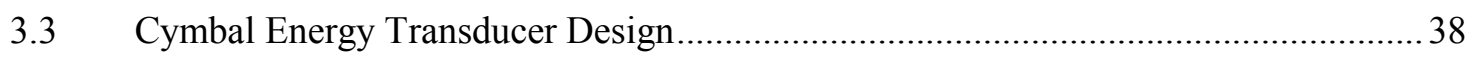

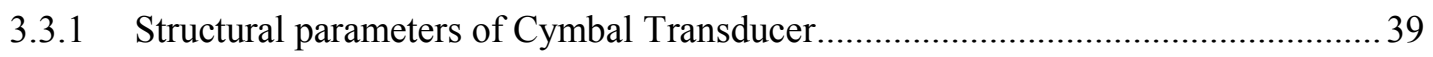

3.3.2 Analysis of Cymbal model under compression.................................................. 40

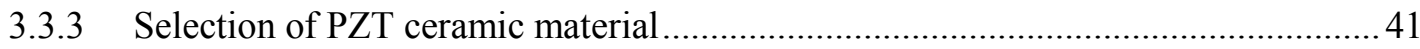

3.3.4 Selection of Cymbal transducer structural parameters........................................ 45

3.3.4.1 Finite Element Analysis (FEA) of the Cymbal transducer ...............................45

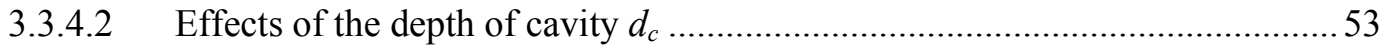

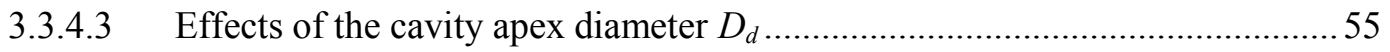

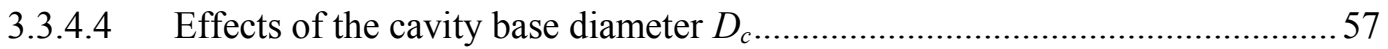

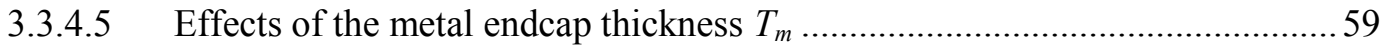

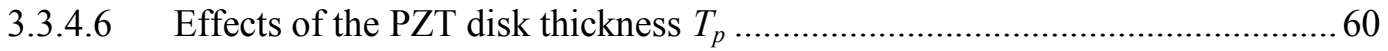

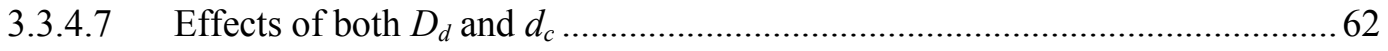

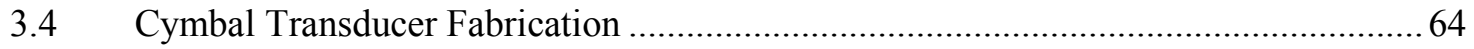

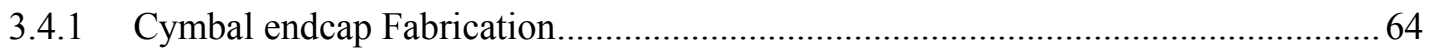




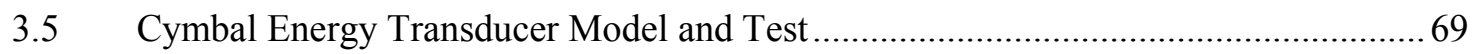

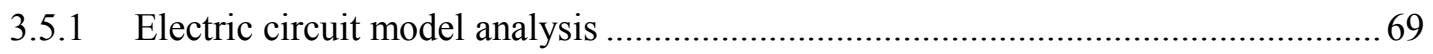

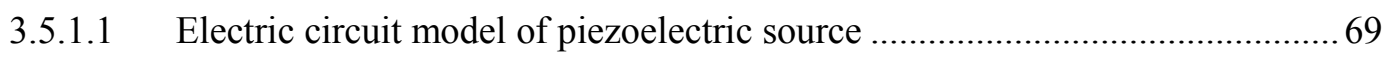

3.5.1.2 Electric circuit model of the open circuit test............................................ 73

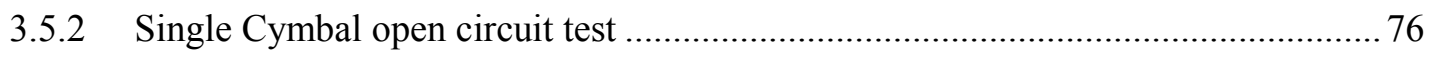

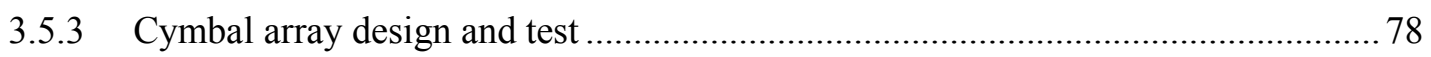

3.5.4 Energy harvesting speed bump design and test.............................................. 83

4 Chapter: KEH Circuit Topology Determination ................................................ 88

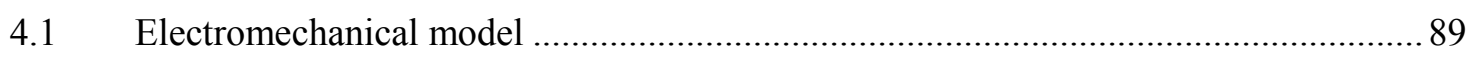

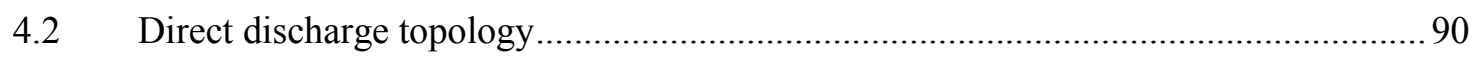

4.2.1 Power output of the direct discharge topology through a full-wave rectifier...........91

4.2.2 Power output of the direct discharge through half-wave rectifier...........................96

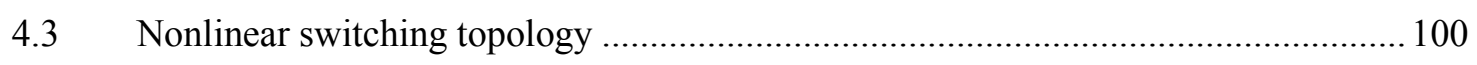

4.3.1 Synchronized switch harvesting on inductor (SSHI) topology ............................ 101

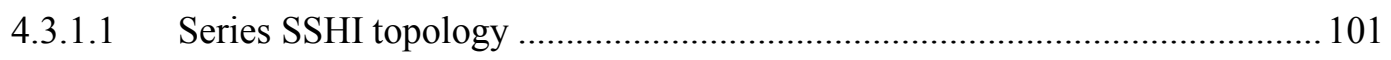

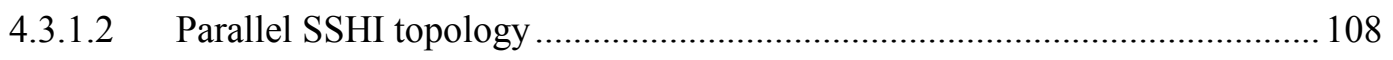

4.3.2 Synchronized Switching and Discharging to a storage Capacitor through an

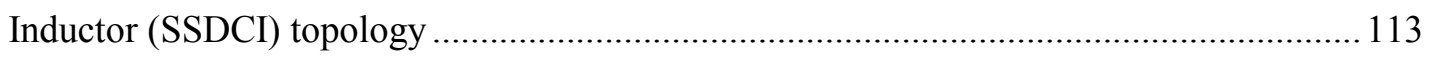

4.3.3 Synchronized Electric Charge Extraction (SECE) topology ................................ 120

4.3.4 Double Synchronized Switch Harvesting (DSSH) topology ................................ 125

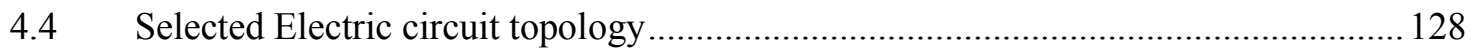

5 Chapter: Energy Harvesting Electric System ................................................. 132

$5.1 \quad$ Power Harvesting Circuit Design Considerations .................................................. 132

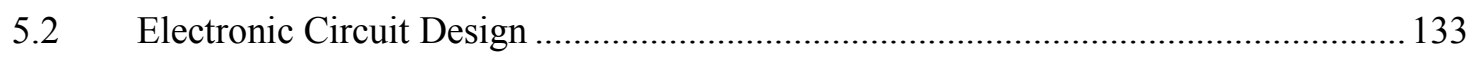

5.2.1 Piezoelectric voltage rectification and "cold start" ........................................... 135 


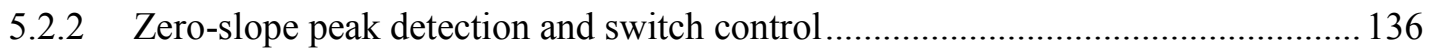

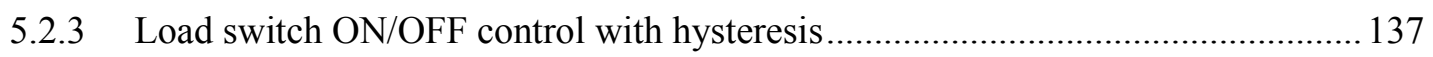

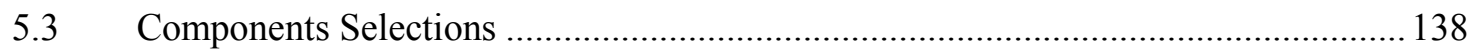

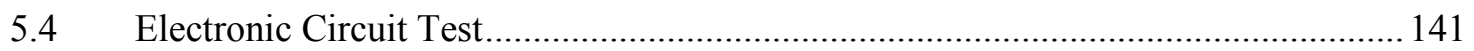

5.4.1 Test with the energy harvester on an hydraulic load frame .................................. 141

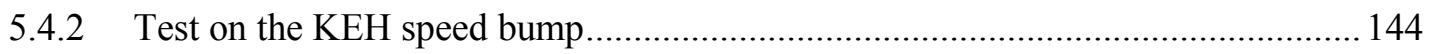

6 Chapter: Conclusions and Future work .......................................................... 147

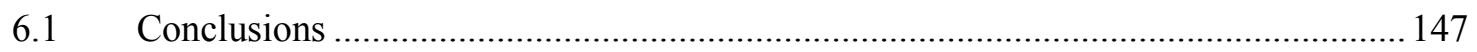

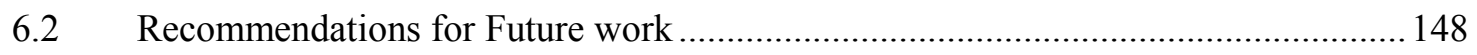

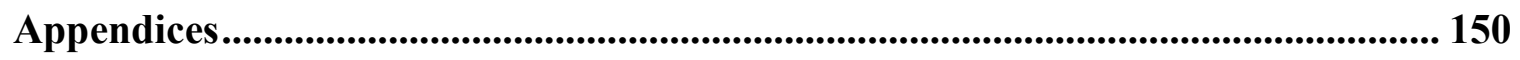

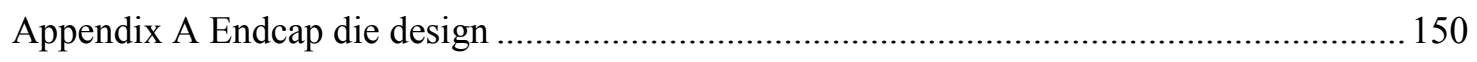

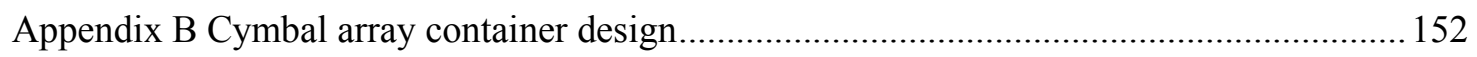

Appendix C Functional Description and Efficiency of the MIT Piezoelectric Power harvesting

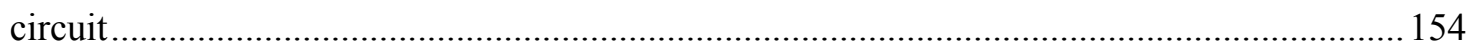

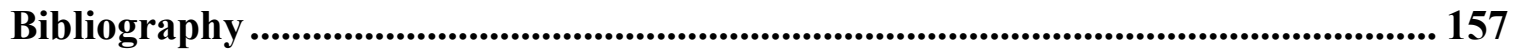




\section{List of Tables}

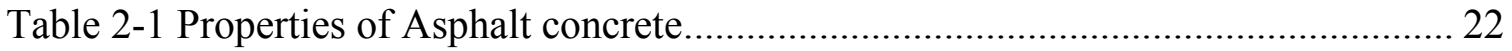

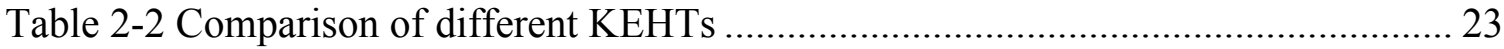

Table 3-1 Piezoelectric coefficients of three modes .................................................... 33

Table 3-2 Parameters of PZT materials of different Navy types................................. 44

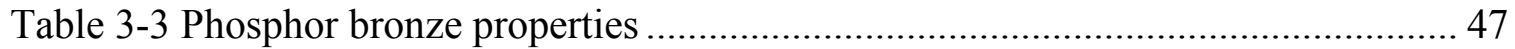

Table 3-4 Structural parameters used in FEA simulation ........................................... 47

Table 3-5 Comparison between the fine and the extra fine meshes. ............................ 48

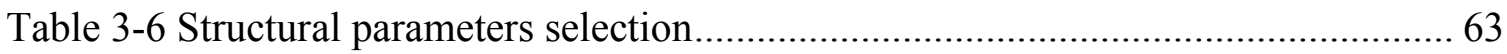

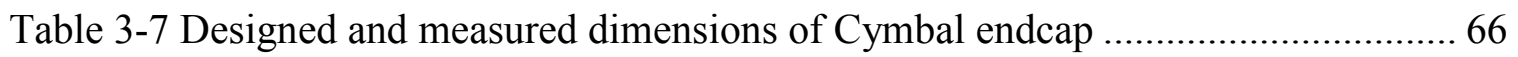

Table 5-1 Truth table for the comparators and the NAND latch ................................ 137

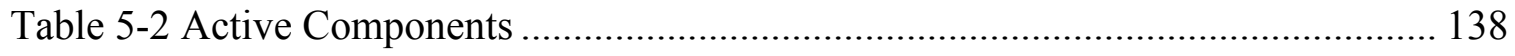

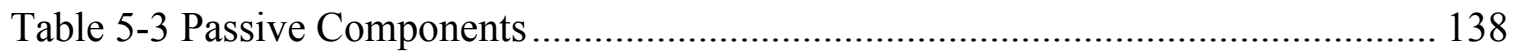




\section{List of Illustrations}

Figure 1.1 EHS schematic diagram. ................................................................. 3

Figure 2.1 (a) Schematic diagram of a DC generator; (b) The magnitude of the current [9]. 7

Figure 2.2 Magnetic generators embedded in a sneaker sole with gear box [11] ............ 8

Figure 2.3 Roadway KEHT by Pavegen Systems: (a) start position; (b) end position [12].

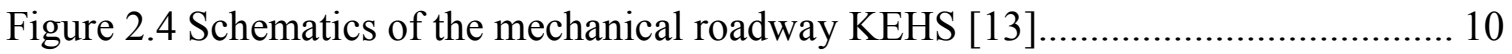

Figure 2.5 Schematic of an ideal Dielectric Elastomer energy cycle. .......................... 11

Figure 2.6 Dielectric elastomer KEHT at (a) plan view, (b) side view and (c) side view in

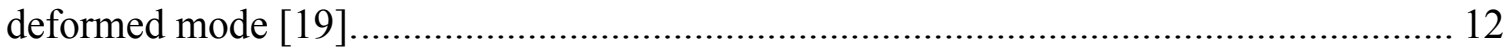

Figure 2.7 Two pairs of DEG oscillated 180 degrees out of phase [20]........................ 13

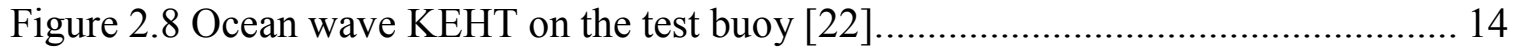

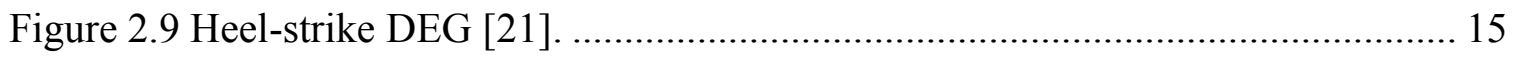

Figure 2.10 Cantilever mechanical configuration [23] ......................................... 16

Figure 2.11 FEA model of the Cymbal transducer buried in the asphalt concrete [24]... 17

Figure 2.12 Schematics of Innowattech energy harvesting container: (a) cross-section

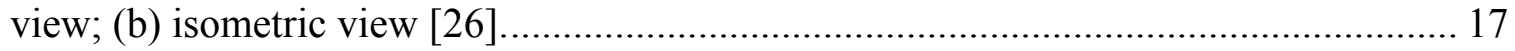

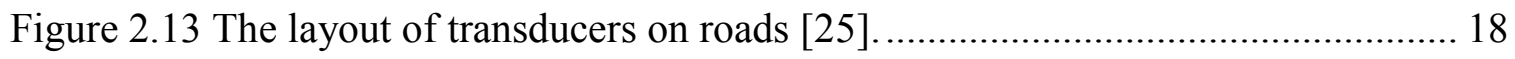

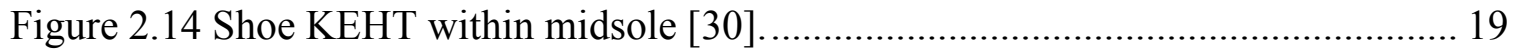

Figure 2.15 (a) Exploded view of the shoe harvester; (b) RFID circuit and piezoelectric

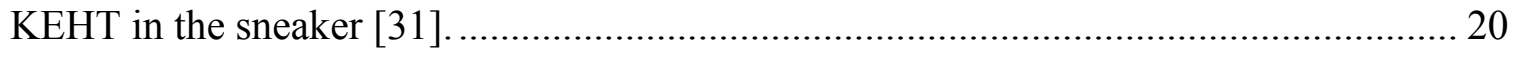


Figure 2.16 Rectangular piezoelectric bimorph inside the shoe [32]. 20

Figure 2.17 Structure of a typical asphalt pavement. 21

Figure 3.1 PZT perovskite crystal structure: (a) above Curie point, balanced positive and negative charges; (b) below Curie point, crystal has electric dipole moment [43].......... 25 Figure 3.2 Direct piezoelectric effect: (a) under compression; (b) under tension (dashed shape indicates initial state). 26 Figure 3.3 Converse piezoelectric effect: (a) under applied voltage same to the polarization direction; (b) under applied voltage opposite to the polarization direction

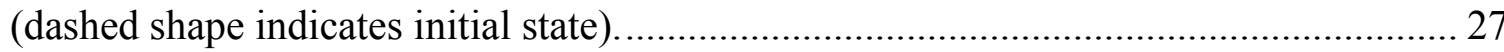

Figure 3.4 Possible stress directions of a piezoelectric element. ................................ 31

Figure 3.5 Three direct piezoelectric modes: (a) 33-mode; (b) 31-mode; (c) 15-mode... 31

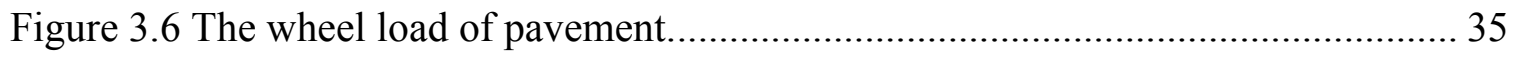

Figure 3.7 Typical configurations of PZT transducers: (a) Unimorph; (b) Bimorph; ...... 36

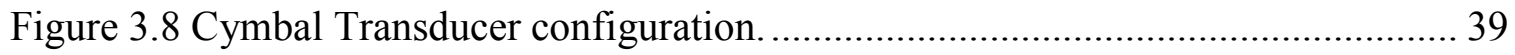

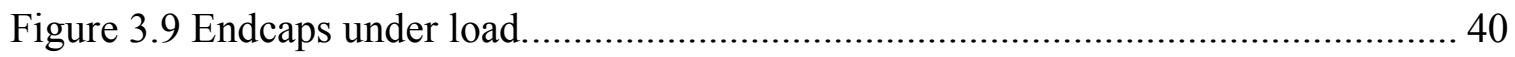

Figure 3.10 PZT disk under radial tensile stress and compressive stress. ..................... 42

Figure 3.11 Cymbal model in COMSOL Multiphysics............................................ 46

Figure 3.12 Von Mises Stress of Cymbal endcap: (a) Isometric view; (b) Cross-section view

Figure 3.13 (a) First principal stress (tensile); (b) Third principal stress (compressive).. 50 Figure 3.14 Shear stress $T_{31}$ distribution........ 51

Figure 3.15 Open circuit voltage generated in PZT ............................................. 52 
Figure 3.16 Effects of cavity depth $d_{c}$ : (a) Stress of PZT; (b) Displacement of rigid block and endcap; (c) Maximum VonMises Stress of the endcap; (d) Generated voltage and energy in PZT. 54

Figure 3.17 Effects of cavity apex diameter $D_{d}$ : (a) Stress of PZT; (b) Displacement of rigid block and endcap; (c) Maximum VonMises Stress of the endcap; (d) Generated

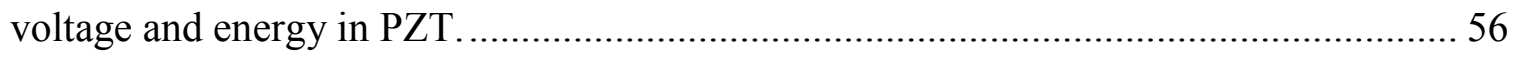

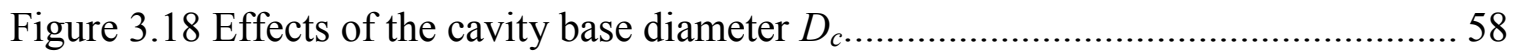

Figure 3.19 Effects of the metal endcap thickness $T_{m}$............................................... 59

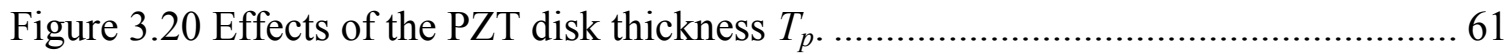

Figure 3.21 Stresses in Cymbal: (a) PZT maximum Tensile Stress; (b) PZT Central Tensile Stress; (c) Endcap Von Mises Stress; (d)PZT Maximum Shear Stress. .............. 63

Figure 3.22 Die set top section (left) and base section (right)....................................... 64

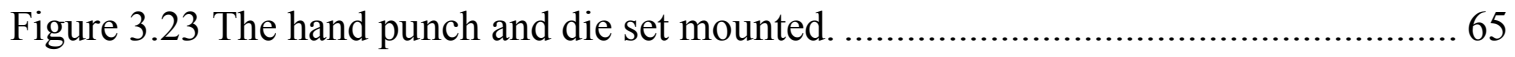

Figure 3.24 Force sweep of the measured model. ...................................................... 67

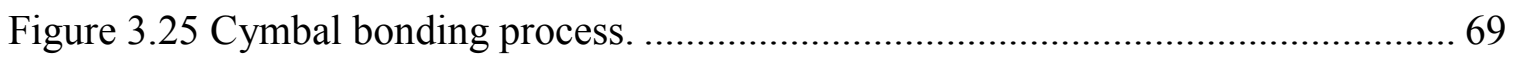

Figure 3.26 Equivalent electric circuit model of Cymbal transducer............................. 70

Figure 3.27 Waveforms of Cymbal displacement $u(t)$, velocity $v(t)$, open circuit voltage

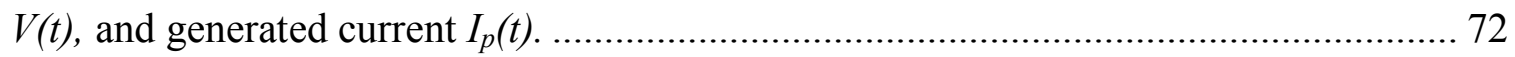

Figure 3.28 Equivalent circuit of open circuit voltage test............................................. 73

Figure 3.29 Voltage decaying due to the oscilloscope probe. ………………................ 75

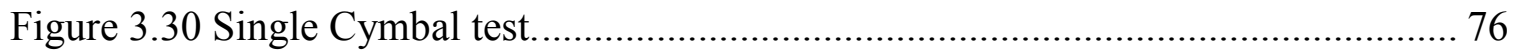

Figure 3.31 Open circuit voltage of Cymbal unit. ...................................................... 78

Figure 3.32 Cymbal array layout. …………………….............................................. 80 


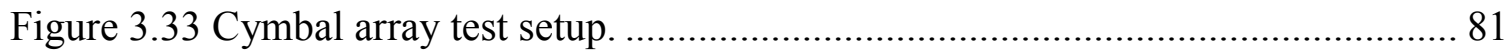

Figure 3.34 Open circuit Voltage output of Cymbal array............................................ 82

Figure 3.35 Speed bump embedded with Cymbal array.............................................. 84

Figure 3.36 Speed bump energy harvester test setup.................................................. 85

Figure 3.37 Open circuit voltage of the speed bump harvester. ....................................... 86

Figure 4.1 Electromechanical model of Cymbal. ...................................................... 89

Figure 4.2 Equivalent circuit of the direct discharge topology with full-wave rectifier... 91

Figure 4.3 Waveforms of piezoelectric current and voltage of direct discharge topology

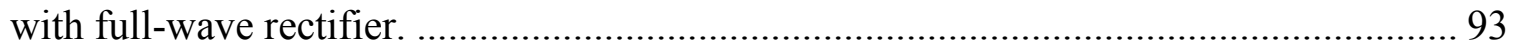

Figure 4.4 Energy increment in $C_{b}$ according to the rectified voltage $<V_{b}>\ldots \ldots \ldots \ldots \ldots . . . . .95$

Figure 4.5 Equivalent circuit of the direct discharge topology with half-wave rectifier. 96 Figure 4.6 Waveforms of piezoelectric current and voltage of direct discharge topology

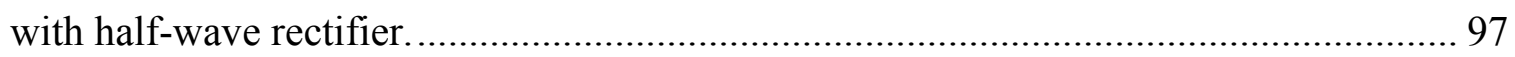

Figure 4.7 Energy increment in $C_{b}$ according to the rectified voltage $\left\langle V_{b}>\ldots \ldots \ldots \ldots \ldots \ldots . . .98\right.$

Figure 4.8 Electric circuit of the series SSHI topology ............................................. 102

Figure 4.9 Waveforms of piezoelectric current and voltage of the Series SSHI........... 103

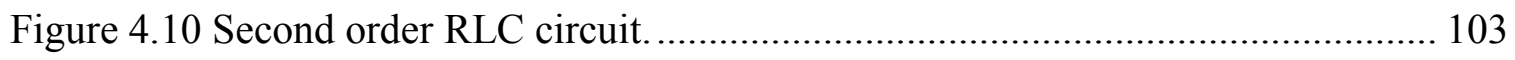

Figure 4.11 Energy increment in $C_{b}$ according to the rectified voltage $\left\langle V_{b}>\ldots \ldots \ldots \ldots . . .108\right.$

Figure 4.12 Electric circuit of the parallel SSHI.......................................................... 109

Figure 4.13 Waveforms of piezoelectric current and voltage of the Parallel SSHI....... 110

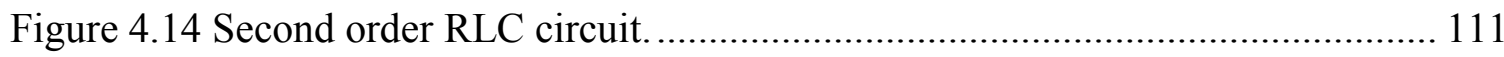

Figure 4.15 Energy increment in $C_{b}$ according to the rectified voltage $\left\langle V_{b}>\ldots \ldots \ldots \ldots . . .113\right.$

Figure 4.16 Electric circuit of the SSDCI topology....................................................... 114 
Figure 4.17 Waveforms of the SSDCI topology................................................... 115

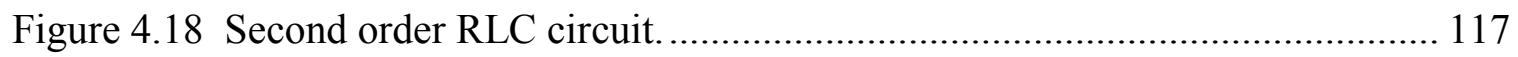

Figure 4.19 Energy increment in $C_{b}$ according to the rectified voltage $<V_{b}>\ldots \ldots \ldots \ldots . . .119$

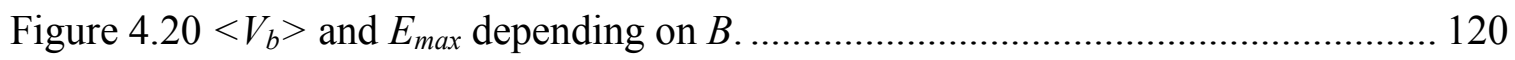

Figure 4.21 Equivalent electric circuit of the SECE topology........................................ 121

Figure 4.22 Waveforms of the SECE topology.................................................... 122

Figure 4.23 Energy increment in $C_{b}$ according to the rectified voltage $<V_{b}>\ldots \ldots \ldots \ldots . .124$

Figure 4.24 Equivalent electric circuit of the DSSH topology..................................... 125

Figure 4.25 Energy increment in $C_{b}$ according to the rectified voltage $\left\langle V_{b}>\ldots \ldots \ldots \ldots . . .128\right.$

Figure 4.26 The energy increment of different topologies during each load period with

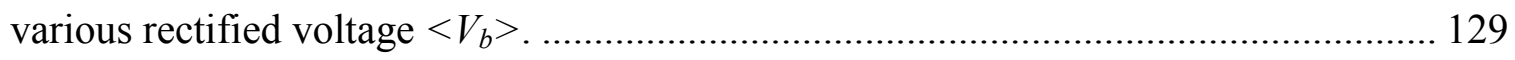

Figure 5.1 Piezoelectric energy harvesting circuit................................................... 134

Figure 5.2 Piezoelectric voltage and peak detection signal. ........................................ 142

Figure 5.3 Charging process of a $100 \mu \mathrm{F}$ capacitor.................................................... 143

Figure 5.4 Piezoelectric voltage and peak detection signal.......................................... 144

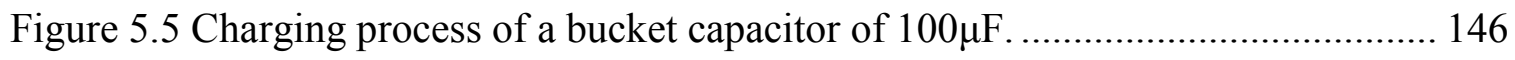

Figure A.1 Endcap die top part. ........................................................................ 150

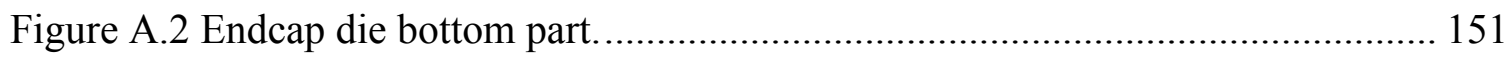

Figure B.1 The $3 \times 3$ "Cymbal” array container for MTS machine test........................... 152

Figure B.2 The $2 \times 5$ "Cymbal" array container for vehicle test.................................... 153

Figure C.1 Piezoelectric Power harvesting circuit from MIT media lab........................ 154

Figure C.2 300 $\mu \mathrm{F}$ bucket capacitor charging process under 36 steps............................ 155 


\section{Nomenclature}

KEHS

SRKEHS

KEHT

$\mathrm{KEH}$

FEA

PZT

PVDF

LED

EMF

DC

EAP

ISPC

MIT

RFID

RAINBOW

THUNDER

$\mathrm{CNC}$

SSHI

SSDCI

SECE

SSD

DSSH

WSN
Kinetic Energy Harvesting System

Self-contained Roadway Kinetic Energy Harvesting System

Kinetic Energy Harvesting Transducer

Kinetic Energy Harvesting

Finite Element Analysis

$\mathrm{Pb}$ (Lead) Zr (Zirconate) Ti (Titanate)

Polyvinylidene Fluoride

Light-Emitting Diode

Electromotive Force

Direct Current

Electroactive Polymer

Double Integrated Self-Priming Circuit

Massachusetts Institute of Technology

Radio Frequency Identification

Reduced and Internally Biased Oxide Wafer

Thin-layer composite unimorph ferroelectric driver and sensor

Computer Numerical Control

Synchronized Switch Harvesting on Inductor

Synchronous Switching and Discharging to a storage

Capacitor through an Inductor

Synchronized Electric Charge Extraction

Synchronized Switch Damping

Double Synchronized Switch Harvesting

Wireless Sensor Network 


\section{Chapter: Introduction}

\subsection{Motivation}

Energy shortage and greenhouse gas over-emissions are two of the most severe problems the world will be confronted with within the next few decades, because our current main energy source, fossil fuel, is unsustainable and its use is a cause of greenhouse effect. To solve this problem, a great amount of research work has been conducted to look for all kinds of alternative energy sources, such as thermal energy, electromagnetic energy, solar energy, kinetic energy, etc. These kinds of energy sources can be found all around us and usually will be dissipated into other forms of energy if not recovered in appropriate artificial ways [1, 2, 3, 4]. Among various ambient energies, kinetic energy is attracting more attention in the field of energy harvesting technology. Kinetic energy typically exists in the form of vibrations or random impacts, which are ubiquitous in our environment. It can be generated by human activities such as vehicle flow and pedestrian flow, or by natural phenomena such as ocean waves and river flows. In order to transfer the useless or wasted ambient kinetic energy into useful electric energy, a Kinetic Energy Harvesting System (KEHS) is necessary.

In addition to satisfying large-scale power requirements, KEHS can deal with micro power demands as well. With the rocketing development of computer hardware and electric engineering, hard disk drive capacity, CPU speed, available RAM have been increasing exponentially, as anticipated by Moore's law that the number of transistors in a dense integrated circuit doubles every two or three years. The technique development 
brings us smaller and smaller portable electric devices that consume negligible power (below $10 \mathrm{~mW}$ ). However, the development of the battery technology has encountered a bottleneck period, as the growth rate of the chemical battery energy density cannot keep up with the other hardware parameters [5]. Although Lithium-ion batteries have been widely used for most electronic devices, they commonly have a finite lifespan of only 500 to 1000 cycles. This means after a few years of usage, the battery in that portable device would be the first worn-out component. For those health detection devices planted in patients' bodies and those wireless sensors placed on structures like bridges and skyscrapers, it is troublesome and expensive to check and replace all the batteries one by one manually $[6,7]$; therefore, KEHS would be a promising battery substitute in these devices.

In 2014, there are over 4,000,000 $\mathrm{km}$ of paved roadways in America and over 400,000 $\mathrm{km}$ in Canada [8]. Millions of vehicles of all kinds are running on these roads every day, and the kinetic energy imposed on the roads are dissipated as heat energy. BW Services Inc., one of Canada's leading companies of facility renovations and repairs, initiated an ambitious research plan to develop a self-contained roadway kinetic energy harvesting system (SRKEHS). The system is expected to be able to harvest wasted kinetic energy from vehicles on the road, supply energy to some low-energy consuming electronic devices in those areas where power cable is unachievable and the battery maintenance is burdensome. 


\subsection{Research Objectives and Contributions}

A common KEHS is composed of energy transducer, voltage regulator and energy storage unit, as shown in Figure 1.1. Kinetic energy harvesting transducers (KEHT) are able to transfer specific ambient kinetic energy into reliable electric energy. For the sake of energy efficiency, the energy generated by the transducer usually cannot be directly connected to load. Thus, a voltage regulator is used to regulate the voltage to a satisfactory level to the load or store the energy for future usage in a storage unit, which could be a Lithium-ion battery, a capacitor or a supercapacitor. The energy storage unit is used as a buffer to store the energy before discharging to the load, considering the situation that the power consumption of the load is much larger than the average power generated by the energy transducer.

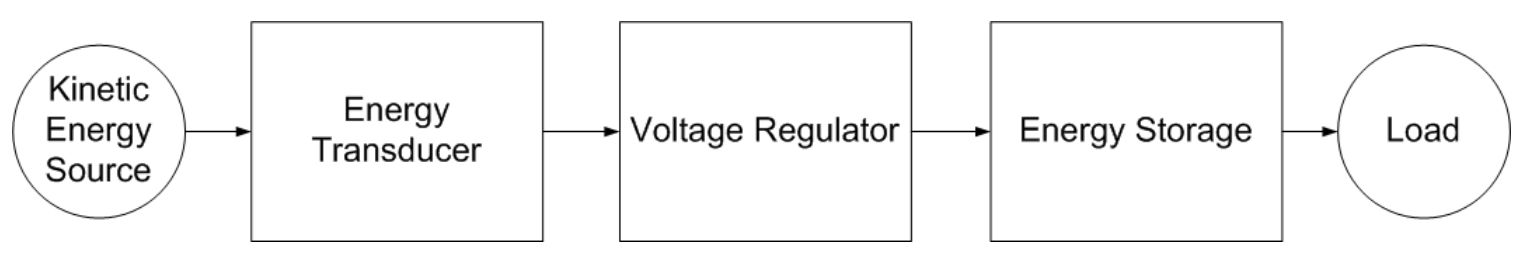

Figure 1.1 EHS schematic diagram.

Accordingly, a KEHS for roadway power harvesting should be composed of these three elements. Thus, the objectives of this thesis are to design a better KEHT suitable for roadway energy harvesting and improve the energy harvesting circuit to charge a proper energy storage unit more efficiently. The contributions of the thesis include:

- Comparison of different possible types of KEHTs suitable for harvesting kinetic energy from roads. The designed transducer that is applicable for energy 
harvesting under vehicles should endure heavy cyclic mechanical loads and should not worsen the driving experience.

- Design of a self-sufficient kinetic energy harvesting (KEH) circuit to charge an appropriate energy storage unit with higher efficiency. The voltage of the energy storage unit should be maintained in a range that is suitable for the load.

\subsection{Overview}

Chapter 2 gives a literature review of varied KEH devices using different types of transducers in recent years. It begins with the description and comparison of commonly used KEHTs such as electromagnetic KEHT, dielectric elastomer KEHT, and piezoelectric KEHT. For transducers using the same material, different shapes and different force amplification methods are compared as well. In this chapter, the reasons why we choose "Cymbal" with piezoelectric Lead Zirconate Titanate (PZT) ceramics as the KEHT on roads are presented.

Chapter 3 contains the basic theoretical background of piezoelectric material, and the design and manufacture process of the PZT transducer. Analytical modeling will be made for the Cymbal first to initially predict its performance with different geometries. A better and detailed Finite Element Analysis (FEA) using COMSOL Multiphysics is applied to determine the optimal geometry of the Cymbal unit. After that, the fabrication process of the Cymbal endcaps and the bonding process of the Cymbal are presented. After the discussion and comparison of different forms of Cymbal array connection 
methods, a square piezoelectric KEH container with an embedded Cymbal array is designed and tested in open circuit condition under a hydraulic load frame. Then a speed bump is modified with a rectangular piezoelectric KEH container embedded inside; the speed bump is tested in open circuit condition under a test vehicle. For each test, the total generated charge is calculated from the electric circuit model of the piezoelectric KEHT.

Chapter 4 gives the study of recent piezoelectric KEH circuit topologies. Their performance is compared in terms of the harvested energy during each load under various rectified voltages. The optimal rectified voltage is important as it determines how long the harvesting system takes to harvest the maximum energy in each load. Given the characteristics of the piezoelectric energy source and the load, the most appropriate topology is chosen.

In Chapter 5, a self-sufficient piezoelectric KEH circuit is modified from the chosen topology. A voltage peak detection function and an energy storage unit voltage hysteresis function are included in the circuit. The piezoelectric KEH circuit is tested with the square KEH container under the load frame and the KEH speed bump under a test vehicle. The efficiency of the circuit is calculated and compared with the circuit designed by the MIT media lab.

In Chapter 6, the conclusions and related future researches are summarized for the designed SRKEHS. 


\section{Chapter: Literature Review of Kinetic Energy Harvesting Transducer (KEHT)}

Typically, there are three candidates of possible KEHTs which could be utilized to harvest kinetic energy from roads: electromagnetic, dielectric, and piezoelectric transducers. In this chapter, different KEHTs and their applications are presented and compared, and one of them is determined to be the most suitable one for our SRKEHS.

\subsection{Electromagnetic KEHT}

\subsubsection{Theoretical overview}

The well-known electromagnetic generator is based on Faraday's law of electromagnetic induction discovered by Michael Faraday in 1831 [9]. Faraday found that if an electrical conductor moves through a magnetic field, a voltage potential difference will be generated between the conductor's ends. Faraday's law states that an electromotive force (EMF) will be produced by the interaction of a magnetic field and an electric circuit. Figure 2.1 illustrates the schematic model of a common electromagnetic KEHT, Direct Current (DC) generator. As the armature rotates, the conductive coils wound around the armature interact with the magnetic field and cause a current pulse to flow through the circuit. The commutator inverts the current direction every half cycle. In commercial DC generators, many more coils and commutators are distributed to generate a DC output superimposed by out-of-phase current pulses. 
Electromagnetic KEHT's advantages include its high efficiency and already wellestablished technology [10]. It is noted that on roadway, kinetic energy is provided by vehicles and the translation of road is in the vertical direction. Hence a mechanical coupling is necessary to transform the vertical translation into rotation motion of the generator's armature.

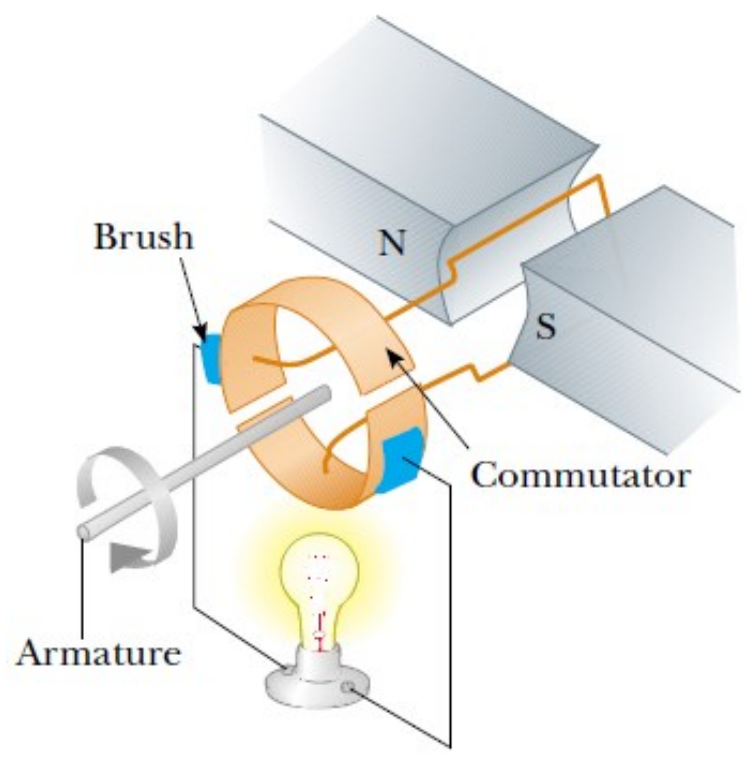

(a)

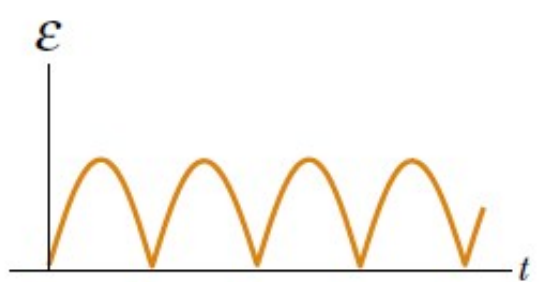

(b)

Figure 2.1 (a) Schematic diagram of a DC generator; (b) The magnitude of the current [9].

\subsubsection{Electromagnetic KEHT on footwear}

Jeffrey et al. (2000) developed a power harvesting footwear [11], which has a similar mechanical mechanism of a hand squeeze flashlight popular on the market. Shown in Figure 2.2, the KEHT in shoes is composed of two DC generators, a step-up gearbox, and is embedded in the sole of the sneaker. The hinged lever arm transfers the translation motion into rotation. Under walking heel strikes, the lever is compressed and rotates the 
armatures of the generators through a step-up gearbox and a belt transmission. The gearbox amplifies the rotary speed of the generators' armatures. A rotary clutch is used in the gearbox so that the lever arm is allowed to rotate in the opposite direction freely like flywheels, and solves the loss of the gears' inertia energy and lifespan while the heel is releasing. The KEHT is able to generate an average power of approximately $60 \mathrm{~mW}$ during normal walking process [11].

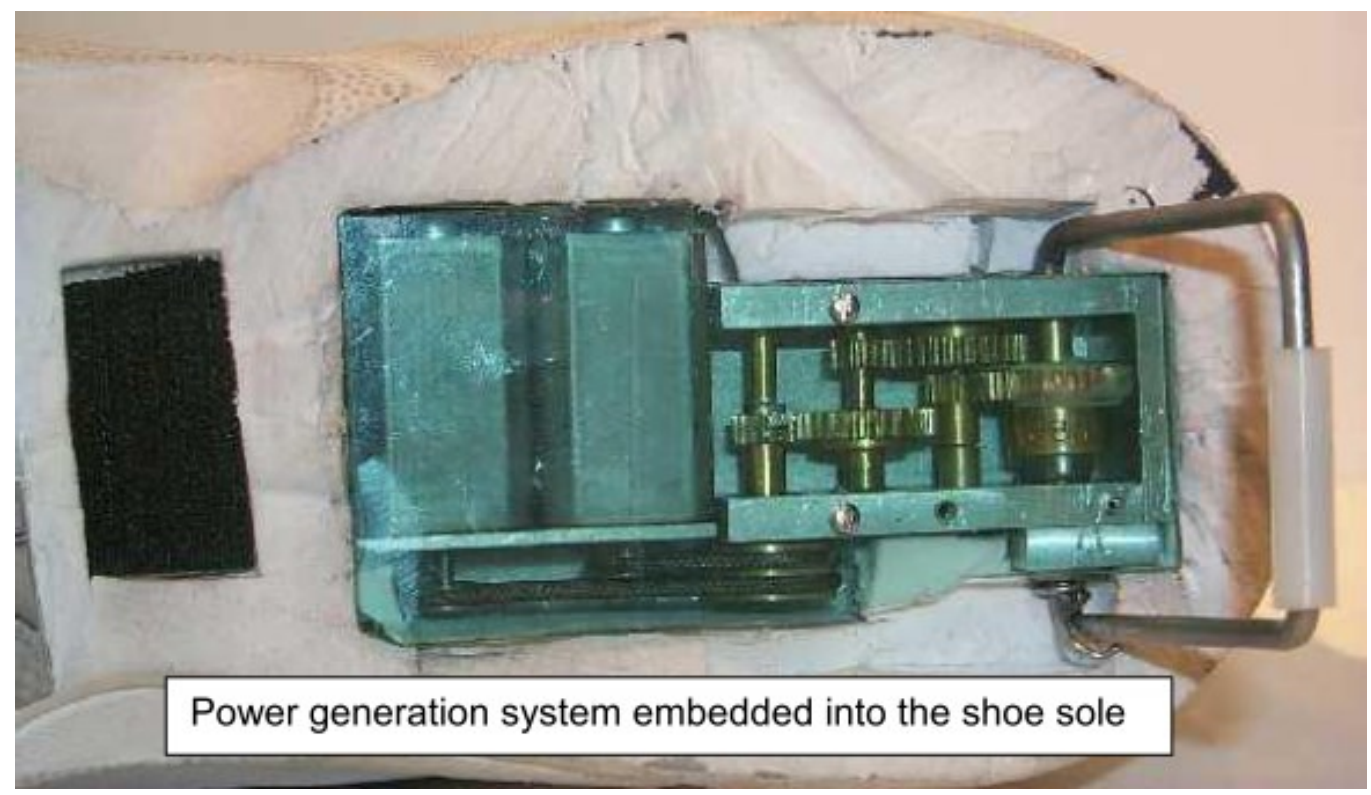

Figure 2.2 Magnetic generators embedded in a sneaker sole with gear box [11].

\subsubsection{Electromagnetic KEHT on roads}

Pavegen Inc. (England) has designed a roadway energy harvester for pedestrians and/or vehicles [12]. Figure 2.3 shows the internal schematics of the energy harvester. The top plate has three sets of engagement elements. Each element has a sequence of "teeth". When a pressure is applied on the top plate, these "teeth" will successively accelerate the rotation speed of the bottom plate, which has a number of magnets near its edge. In this 
way, downward translation is translated into rotational spin of the generator's rotor, which leads to the varying magnetic flux through copper coils located below the rotors. The electrical energy is used to power a Light-Emitting Diode (LED) in the demonstration. Pavegen Inc. claims that each generator is able to harvest 4-8 J of energy per compression, depending on the displacement of the top plate ( $4 \mathrm{~mm}$ to $7 \mathrm{~mm}$ ).

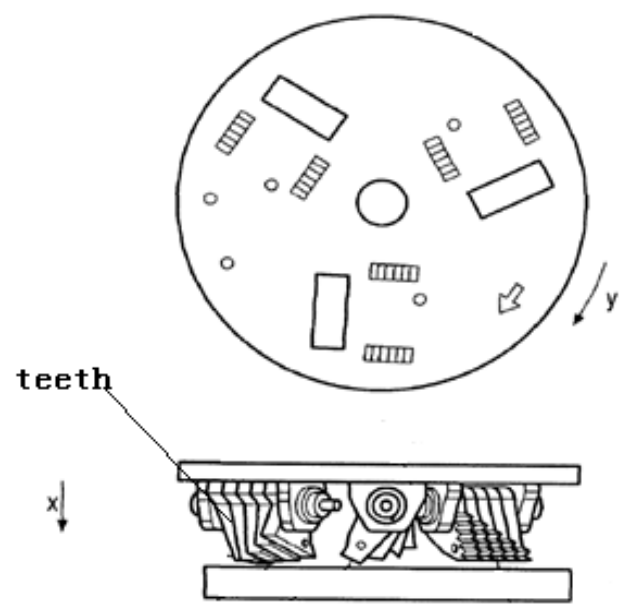

(a)

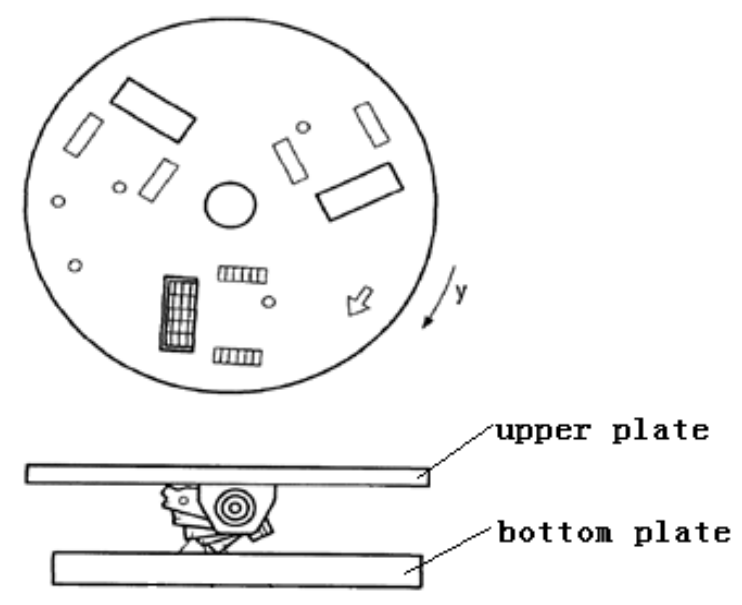

(b)

Figure 2.3 Roadway KEHT by Pavegen Systems: (a) start position; (b) end position [12].

Ting et al. (2011) has established a roadway vehicle KEH that uses electromagnetic KEHTs coupled by a hydraulic drive system [13]. As shown in Figure 2.4, when vehicles drive over the piston plates, the pistons ( $3 \mathrm{~cm}$ high) on the plates get pressed in sequence and drive fluid into the potential energy storage. Then, the potential energy storage supplies hydraulic fluid bilaterally into the hydraulic cylinder and push the piston in it back and forth, and the DC generator is rotated through a crank transmission. The oil reservoir is used to provide and store the hydraulic fluid in the system. The test shows the total working efficiency of the whole system reaches up to $41.03 \%$. Drawbacks of the 
system include bulky and expensive hydraulic devices, high maintenance cost, and possible hydraulic leakage problems. Since the pistons are $3 \mathrm{~cm}$ high, they may have a significant influence on the driving experience and slow down the vehicles greatly.

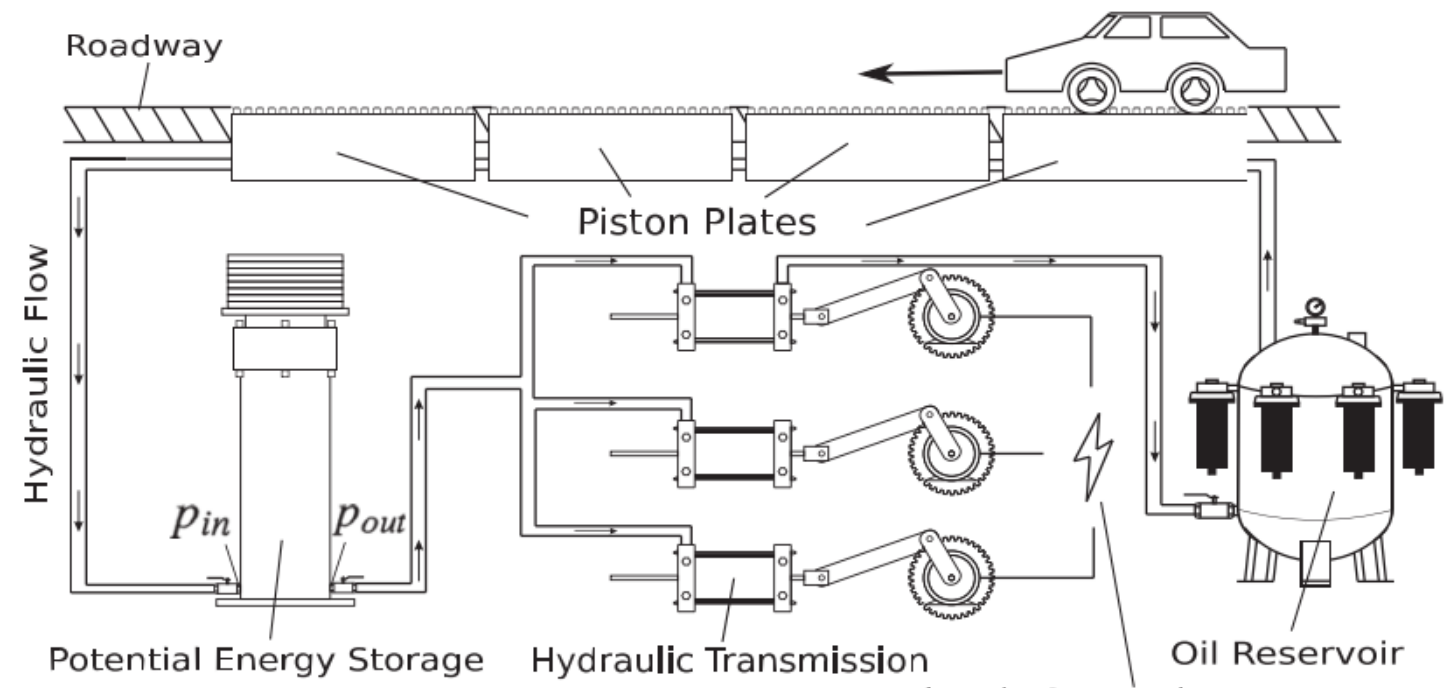

Electric Generation

Figure 2.4 Schematics of the mechanical roadway KEHS [13].

\subsection{Dielectric elastomer KEHT}

\subsubsection{Theoretical overview}

Dielectric elastomer is a type of electroactive polymer (EAP) that was initially used as artificial muscles for small bio-robots [14]. It exhibits a change in shape under an electric field and actuates large strains (over 300\%) [15]. Dielectric elastomer consists of a soft polymer, such as silicone or acrylic rubber, sandwiched between two compliant electrodes, so dielectric elastomer can be recognized as a deformable capacitor. The concept of dielectric elastomer is first given in a paper of Roentgen in 1880 [16]. He 
found a film of natural rubber can change in shape due to Columbic force if it is exposed to a strong electric field.

Dielectric elastomer can also be used in its generator mode if it works under an ideal energetic cycle (as shown in Figure 2.5) [17]. From the top left stage A, the dielectric elastomer is stretched by mechanical force and becomes thinner and wider. Then in stage B an electric field is applied to it, and the energy is stored inside the dielectric elastomer as electric charges $Q$. After that, in stage $\mathrm{C}$, the applied force is released, and the following deformation of the dielectric elastomer pushes unlike charges (on different surfaces) apart and like charges (on the same surface) together. The capacitance of the dielectric elastomer decreases from $C_{\max }$ to $C_{\min }$, and the voltage of the dielectric elastomer increases from $V_{\min }$ to $V_{\max }$. In the last stage $\mathrm{D}$, the energy stored in the dielectric elastomer is harvested. The energy generated from the mechanical elastic energy in each cycle can be calculated by $\frac{1}{2}\left(C_{\min } V_{\max }^{2}-C_{\max } V_{\min }^{2}\right)$. In this process, the mechanical energy stored in the dielectric elastomer is converted to electric energy.
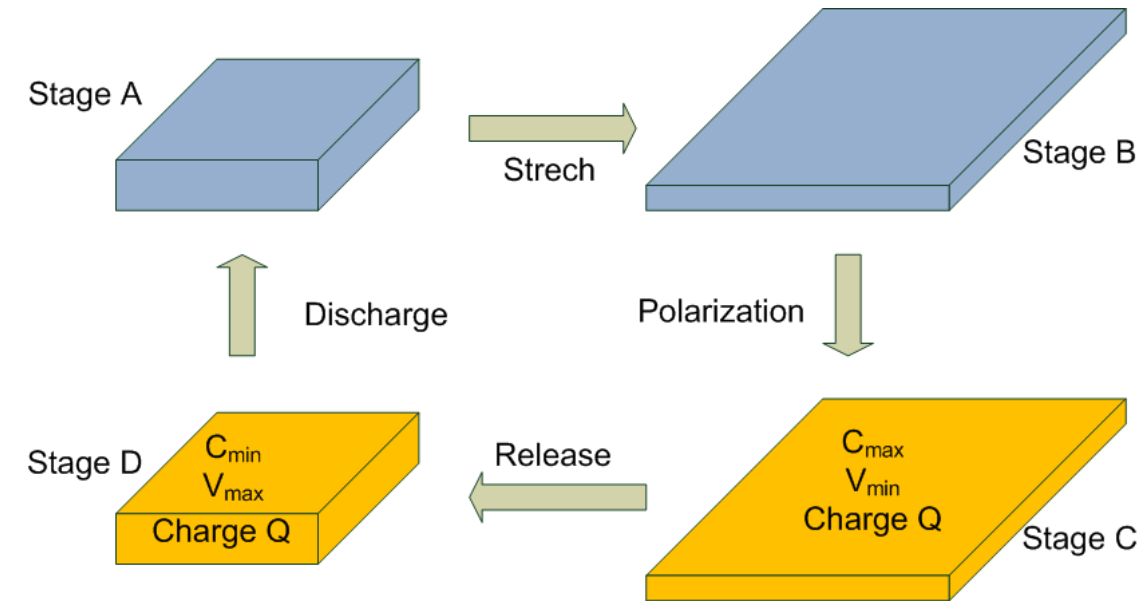

Figure 2.5 Schematic of an ideal Dielectric Elastomer energy cycle. 


\subsubsection{Up-to-date dielectric elastomer KEHT}

Apparently, in order to harvest energy from dielectric elastomer, an exterior high voltage source is required to place charges on the dielectric elastomer film during the first stage of each energetic cycle. To relieve the requirement of the high voltage source, McKay at el. (2010) developed a dielectric elastomer KEHT, as presented in Figure 2.6, and a selfpriming circuit that only needs a low voltage source to do the initial priming in the first energy cycle [18]. From the second cycle, the polarization charges are provided from the cycle before.

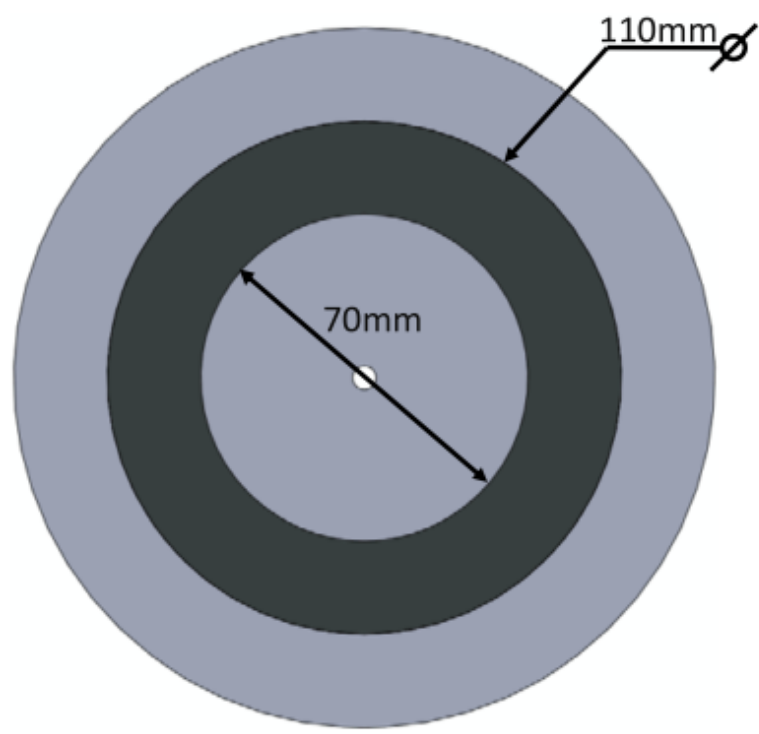

(a)

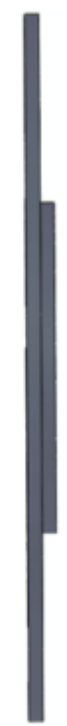

(b)

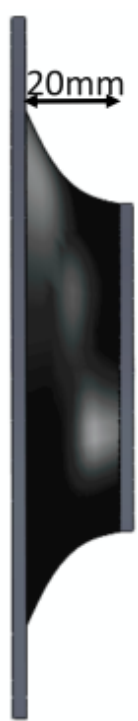

(c)

Figure 2.6 Dielectric elastomer KEHT at (a) plan view, (b) side view and (c) side view in deformed mode [19].

The self-priming harvesting circuit was then improved by the same research team to the double Integrated Self-Priming Circuit (ISPC) [20]. In ISPC, the dielectric elastomer generator is a Flat Trench Type [21], composed of two pairs of dielectric elastomer 
materials (Figure 2.7), whose internal capacitance is used as the capacitor banks in the previous harvesting circuit. In this way, the self-priming circuit can be simplified to only six diodes.

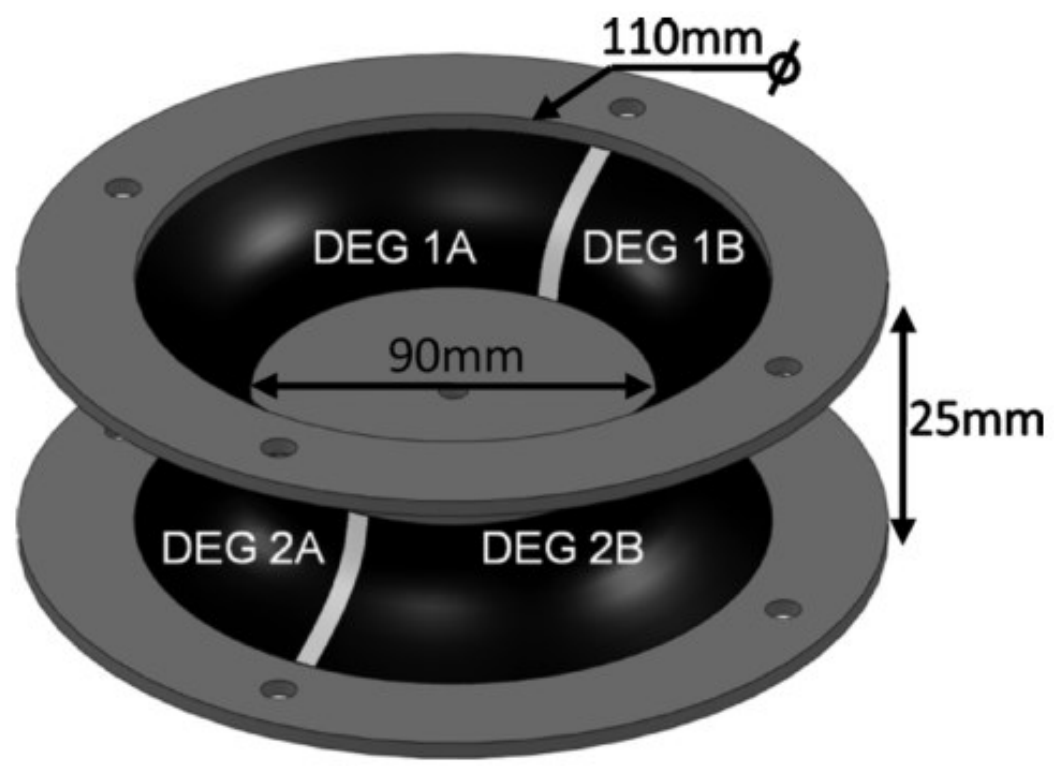

Figure 2.7 Two pairs of DEG oscillated 180 degrees out of phase [20].

\subsubsection{Dielectric elastomer KEHT on ocean waves}

The flexible property of the dielectric elastomer material makes it suitable for harvesting kinetic energy from motions of large displacement. In August 2007, SRI International and Hyper Drive (Japan) conducted a two-week's trial of a dielectric elastomer KEHT of wave-power at the coast of St. Petersburg, Florida, in Tampa Bay [22]. In Figure 2.8, the dielectric elastomer KEHT is connected and suspended on the top of a disk type navigation buoy and is attached with a mass of $62 \mathrm{~kg}$. The mass stretches and contracts the dielectric elastomer KEHT when the buoy heaves up and down on the ocean surface. In this trial, all the power generated by the dielectric elastomer KEHT buoy is measured 
by an electric measurement circuit powered by a battery. The results show that on a small wave height of $10 \mathrm{~cm}$, the dielectric elastomer KEHT generates an average power of $2.2 \mathrm{~W}$ and a peak power of $11 \mathrm{~W}$.

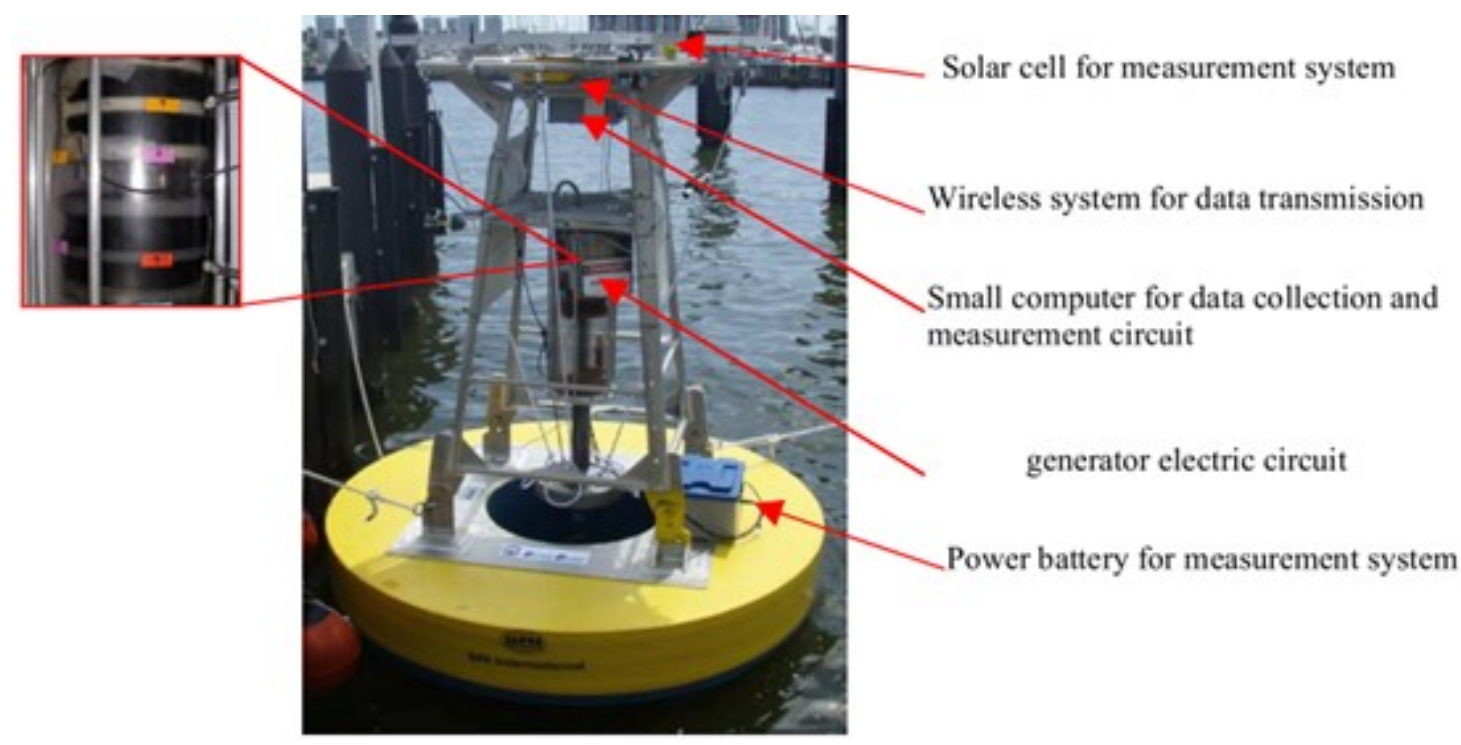

Figure 2.8 Ocean wave KEHT on the test buoy [22].

\subsubsection{Dielectric elastomer KEHT in shoes}

In 2011, SRI International developed a heel-strike KEHT using dielectric elastomer material [21]. The heel-strike KEHT does not give any extra burdens to the wears and is very light due to the low density of either the dielectric elastomer materials or the coupling medium. It is a diaphragm type of dielectric elastomer KEHTs. The polymer diaphragm in the dielectric elastomer KEHT is made of 20 stacked layers of dielectric elastomer films. Given the compression from the heel, the coupling medium of fluid or gel in the sole will squeeze the dielectric elastomer films out through the holes of the rigid plastic plate. Then the dielectric elastomer films are deformed. According to the paper, this device is able to generate $0.8 \mathrm{~J}$ of energy per footstep. 

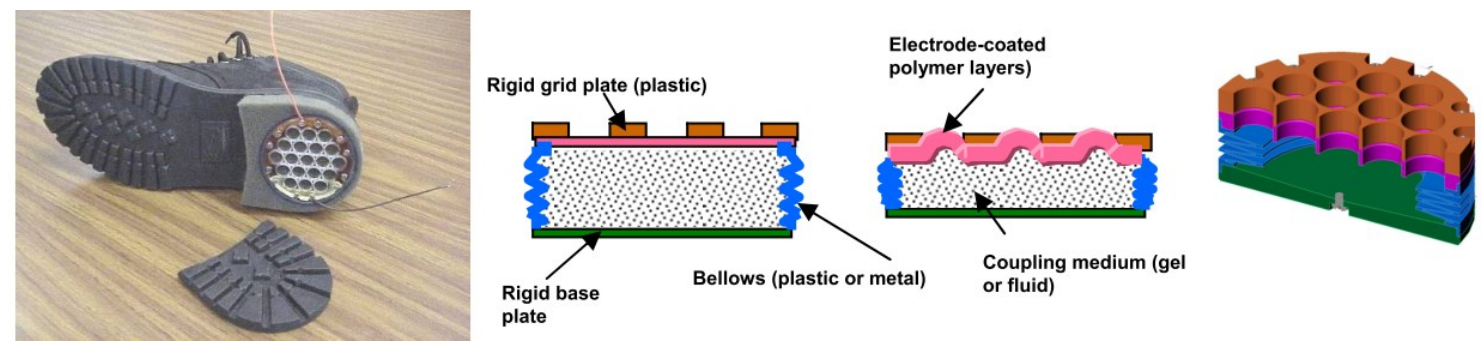

Figure 2.9 Heel-strike DEG [21].

\subsection{Piezoelectric KEHT}

\subsubsection{Theoretical overview}

Piezoelectric materials have two inherent effects: the direct piezoelectric effect that converts mechanical energy to electrical energy and the converse piezoelectric effect that converts electrical energy to mechanical energy. Piezoelectric KEHTs make use of the direct piezoelectric effect. When piezoelectric materials are under pressure or tension, certain amount of electric charges will be produced on its surface, and if the pressure or tension is released, piezoelectric materials will generate charges in the opposite direction. Detailed theoretical background and electrical model of piezoelectric materials will be presented in Chapter 3. Currently, there are two types of technologies that harvest energy from piezoelectric materials: the first one harvests energy from the vibrational kinetic source, the second one harvests energy from random force and impact.

\subsubsection{Piezoelectric KEHT on roads}

Messineo et al. (2011) designed a KEHT on roads using PZT cantilever transducers in a steel box [23]. The transducer is made of a cantilever beam with piezoelectric materials bonded on its surface. The beam is clamped at one end and free on the other end with a proof mass, which is used to adjust the resonant frequency of the cantilever. The springs 
underneath the box allow it to vibrate freely under the road. When the box is pressed by vehicles, it will cause the cantilever inside to vibrate. Then, the piezoelectric material on it is compressed and stretched during each cycle. The beam will continue to generate power for a period of time as the vibration of the cantilever decays.
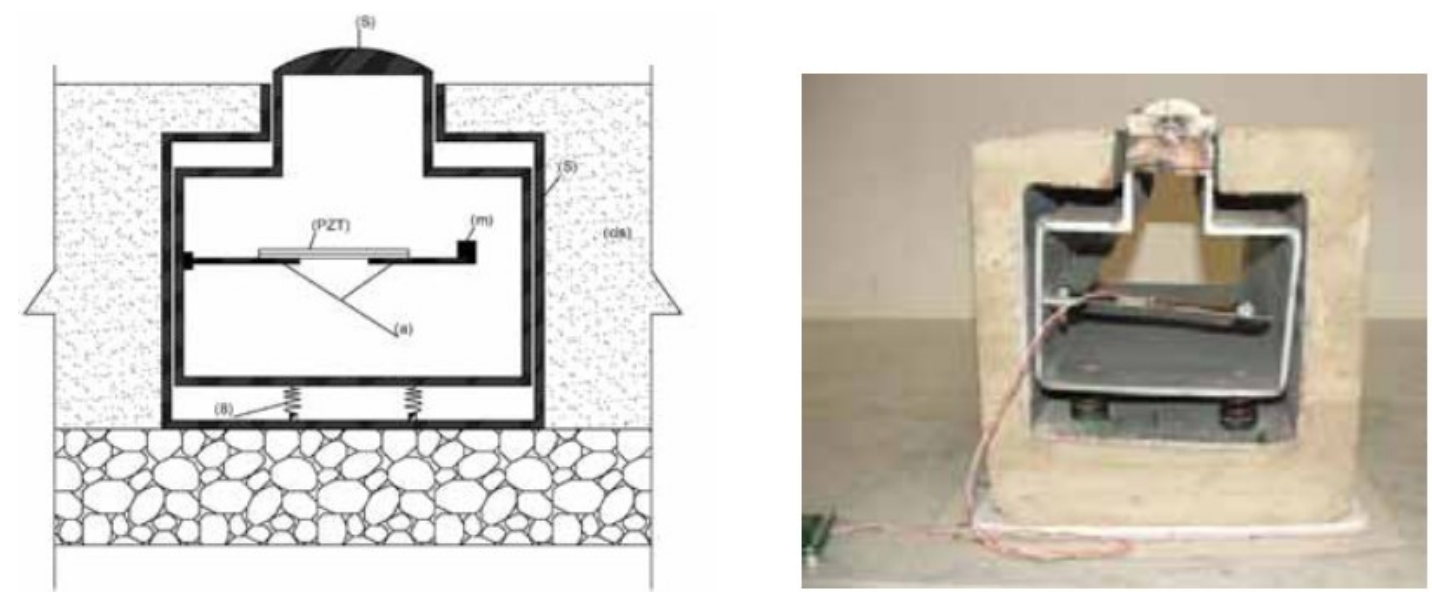

Figure 2.10 Cantilever mechanical configuration [23].

Zhao et al. (2010) designed a Cymbal transducer buried in the asphalt concrete to harvest the roadway kinetic energy [24]. The Cymbal transducer is made of one piezoelectric disk clamped by two metal endcaps. By adjusting the dimensions of the Cymbal endcaps, their effects on the pavement surface displacement can be reduced. 


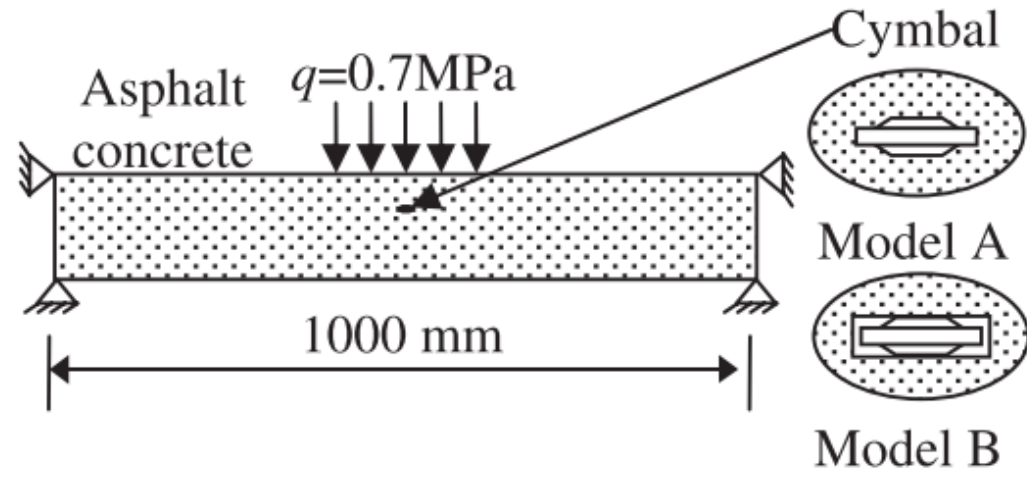

Figure 2.11 FEA model of the Cymbal transducer buried in the asphalt concrete [24].

Innowattech Inc. is a leading company in energy harvesting on roads, and it developed a standalone energy and data harvesting system [25]. It has three versions of different platforms: one can be installed on roads [26], one on airport runways [27], and one on railways [28]. Each version uses the same piezoelectric KEHTs, which are stacks of PZT ceramic disks, and they are organized and connected in arrays contained in a waterproof container as shown in Figure 2.12.

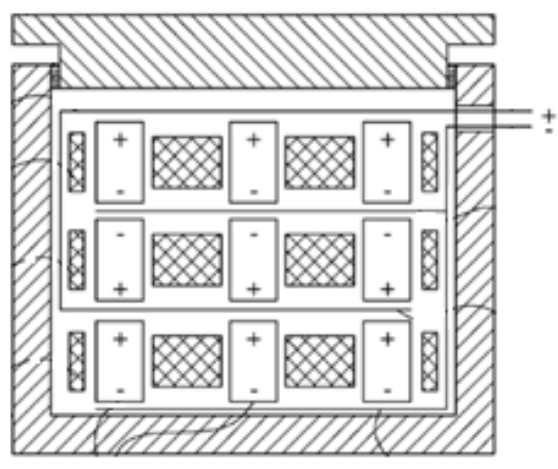

(a)

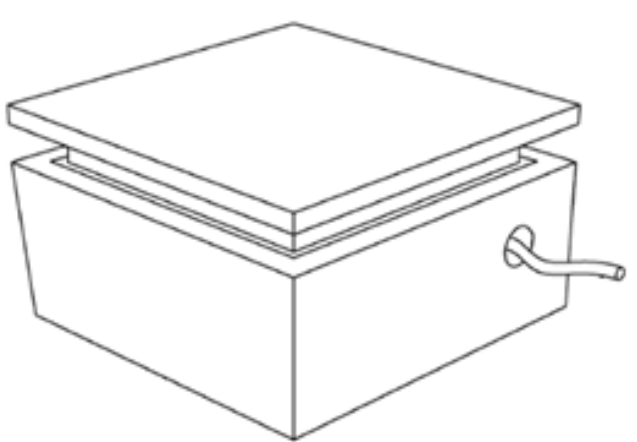

(b)

Figure 2.12 Schematics of Innowattech energy harvesting container: (a) cross-section view; (b) isometric view [26]. 
For asphalt pavement, the system is installed $5 \mathrm{~cm}$ below the surface of the pavement, beneath the upper asphalt layer. Innowattech claims that for 1 kilometer lane of pavement the harvesters embedded along two wheel's footprints, as shown in Figure 2.13, can generate $200 \mathrm{KWH}$ of energy per hour, assuming that 600 heavy vehicles drives through per hour with an average speed of $72 \mathrm{~km} / \mathrm{h}$ [25]. Some of the transducers can also be used as sensors to get data of the traffic flow, such as vehicles' weight, speed, their distance in between, etc. In 2010, Innowattech got a contract from Impregilo SpA, an Italian infrastructure and civil engineering company, to install power generators on the VeniceTrieste highway in Italy. The power generated on roads is used to power road signs [29].

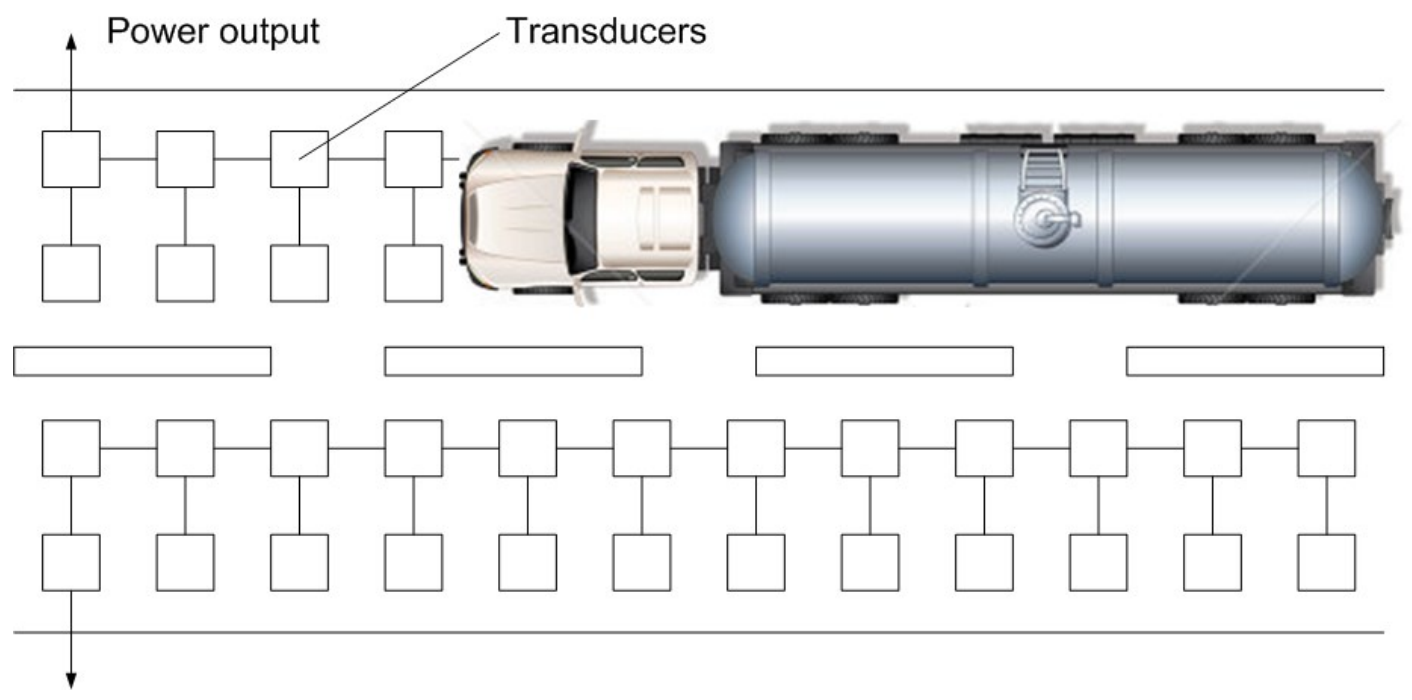

Figure 2.13 The layout of transducers on roads [25].

\subsubsection{Piezoelectric KEHT on shoes}

Antaki et al. (1995) designed a KEHT in shoes to power artificial organs [30]. As shown in Figure 2.14, there are two longitudinal PZT stacks in the midsole. The pressure from the forefoot and heel is amplified by the hydraulic amplifiers located at each end. In this 
design, $10 \mathrm{~mm}$ displacement of either toe or heel will cause $0.29 \mathrm{~mm}$ displacement of the PZT stacks. The disadvantage of this design is that the whole system, including transducers, related chassis, linkages, and metallic hydraulic couplings weighs a lot, and wearer's gait will be affected.
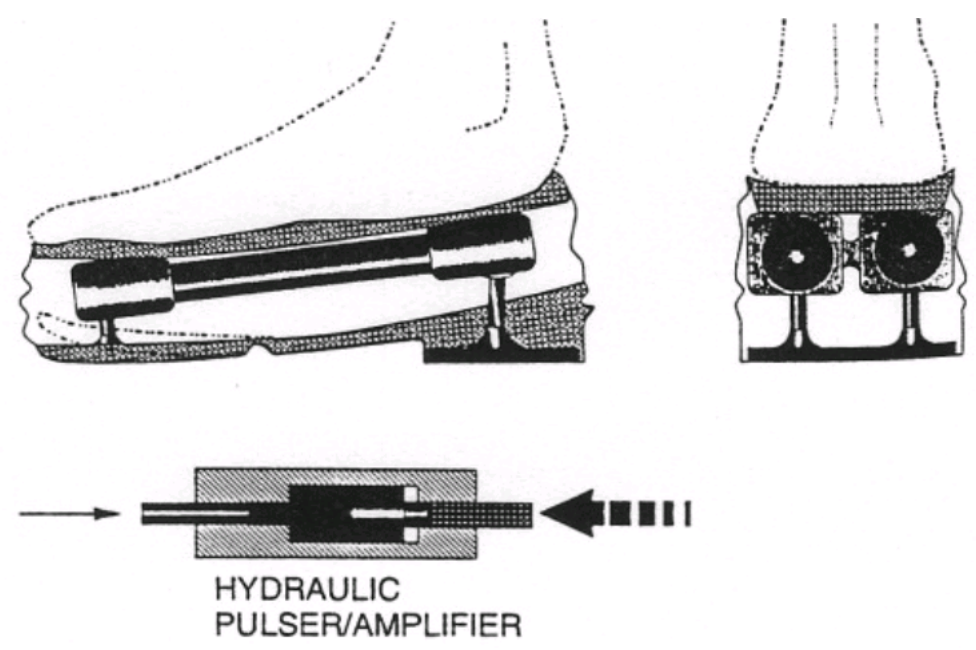

Figure 2.14 Shoe KEHT within midsole [30].

Massachusetts Institute of Technology (MIT) Media Laboratory of Cambridge, MA (1998) developed a lighter shoe harvester using a PZT unimorph and a Polyvinylidene Fluoride (PVDF) stave [31]. In Figure 2.15, both the PZT unimorph and the PVDF stave are installed between the rubber sole and the removable insole. The PZT unimorph is used to harvest energy from high-pressure heel strikes, and the softer PVDF stave is used to harvest energy from relatively low-pressure forefoot strikes. The test shows that under a $1 \mathrm{HZ}$ brisk walk, the PVDF stave will generate a $60 \mathrm{~V}$ pulse on a $250 \mathrm{k} \Omega$ load, and the PZT unimorph will generate around $150 \mathrm{~V}$ on the same load. The shoe harvester has been 
demonstrated to power a radio frequency identification (RFID) tag system to work for 0.5 second every 5 to 6 footsteps.

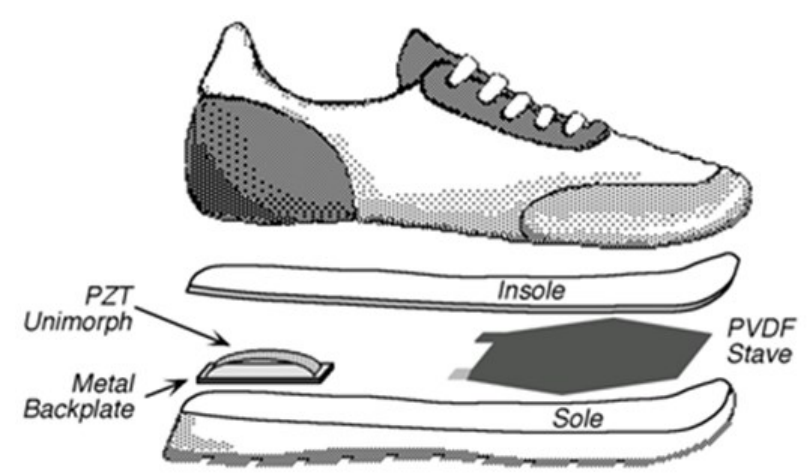

(a)

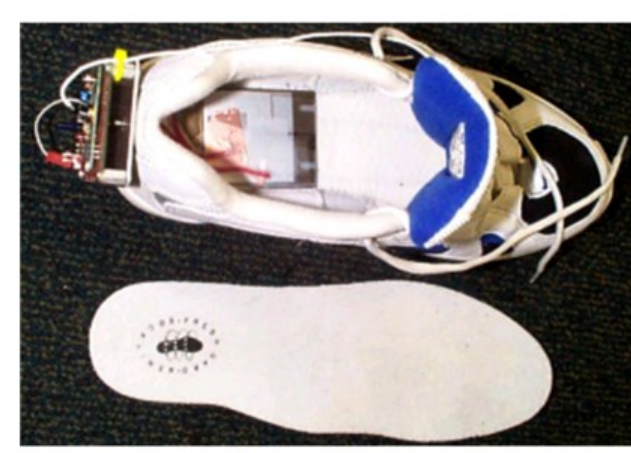

(b)

Figure 2.15 (a) Exploded view of the shoe harvester; (b) RFID circuit and piezoelectric KEHT in the sneaker [31].

Moro et al. (2010) designed a shoe-mounted piezoelectric cantilever to harvest the kinetic energy from footsteps [32]. Figure 2.16 illustrates how the piezoelectric beam is embedded in the insole. The piezoelectric beam is excited by the heel accelerations during human gait.

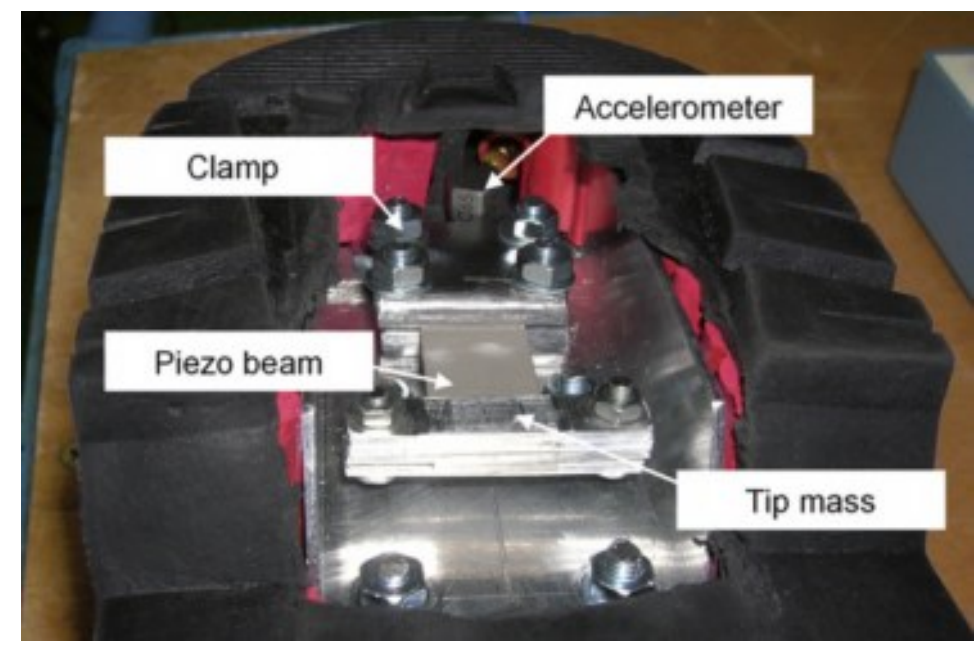

Figure 2.16 Rectangular piezoelectric bimorph inside the shoe [32]. 


\subsection{Applicability of different KEHTs to roadway energy harvesting}

\subsubsection{Asphalt pavement structure}

The properties of the asphalt pavement are essential for choosing the most suitable KEHT. A typical asphalt pavement is consisted of several layers (in Figure 2.17) including subgrade, subbase, base and surface from the bottom to the top [33, 34]. The subgrade is filled at the bottom to support the road. On top of the subgrade, the subbase is used to prevent the fines from intruding into the base and improve drainage. The base layer is made of multiple layers of asphalt concrete and is the most possible place where the KEHT will be installed. Then the base layer is covered with a thin layer $(50 \mathrm{~mm})$ of porous asphalt wearing course. Asphalt concrete is a visco-elastic material, which means that it has both elastic and viscous behaviors. Its Young's elastic modulus changes greatly according to the temperature. Table 2-1 shows the Young's modulus and Poisson's ratio of typical asphalt concrete and their relationship with the temperature.

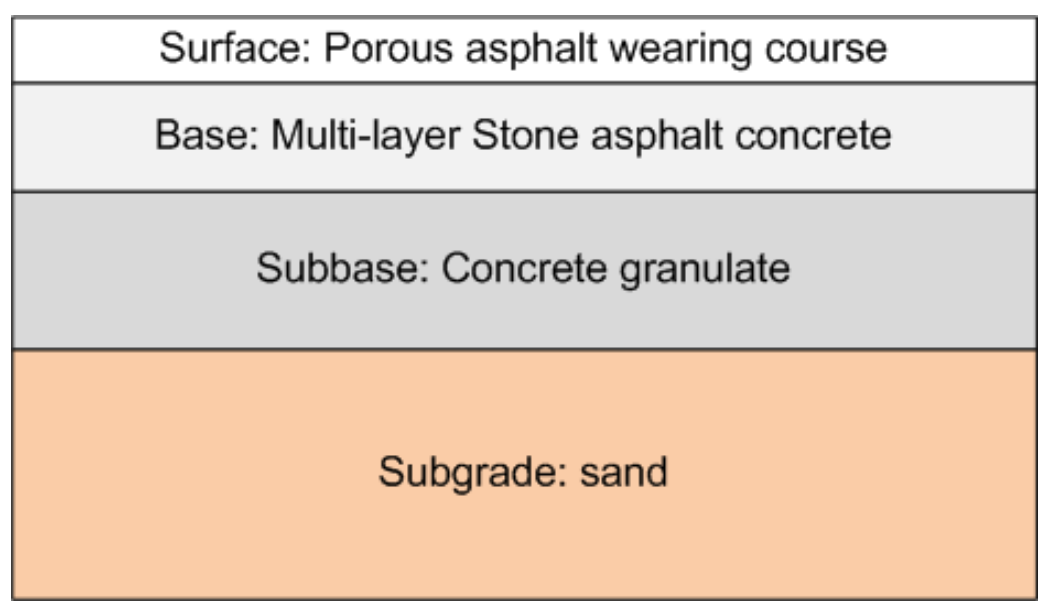

Figure 2.17 Structure of a typical asphalt pavement. 
Table 2-1 Properties of Asphalt concrete

\begin{tabular}{|l|l|c|c|}
\hline \multicolumn{2}{|c|}{ Material } & $\begin{array}{c}\text { Young's Elastic Modulus E } \\
(\mathrm{GPa})\end{array}$ & Poisson's Ratio $\mu$ \\
\hline $\begin{array}{l}\text { Asphalt } \\
\text { concrete } \\
{[35]}\end{array}$ & $0^{\circ} \mathrm{C}$ & $13.5-35$ & $0.25-0.30$ \\
\cline { 2 - 4 } & $20^{\circ} \mathrm{C}$ & $2-3.5$ & $0.30-0.35$ \\
\cline { 2 - 4 } & $60^{\circ} \mathrm{C}$ & $0.15-0.35$ & $0.35-0.40$ \\
\hline
\end{tabular}

\subsubsection{KEHs comparison for roadway energy harvesting}

The key parameters of dielectric elastomer KEHT, electromagnetic KEHT and piezoelectric materials are presented in Table 2-2. It shows that dielectric elastomer KEHT has the largest theoretical maximum energy density due to its large maximum strain and flexibility, but the need of a poling voltage in the first stage of its working cycles makes the dielectric elastomer KEHT difficult to implement. Dielectric elastomer KEHTs usually need an external battery or a solar panel to supply the electric charges for the cold start. In addition, compared with the asphalt pavement, dielectric elastomer KEHT's Young's modulus is much lower; therefore it is more suited for harvesting energy from energy sources where large motions are allowed.

Electromagnetic KEHT has moderate theoretical maximum energy density and developed technologies; however, for KEHT on roads, it needs a complicated mechanical coupling (hydraulic system or gear box) to convert the low frequency force impact from vehicles to the rotation motions of the rotors. Therefore, the installation and maintenance is difficult, and the failure modes are increased.

Piezoelectric materials are of very high stiffness which is close to that of typical asphalt concrete, so piezoelectric transducers installed in asphalt pavements only have limited 
effects on the driving experience. Another advantage is that the lifetime of PZT materials is almost infinite if the applied force and the temperature are in the operational range [36], therefore the maintenance is eased. These advantages make piezoelectric materials suitable for roadway energy harvesting. Among the present two piezoelectric materials: PZT and PVDF, PZT is the hardest and has the largest theoretical maximum energy density (hundred times more than the PVDF) according to Table 2-2. Therefore, compared to dielectric elastomer and electromagnetic generator, the piezoelectric material PZT is more competitive in the design of SRPHS.

Table 2-2 Comparison of different KEHTs

\begin{tabular}{|l|l|l|l|l|}
\hline \multicolumn{2}{|l|}{ Material } & $\begin{array}{l}\text { Typical } \\
\text { Young's } \\
\text { modulus } \\
(\mathrm{GPa})\end{array}$ & $\begin{array}{l}\text { Maximum } \\
\text { Strain(\%) }\end{array}$ & $\begin{array}{l}\text { Theoretical } \\
\text { Maximum } \\
\text { energy } \\
\text { density }\left(\mathrm{mJ} / \mathrm{cm}^{3}\right)\end{array}$ \\
\hline $\begin{array}{l}\text { Dielectric } \\
\text { elastomer }\end{array}$ & Silicone [37] & $1 \times 10^{-3}$ & 100 & 750 \\
\cline { 2 - 5 } & Acrylic [38] & $2 \times 10^{-3}$ & 380 & 3400 \\
\hline Electromagnetic generator [39] & NA & NA & 400 \\
\hline Piezoelectric & $\begin{array}{l}\text { Ceramic(PZT) } \\
{[40]}\end{array}$ & $50-100$ & $0.15-0.20$ & $50-90$ \\
\cline { 2 - 5 } & $\begin{array}{l}\text { Polymer(PVDF) } \\
{[41]}\end{array}$ & $2-4$ & 0.1 & $0.02-0.04$ \\
\hline
\end{tabular}




\section{Chapter: PZT Energy Transducer}

\subsection{Background of piezoelectricity}

Prior to the selection of the proper PZT materials and the design of the PZT transducer, the basic concepts, equations, and coefficients of piezoelectricity are worthwhile to be reviewed.

\subsubsection{Direct piezoelectric effect and converse piezoelectric effect}

The direct piezoelectric effect, by which certain materials can be electrically polarized when subjected to a mechanical force, was first experimentally demonstrated by the brothers Pierre Curie and Jacques Curie using a series of crystals like quartz, tourmaline, topaz, cane sugar and Rochelle salt in 1880 [42]. These crystals generated charges are proportional but opposite to the applied forces. In 1881, the next year, Gabriel Lippmann mathematically deduced the opposite effect, which states that certain crystals will experience a mechanical strain when subjected to an electric field. This effect called converse piezoelectric effect was then experimentally confirmed by the Curies brother soon after.

Although the maximum strain of piezoelectric materials is very small $(0.15 \%$ to $0.2 \%)$ [40], a great range of applications have been discovered in industries. For example, the piezoelectric effect is used in high-accuracy sensors such as force sensors and displacement sensors, and the converse piezoelectric effect is used in actuators such as sonar, vibrators or precise position control devices. For many years crystalline minerals 
like quartz or Rochelle salt are the main source of piezoelectric materials. It was not until 1954 that man-made piezoelectric materials, the PZT ceramics, were discovered by Jaffe. Typically PZT has the chemical formula $\mathrm{Pb}\left[\mathrm{Zr}_{\mathrm{x}} \mathrm{Ti}_{1-x}\right] \mathrm{O}_{3}$, which has a perovskite crystal structure with an electric dipole if the crystal is below its Curie temperature point (shown in Figure 3.1).

Before polarization, dipole moments of crystals in different regions of the ceramic are oriented randomly and the ceramic is isotropic, and therefore the overall dipole moments of the ceramic are equal to zero. The polarization (or poling) process is performed by exposing the ceramic to a strong electric field for a certain period of time, below the Curie point of temperature. After removing the electric field, the ceramic will hold a permanent residual polarization and lose its isotropic nature.

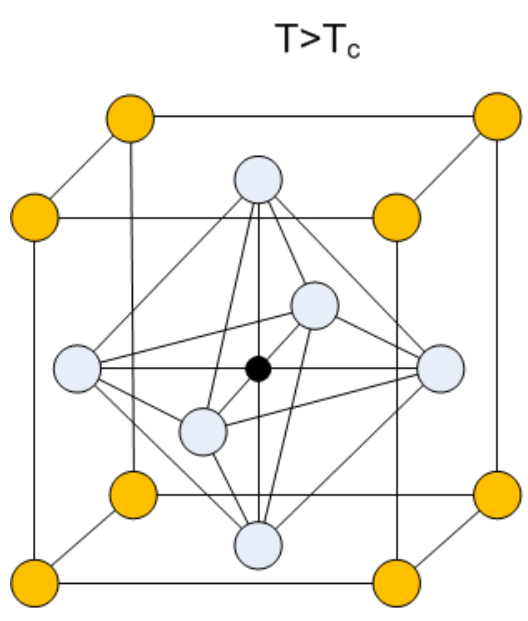

(a)

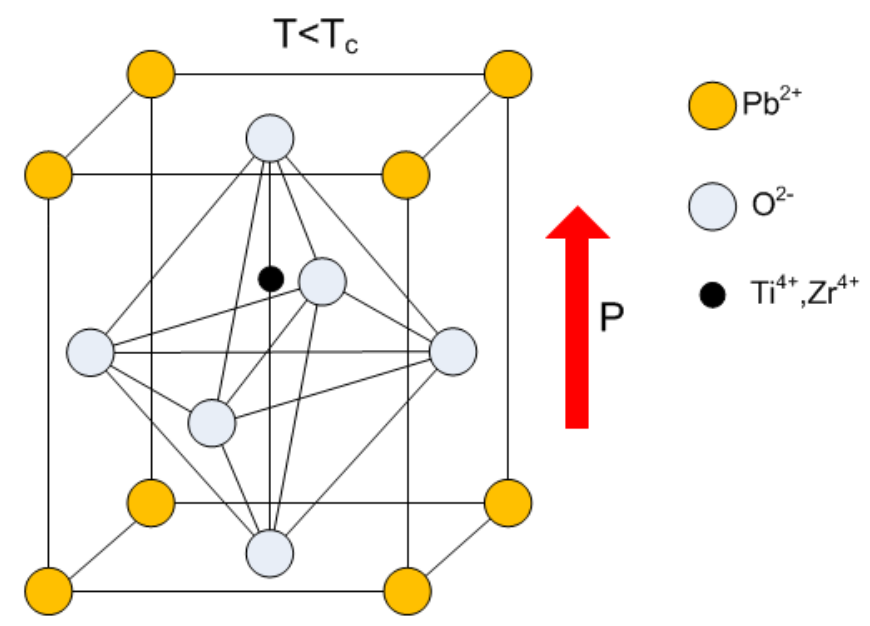

(b)

Figure 3.1 PZT perovskite crystal structure: (a) above Curie point, balanced positive and negative charges; (b) below Curie point, crystal has electric dipole moment [43]. 
Under mechanical stress, positive irons and negative irons in the ceramic crystal structure experience relative movement. The deformation caused by stress changes the overall dipole moment of the ceramic and causes a potential difference between the electrodes of the ceramic in an open circuit condition. The polarity of the open circuit voltage is determined by the polarization and stress direction together as indicated in Figure 3.2. The PZT ceramic that deforms to be thinner and broader either by compression along the poling direction or tension perpendicular to the poling direction generates voltage of the same direction as the poling (Figure 3.2(a)). The PZT ceramic that deforms to be taller and slimmer either by tension along poling direction or compression perpendicular to poling direction will generate voltage of the opposite direction to the poling (Figure 3.2(a)). This process is called "generator action" corresponding to the direct piezoelectric effect that converts mechanical energy to electric energy.
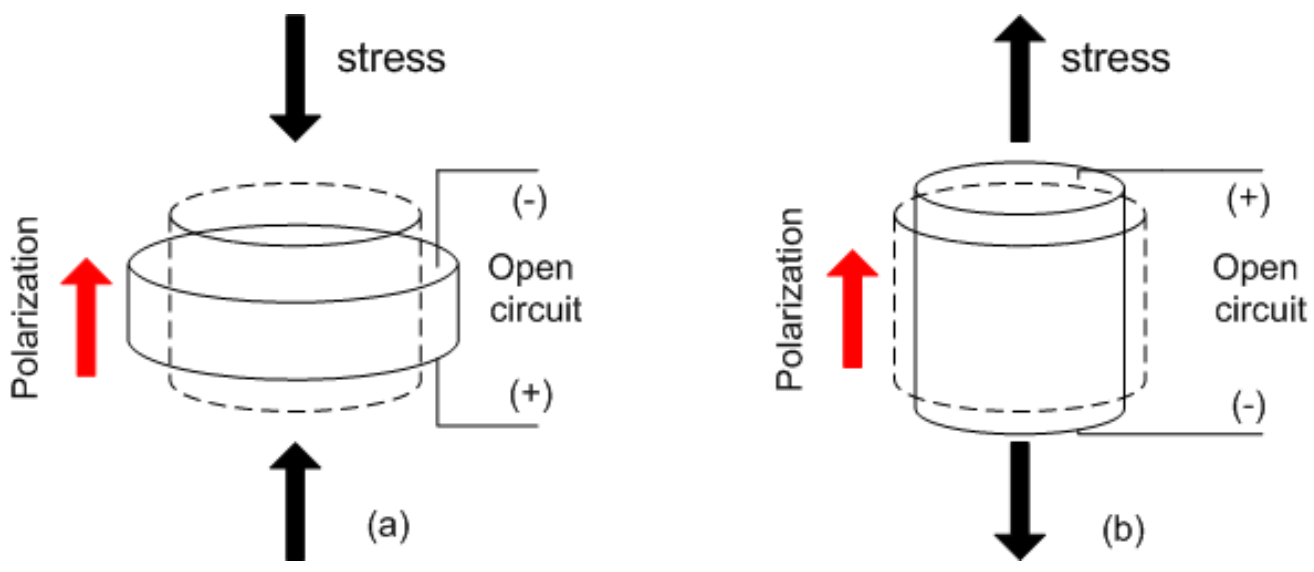

Figure 3.2 Direct piezoelectric effect: (a) under compression; (b) under tension (dashed shape indicates initial state). 
On the other hand, under an applied voltage across the PZT ceramic's electrodes, the ceramic deforms accordingly. The ceramic deformation depends on the poling field direction and the applied voltage direction as indicated in Figure 3.2. Voltage applied along the poling direction deforms the ceramic to be thicker and slimmer (Figure 3.3(a)), and voltage applied opposite to the poling direction deforms the ceramic to be thinner and broader (Figure 3.3(b)). This process is called "motor action" corresponding to the converse piezoelectric effect that converts electric energy to mechanical energy.

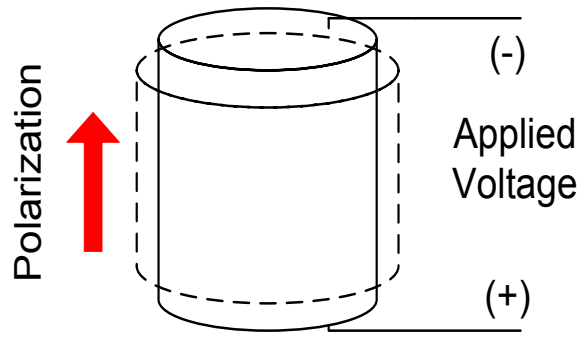

(a)

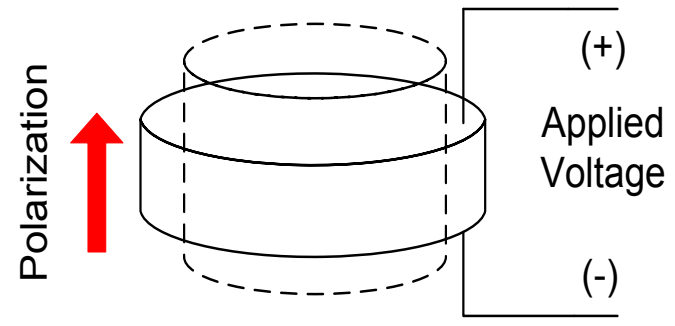

(b)

Figure 3.3 Converse piezoelectric effect: (a) under applied voltage same to the polarization direction; (b) under applied voltage opposite to the polarization direction (dashed shape indicates initial state).

\subsubsection{Piezoelectric governing equations}

PZT ceramic is an anisotropic material, which means its physical properties (piezoelectric constant, elastic compliance, permittivity) are all tensors related to the mechanical forces and electric fields in different directions. The linear constitutive tensor equations of piezoelectricity (in strain-charge form) are as follows [44]:

$$
\left\{\begin{array}{l}
S \\
D
\end{array}\right\}=\left[\begin{array}{cc}
S^{E} & d^{\prime} \\
d & \varepsilon^{T}
\end{array}\right]\left\{\begin{array}{l}
T \\
E
\end{array}\right\}
$$


In Equation (3.1), $T$ represents stress and is a second-rank tensor:

$$
T=\left[\begin{array}{ccc}
T_{11} & T_{12} & T_{13} \\
\vdots & T_{22} & T_{23} \\
\text { sym } & \ldots & T_{33}
\end{array}\right]
$$

$T$ has six independent values and can be written in the Voigt form:

$$
T=\left[\begin{array}{l}
T_{11} \\
T_{22} \\
T_{33} \\
T_{23} \\
T_{13} \\
T_{12}
\end{array}\right]=\left[\begin{array}{l}
T_{1} \\
T_{2} \\
T_{3} \\
T_{4} \\
T_{5} \\
T_{6}
\end{array}\right]
$$

$S$ represents strain and is a second-rank tensor:

$$
S=\left[\begin{array}{ccc}
S_{11} & S_{12} & S_{13} \\
\vdots & S_{22} & T_{23} \\
s y m & \cdots & S_{33}
\end{array}\right]
$$

In the Voigt form,

$$
S=\left[\begin{array}{l}
S_{11} \\
S_{22} \\
S_{33} \\
S_{23} \\
S_{13} \\
S_{12}
\end{array}\right]=\left[\begin{array}{l}
S_{1} \\
S_{2} \\
S_{3} \\
S_{4} \\
S_{5} \\
S_{6}
\end{array}\right]
$$

$D$ represents the electric displacement and is a three component vector:

$$
D=\left[\begin{array}{l}
D_{1} \\
D_{2} \\
D_{3}
\end{array}\right]
$$

$E$ represents the electric field vector with three components:

$$
E=\left[\begin{array}{l}
E_{1} \\
E_{2} \\
E_{3}
\end{array}\right]
$$


For the PZT ceramics physical properties matrices, $\left[s^{\mathrm{E}}\right]$ is the elastic compliance (strain generated per unit stress) under a constant electric field $E$, and it is a fourth-rank tensor represented as a $6 \times 6$ matrix.

$$
s^{\mathrm{E}}=\left[\begin{array}{llllll}
s_{11} & s_{12} & s_{13} & s_{14} & s_{15} & s_{16} \\
s_{21} & s_{22} & s_{23} & s_{24} & s_{25} & s_{26} \\
s_{31} & s_{32} & s_{33} & s_{34} & s_{35} & s_{36} \\
s_{41} & s_{42} & s_{43} & s_{44} & s_{45} & s_{46} \\
s_{51} & s_{52} & s_{53} & s_{54} & s_{55} & s_{56} \\
s_{61} & s_{62} & s_{63} & s_{64} & s_{65} & s_{66}
\end{array}\right]
$$

$d$ is the piezoelectric charge constant, and it is a third-rank tensor that can be represented as a $3 \times 6$ matrix:

$$
d=\left[\begin{array}{llllll}
d_{11} & d_{12} & d_{13} & d_{14} & d_{15} & d_{16} \\
d_{21} & d_{22} & d_{23} & d_{24} & d_{25} & d_{26} \\
d_{31} & d_{32} & d_{33} & d_{34} & d_{35} & d_{36}
\end{array}\right]
$$

$\varepsilon^{T}$ is the absolute permittivity (dielectric displacement per unit electric field) matrix under constant stress $T$, and it is a second-rank tensor:

$$
\varepsilon^{T}=\left[\begin{array}{lll}
\varepsilon_{11} & \varepsilon_{12} & \varepsilon_{13} \\
\varepsilon_{21} & \varepsilon_{22} & \varepsilon_{23} \\
\varepsilon_{32} & \varepsilon_{32} & \varepsilon_{33}
\end{array}\right]
$$

Equation (3.1) can also be written as:

$$
\{S\}=\left[s^{\mathrm{E}}\right]\{T\}+\left[\mathrm{d}^{\prime}\right]\{E\}
$$




$$
\{D\}=[d]\{T\}+\left[\varepsilon^{T}\right]\{E\}
$$

Equation (3.11) is the governing equation for the converse piezoelectric effect under

constant electric field. The first term $\left[s^{\mathrm{E}}\right]\{T\}$ represents the strain caused by the external stress, and the second term $\left[\mathrm{d}^{\prime}\right]\{E\}$ represents the strain produced by the external electric field. Equation (3.12) is the governing equation for the direct piezoelectric effect under constant stress condition (assuming the PZT ceramics are able to change dimensions freely with the applied force). The first term $[d]\{T\}$ is the electric displacement caused by the external stress, and the second term $\left[\varepsilon^{T}\right]\{E\}$ is the electric displacement produced by the external electric field.

\subsubsection{Piezoelectric coefficients in different modes of direct piezoelectric effect}

The possible stress directions of a piezoelectric element in a right-handed Cartesian coordinate system are shown in Figure 3.4, where x, y, z axes are represented by 1, 2, 3 . Usually PZT ceramics used in sensors and actuators are polarized in the direction 3, and therefore they are cylindrically symmetric about that direction. Accordingly, the independent parameters in piezoelectric coefficient matrices $[d]$ can be reduced to three according to the three modes: 33-mode (longitudinal mode), 31-mode (transverse mode), and 15-mode (shear mode). Because the piezoelectric material has a crystalline structure poled in a way that makes it cylindrically symmetric about its poled axis. The piezoelectric charge coefficient matrix can be reduces:

$$
[d]=\left[\begin{array}{cccccc}
0 & 0 & 0 & 0 & d_{15} & 0 \\
0 & 0 & 0 & d_{15} & 0 & 0 \\
d_{31} & d_{31} & d_{33} & 0 & 0 & 0
\end{array}\right]
$$




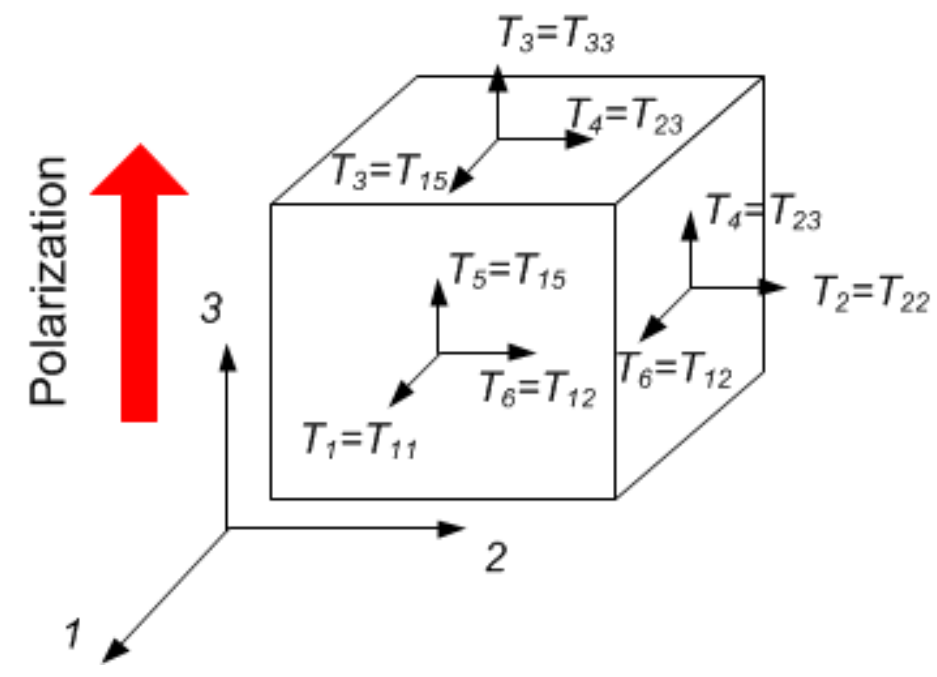

Figure 3.4 Possible stress directions of a piezoelectric element.

The three modes for direct piezoelectric effect (or generator action) are shown in Figure 3.5. The PZT stacks in the figure are all polarized in direction 3. In 33-mode, the stack is stretched in direction 3, and electric charges are generated on face 3. In 31-mode, the stack is stretched in direction 1, and charges are produced on face 3 as well. In 15-mode, shear stress is applied on face 1 in direction 3 , and charges are generated on face 1.

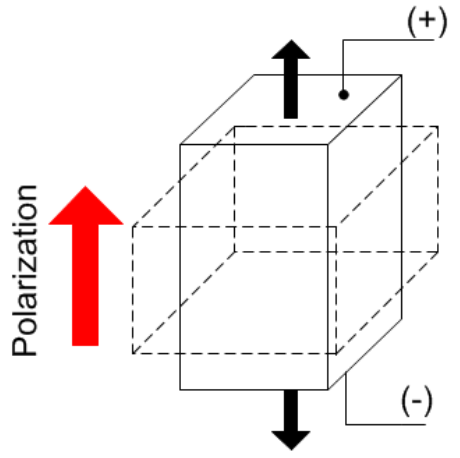

(a)

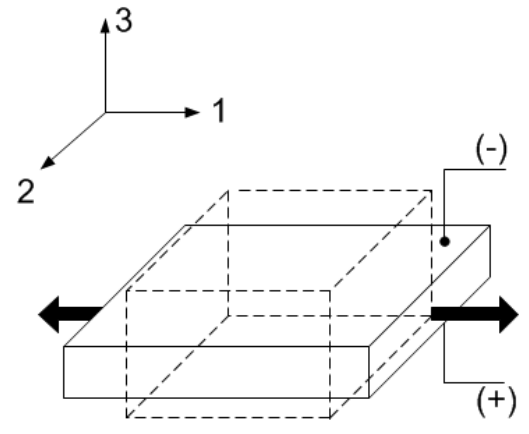

(b)

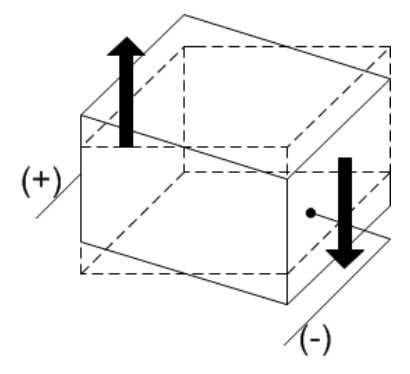

(c)

Figure 3.5 Three direct piezoelectric modes: (a) 33-mode; (b) 31-mode; (c) 15-mode. 
For PZT ceramics working in direct piezoelectric effect under constant external electric field $E=0$, Equation (3.12) can be simplified to:

$$
\{D\}=[d]\{T\}
$$

Combining (3.14) with (3.13),

$$
\begin{gathered}
\left\{\begin{array}{c}
D_{1} \\
D_{2} \\
D_{3}
\end{array}\right\}=\left[\begin{array}{cccccc}
0 & 0 & 0 & 0 & d_{15} & 0 \\
0 & 0 & 0 & d_{15} & 0 & 0 \\
d_{31} & d_{31} & d_{33} & 0 & 0 & 0
\end{array}\right]\left\{\begin{array}{l}
T_{1} \\
T_{2} \\
T_{3} \\
T_{4} \\
T_{5} \\
T_{6}
\end{array}\right\} \\
=\left\{\begin{array}{c}
d_{15} T_{5} \\
d_{15} T_{4} \\
d_{31} T_{1}+d_{31} T_{2}+d_{33} T_{3}
\end{array}\right\}
\end{gathered}
$$

For the three modes of direct piezoelectric effect, the electric displacement in each mode shown in Figure 3.5 can be derived from Equation (3.15):

$$
\begin{cases}D_{3}=d_{33} T_{3} & 33 \text { mode } \\ D_{3}=d_{31} T_{2} & 31 \text { mode } \\ D_{2}=d_{15} T_{4} & 15 \text { mode }\end{cases}
$$

The electric displacement $D$ developed in the PZT ceramic is related to the electric field $E$ as:

$$
D=\varepsilon \cdot E
$$

where $[\varepsilon]$ is the absolute permittivity matrix of the dielectric material.

Combining Equation (3.14) and (3.17): 


$$
\begin{aligned}
E & =\frac{D}{\varepsilon}=\frac{d}{\varepsilon} \cdot T \\
& =g \cdot T
\end{aligned}
$$

where $g=\frac{d}{\varepsilon}$ is defined as the piezoelectric voltage coefficient. For the three modes of direct piezoelectric effect, Equation (3.18) becomes:

$$
\begin{cases}E_{3}=g_{33} T_{3} & 33 \text { mode } \\ E_{3}=g_{31} T_{2} & 31 \text { mode } \\ E_{2}=g_{15} T_{4} & 15 \text { mode }\end{cases}
$$

Another important coefficient of piezoelectric materials is the piezoelectric electromechanical coupling coefficient $k$, defined as the efficiency of the conversion between mechanical energy and electric energy:

$$
k^{2}=\frac{\text { converted energy }}{\text { input energy }}
$$

\begin{tabular}{|c|c|c|c|c|}
\hline \multicolumn{2}{|c|}{$\begin{array}{c}\text { Piezoelectric } \\
\text { Coefficient }\end{array}$} & \multicolumn{2}{|c|}{ Definition } & Unit \\
\hline \multirow{3}{*}{$\begin{array}{l}\text { Piezoelectri } \\
\text { c charge } \\
\text { constant } \\
\left(d_{i j}\right)\end{array}$} & $d_{33}$ & $\begin{array}{c}\text { Electrical } \\
\text { polarization }\end{array}$ & $\frac{D_{3}}{T_{3}}$ & \multirow{3}{*}{$C / N$} \\
\hline & $d_{31}$ & $\begin{array}{l}\text { in direction } i \\
\text { per unit } \\
\text { mechanical }\end{array}$ & $\frac{D_{3}}{T_{1}}$ & \\
\hline & $d_{15}$ & $\begin{array}{c}\text { stress in } \\
\text { direction } j\end{array}$ & $\frac{D_{1}}{T_{5}}$ & \\
\hline $\begin{array}{c}\text { Piezoelectri } \\
\text { c voltage }\end{array}$ & $g_{33}$ & $\begin{array}{l}\text { Electric field } \\
\text { in direction } i\end{array}$ & $\frac{E_{3}}{T_{3}}$ & $V \cdot m / N$ \\
\hline
\end{tabular}

Table 3-1 summarizes the definitions and the units of piezoelectric coefficients of the three modes.

Table 3-1 Piezoelectric coefficients of three modes 


\begin{tabular}{|c|c|c|c|c|}
\hline \multirow[t]{2}{*}{$\begin{array}{l}\text { constant } \\
\quad\left(g_{i j}\right)\end{array}$} & $g_{31}$ & \multirow{2}{*}{$\begin{array}{c}\text { per unit } \\
\text { mechanical } \\
\text { stress in } \\
\text { direction } j\end{array}$} & $\frac{E_{3}}{T_{1}}$ & \\
\hline & $g_{15}$ & & $\frac{E_{1}}{T_{5}}$ & \\
\hline \multirow{5}{*}{$\begin{array}{l}\text { Piezoelectri } \\
\text { c coupling } \\
\text { factor } k\end{array}$} & $k_{33}$ & \multirow{5}{*}{$\begin{array}{l}\text { Factor of } \\
\text { energy } \\
\text { conversion } \\
\text { efficiency }\end{array}$} & ceramic rod with $E_{3}$ and $T_{3}$ & \multirow{5}{*}{$N A$} \\
\hline & $k_{31}$ & & ceramic rod with $E_{3}$ and $T_{1}$ & \\
\hline & $k_{15}$ & & ceramic rod with $E_{1}$ and $T_{5}$ & \\
\hline & $k_{t}$ & & ceramic thin disk with $E_{3}$ and $T_{3}$ & \\
\hline & $k_{p}$ & & ceramic thin disk with $E_{3}$ and $T$ in radius & \\
\hline
\end{tabular}

\subsection{PZT transducer}

Presently, there are a couple of PZT transducer designs available and each of them is suitable for a particular mechanical energy source. Thus, the characteristics of the loading of vehicles are necessary to be analyzed first.

\subsubsection{Kinetic energy source of asphalt pavement}

As a wheel of a vehicle rolls over the pavement, the wheel deforms and has a contact area with the pavement surface, as shown in Figure 3.6. It is assumed that the vehicle is a typical four-wheel family car, with 1.5 tons of total weight $(W)$ and tire pressure $\left(P_{t}\right)$ between 0.2 to $0.25 \mathrm{MPa}$. On the contact area, the pressure on the pavement is the same with the tire pressure. It should be noted that the stress in the pavement is spread out and reduced in intensity [33] as shown by the dash line in Figure 3.6, and this means that the PZT transducer should be installed as close as possible to the surface [34]. The loading on each wheel $W$ is $\frac{M \cdot g}{4}=3675 N$, and the wheel-pavement contact area $A$ is assumed to be rectangular [45] and is, therefore, between $140 \mathrm{~cm}^{2}$ and $180 \mathrm{~cm}^{2}$ depending on the tire pressure (0.2-0.25 MPa). 


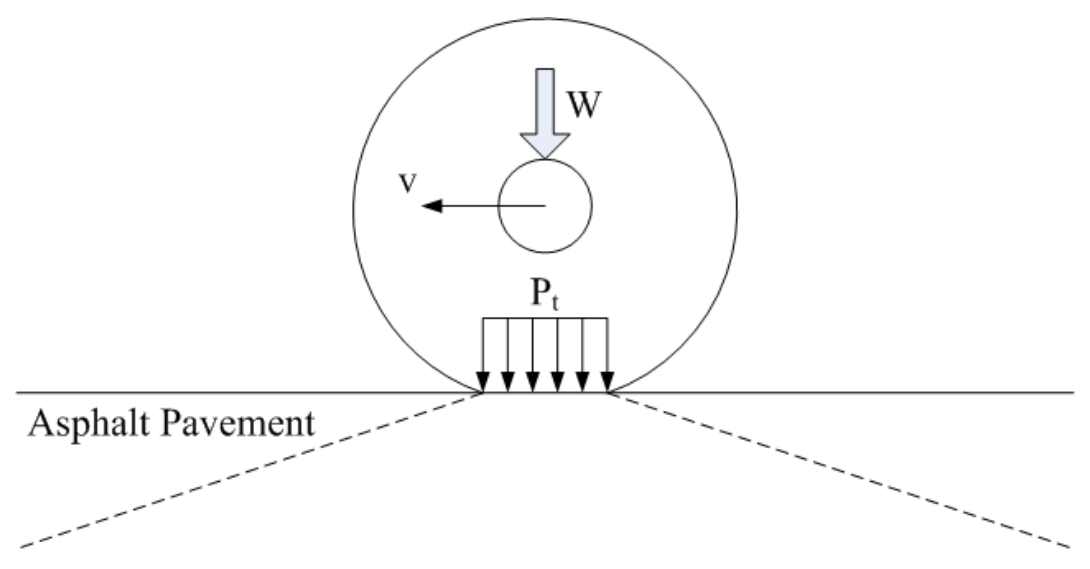

Figure 3.6 The wheel load of pavement.

The frequency of the wheel loading depends on the vehicle's velocity $v$, the wheel base (more specifically, the distance between the front wheel center and the back wheel center) of the vehicle, and the distance between the vehicles. For example, assuming a common family car with the wheel base of $2.9 \mathrm{~m}$, and a series of such cars is running at a low speed, $30 \mathrm{~km} / \mathrm{h}$, on busy commercial areas with a safe following distance of two seconds' driving [46]. Then the time interval between the front wheel and the back wheel of each vehicle is about $0.35 \mathrm{~s}$, and the time interval between cars is assumed to be $2 \mathrm{~s}$.

The kinetic energy source in asphalt pavement with light vehicles can therefore be modeled as a series of force impacts with the following parameters: compression stress $(0.2-0.25 \mathrm{MPa})$, contact area $\left(140 \mathrm{~cm}^{3}-180 \mathrm{~cm}^{3}\right)$, and low frequency $(0.5$ to $3 \mathrm{HZ})$.

\subsubsection{Overview of PZT transducers}

Though it is tempting and easier to use PZT ceramic plates or disks directly in 33-mode to harvest kinetic energy on roads, as has been done by Innowattech [26]. The generated 
energy is limited due to the high stiffness of the PZT ceramics. For this reason, PZT transducers with certain structural configurations are required to lower its overall stiffness and amplify the internal stress in PZT. Figure 3.7 lists popular PZT transducers developed in recent years.

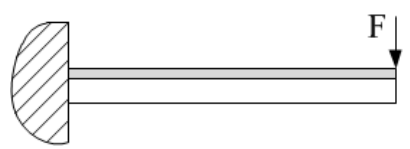

(a)

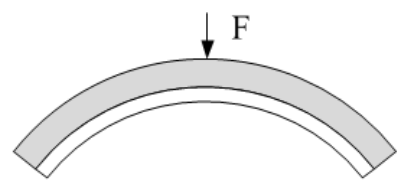

(c)

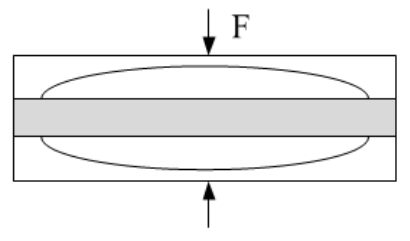

(e)

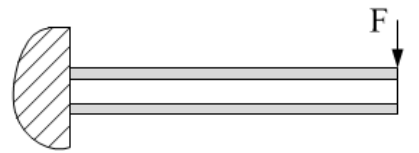

(b)

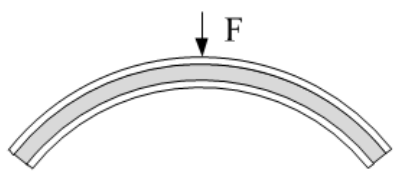

(d)

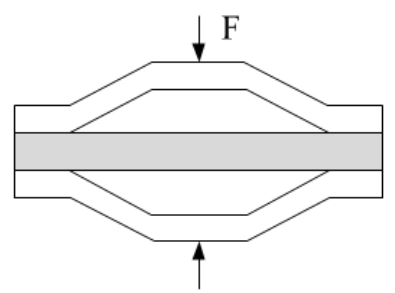

(f)
Metal layer

PZT layer

Figure 3.7 Typical configurations of PZT transducers: (a) Unimorph; (b) Bimorph; (c) RAINBOW; (d) THUNDER; (e) Moonie; (f) Cymbal.

Piezoelectric Unimorph transducers (Figure 3.7(a)) are composed of a strip of PZT and a strip of metal bonded together, and Bimorph transducers (Figure 3.7(b)) are composed of two strips of PZT bonded on both sides of the metal strip [47]. Piezoelectric Unimorph and Biomorph transducers have similar structures. When they are bent, electric charges are generated between the electrodes. They are usually constructed as cantilevers with a proof load at the free end to adjust its natural frequency and harvest kinetic energy from vibrational sources. 
RAINBOW (Reduced and Internally Biased Oxide Wafer) transducers (Figure 3.7(c)) were developed by Haertling in 1994 [48]. Their structure is similar to the Unimorph, but it is bent beforehand. They have a monolithic dome structure which is composed of a top layer of PZT and an oxygen reduced layer in the bottom. The oxygen reduced layer is formed by placing one side of the PZT wafer in contact with a carbon block with high temperature. As the carbon is oxidized, the PZT on that side is transformed into an electrode layer of lead. Under downward compression, the PZT layer in RAINBOW transducers experiences transverse stress and generates voltage. However, as both the PZT layer and the reduced layer are brittle, RAINBOW transducers cannot endure the high load from the vehicle.

THUNDER (Thin-layer composite unimorph ferroelectric driver and sensor) transducers (Figure 3.7(d)) were developed by NASA and Face International corporation in 1997 [49]. They are composed of a PZT wafer laminated between two metal backings (aluminum and stainless steel). Both RAINBOW and THUNDER transducers are categorized as "unimorph prestressed bending transducers". They have a shape of arc that elongates under a force applied to the arc top. Compared with RAINBOW transducers, THUNDER transducers are more flexible, but its stiffness is still far less than that of the pavement [50].

Moonie transducers (Figure 3.7(e)) were designed in the Pennsylvania State University in 1993 [51]. They consist of a PZT ceramic disk sandwiched between two metal end caps. 
The end caps are essentially mechanical transformers that can transform the axial deformation of the endcaps into the radial deformation of the PZT ceramic disk. Cymbal transducers (Figure 3.7(f)) were designed and improved based on the Moonie transducer in the Pennsylvania State University in 1997 [52]. Both Moonie and Cymbal belong to the category of flextensional transducers. Their stiffness fulfills the stiffness gap between the PZT stacks and the unimorph or bimorph transducers, and they are able to be designed compatible with the pavement.

Compared with Moonie transducers, Cymbal transducers have larger contact surface, higher stability under loading, and lower cost due to the simplicity in Cymbal endcaps' fabrication [52] [53]. Accordingly, the PZT Cymbal transducer is chosen to be the kinetic energy harvester used on asphalt pavement. The Cymbal transducer needs to be modified and optimized to meet the requirements of harvesting kinetic energy due to the high load of vehicles.

\subsection{Cymbal Energy Transducer Design}

The following sections provide the design procedure of the Cymbal transducer. Its geometry is improved and optimized to harvest the most energy from the load of vehicle wheels. Firstly a simple Cymbal model is built to determine the crucial piezoelectric properties that affect the generated energy. Then a Finite Element Analysis (FEA) is implemented so as to choose the optimal structural parameters combination. Kim et al. 
have built a FEA model of the Cymbal transducer [54], but only the thickness of the metal endcaps and the PZT ceramics are considered as variables. Zhao et al. have evaluated the Cymbal FEA model on all its dimensions [24], but the Cymbal model is assumed to be surrounded by asphalt concrete directly (Model A in Figure 2.11). The Cymbal FEA model built in this section considers all the dimensions, and is assumed to have a contact load just on the apex of the endcaps.

\subsubsection{Structural parameters of Cymbal Transducer}

The Cymbal transducer is composed of two identical concave-shaped metal endcaps and one PZT ceramic disk. The metal endcaps are bonded on two sides of the ceramic disk and are used as electrodes as well. As shown in Figure 3.8, the diameter of the endcaps are the same as the PZT disk diameter $D$, and the height $d_{c}$ and the base diameter $D_{c}$ of the air cavity are two parameters that determines the effective piezoelectric charge coefficient $d_{e f f}$ of the Cymbal transducer [55]. $D_{d}$ is the apex diameter of the endcaps, and $t_{m}$ and $t_{p}$ are the thickness of the endcaps and the PZT disks respectively.

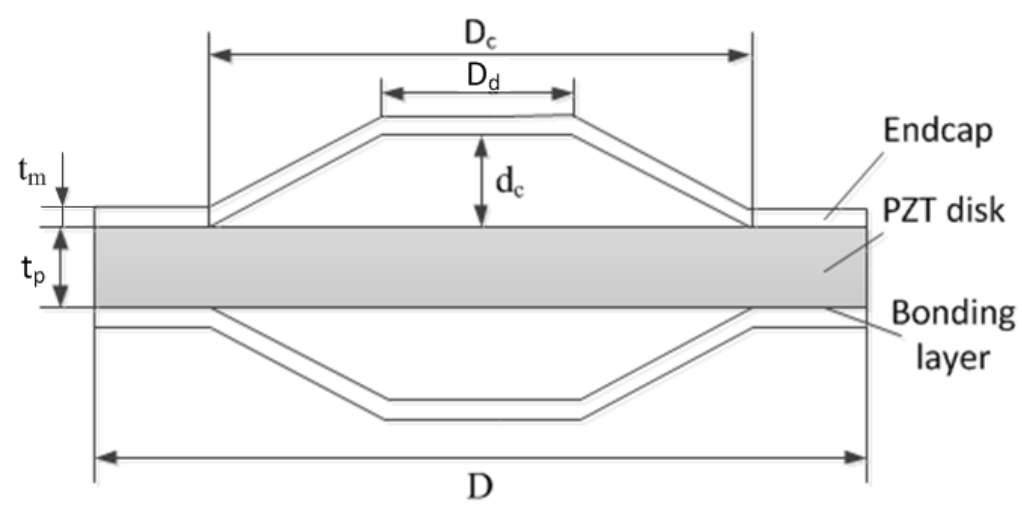

Figure 3.8 Cymbal Transducer configuration. 
The structural dimensions and materials of the Cymbal have crucial effects on its performance such as the generated power, stiffness, and maximum permissive load. A FEA model of Cymbal transducer is analyzed in the following sections so as to provide guidance for the selection of the dimensions and materials of the Cymbal transducer.

\subsubsection{Analysis of Cymbal model under compression}

The endcap of the Cymbal transducer can be modeled as a truncated conical shell [56]. The Cymbal transducer is vertically symmetrical, and the upper part of Cymbal is shown in Figure 3.9.

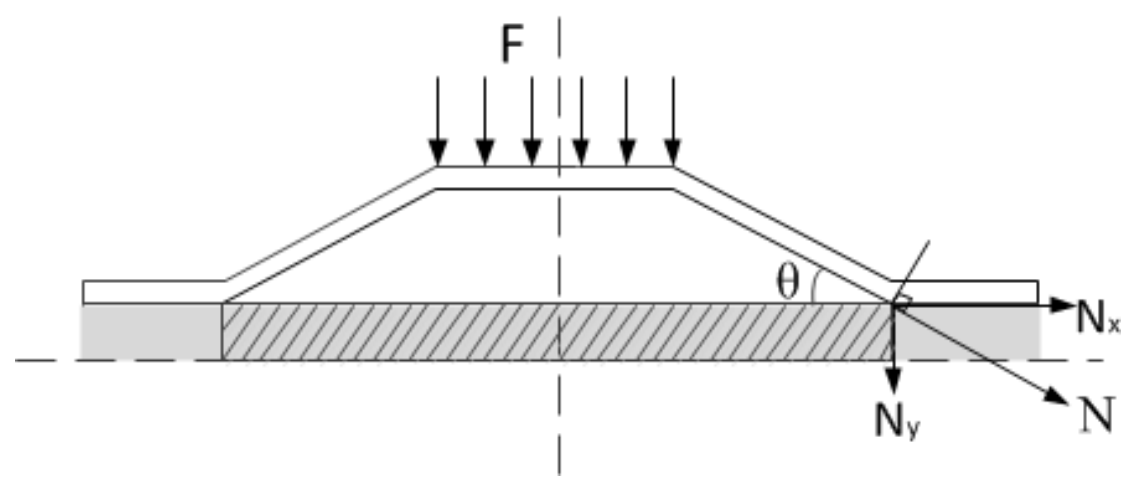

Figure 3.9 Endcaps under load.

According to Figure 3.9, if the endcap's apex has an axial load $F$, the pressure from the endcap to the air cavity base rim will be:

$$
N=\frac{F}{\pi D_{c} t_{m} \sin \theta}
$$

The pressure $N$ can be decomposed into two directions: axial $N_{y}$ and radial $N_{x}$. Then the stresses of the shell in these two directions are: 


$$
\left\{\begin{array}{l}
T_{x}=N \cos \theta=\frac{F \cos \theta}{\pi t_{m} D_{c} \sin \theta} \\
T_{y}=-N \sin \theta=-\frac{F}{\pi t_{m} D_{c}}
\end{array}\right.
$$

Considering the endcap on the other side, the cavity region (shadow part in Figure 3.9) of the PZT disk is subjected to a radial tensile stress $T_{r}$, while the bonding region of the PZT disk is subjected to a compressive stress $T_{b}$.

$$
\left\{\begin{array}{l}
T_{r}=\frac{2 N_{x}}{t_{p}}=\frac{2 F}{\pi t_{p} D_{c} \tan \theta} \\
T_{b}=\frac{4 F}{\pi\left(D^{2}-D_{c}^{2}\right)}
\end{array}\right.
$$

Equation (3.23) shows the radial tensile stress $T_{r}$ is inversely proportional to $t_{p}, D_{c}$ and $\theta$ under a constant force $F$.

\subsubsection{Selection of PZT ceramic material}

It is reasonable to assume that the tensile stress is concentrated in the cavity region of the PZT disk, while the compression stress is mainly concentrated in the bonding region of the disk. Therefore, the PZT disk in the Cymbal transducer works in both 31 mode by tensile stress and 33 mode by compressive stress. Assuming a PZT disk with a radius of $r$ and a thickness of $h$ is subjected to an average cyclic radial tensile stress $T_{r}$ and an average compressive stress $T_{c}$ as shown in Figure 3.10. The generated electric field $E_{3}$ can be derived from Equation (3.19):

$$
E_{3}=g_{31} T_{r}+g_{33} T_{c}
$$




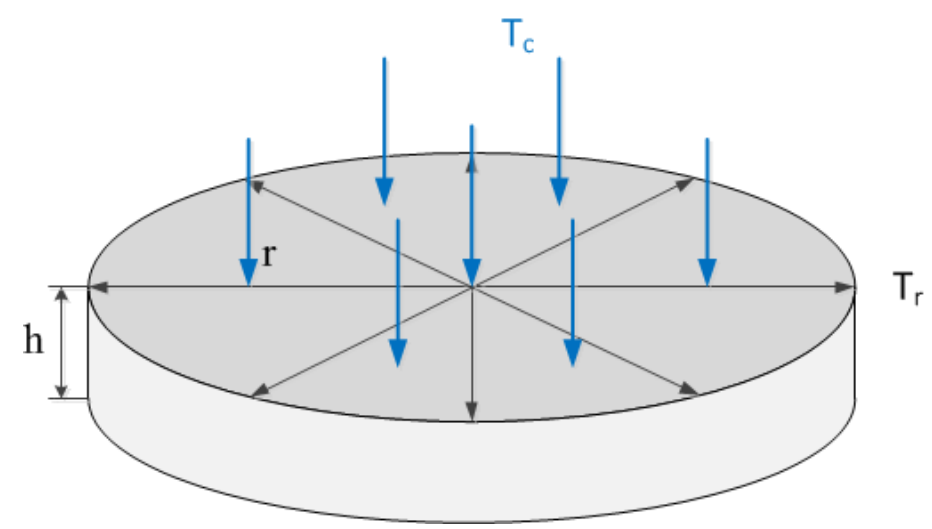

Figure 3.10 PZT disk under radial tensile stress and compressive stress.

Then the open circuit voltage $V$ of the PZT disk is the multiplication of the electric field $E$ between electrodes and their interval $h$,

$$
\begin{aligned}
V & =E_{3} h \\
& =\left(g_{31} T_{r}+g_{33} T_{c}\right) h
\end{aligned}
$$

Assuming that $T_{c}=A T_{r}$, where $A$ is the ratio between $T_{c}$ and $T_{r}$, Equation (3.25) becomes:

$$
\begin{aligned}
V & =\left(g_{31}+A g_{33}\right) T_{r} h \\
& =g_{e f f} T_{r} h
\end{aligned}
$$

where $g_{\text {eff }}$ represents the effective piezoelectric voltage coefficient and is contributed by both $g_{31}$ and $g_{33}[57,58]$.

As the PZT disk can be seen as a capacitor $C$ in parallel with a current source $[59,60]$, the generated electric energy under stress can be calculated: 


$$
\begin{gathered}
W=\frac{1}{2} C V^{2}=\frac{1}{2} \frac{\varepsilon_{33}^{T} \pi r^{2}}{h}\left(g_{e f f} T_{r} h\right)^{2} \\
=\frac{1}{2} d_{e f f} g_{e f f} T_{r}^{2} \pi r^{2} h
\end{gathered}
$$

It is noted that $d_{e f f} g_{e f f}$ is an important figure of merit that determines the output energy of a radially stressed PZT disk. Because $g=\frac{d}{\varepsilon}$,

$$
\begin{aligned}
d_{e f f} g_{e f f} & =\left(d_{31}+A d_{33}\right) \cdot\left(g_{31}+A g_{33}\right) \\
& =d_{31} g_{31}+A\left(d_{31} g_{33}+d_{33} g_{31}\right)+A^{2} d_{33} g_{33}
\end{aligned}
$$

From Equation (3.28), the products $d_{31} g_{31}, d_{31} g_{33}, d_{33} g_{31}$, and $d_{33} g_{33}$ all have influence on the output energy $W$. The United States (U.S.) Navy established a naming scheme for different types of PZT from Navy type I to VI, and each type is optimally designed for specific applications. The Navy's type designation has been adopted by many PZT vendors like APC Int. (APC-X), PI Ceramic (PIC-X), and Morgan Electro Ceramics (PZT-X). Table 3-2 listed the parameters and the figures of merit of some PZT ceramics, from Navy type I to VI, available from these companies. PZT5J, APC850 and APC855 are labeled as soft piezoelectric materials, while APC840 and APC880 are labeled as hard materials. Among these listed PZT ceramics, the softest ceramic APC855 has the highest figure of merit $d_{e f f} g_{e f f}$, given that its $d_{31} g_{31}, d_{31} g_{33}, d_{33} g_{31}$, and $d_{33} g_{33}$ are all higher than those of the other ceramics.

Curie point of temperature $T_{c}$ is also an essential parameter, above which the PZT ceramic will be depolarized and lose its piezoelectric effect. It is recommended that PZT 
ceramics work under half of the Curie temperature to increase their lifetime [43]. Among these ceramics, APC 855 has the lowest Curie point of $200^{\circ} \mathrm{C}$, and therefore the maximum operating temperature is $100^{\circ} \mathrm{C}$, which is more than enough considering the normal temperature of the asphalt pavement (typically below $60^{\circ} \mathrm{C}$ ). Therefore, APC855 is chosen to be the PZT ceramic material in the Cymbal transducers.

Table 3-2 Parameters of PZT materials of different Navy types.

\begin{tabular}{|c|c|c|c|c|c|c|c|}
\hline \multicolumn{2}{|c|}{$\begin{array}{l}\text { PZT parameter } \\
\text { (Unit) }\end{array}$} & APC840 & APC 850 & APC880 & PIC110 & PZT5J & APC855 \\
\hline \multicolumn{2}{|c|}{$\begin{array}{l}\text { Navy Type } \\
\text { Equivalent }\end{array}$} & I & II & III & IV & $\mathrm{V}$ & VI \\
\hline \multicolumn{2}{|c|}{$\begin{array}{l}\text { Relative Permittivity } \\
\varepsilon_{r}\end{array}$} & 1250 & 1750 & 1000 & 950 & 2750 & 3300 \\
\hline \multicolumn{2}{|c|}{ Curie Point $T_{C}\left({ }^{\circ} \mathrm{C}\right)$} & 325 & 360 & 310 & 150 & 250 & 200 \\
\hline \multirow{2}{*}{$\begin{array}{c}\text { Young's } \\
\text { Modulus } \\
(G P a)\end{array}$} & $Y_{11}^{E}$ & 80 & 63 & 90 & NA & 64.4 & 59 \\
\hline & $Y_{33}^{E}$ & 68 & 54 & 72 & NA & 56.7 & 51 \\
\hline \multirow{2}{*}{$\begin{array}{c}\text { Piezoelectric } \\
\text { Charge } \\
\text { Constant } \\
\left(10^{-12} C / N\right) \\
\end{array}$} & $d_{33}$ & 290 & 400 & 215 & 120 & 554 & 630 \\
\hline & $-d_{31}$ & 125 & 175 & 95 & 50 & 234 & 276 \\
\hline \multirow{2}{*}{$\begin{array}{c}\text { Piezoelectric } \\
\text { Voltage } \\
\text { Constant } \\
\left(10^{-3} \mathrm{Vm} / \mathrm{N}\right) \\
\end{array}$} & $g_{33}$ & 26.5 & 26 & 25 & -11.9 & 22.7 & 21.0 \\
\hline & $-g_{31}$ & 11 & 12.4 & 10 & NA & 9.3 & 9.0 \\
\hline \multicolumn{2}{|c|}{$d_{33} \cdot g_{33}\left(10^{-12}\right)$} & 7.685 & 10.400 & 5.375 & -1.428 & 12.576 & 13.230 \\
\hline \multicolumn{2}{|c|}{$d_{31} \cdot g_{31}\left(10^{-12}\right)$} & 1.375 & 2.170 & 0.950 & NA & 2.176 & 2.484 \\
\hline \multicolumn{2}{|c|}{$d_{33} \cdot g_{31}\left(10^{-12}\right)$} & 3.190 & 4.960 & 2.150 & NA & 5.152 & 5.670 \\
\hline \multicolumn{2}{|c|}{$d_{31} \cdot g_{33}\left(10^{-12}\right)$} & 3.3125 & 4.550 & 2.375 & 0.595 & 5.312 & 5.796 \\
\hline
\end{tabular}




\subsubsection{Selection of Cymbal transducer structural parameters}

The PZT ceramic disks supplied by APC International have an outer diameter available from 0.250 inch $(6.35 \mathrm{~mm})$ to 2.000 inch $(50.8 \mathrm{~mm})$. It is assumed that the vehicle tire contact area is a rectangle of $140 \mathrm{~cm}^{2}-180 \mathrm{~cm}^{2}$ as calculated before. In order to make full use of the tire contact area, a Cymbal array arranged in a container is a reasonable solution.

The diameter of the Cymbal unit is chosen to be 1.00 inch $(25.4 \mathrm{~mm})$ considering the Cymbal endcaps fabrication simplicity and cost. The tire contact has enough space for the

Cymbal array with 20 contact areas $\left(\frac{140 \mathrm{~cm}^{2}}{(2.54 \mathrm{~cm})^{2}}=21.7\right)$, and there should be enough space between each Cymbal unit so as to supply rigid support such that the array will not be overloaded. It is assumed that the load of the tire is about $3600 \mathrm{~N}$, and the load on one Cymbal unit is approximately $180 \mathrm{~N}$. APC 855 can safely withstand $35 \mathrm{MPa}$ in tension [61], and 76.5 MPa in compression (based on the maximum strain 0.15\% [62]). Therefore the Cymbal to be designed should ensure the load of $180 \mathrm{~N}$, and the PZT disk inside should below its tensile and compressive strength limit and generates the most power. Any load higher than $180 \mathrm{~N}$ will be taken by the container.

\subsubsection{Finite Element Analysis (FEA) of the Cymbal transducer}

The FEA model is necessary for determining the geometry of the Cymbal unit, and COMSOL Multiphysics is chosen to be the FEA program. Because the Cymbal transducer is axis-symmetric, a 2D axisymmetric model is made and meshed in 
COMSOL Multiphysics. As shown in Figure 3.11, the load and structure of the Cymbal transducer is also symmetric about the $\mathrm{x}-\mathrm{y}$ plane. The compression to the Cymbal transducer is from the container cover, so a rigid block is modeled as the cover, and a contact pair constraint is set between the block and the endcap apex. The endcap is glued on the PZT disk using epoxy adhesive. The epoxy is modeled with a thickness of $0.1 \mathrm{~mm}$ as measured by experiment, and the following approximate values of general commercial epoxy adhesives are used in the simulation: Young's modulus of $2 \mathrm{GPa}$, Poisson's ratio of 0.37 , and peel shear stress of $24 \mathrm{MPa}$ [63]. The boundary conditions between the endcap and the epoxy and between the epoxy and the PZT disk are set to be continuous identity pairs. A constant axisymmetric boundary load of $180 \mathrm{~N}$ is set on the top surface of the block. A structured fine mesh with triangular elements is generated, and the number of DOFs been solved is 7093 .

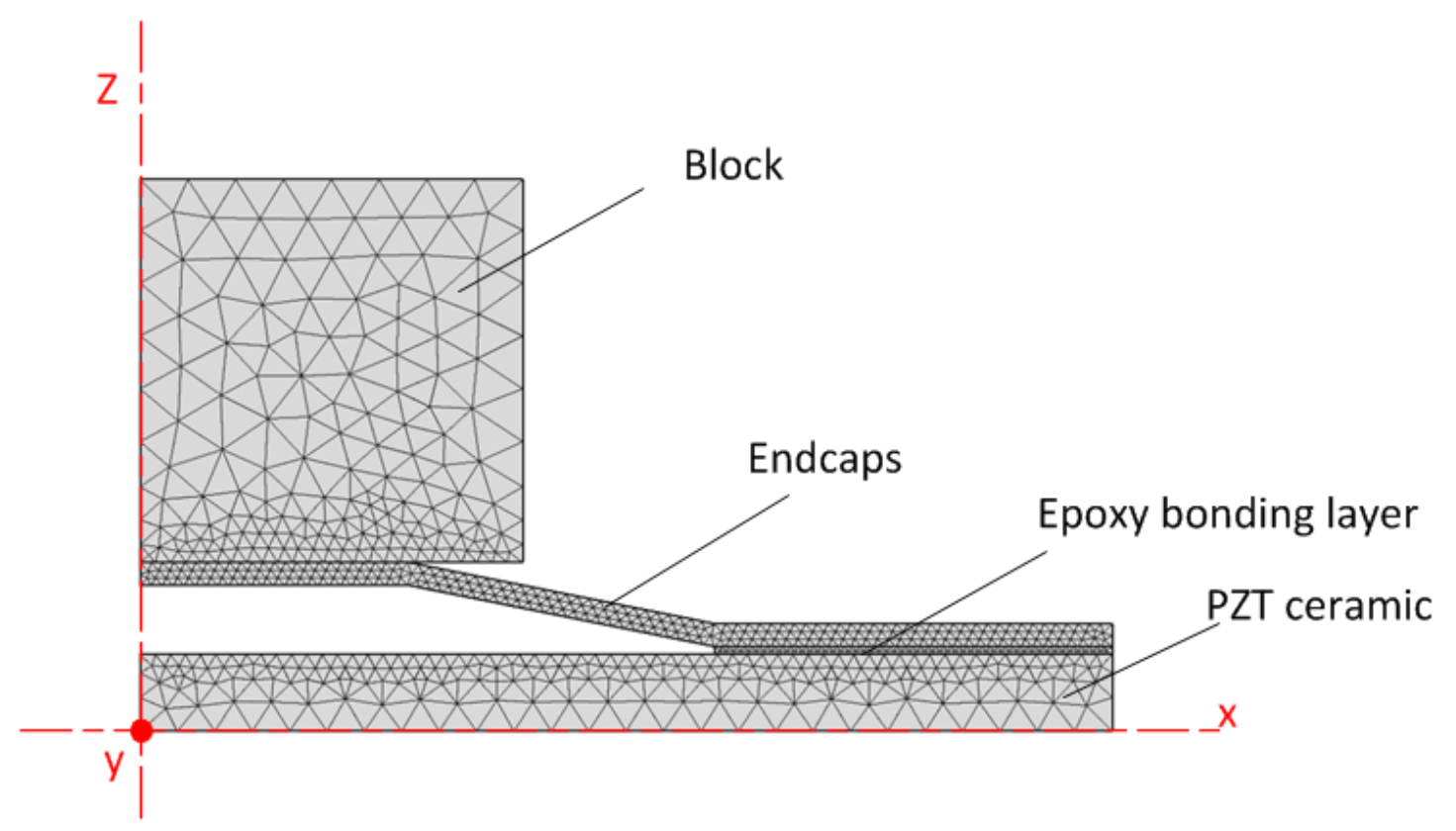

Figure 3.11 Cymbal model in COMSOL Multiphysics. 
The copper alloy phosphor bronze is chosen to be the endcap material for its high machinability, high durability and low cost [64]. The properties of the phosphor bronze are listed in Table 3-3.

Table 3-3 Phosphor bronze properties

\begin{tabular}{|c|c|c|c|c|c|}
\hline Material & Composition & $\begin{array}{c}\text { Density } \\
\left(\mathrm{g} / \mathrm{cm}^{3}\right)\end{array}$ & $\begin{array}{c}\text { Modulus of } \\
\text { Elasticity } \\
(\mathrm{GPa})\end{array}$ & $\begin{array}{c}\text { Yield } \\
\text { Strength } \\
(\mathrm{Mpa})\end{array}$ & $\begin{array}{c}\text { Poisson's } \\
\text { Ratio }\end{array}$ \\
\hline $\begin{array}{c}\text { phosphor } \\
\text { bronze [65] }\end{array}$ & $\begin{array}{c}94.8 \% \mathrm{Cu}, \\
5-6 \% \mathrm{Sn} \\
0.2 \% \mathrm{P}\end{array}$ & 8.86 & 110 & 550 & 0.34 \\
\hline
\end{tabular}

Through the FEA simulation, the stress distribution of the endcaps and PZT disk should be examined so that they will not exceed their stress limit under the load. There are five structural parameters $D_{c}, D_{d}, d_{c}, T_{m}, T_{p}$ (Figure 3.8) that will affect the stress distribution, the endcap apex displacement, and the generated open circuit voltage. In order to investigate the effects of these five parameters respectively, four of them are preset in default values, and then the other one is swept through a range of values. The default values of $D_{c}, D_{d}, d_{c}, T_{m}, T_{p}$ are given in Table 3-4.

Table 3-4 Structural parameters used in FEA simulation

\begin{tabular}{|c|c|c|c|c|c|}
\hline Parameter & $\begin{array}{c}\text { Cavity base } \\
\text { diameter(mm) }\end{array}$ & $\begin{array}{c}\text { Cavity apex } \\
\text { diameter }(\mathrm{mm})\end{array}$ & $\begin{array}{c}\text { Cavity } \\
\text { depth }(\mathrm{mm})\end{array}$ & $\begin{array}{c}\text { Metal endcap } \\
\text { thickness }(\mathrm{mm})\end{array}$ & $\begin{array}{c}\text { PZT } \\
\text { thickness } \\
(\mathrm{mm})\end{array}$ \\
\hline Symbal & $D_{c}$ & $D_{d}$ & $d_{c}$ & $T_{m}$ & $T_{p}$ \\
\hline Value & 15 & 7 & 1 & 0.3 & 2 \\
\hline
\end{tabular}


An extra-fine mesh with triangular elements is generated, and it has 11932 DOFs been solved. Its predicted values are compared with the fine mesh with 7093 DOFs. Table 3-5 shows that the fine mesh with 7093 DOFs essentially gives the same accuracy as 11932 DOFs.

Table 3-5 Comparison between the fine and the extra fine meshes.

\begin{tabular}{|c|c|c|c|}
\hline Predicted Values & $\begin{array}{c}\text { Fine Mesh } \\
\text { (7093 DOFs) }\end{array}$ & $\begin{array}{c}\text { Extra Fine Mesh } \\
\text { (11932 DOFs) }\end{array}$ & Difference (\%) \\
\hline $\begin{array}{c}\text { PZT Maximum Tensile } \\
\text { Stress (MPa) }\end{array}$ & 21.4645 & 21.4938 & 0.1367 \\
\hline $\begin{array}{c}\text { PZT Central Tensile } \\
\text { Stress (MPa) }\end{array}$ & 10.6541 & 10.6547 & 0.005068 \\
\hline $\begin{array}{c}\text { Endcap Maximum Von } \\
\text { Mises Stress (MPa) }\end{array}$ & 161.9057 & 161.9067 & 0.0005744 \\
\hline $\begin{array}{c}\text { Endcap Maximum } \\
\text { Displacement (mm) }\end{array}$ & 0.0421 & 0.0421 & 0.02376 \\
\hline $\begin{array}{c}\text { Block Displacement } \\
\text { (mm) }\end{array}$ & 0.0253 & 0.0253 & 0.03954 \\
\hline Maximum Voltage (V) & 193.9306 & 193.9403 & 0.005017 \\
\hline
\end{tabular}

The FEA model is first simulated using the structural parameters given in Table 3-4 in order to get a brief idea of the stress distribution and the power generated in the Cymbal transducer under the load of $180 \mathrm{~N}$. Von Mises stress is usually used for determining whether a material yields or not and applies best to ductile materials, such as metals, which will experience plastic deformation before failure. On the other hand, brittle materials, such as ceramic, will break abruptly without plastic deformation. Thus, Von Mises stress distribution is simulated in the metal endcap. For the PZT ceramics, the maximum compressive strength $(76.5 \mathrm{MPa})$ is much higher than the maximum tensile strength (35 MPa). Besides, as Von Mises stress only indicates the magnitude without direction, it cannot provide information on whether a material is in compression or 
tension. The first and third principal stress distribution is usually used to locate the maximum compressive and tensile stress in a material. Accordingly, the principal stress is simulated in the PZT ceramic disk and the epoxy adhesive layer.

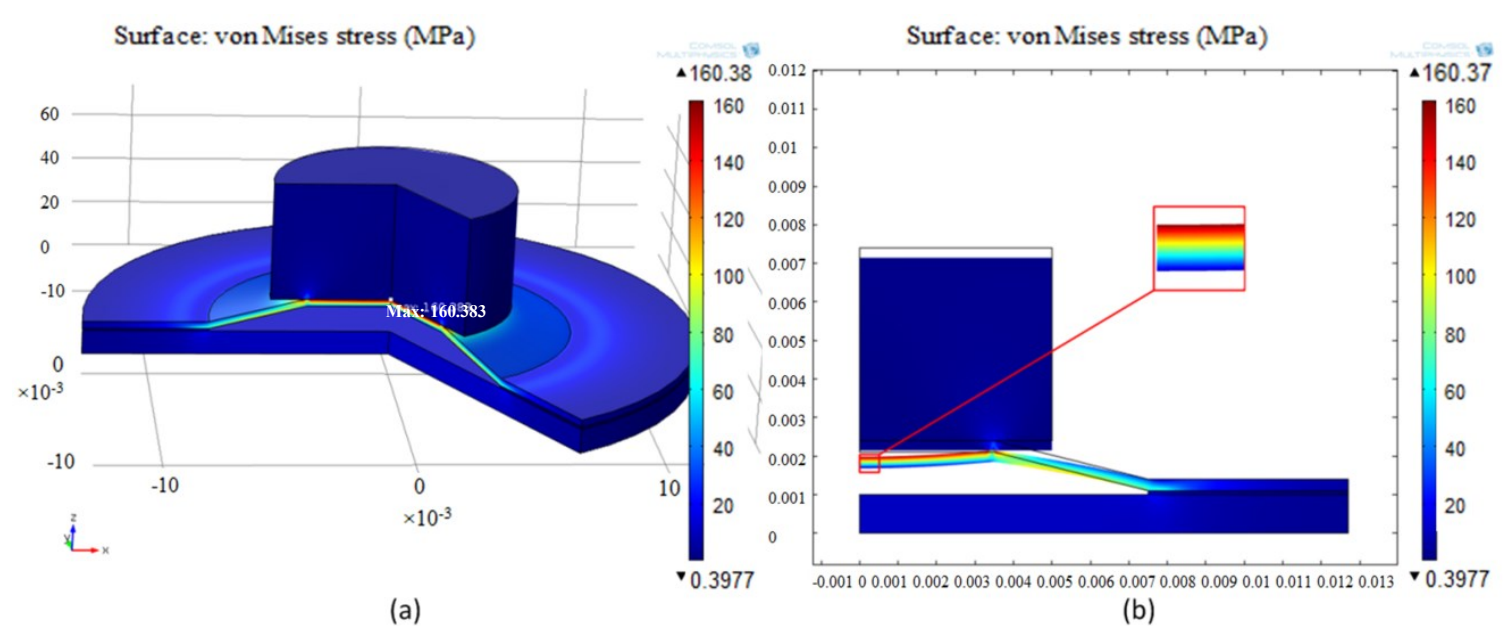

Figure 3.12 Von Mises Stress of Cymbal endcap: (a) Isometric view; (b) Cross-section view.

Figure 3.12 illustrates the Von Mises stress distribution in the endcap of the Cymbal unit in both isometric view and cross-section view. It can be observed that the displacements (amplified in a scale of 10) of the block and the endcap apex are different. That is because the constraint between the block and the endcap apex is a contact pair, and the endcap apex acts like a simply-supported bridge with compressive stress in the upper layer and tensile stress in the lower layer, as shown in Figure 3.12(b). The endcap apex has the tendency to be bent into concave and experiences a larger deformation than the block above. It should be noted that under no circumstance should the endcap apex maximum deformation exceeds the cavity depth. In addition, it can be observed that there is a stress concentration on the endcap apex, so the Von Mises stress in that area should be kept under the phosphor bronze's yield stress (550 MPa). 


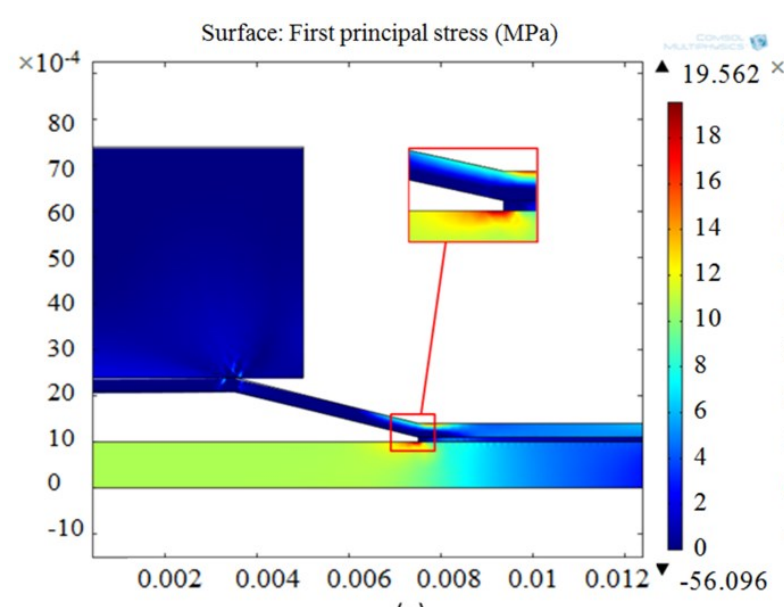

(a)

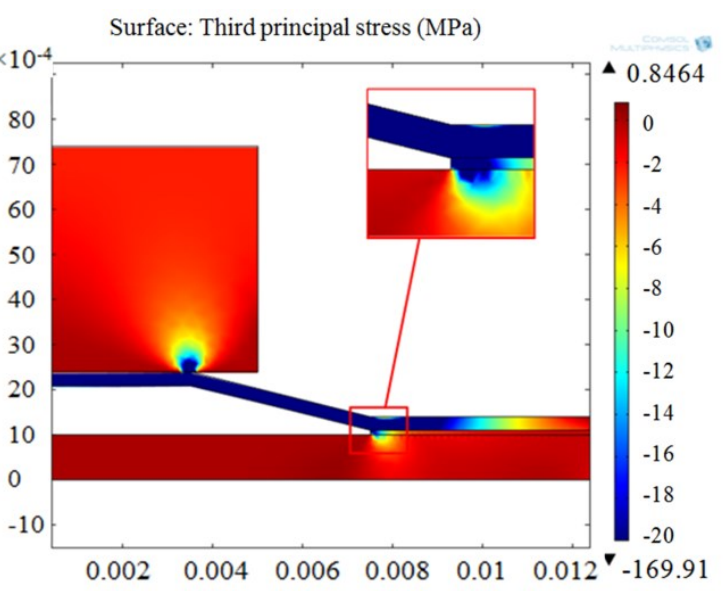

(b)

Figure 3.13 (a) First principal stress (tensile); (b) Third principal stress (compressive).

As illustrated in Figure 3.13, stress concentration of the PZT ceramic can be observed just under the cavity base rim. For the first principal stress distribution in Figure 3.13(a), the maximum tensile stress is the maximum first principal stress (positive). In the third principal stress distribution of Figure 3.13(b), the maximum compressive stress is the minimum third principal stress (negative). It can be noticed that both the maximum compressive and tensile stresses are on the surface of the PZT. The maximum compressive stress is located on the inside edge of the cavity base rim, whereas the maximum tensile stress is located on the outside edge of the cavity base rim. In addition, the first principal stress is positive and uniformly distributed inside the cavity base rim, which proves the assumption made earlier that tensile stress is mainly distributed in the cavity region of the PZT disk. 


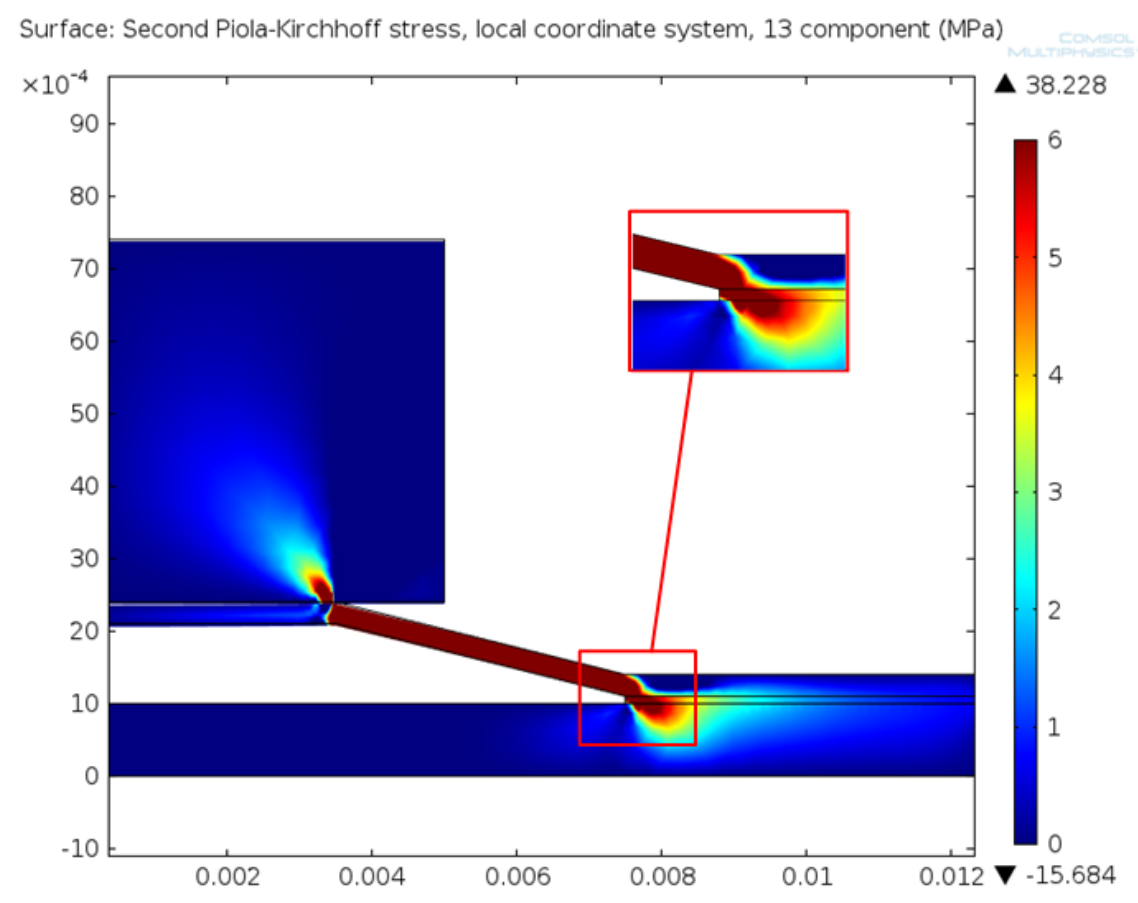

Figure 3.14 Shear stress $T_{31}$ distribution.

Figure 3.14 shows the radial shear stress $T_{31}$ distribution in the cross section of the Cymbal under load. It is found that the maximum shear stress is located on the outside edge of the cavity base rim on the PZT surface. Hence, the maximum shear stress between PZT disk and epoxy adhesive should not exceed the maximum peel shear strength of the commercial epoxy adhesive.

Assuming that the electric potential at the bottom of the PZT is zero, and the PZT disk is not plated with electrodes, then the open circuit voltage generated in the PZT is distributed as shown in Figure 3.15. Inside the cavity base rim, the voltage is uniformly distributed like the first principal stress. The voltage of the PZT disk is highest in the area under the cavity base rim, which is caused by the principal stress concentration in that area. The energy generated is calculated using the open circuit voltage in the central area 
on the PZT surface and the diameter of the cavity base, ignoring the energy generated anywhere else.

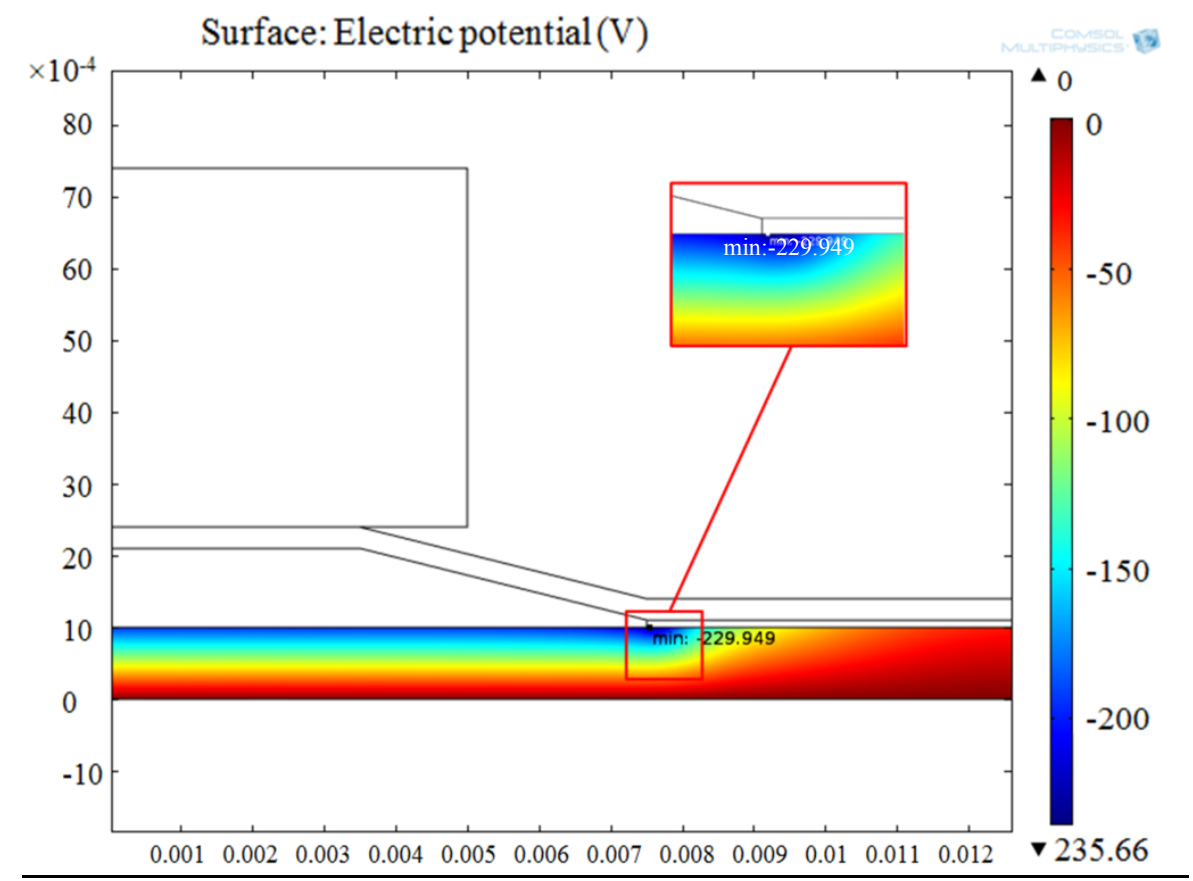

Figure 3.15 Open circuit voltage generated in PZT.

Given all the stress distribution calculated above from the established Cymbal model, it is found that all the maximum stress (compressive stress, tensile stress, shear stress) of the PZT disk are located on its surface near the cavity base rim. In COMSOL, global variable probes are able to extract certain data from certain areas in the model. Therefore, for the structural parameter sweeping simulations in the following sections, global variable probes are placed in the endcaps, in the central area of the PZT disk, on the surface of the PZT disk, and in the rigid block, respectively, to measure the maximum Von Mises stress and displacement of the endcaps, the output voltage of the PZT disk, the maximum first 
principal stress, the third principal stress and shear stress on the PZT disk surface, and the rigid block displacement.

\subsubsection{Effects of the depth of cavity $d_{c}$}

The value of the endcap cavity depth $d_{c}$ is swept from $0.5 \mathrm{~mm}$ to $1.5 \mathrm{~mm}$ with a step of $0.1 \mathrm{~mm}$, while the other structural values are kept as constant (i.e., default values). The trends of all the values of interest (i.e., stresses, displacement, and open circuit voltage) are shown in Figure 3.16. Figure 3.16(a) shows the trends of the PZT central stress, the maximum PZT tensile and compressive stress, and the maximum shear stress on the PZT surface. For this range of $d_{c}$, the stresses in PZT are all inversely proportional to $d_{c}$. The maximum compressive stress is maintained at a level far below the PZT compressive strength $(76.5 \mathrm{MPa})$, and the maximum tensile stress is below the PZT tensile strength (35 MPa) if $d_{c}>0.6 \mathrm{~mm}$. Additionally, the maximum epoxy shear stress is under the epoxy adhesive lap shear strength $(20 \mathrm{MPa})$ if $d_{c}>0.7 \mathrm{~mm}$. The ratio of the PZT central

tensile stress to the maximum PZT tensile stress, $R=\frac{T_{c}}{T_{t}}$, determines how high the central tensile stress can be if the maximum PZT tensile stress is the PZT tensile strength. The higher the ratio, the higher the maximum energy could be generated. In design, this stress ratio $R$ should be kept as high as possible. It is shown that the cavity depth has a slight effect on the stress ratio as it fluctuates between 0.5 and 0.55 with the peak at $d_{c}=1 \mathrm{~mm}$. 

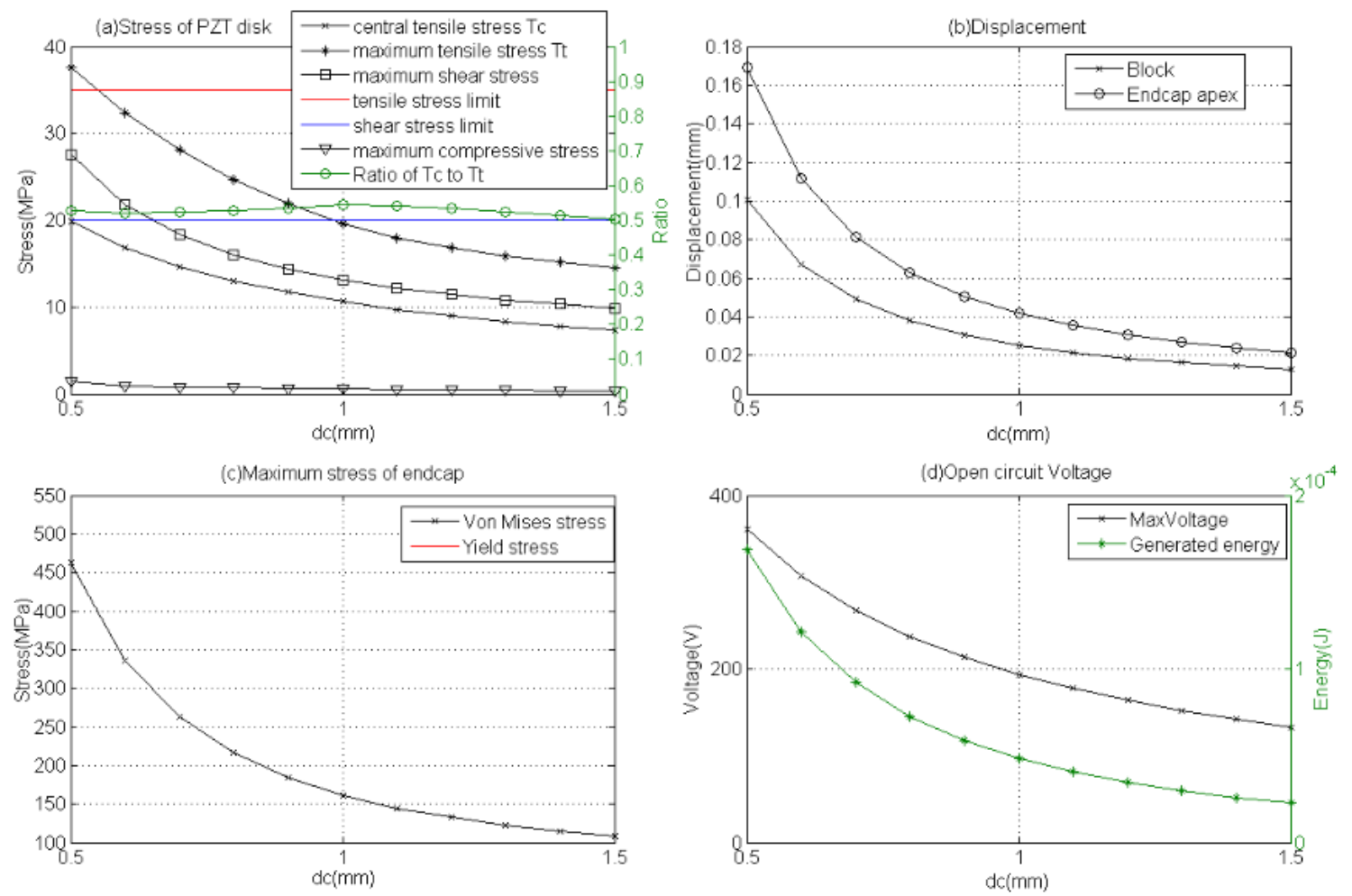

Figure 3.16 Effects of cavity depth $d_{c}$ : (a) Stress of PZT; (b) Displacement of rigid block and endcap; (c) Maximum VonMises Stress of the endcap; (d) Generated voltage and energy in PZT.

Figure 3.16(b) illustrates the displacement trend of the rigid block and the endcap apex, and both of their displacements decrease with the growth of $d_{c}$. Figure 3.16(c) shows that the maximum Von Vises stress in the endcap is inversely proportional to $d_{c}$. It is assumed that the central tensile stress on the PZT disk dominates inside the cavity base rim, and the compressive stress is zero. Equation (3.26) indicates the relationship between the output voltage and the PZT central tensile stress is proportional to the radial stress $T_{r}$ if the thickness of the PZT disk is constant. Therefore in Figure 3.16(d), the output voltage has the same trend as the central tensile stress and declines the same way as the central tensile stress with the increase of $d_{c}$. It should be noted that the generated 
voltage is sampled inside the cavity base rim, while the generated voltage is lower outside of the rim, so the sampled voltage will be higher than the open circuit voltage measured in real tests. The generated energy is calculated by Equation (3.27), and it also decreases given a constant capacitance $C$.

Conclusively speaking, the rise in $d_{c}$ will lead to a decrease in the output voltage due to the decline in the tensile stress in the PZT disk. The stress and displacement in the endcap will decrease as well. With $D_{c}=15 \mathrm{~mm}, D_{d}=7 \mathrm{~mm}, T_{m}=0.3 \mathrm{~mm}, T_{p}=2 \mathrm{~mm}, d_{c}$ needs to be over $0.7 \mathrm{~mm}$ in order to guarantee that the PZT and the endcap are below their stress limits. In addition, $d_{c}$ is not recommended to be set too high as the stress ratio and the output power will decrease as it goes higher.

\subsubsection{Effects of the cavity apex diameter $D_{d}$}

The value of the endcap cavity depth $D_{d}$ is swept from $4 \mathrm{~mm}$ to $10 \mathrm{~mm}$ with a step of 1 $\mathrm{mm}$, while the other structural values are kept at defaults. Figure 3.17(a) shows that the PZT central stress, the maximum PZT tensile and compressive stress, and the maximum epoxy shear stress all decrease linearly with the increase of $D_{d}$. For this range of $D_{d}$, the PZT maximum compressive stress is much smaller than the PZT compressive strength, and the maximum PZT tensile and shear stresses are all below their stress limits. The tensile stress ratio increases from 0.5 to 0.55 before $D_{d}$ grows to $7 \mathrm{~mm}$ and then decreases afterward. Figure 3.17(b) illustrates that both of the displacement of the block and the endcap apex decrease with the growth of $D_{d}$, and Figure 3.17(c) shows that the maximum Von Vises stress in the endcap declines as $D_{d}$ grows. Figure 3.17(d) shows 
that the variations of the output voltage with the increase of $D_{d}$. The generated energy is calculated using Equation (3.27), and it also decreases given constant capacitance $C$ in this case.
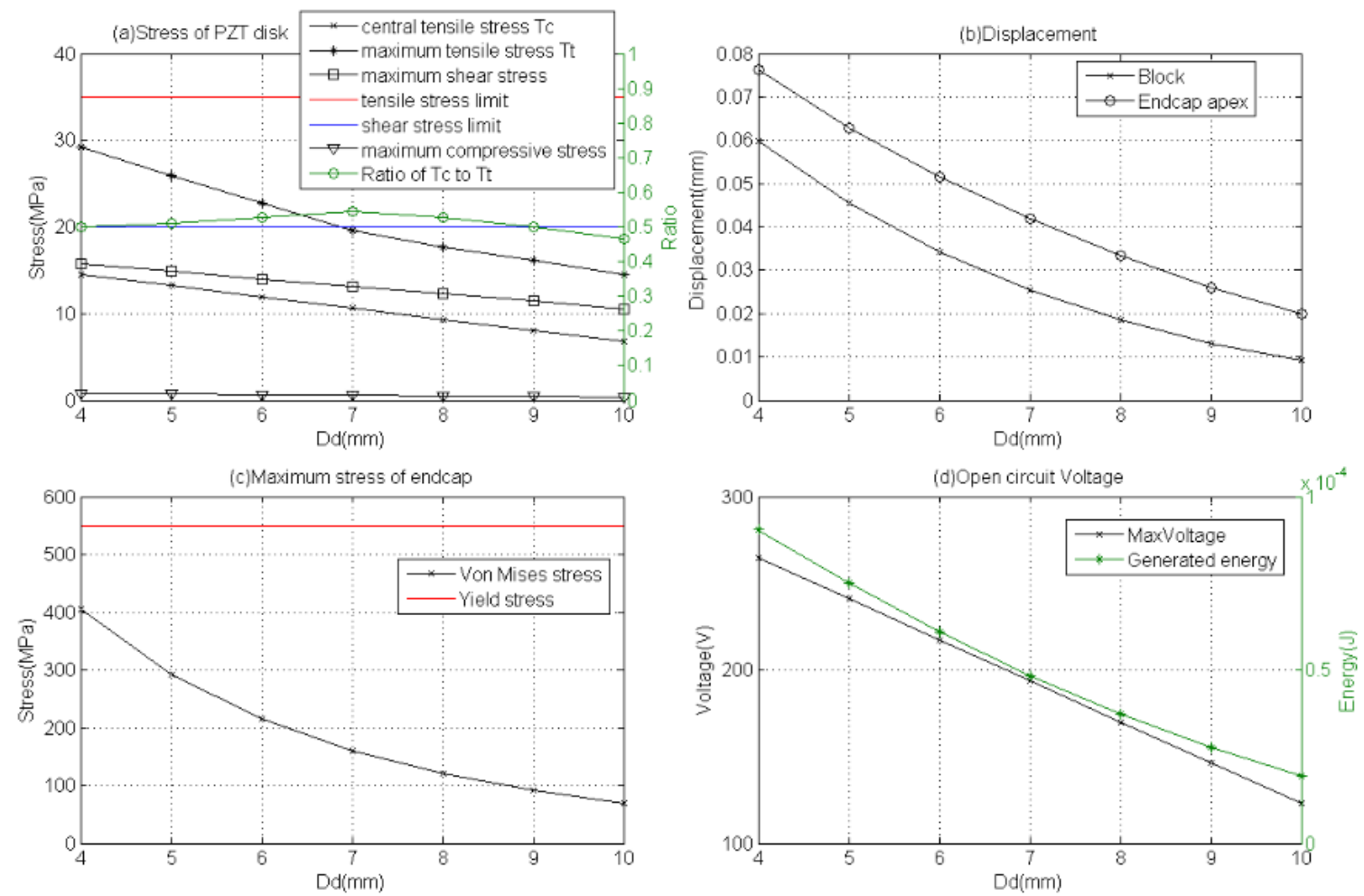

Figure 3.17 Effects of cavity apex diameter $D_{d}$ : (a) Stress of PZT; (b) Displacement of rigid block and endcap; (c) Maximum VonMises Stress of the endcap; (d) Generated voltage and energy in PZT.

In conclusion, the rise of $D_{d}$ will make the output voltage decrease because the tensile stress declines in the PZT disk, and accordingly, the stress and displacement in the endcap will decrease as well. With $D_{c}=15 \mathrm{~mm}, d_{c}=1 \mathrm{~mm}, T_{m}=0.3 \mathrm{~mm}, T_{p}=2 \mathrm{~mm}$, the endcap and PZT disk are safe if $D_{d}$ is over $4 \mathrm{~mm}$. Like $d_{c}, D_{d}$ cannot be set too high considering that it will affect the output power significantly and the stress ratio. 


\subsubsection{Effects of the cavity base diameter $D_{c}$}

The value of the cavity base diameter $D_{c}$ is swept from $11 \mathrm{~mm}$ to $21 \mathrm{~mm}$ with a step of $1 \mathrm{~mm}$, and the other structural values are kept at default. Figure 3.18(a) shows that the PZT central stress, the maximum PZT tensile and compressive stresses increase as $D_{c}$ increases. The epoxy shear stress fluctuates between 10 and $13 \mathrm{MPa}$ when $D_{c}<18 \mathrm{~mm}$ and starts to increase afterward. The PZT maximum compressive stress is still much smaller than the PZT compressive strength, and the maximum PZT tensile and epoxy shear stress are both below their stress limits when $D_{c}<20 \mathrm{~mm}$. The stress ratio $R$ has a peak of 0.56 when $D_{c}$ is between $14 \mathrm{~mm}$ and $16 \mathrm{~mm}$ and keeps decreasing thereafter to 0.4 . Figure 3.17(b) illustrates that both of the displacement of the block and the endcap apex increase with the growth of $D_{d}$, and Figure 3.17(c) shows that the maximum Von Vises stress in the endcap increases as $D_{c}$ grows. Figure $3.17(\mathrm{~d})$ shows that the output voltage, like the PZT central stress, increases linearly with the increase of $D_{c}$. The generated

energy, calculated using $\frac{1}{2} C V^{2}$ and $C=\frac{\varepsilon_{33}^{T} \pi\left(D_{C} / 2\right)^{2}}{h}$, increases dramatically as both the central stress $T_{r}$ and the cavity diameter $D_{c}$ increases. 

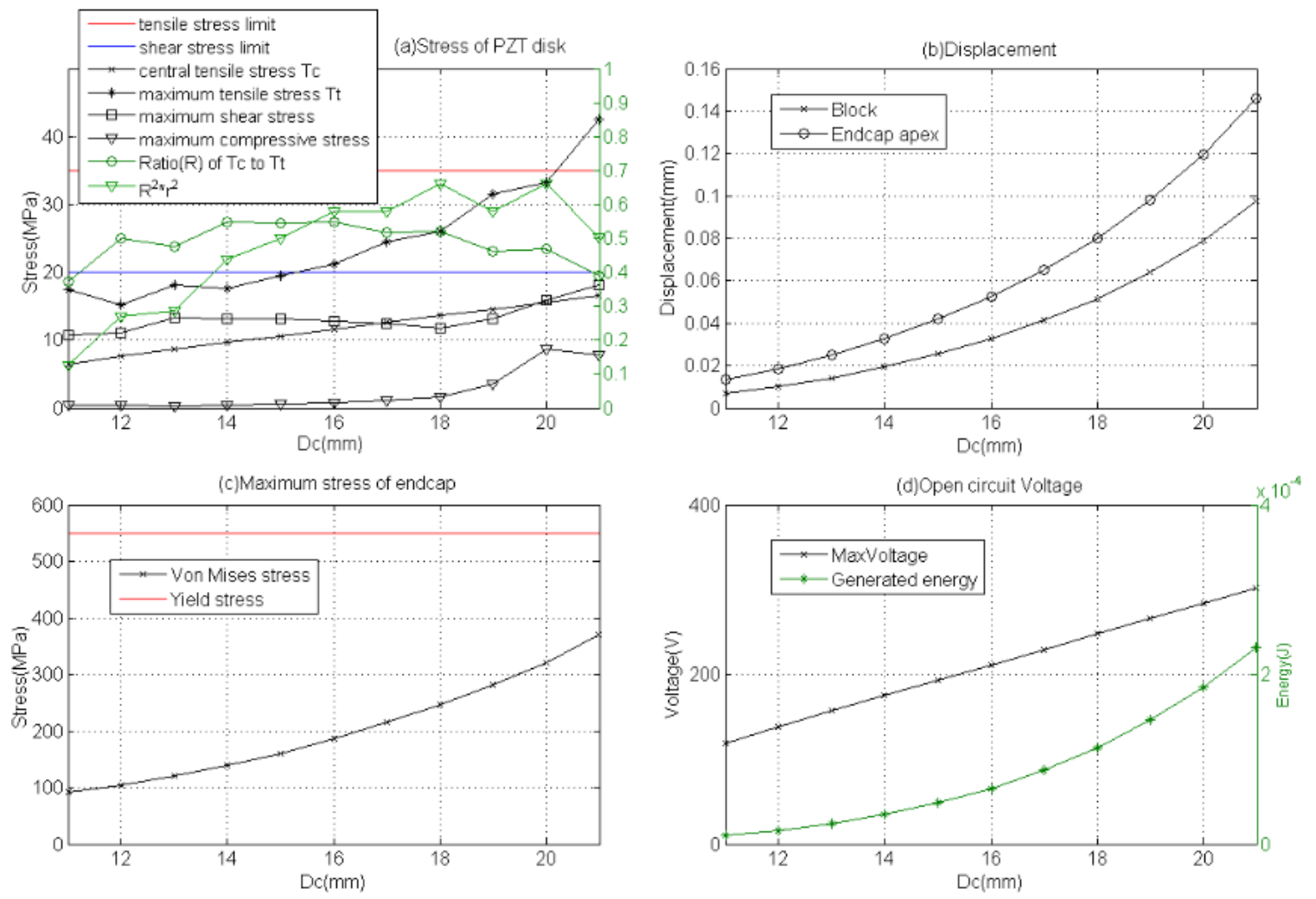

Figure 3.18 Effects of the cavity base diameter $D_{c}$.

Therefore, with $D_{d}=7 \mathrm{~mm}, d_{c}=1 \mathrm{~mm}, T_{m}=0.3 \mathrm{~mm}, T_{p}=2 \mathrm{~mm}, D_{c}$ is suggested to be below $20 \mathrm{~mm}$ in order to keep the PZT and epoxy working under their stress limits (i.e., the tensile and compressive stress limits for the PZT, and the shear stress limit for the epoxy) and in the meantime, obtain the highest output energy. In practice, both conductive and insulated epoxies have a width of at least $2 \mathrm{~mm}$ after being pressed for curing, which means $D_{c}$ cannot be larger than $17 \mathrm{~mm}$. From Equation (3.27), the maximum achievable energy is $W=\frac{1}{2} d_{e f f} g_{e f f}\left(R T_{s}\right)^{2} \pi r^{2} h$. If $d_{\text {eff }} g_{\text {eff }}, h$ and $T_{s}$ (tensile strength) are preset, the maximum achievable energy is proportional to the magnitude $R^{2} r^{2}$. In Figure 3.18(a), the trend of $R^{2} r^{2}$ (scaled) shows that the maximum 
achievable energy has a peak when $D_{c}$ is $16 \mathrm{~mm}$ (before $17 \mathrm{~mm}$ ). Accordingly, the endcap cavity diameter $D_{c}$ is chosen to be $16 \mathrm{~mm}$.

\subsubsection{Effects of the metal endcap thickness $T_{m}$}
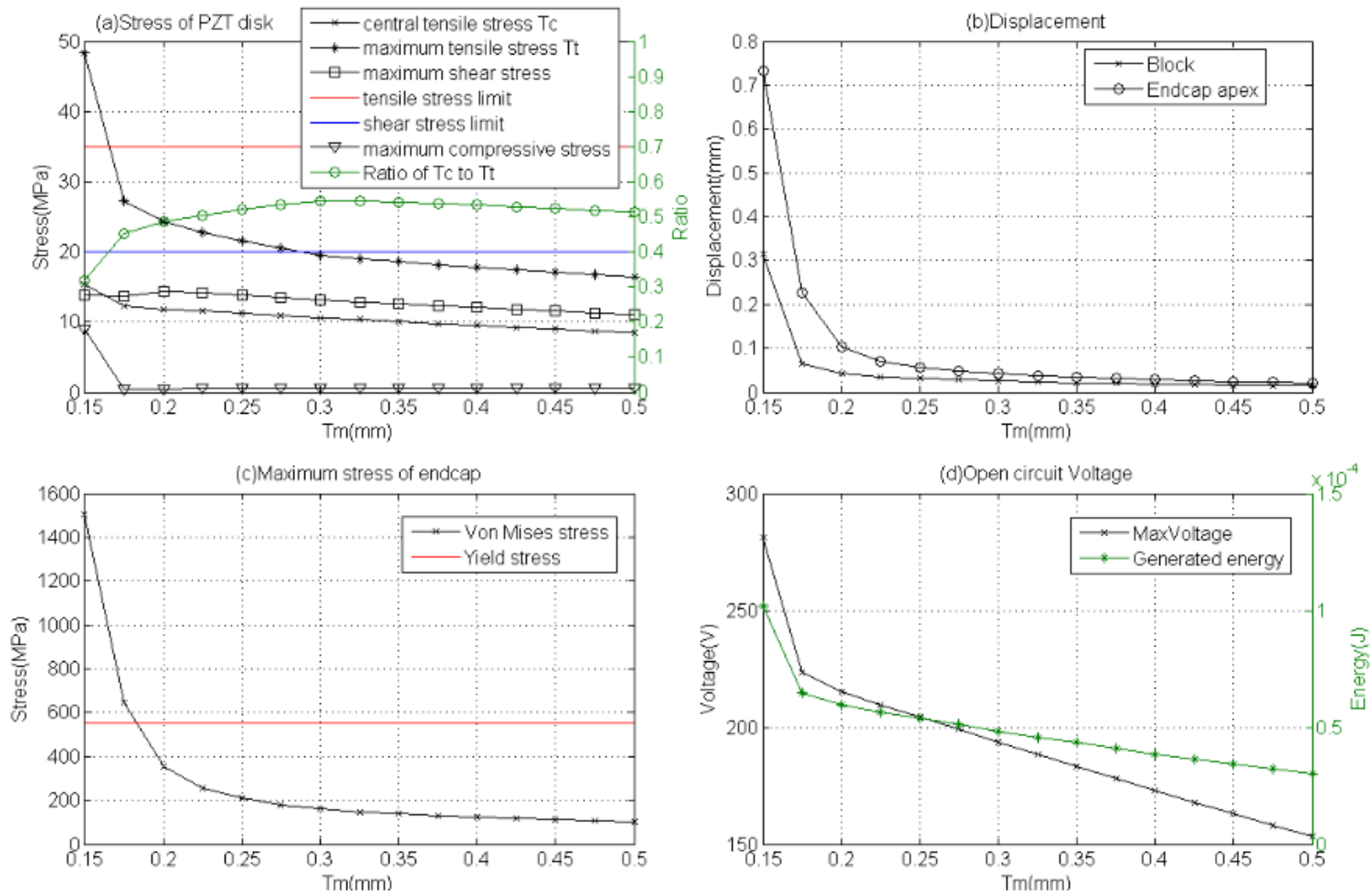

Figure 3.19 Effects of the metal endcap thickness $T_{m}$.

The value of the metal endcap thickness $T_{m}$ is swept from $0.15 \mathrm{~mm}$ to $0.5 \mathrm{~mm}$ with a step of $0.025 \mathrm{~mm}$, while the other structural parameters are kept at default. Figure 3.19(a) shows that as $T_{m}$ grows prior to $0.175 \mathrm{~mm}$, the PZT central stress, the maximum tensile stress and compressive stress in PZT decrease dramatically, while the maximum epoxy shear stress remains almost the same. After $T_{m}$ grows over $0.175 \mathrm{~mm}$, all the mentioned stresses decline slowly. The stress ratio increases largely at first and maintains over 0.5 
when $T_{m}$ is over $0.25 \mathrm{~mm}$. Figure 3.19(b) and (c) shows the block and cavity apex displacement and the endcap maximum Von Mises stress decrease with $T_{m}$. In Figure 3.19(d), the voltage has the same trend as the PZT central stress, and the generated energy decreases along with the voltage.

Therefore, with $D_{d}=7 \mathrm{~mm}, d_{c}=1 \mathrm{~mm}, D_{c}=15 \mathrm{~mm}, T_{p}=2 \mathrm{~mm}, T_{m}$ should be over $0.2 \mathrm{~mm}$ in order to keep the endcap and PZT working under their stress limits and below $0.3 \mathrm{~mm}$ to keep the stress ratio high. Accordingly, $T_{m}$ is chosen to be $0.254 \mathrm{~mm}$ (i.e., $0.01 \mathrm{inch}$ ), which is readily available from Mcmaster-Carr [65].

\subsubsection{Effects of the PZT disk thickness $T_{p}$}

The value of the metal endcap thickness $T_{p}$ is swept from $1 \mathrm{~mm}$ to $5 \mathrm{~mm}$ with a step of $0.5 \mathrm{~mm}$, while the other structural parameters are kept at their default values. Figure 3.20(a) shows that the tensile stress in PZT decreases with $T_{p}$, while the maximum PZT compressive stress and epoxy shear stress remain almost the same. The stress ratio $R$ keeps decreasing from 0.7 to 0.3 as $T_{p}$ grows from $1 \mathrm{~mm}$ to $5 \mathrm{~mm}$. From Equation (3.27), the maximum achievable energy is $W=\frac{1}{2} d_{e f f} g_{e f f}\left(R T_{s}\right)^{2} \pi r^{2} h$. Assuming that $d_{e f f} g_{e f f}, T_{S}$ and $r$ are preset and $h=T_{p}$, the maximum energy is proportional to the magnitude $R T_{p}$. Figure 3.20(b) shows that the displacement of the block and endcap decreases slightly with the variation in $T_{p}$, and Figure $3.20(\mathrm{c})$ illustrates that the change of the maximum Von Mises stress in the endcap is negligible. In Figure 3.20(d), the open circuit voltage slightly increases though the PZT central tensile stress decreases, because 
the capacitance of the PZT $\left(C=\frac{\varepsilon_{33}^{T} \pi\left(D_{C} / 2\right)^{2}}{T_{p}}\right)$ also decreases with the growth of $T_{p}$. The generated energy decreases as the tensile stress affects the open circuit voltage more significantly than the PZT disk thickness based on Equation (3.27).
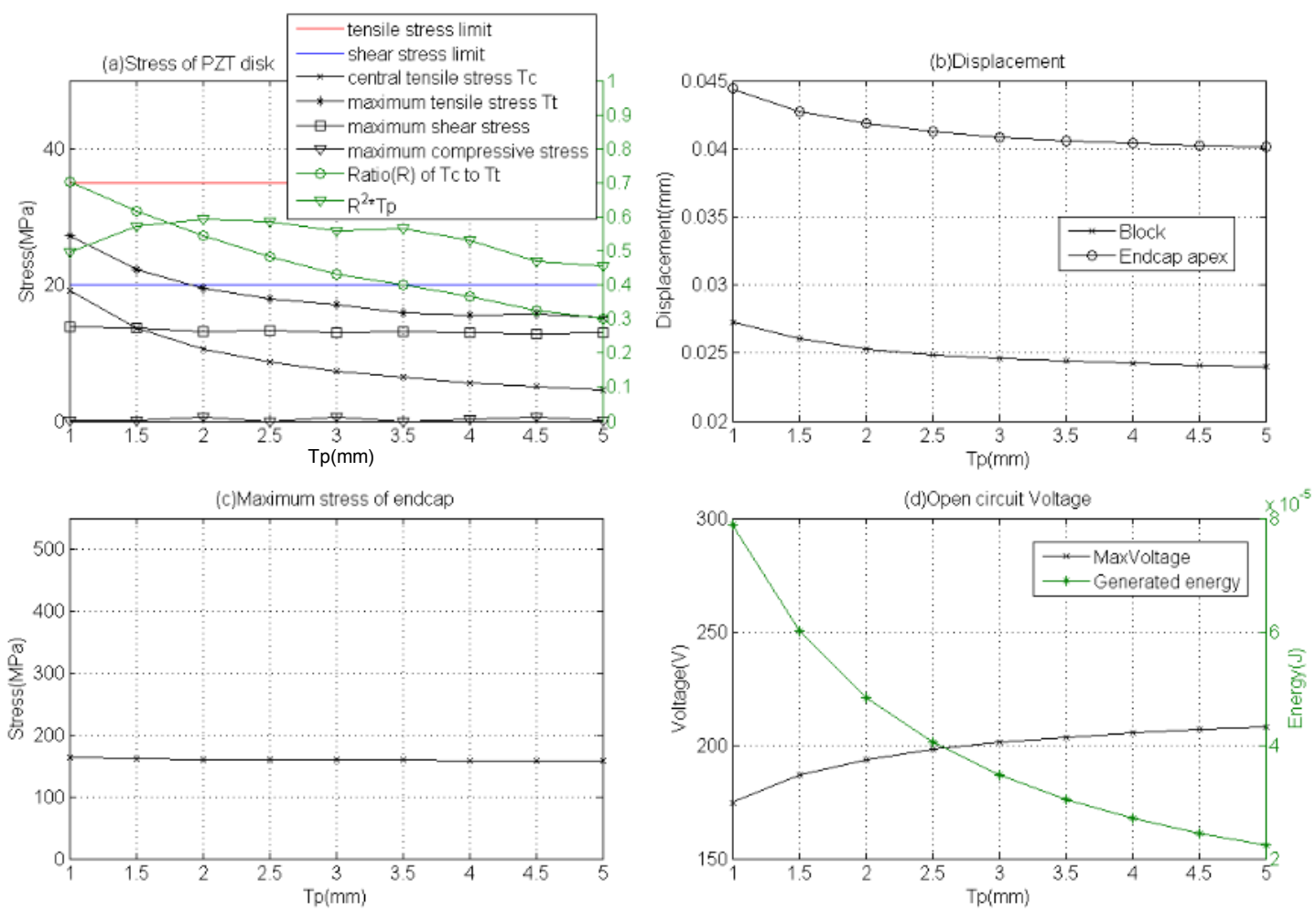

Figure 3.20 Effects of the PZT disk thickness $T_{p}$.

Therefore, the growth of $T_{p}$ has a little effect on the maximum compressive stress and shear stress in the PZT disk, and the maximum Von Mises stress in the endcap; it will lead to a decrease in the PZT tensile stress and the stress ratio. Based on Equation (3.27), if $d_{e f f} g_{e f f}, f, r$ are predetermined, the maximum achievable energy is proportional to the magnitude $R^{2} T_{p}$. In Figure 3.20(a), the trend of $R^{2} T_{p}$ shows that the maximum achievable energy increases as $T_{p}$ grows to $2 \mathrm{~mm}$, but it starts to decrease even with a 
thicker PZT disk thereafter. Accordingly, the thickness of the PZT disk $T_{p}$ is chosen to be $2 \mathrm{~mm}$.

\subsubsection{Effects of both $D_{d}$ and $d_{c}$}

As discussed above, $D_{c}, T_{m}$ and $T_{p}$ are set to be $16 \mathrm{~mm}, 0.254 \mathrm{~mm}$ and $2 \mathrm{~mm} . D_{d}$ and $d_{c}$ are swept together to find an appropriate combination that has the highest tensile stress in the PZT disk under the force of $180 \mathrm{~N}$, while keeping the PZT, epoxy and metal endcap under their stress limits.

Figure 3.21 shows the stresses in the Cymbal unit with $D_{d}$ swept from $5 \mathrm{~mm}$ to $10 \mathrm{~mm}$ with a step of $1 \mathrm{~mm}$ and $d_{c}$ swept from $0.7 \mathrm{~mm}$ to $1.1 \mathrm{~mm}$ with a step of $0.05 \mathrm{~mm}$. $D_{d}$ and $d_{c}$ are chosen to be $6 \mathrm{~mm}$ and $0.8 \mathrm{~mm}$ respectively, where the PZT maximum tensile stress (34.57 MPa) is just below the PZT tensile strength (35 MPa) in Figure 3.21(a). The PZT central stress is $17.63 \mathrm{MPa}$ (Figure 3.21(b)), which makes the stress ratio to be 0.504 . The Von Mises stress (504 MPa) in the endcap is below its yield strength (550 MPa shown in Figure 3.21(c)), and the epoxy maximum stress (17.13 MPa shown in Figure 3.21(d)) is also below the epoxy lap shear stress (20 MPa). The selected values for the key structural parameters are summarized in Table 3-6. 

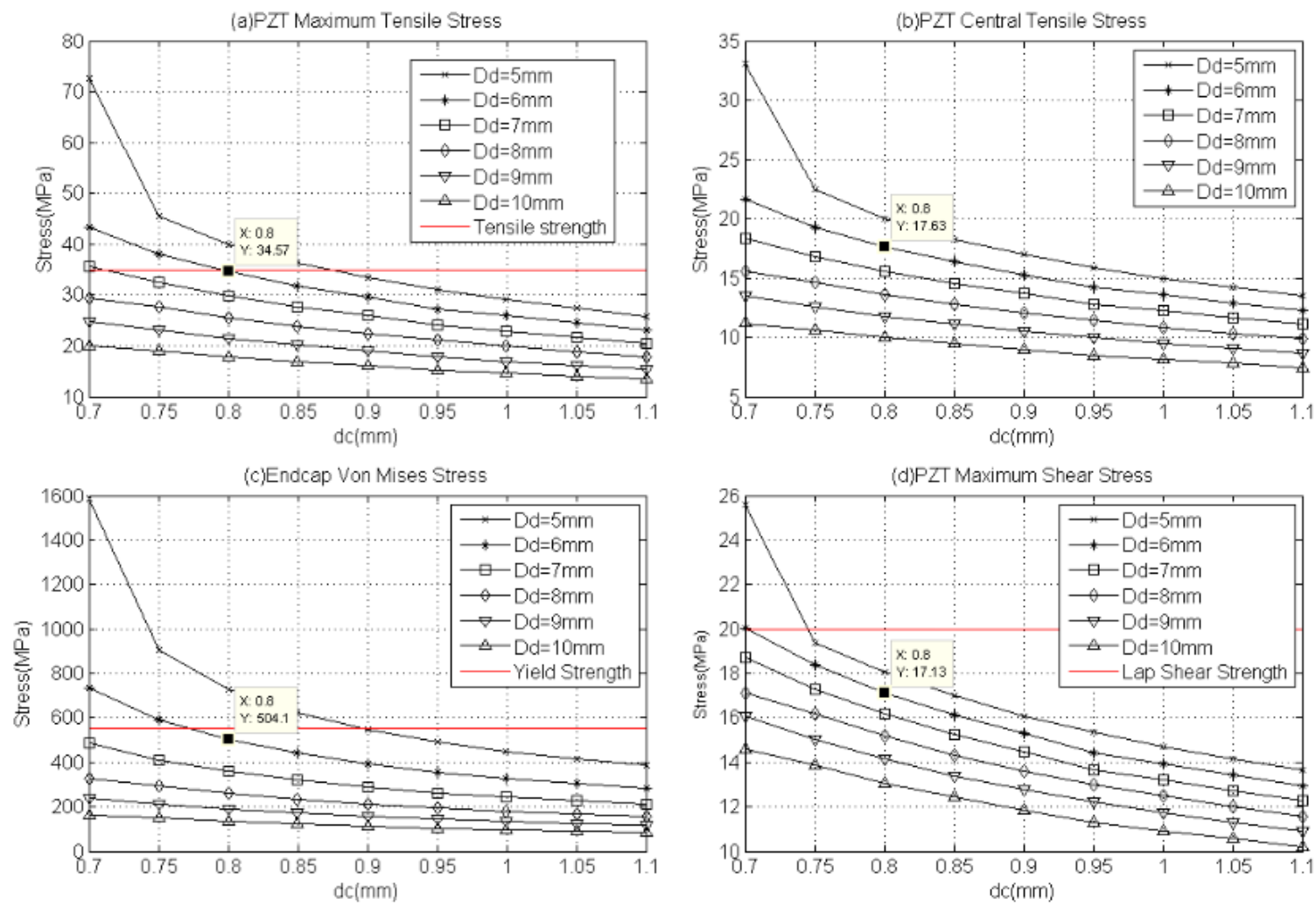

Figure 3.21 Stresses in Cymbal: (a) PZT maximum Tensile Stress; (b) PZT Central Tensile Stress; (c) Endcap Von Mises Stress; (d)PZT Maximum Shear Stress.

Table 3-6 Structural parameters selection

\begin{tabular}{|c|c|c|c|c|c|}
\hline Parameter & $d_{c}(\mathrm{~mm})$ & $D_{d}(\mathrm{~mm})$ & $D_{c}(\mathrm{~mm})$ & $T_{m}(\mathrm{~mm})$ & $T_{p}(\mathrm{~mm})$ \\
\hline Selected & 0.8 & 6 & 16 & 0.254 & 2 \\
\hline
\end{tabular}




\subsection{Cymbal Transducer Fabrication}

The fabrication of the Cymbal transducer is composed of two steps: the fabrication of the Cymbal metal endcaps and the bonding between the endcaps and the PZT disks.

\subsubsection{Cymbal endcap Fabrication}

Manufacturing truncated-conical endcaps using Computer Numerical Control (CNC) machine is difficult and expensive. To fabricate consistent endcaps in a large quantity, a die is designed and bolted on a die set as shown in Figure 3.22. Assuming the spring back of the endcap cavity depth is approximately $30 \%$, the depth of the cavity of the bottom die is machined by a CNC machine to 0.045 inches $(1.143 \times 70 \%=0.8 \mathrm{~mm})$, and the structural details are provided in Appendix A.

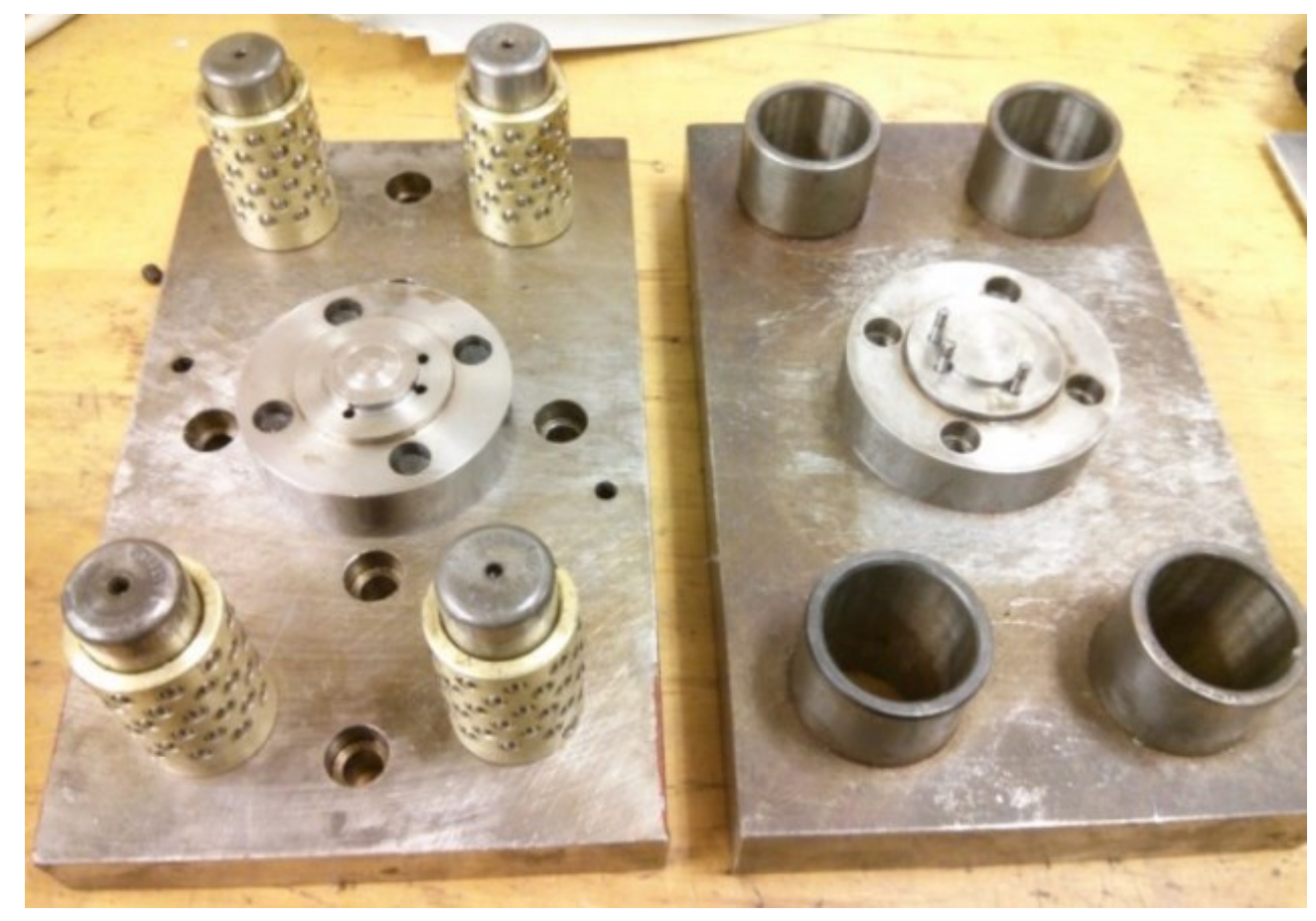

Figure 3.22 Die set top section (left) and base section (right). 
First, the phosphor bronze disk of 1 inch in diameter is punched using an off-the-shelf jewelry punch set. Then, the punched disk is filed by a fine file to remove the burr on its edge. The disk is then placed on the bottom die and positioned by three guiding pins. The die set is punched by a hand punch (shown in Figure 3.23) to shape the disk into concave.

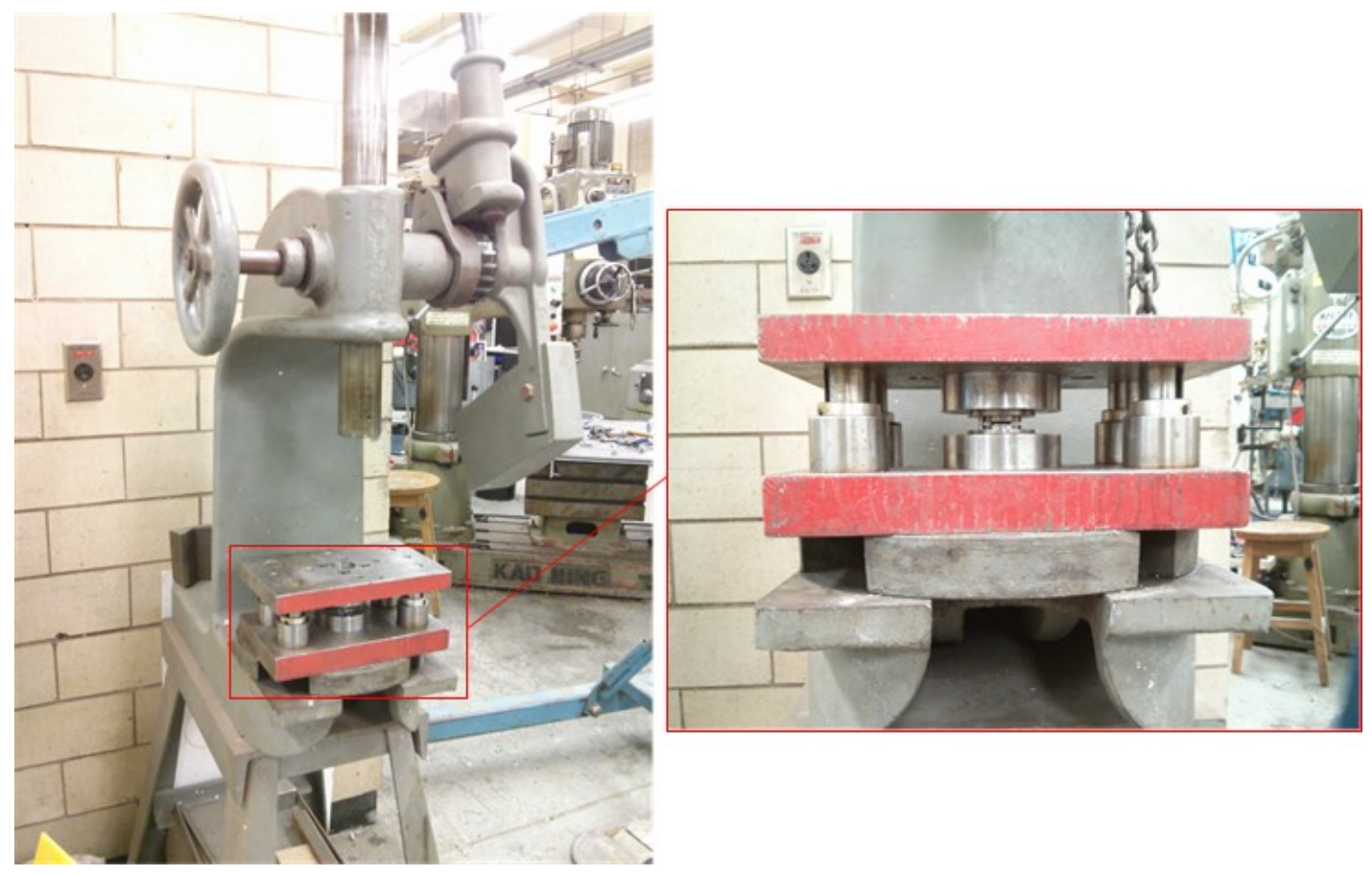

Figure 3.23 The hand punch and die set mounted.

After the punching process, the dimensions of the endcap are measured and compared with the designed dimensions in Table 3-7. It is found that the depth of the cavity $d_{c}$ is $0.5 \mathrm{~mm}$ shallower than expected, and the diameter of the endcap apex $D_{d}$ is $1 \mathrm{~mm}$ wider. The Cymbal model with the measured dimensions is simulated in COMSOL by sweeping force between $160 \mathrm{~N}$ to $220 \mathrm{~N}$ and the results are illustrated in Figure 3.24. 
Table 3-7 Designed and measured dimensions of Cymbal endcap

\begin{tabular}{|c|c|c|c|c|c|}
\hline Parameter & $d_{c}(\mathrm{~mm})$ & $D_{d}(\mathrm{~mm})$ & $D_{c}(\mathrm{~mm})$ & $T_{m}(\mathrm{~mm})$ & $T_{p}(\mathrm{~mm})$ \\
\hline Designed & 0.8 & 6 & 16 & 0.254 & 2 \\
\hline Measured & 0.75 & 7 & 16 & 0.254 & 2 \\
\hline
\end{tabular}

Compared with the designed Cymbal, the fabricated Cymbal needs more force (190N) to get the highest energy. Under the load of $190 \mathrm{~N}$, the PZT central tensile stress is 17.93 $\mathrm{MPa}$, and the maximum PZT tensile stress is at $34.74 \mathrm{MPa}$ (Figure 3.24(a)). Under the load of $180 \mathrm{~N}$ the PZT central tensile stress is $16.81 \mathrm{MPa}$, and the maximum PZT tensile stress is $32.46 \mathrm{MPa}$ (Figure 3.24(a)). From Figure 3.24(b), the rigid block displacement is $0.0745 \mathrm{~mm}$ at $180 \mathrm{~N}$ and $0.08073 \mathrm{~mm}$ at $190 \mathrm{~N}$. Therefore, for the whole Cymbal unit with two endcaps the total displacement is $0.149 \mathrm{~mm}$ at $180 \mathrm{~N}$ and $0.161 \mathrm{~mm}$ at $190 \mathrm{~N}$. The Von Mises stress in the endcap is lowered to $411 \mathrm{MPa}$ under $180 \mathrm{~N}$ due to the increase of $D_{d}$ as shown in Figure 3.24(c). As a result, the fabricated Cymbal is able to work safely under $180 \mathrm{~N}$. The generated energy from the fabricated Cymbal is slightly below that from the designed Cymbal under the same load $180 \mathrm{~N}$ (PZT central stress is 16.81 MPa for the fabricated Cymbal compared to $17.63 \mathrm{MPa}$ for the designed Cymbal). 

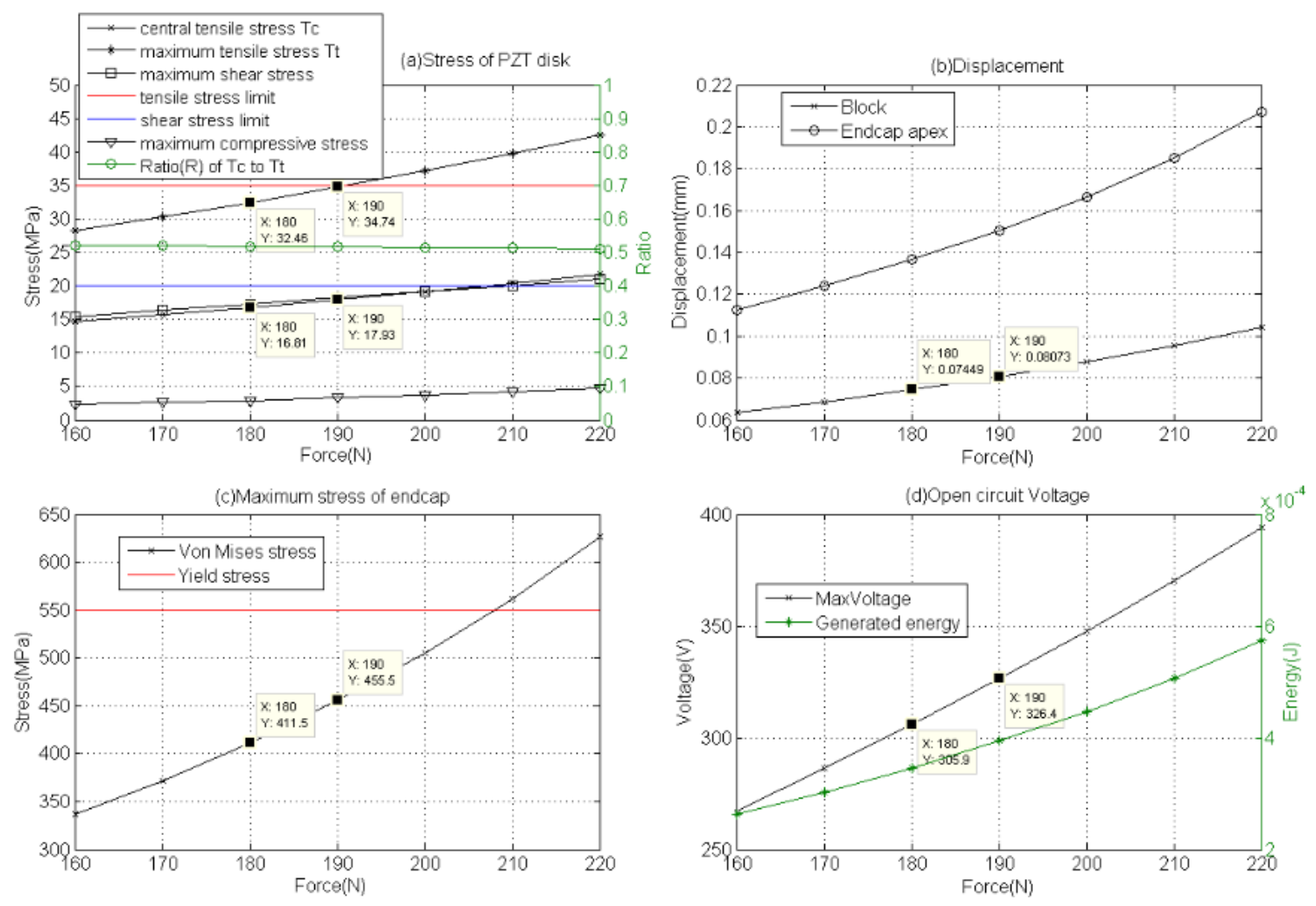

Figure 3.24 Force sweep of the measured model.

\subsubsection{Bonding process}

There are two possible bonding methods for Cymbals: solder bonding and epoxy bonding. The solder bonding method requires heating the PZT disk to the soldering alloy melting temperature $\left(100{ }^{\circ} \mathrm{C}-300{ }^{\circ} \mathrm{C}\right)$, which will affect the PZT ceramic's behavior since this temperature will go over its maximum operating temperature (half of the Curie point). Comparatively, the epoxy adhesive is able to cure under room temperature and does not cause any overheating effects on the PZT material, and therefore epoxy bonding is chosen to be the bonding method for the bonding layer between the endcap and the PZT disk. 
Two different epoxy adhesives are applied for bonding in this work: insulating structural epoxy and silver conductive epoxy. The insulating epoxy, "Professional Speed Set Epoxy" from LEPAGE, has large lap shear strength $(20 \mathrm{MPa})$ and is used to transfer the stress from the endcap to the PZT disk. The silver conductive epoxy, MG Chemicals epoxy $8331 \mathrm{~S}$, has good electrical conductivity $(0.0060 \Omega \cdot \mathrm{cm})$ but low lap shear strength (1 MPa), so it is employed for the electrical connection between the PZT electrode and its endcap. Both epoxy glues are composed of epoxy resin and epoxy hardener. When the resin and hardener are mixed 50/50, it takes approximately 24 hours at room temperature to cure completely.

Figure 3.25 shows the process of the Cymbal fabrication. Firstly, both the endcap and the PZT are polished by ultra-fine sandpaper to remove the oxide layer and are cleaned with alcohol (Figure 3.25(a)). Secondly, the mixture of silver conductive epoxy resin and hardener is applied on two spots of the endcap, as shown in Figure 3.25(b), to ensure conductivity. The mixture of the insulating epoxy resin and hardener is applied on the left area of the contact surface. Then, the PZT disk are sandwiched between two endcaps and clamped by several clips for 24 hours until both epoxy adhesives in it are fully cured (Figure 3.25(c)). Finally, the electrode wires are connected to the electrodes by silver conductive epoxy (Figure 3.25(d)). 

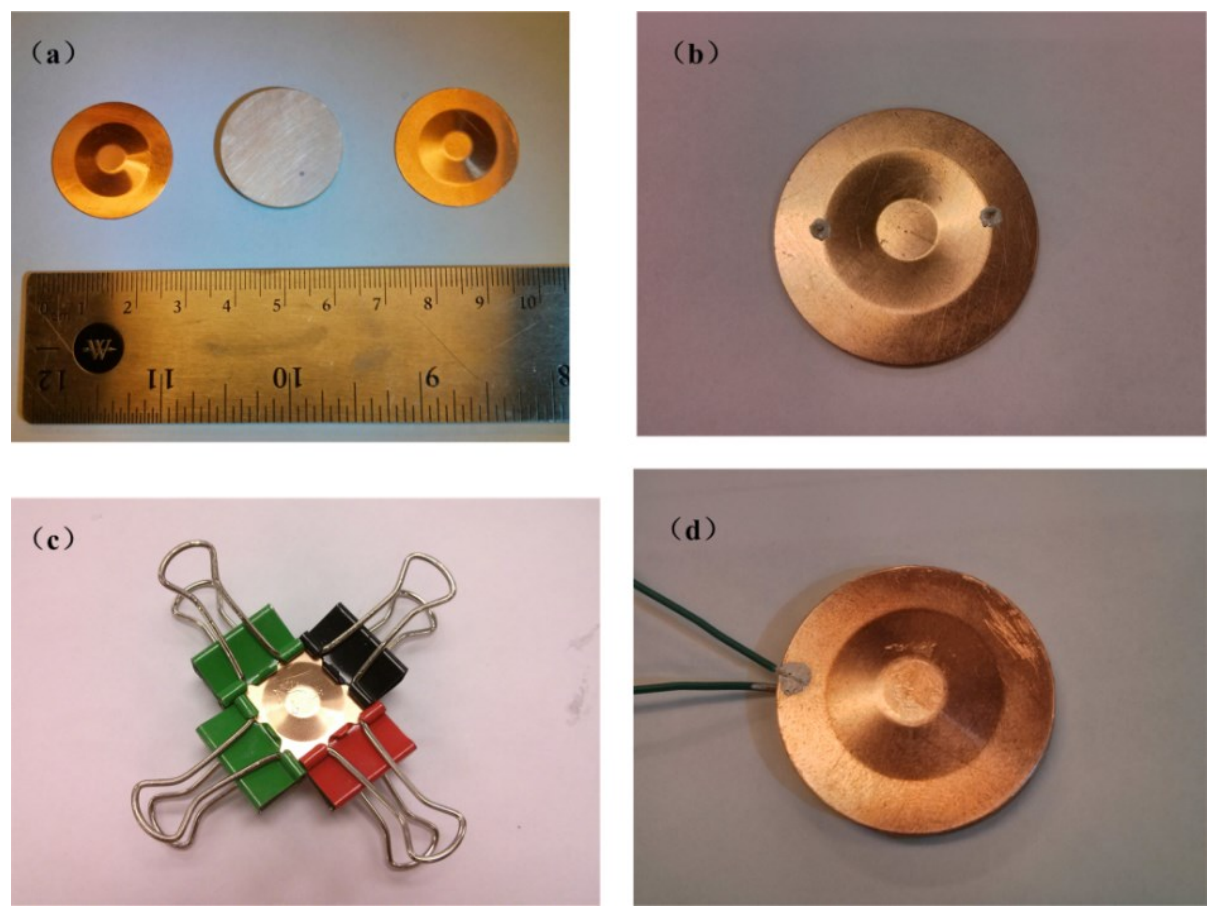

Figure 3.25 Cymbal bonding process.

\subsection{Cymbal Energy Transducer Model and Test}

This section describes the piezoelectric source circuit model and the open circuit voltage test circuit model, from which the total generated charge in each load cycle can be calculated. The open circuit voltage tests are done on the single Cymbal transducer, the Cymbal array container, and the speed bump harvester embedded with Cymbal array.

\subsubsection{Electric circuit model analysis}

\subsubsection{Electric circuit model of piezoelectric source}

Piezoelectric generators can be modeled electrically as a current source $I_{p}(t)$ in parallel with its internal leakage equivalent resistance $R_{p}$ and internal electrode capacitor $C_{p}[59$, 
60], as shown in Figure 3.26. Assuming the internal resistance of piezoelectric unit is large enough to be ignored, the piezoelectric source model can then be simplified to a current source $I_{p}(t)$ and an internal capacitor $C_{p}$. The capacitance of the piezoelectric disk ( $r=12.7 \mathrm{~mm}, h=2 \mathrm{~mm}$ ) used in the Cymbal transducer can be calculated by the capacitance formula of a parallel-plate capacitor:

$$
\begin{gathered}
\mathrm{C}_{\mathrm{p}}=\frac{\varepsilon_{33}^{T} \pi r^{2}}{h}=\frac{\pi\left(3300 \times 8.85 \times 10^{-12}\right)\left(12.7 \times 10^{-3}\right)^{2}}{2 \times 10^{-3}} \\
=7.3992 \mathrm{nF} \approx 7.4 \mathrm{nF}
\end{gathered}
$$

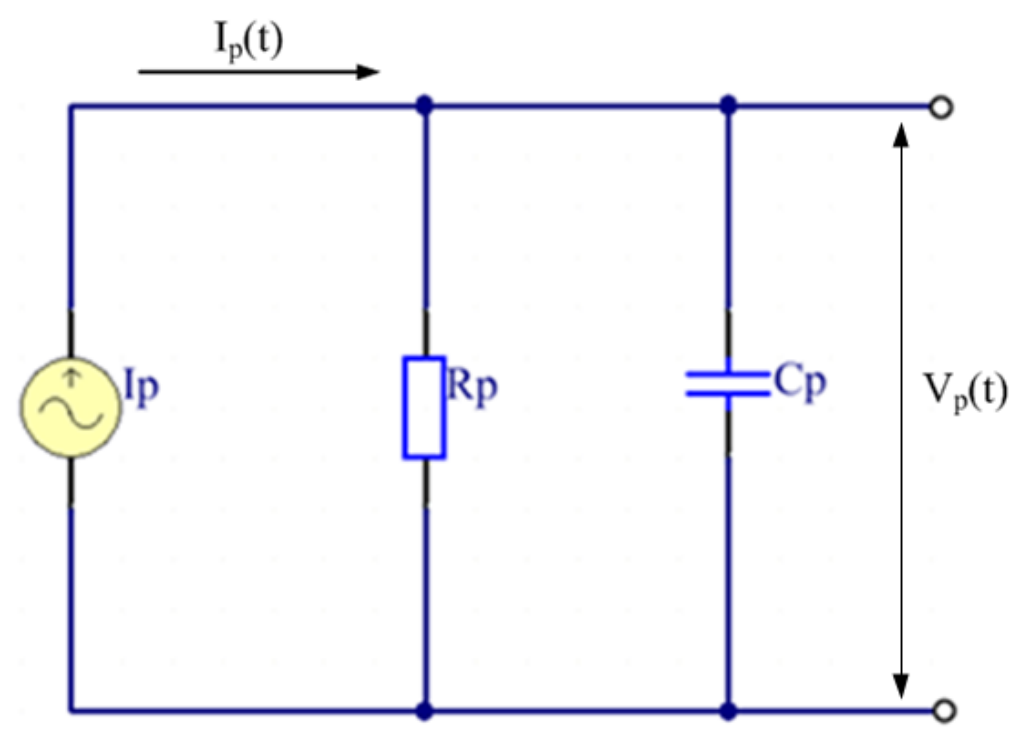

Figure 3.26 Equivalent electric circuit model of Cymbal transducer.

Given that the compression is in the positive displacement direction, the displacement of the Cymbal transducer is always positive under force cycles (compression and release). It is assumed that the mechanical displacement $u(t)$ of the piezoelectric unit is a sinusoid with peak-to-peak value $u_{\max }$ and angular frequency $\omega$ as shown in the first waveform in Figure 3.27. The Cymbal unit is pre-stressed to $u_{m i d}$ and then deforms between 0 and 
$u_{\max }$. The velocity, $v(t)$, is the derivative of the displacement, $u(t)$. Because the open circuit voltage, $V(t)$, is proportional to the mechanical displacement, $u(t), V(t)$ has a similar waveform to $u(t)$ with peak-to-peak amplitude, $V_{p_{-} p e a k}$, and angular frequency, $\omega$. Assuming $V(0)=0$, the open circuit voltage is:

$$
V(t)=\frac{1}{2} V_{p_{-} p e a k}(1-\cos (\omega t))
$$

The waveform of the open circuit voltage is shown in Figure 3.27. The generated piezoelectric current, $I_{p}(t)$, can be derived from the open circuit voltage:

$$
\begin{aligned}
I_{p}(t)=C_{p} \frac{d V}{d t} & =\frac{1}{2} C_{p} V_{p_{-} \text {peak }} \omega \sin (\omega t) \\
& =I_{p} \sin (\omega t)
\end{aligned}
$$

From Equation (3.31), the current source, $I_{p}(t)$, is a sinusoid with amplitude, $I_{p}$, and angular frequency, $\omega$, and it has similar waveform to that of the velocity, $v(t)$, (shown in Figure 3.27). It should be noted that the piezoelectric current peak, $I_{p}$, is calculated assuming the load is capacitive. In open circuit condition, the load is the piezoelectric capacitor. The peak value of the output open-circuit voltage, $V_{p_{-} \text {peak }}$, can be represented by Equation (3.32):

$$
V_{p_{-} \text {peak }}=\frac{2 I_{p}}{\omega C_{p}}
$$

As the generated charge, $Q$, under every full compression of the same displacement is a constant, $V_{p_{-} \text {peak }}$ is also a constant and can be represented as:

$$
V_{p \_p e a k}=\frac{Q}{C_{p}}
$$


By combining Equation (3.32) and (3.33), the amplitude, $I_{p}$, and the angular frequency, $\omega$, is related as:

$$
I_{p}=\frac{Q \omega}{2}
$$

Equation (3.34) indicates that the amplitude of the current, $I_{p}(t)$, is a variable dependent on the angular frequency $\omega$ of the mechanical displacement.

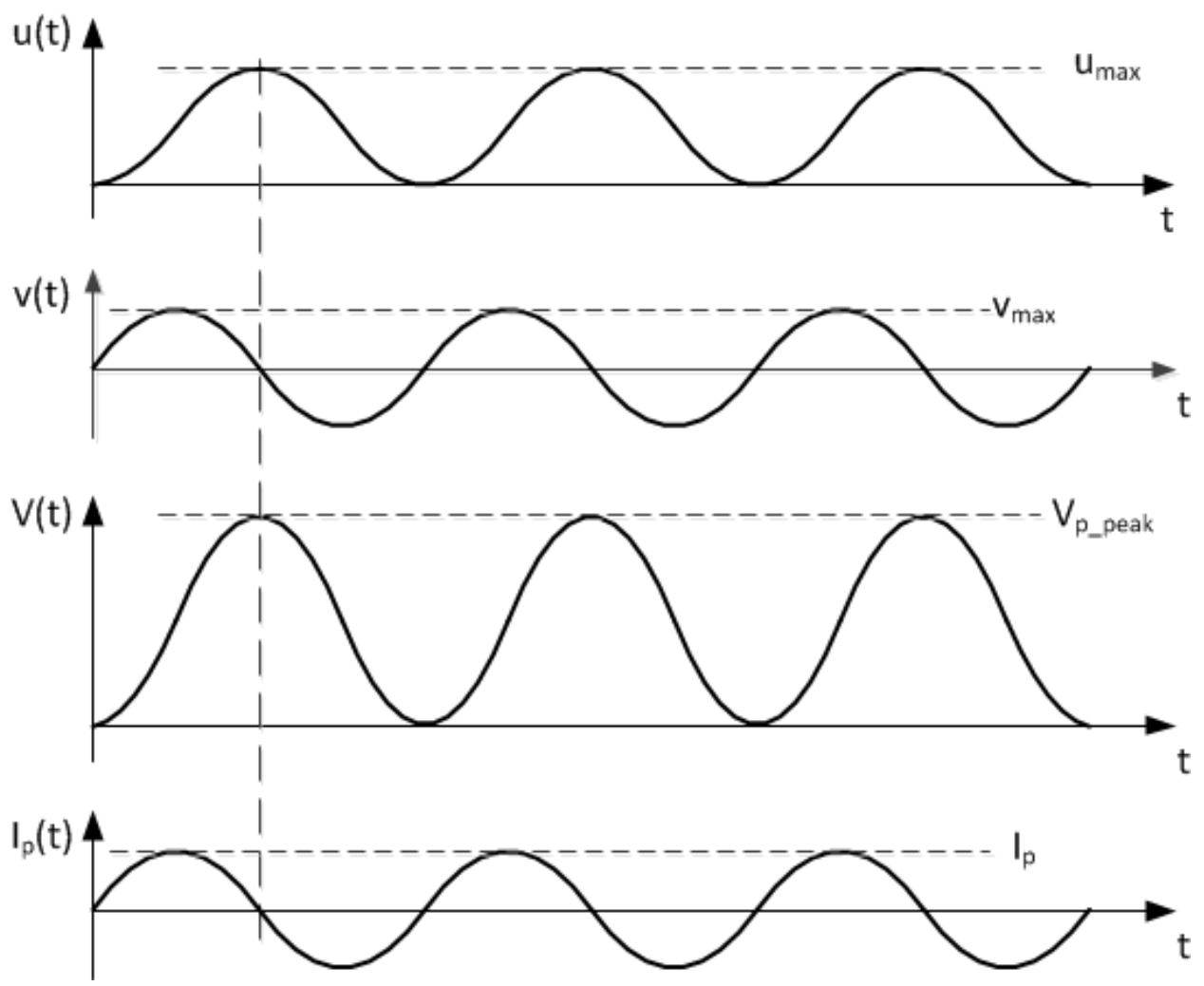

Figure 3.27 Waveforms of Cymbal displacement $u(t)$, velocity $v(t)$, open circuit voltage $V(t)$, and generated current $I_{p}(t)$. 


\subsubsection{Electric circuit model of the open circuit test}

From Equation (3.32) and (3.33), the generated current, $I_{p}$, and charge, $Q$, can be calculated if the open circuit voltage peak, $V_{p_{-} \text {peak }}$, is known. The open circuit voltage of the single Cymbal or the Cymbal array can be readily measured by an oscilloscope while considering the effects of oscilloscope probe. For the oscilloscope (Tektronix ${ }^{\mathrm{TM}}$ DPO 2024B) used in the tests, the input resistance is $10 \mathrm{M} \Omega$ and the input capacitance is less than $4 \mathrm{pF}$ according to its manual. The input capacitance can be ignored because it is relatively much smaller than the PZT disk internal capacitance, $C_{p}$, and its effect on the total capacitance $\left(C=C_{b}+C_{p}\right)$ is relatively small. The input resistance, however, should not be ignored as it is relatively much smaller than the piezoelectric internal resistance, and its effect to the total resistance $\left(R=\frac{R_{p} R_{I n}}{R_{p}+R_{I n}}\right)$ is not negligible.

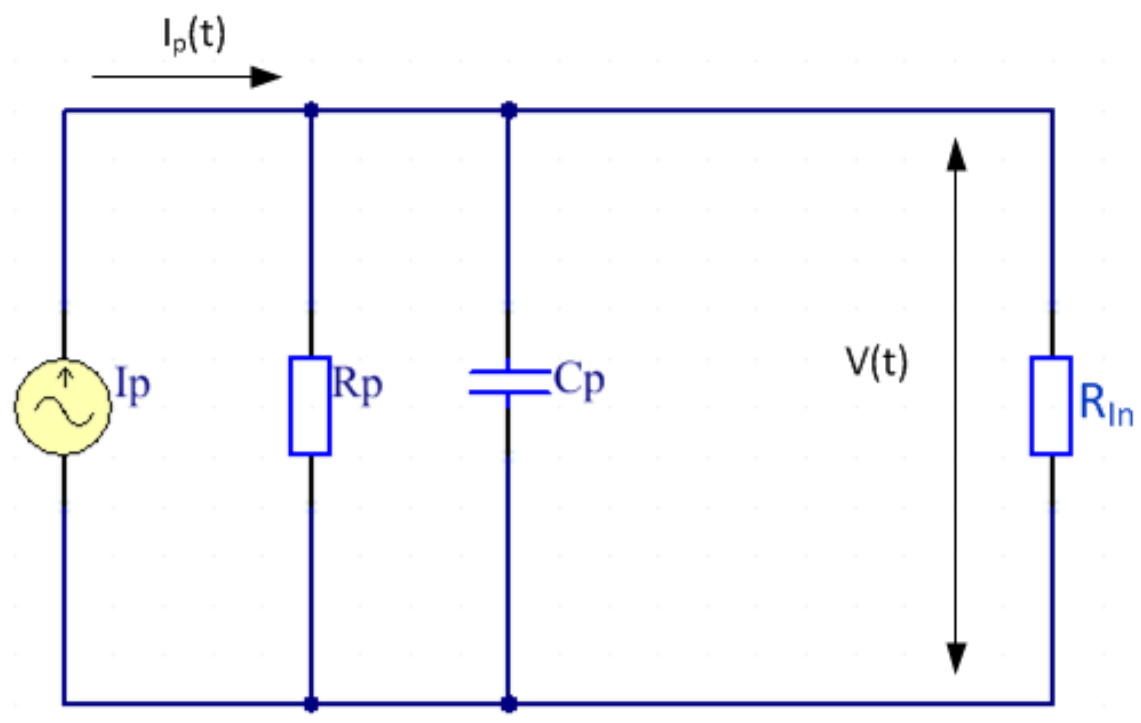

Figure 3.28 Equivalent circuit of open circuit voltage test. 
Figure 3.28 shows the equivalent circuit of the open circuit voltage test considering the input impedance across the oscilloscope probe. The oscilloscope input resistance, $R_{I n}$, and internal resistance, $R_{p}$, can be verified by an RC circuit voltage decay test. $R$ represents the total resistance of $R_{p}$ and $R_{I n}$ in parallel, and $C$ represents the piezoelectric internal capacitor, $C_{p}$, which is calculated as $7.4 \mathrm{nF}$ in Equation (3.29).

The probe resistance can be verified by the following test. Firstly, the Cymbal unit is compressed by a static constant force to keep the charges in $C_{p}$. Secondly, the probe is connected to measure the voltage decay waveform. When the voltage becomes 0 , the probe is removed and $R_{I n}$ is unloaded. After that, the constant force is released and the probe is connected again to measure the voltage decaying through the probe load. Figure 3.29 shows the waveforms of the voltage decay of the compression and load release process, and their corresponding exponential decays are estimated. For an RC circuit with the capacitor initially charged, the voltage across the capacitor is given by:

$$
V=A e^{-t / R C}
$$

In Figure 3.29, the first voltage decay equation is for the compressed Cymbal unit with $\frac{1}{R C}=12.81$, and $R=10.55 \mathrm{M} \Omega$. The second voltage decay is for the released Cymbal unit with $\frac{1}{R C}=13.1$, and $R=10.3 \mathrm{M} \Omega$. The difference is due to the decrease in $C_{P}$ when the Cymbal is compressed, according to Equation (3.29). In test, it is found that the voltage decaying through $R_{I n}$ is much slower than the voltage decaying through $R_{p}$, as the voltage hardly changes without the oscilloscope in the loop. Hence, it can be assumed that $R_{I n}=\frac{10.3+10.55}{2} \approx 10.4 \mathrm{M} \Omega$ and $R_{p}$ is much larger than $R_{I n}$. 


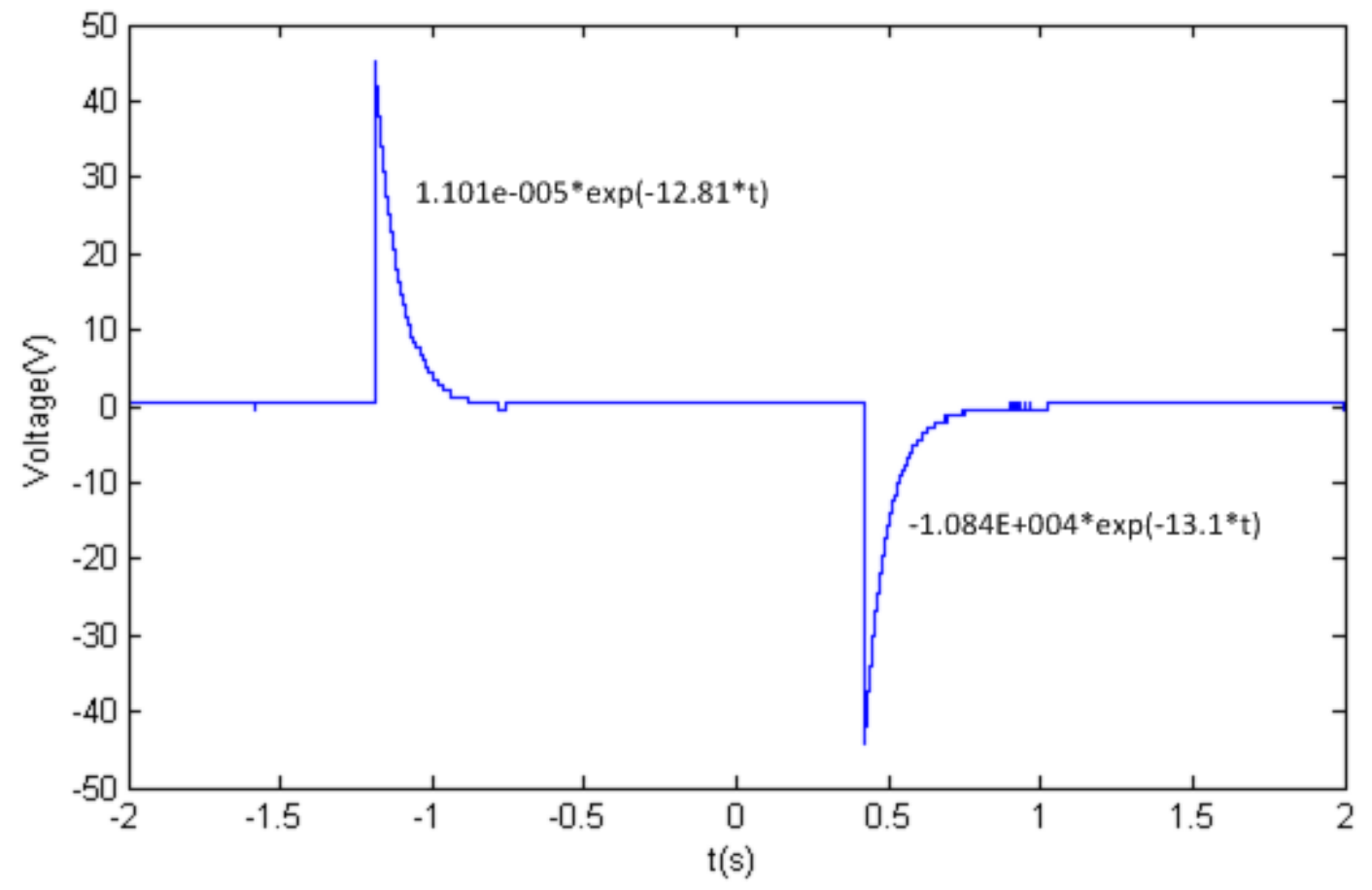

Figure 3.29 Voltage decaying due to the oscilloscope probe.

Assuming that $V(0)=0$ and $I(t)=I_{p} \cos (\omega t)$. Using Kirchhoff current law,

$$
I_{P} \sin (\omega t)=C_{p} \frac{d V}{d t}+\frac{V}{R}
$$

Applying Laplace transform to the above equation yields:

$$
\begin{aligned}
& I_{p} \cdot \frac{\omega}{s^{2}+\omega^{2}}=C_{p} \cdot(s V(s)-V(0))+\frac{V(s)}{R} \\
& V(s)=\frac{I_{p}}{C_{p}} \cdot \frac{\omega}{\left(s^{2}+\omega^{2}\right)\left(s+\frac{1}{R C_{p}}\right)}+\frac{\mathrm{V}(0) \cdot \mathrm{C}_{\mathrm{p}}}{C_{p} s+\frac{1}{R}}
\end{aligned}
$$

By applying Inverse Laplace transform, the output voltage of the PZT can be calculated as: 


$$
\begin{gathered}
V(t)=\frac{I_{p}}{C_{p}}\left(\frac{R C_{p}}{\omega^{2} R^{2} C_{p}^{2}+1} \sin (\omega t)-\frac{R^{2} C_{p}^{2} \omega}{\omega^{2} R^{2} C_{p}^{2}+1} \cos (\omega t)\right. \\
\left.+\frac{R^{2} C_{p}^{2} \omega}{\omega^{2} R^{2} C_{p}^{2}+1} e^{-\frac{t}{R C_{p}}}\right)+V(0) \cdot e^{-\frac{t}{R C_{p}}}
\end{gathered}
$$

Given the waveform of the generated voltage measured by an oscilloscope, the current peak, $I_{p}$, can be calculated using Equation (3.36).

\subsubsection{Single Cymbal open circuit test}

The Cymbal transducer is placed inside a hole of the small nylon plastic container, and the depth of the hole is $4.05 \mathrm{~mm}$, which is $0.15 \mathrm{~mm}$ lower than the Cymbal's thickness (4.2 mm) (Figure 3.30). This container is then placed on a steel protection case bolted on the platform of the MTS load frame.

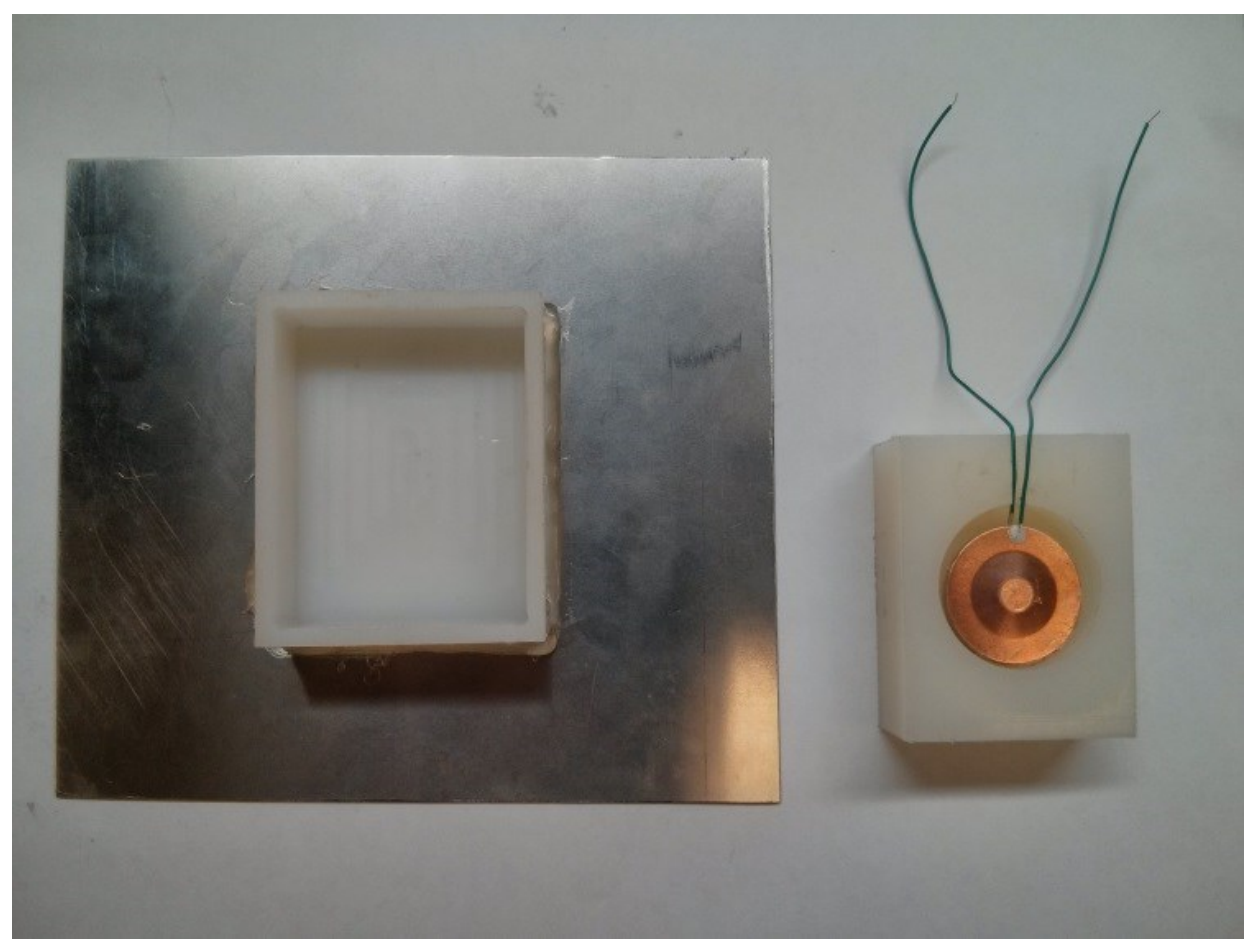

Figure 3.30 Single Cymbal test. 
This single Cymbal is tested under a hydraulic load frame by a sinusoidal compression with $180 \mathrm{~N}$ amplitude at $2 \mathrm{~Hz}$. The output open circuit voltage is measured by the oscilloscope (Model: Tektronix ${ }^{\mathrm{TM}}$ DPO 2024B), and the data are recorded as Excel files by the oscilloscope data acquisition software "OPENCHOICE DESKTOP". Figure 3.31 shows that the open circuit voltage output of the Cymbal unit is measured approximately $120 \mathrm{~V}$.

Substituting $\omega=4 \pi, R=10.4 \mathrm{M} \Omega, C_{p}=7.4 \mathrm{nF}$ into Equation (3.36), the piezoelectric voltage is:

$$
\begin{aligned}
V(t)= & \frac{I_{p}}{7.4 \times 10^{-9}}\left(0.039 \sin (4 \pi t)-0.0477 \cos (4 \pi t)+0.0477 e^{-\frac{t}{0.074}}\right) \\
= & \frac{I_{p}}{7.4 \times 10^{-9}}\left(0.0616 \sin (4 \pi t-0.8854)-0.0477 e^{-\frac{t}{0.074}}\right)
\end{aligned}
$$

If the decaying time is long enough for the exponential item to be ignored, the amplitude of $V(t)$ becomes $\frac{0.0616 I_{p}}{7.4 \times 10^{-9}}=8.324 \times 10^{6} I_{p}$, which is measured at $120 \mathrm{~V}$. The amplitude of the current under $2 \mathrm{HZ}$ mechanical loading is:

$$
I_{p}=\frac{120}{8.324} \times 10^{-6}=14.416 \mu \mathrm{A}
$$

The charge generated during each compression is calculated from Equation (3.34),

$$
Q=\frac{2 I_{p}}{\omega}=\frac{2 \times 14.416 \times 10^{-6}}{4 \pi}=2.294 \times 10^{-6} \mathrm{C}
$$

Therefore, the amplitude of the open circuit voltage without the effects of oscilloscope probe impedance can then be estimated from Equation (3.33): 


$$
V_{p_{-} \text {peak }}=\frac{Q}{C_{p}}=\frac{2.294 \times 10^{-6}}{7.4 \times 10^{-9}}=310 \mathrm{~V}
$$

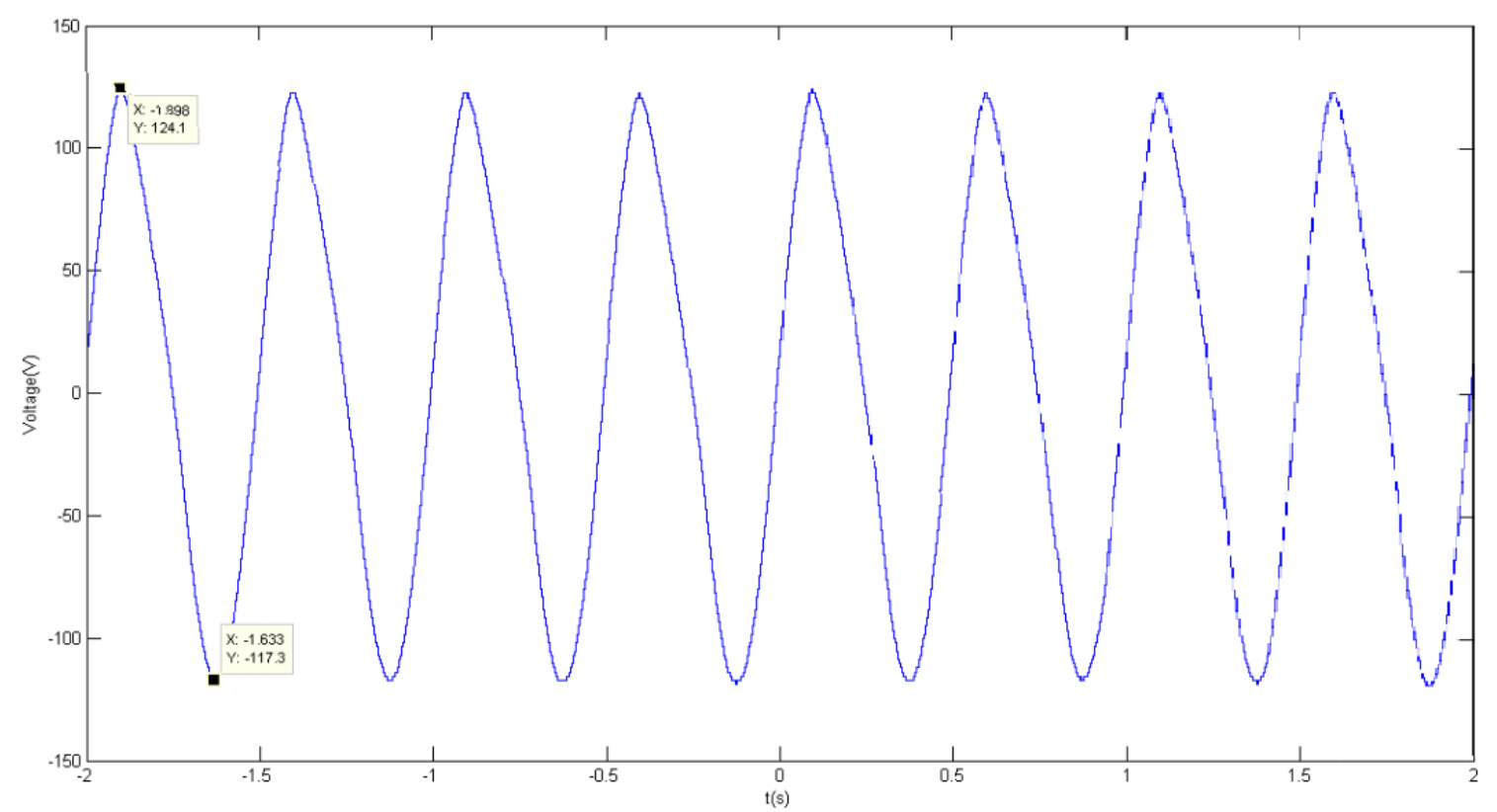

Figure 3.31 Open circuit voltage of Cymbal unit.

\subsubsection{Cymbal array design and test}

Cymbal array makes use of multiple Cymbals to increase the energy harvested, and has been used for piezoelectric sound projector and hydrophone [66]. The multi-stacked Cymbal transducer is a stack of Cymbals that is connected in parallel, and has been used on Cymbal actuators for more displacement amplification [67].

In the case of using the multi-stacked Cymbal array for $\mathrm{KEH}$, the electric connection method of the multi-stacked Cymbals in the Cymbal array needs to be determined. There are three possible connections: (1) All the multi-stacked Cymbals are connected in parallel before connecting to a rectifier; (2) Each multi-stacked Cymbal is connected with 
one rectifier before connecting in parallel; (3) All the multi-stacked Cymbals are connected in series before one rectifier.

Because each Cymbal unit has its own location in a multi-stacked Cymbal array, when the wheel rolls over the road, the loads on the Cymbals may be of different magnitudes and phases. If all the Cymbals are connected directly in parallel before rectification, the AC electrical power from each Cymbal may be counteracted, and therefore the total output energy would be reduced $[68,69,70]$. If the Cymbal array is connected in series, the open circuit voltage generated will be multiplied by the number of the Cymbals in series [69]. Given the fact that each Cymbal has an open circuit voltage of hundreds volts under a load of $180 \mathrm{~N}$, the total open circuit voltage of the Cymbal array could be too high to be regulated. It will be further discussed in the next Chapter that a high open circuit voltage is usually not recommended for the power conditioning circuit. The connection method (2), connecting the Cymbals in parallel after their rectifiers, is therefore adopted as the Cymbal connection method in this work, and it will not only solve the problem of phase difference of each Cymbal, but also keep the open circuit voltage at the desired low level. Although this method will use more rectifiers, it can generate more energy than the other two methods.

The Cymbal array container is made of Cast Nylon from McMaster-CARR ${ }^{\circledR}$ and machined by a $\mathrm{CNC}$ machine. In the container, a $3 \times 3$ Cymbal array with two layers is placed (Figure 3.32). 


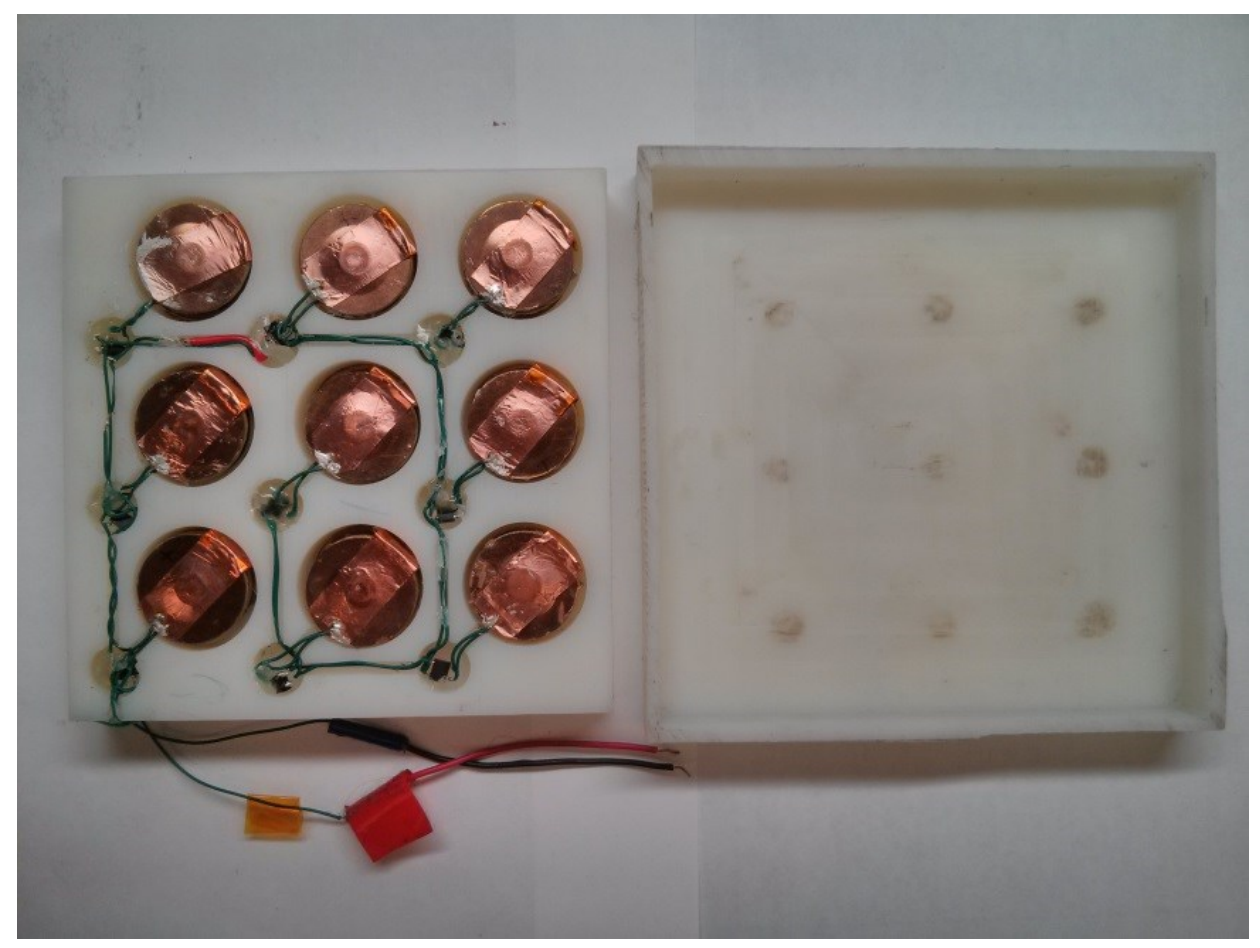

Figure 3.32 Cymbal array layout.

The larger cavities in the container are for cymbals, and the smaller cavities are for the rectifiers. Slots are cut between these cavities for electric wires. The larger cavities' depth is $8.1 \mathrm{~mm}$, which is $0.3 \mathrm{~mm}$ shorter than the stack of two Cymbals $(4.2 \mathrm{~mm} \times 2=$ 8.4 mm). The detailed container drawing is shown in Figure B.1 in Appendix B. In each cavity, the two Cymbals are connected in parallel with conductive bronze tape. This Cymbal array is designed to work safely under $1600 \mathrm{~N}$. In the case that the load is over $1600 \mathrm{~N}$, the nylon between the Cymbals will endure the extra load to protect the Cymbals from over-loading damage.

The Cymbal array harvester is tested under a loading from a hydraulic load frame, and the loading has a peak-to-peak force amplitude of $1600 \mathrm{~N}$ with a frequency of $1 \mathrm{~Hz}$. The 
setup of the test is shown in Figure 3.33, and the output piezoelectric voltage is measured by the oscilloscope (Model: Tektronix ${ }^{\mathrm{TM}}$ DPO 2024B).

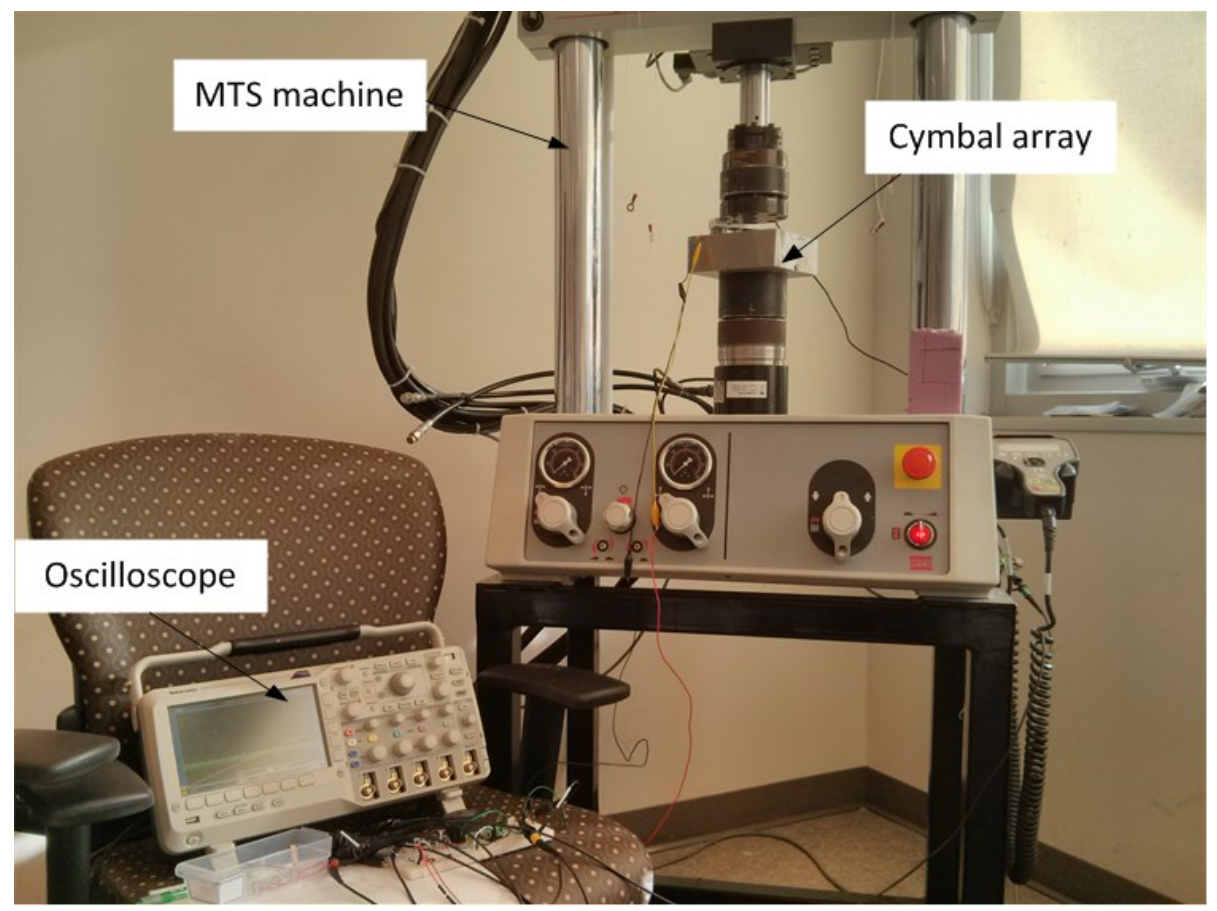

Figure 3.33 Cymbal array test setup.

Figure 3.34 illustrates the open circuit voltage output of the Cymbal array under the loading of the load frame. It is noted that the voltage peaks for compression and load release are different; this is because the cyclic load is a sinusoid from being released (point A) to being compressed (point B), and then to being released (point C). Before the next load, the load is zero from point $\mathrm{C}$ to point $\mathrm{D}$, and the voltage keeps draining during this time. Therefore, it is assumed that the output voltage is harmonic from the high peak $(142 \mathrm{~V},-1.55 \mathrm{~s})$ to the lower peak $(70 \mathrm{~V},-1.20 \mathrm{~s})$, and therefore the harmonic voltage has a cycle $T=2 \times(1.55 \mathrm{~s}-1.20 \mathrm{~s})=0.7 \mathrm{~s}$, and the frequency is $f=\frac{1}{T}=\frac{10}{7} \mathrm{~Hz}$. 
Therefore, the compression starts at $26 \mathrm{~V}$ at $-1.9 \mathrm{~s}$, which is $0.35 \mathrm{~s}$ (i.e., half of the cycle $T$ ) earlier than the high peak.

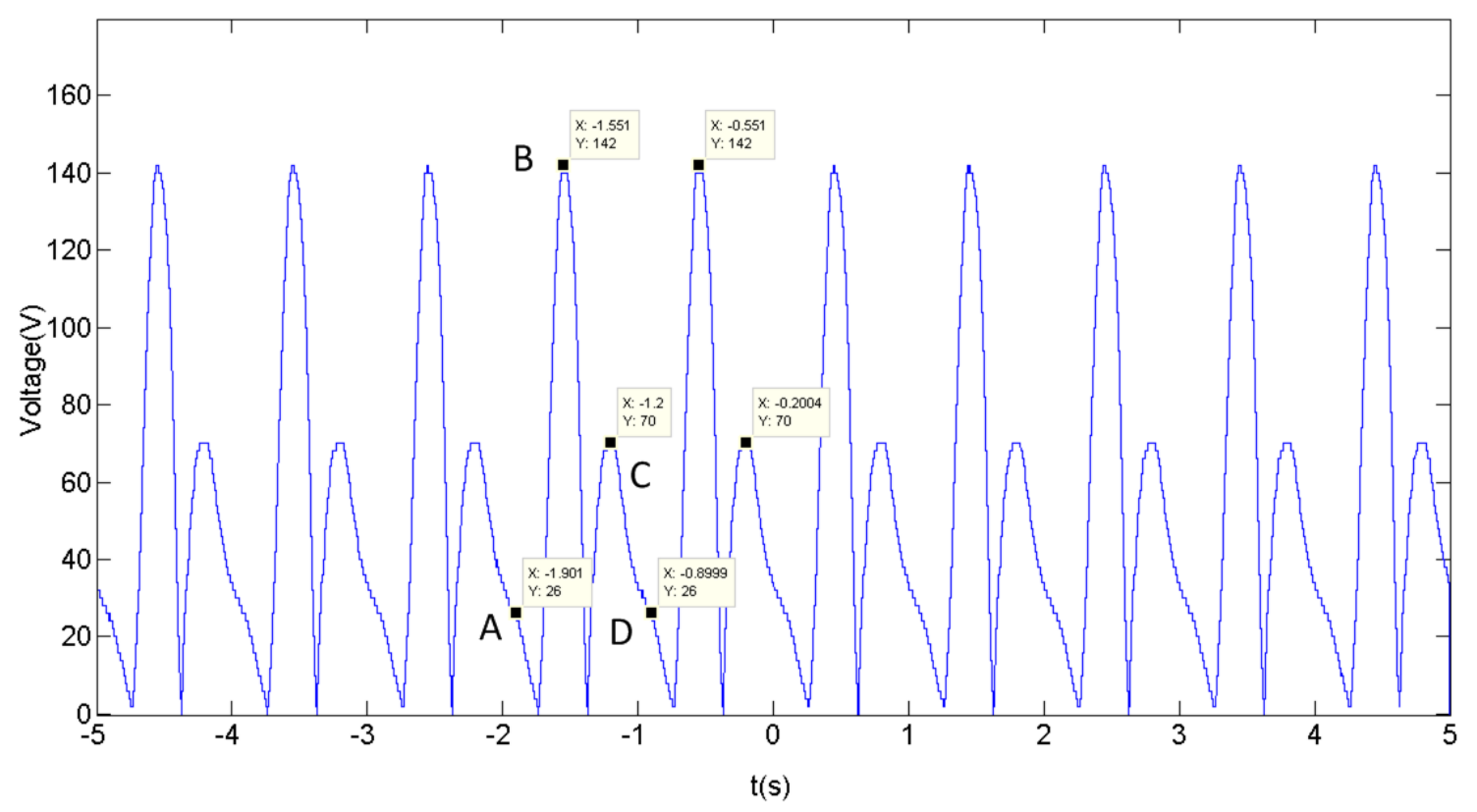

Figure 3.34 Open circuit Voltage output of Cymbal array.

Substituting $\omega=2 \pi f=\frac{20}{7} \pi, C_{p}=18 \times 7.4 \mathrm{nF}=133.2 \mathrm{nF}$, and $V(0)=-48 \mathrm{~V}$ into Equation (3.36), the piezoelectric voltage is:

$$
\begin{gathered}
V(t)=\frac{I_{p}}{133.2 \times 10^{-9}}\left(0.0089 \sin \left(\frac{20}{7} \pi t\right)-0.1107 \cos \left(\frac{20}{7} \pi t\right)\right. \\
\left.+0.1107 e^{-\frac{t}{R C_{P}}}\right)-26 \cdot e^{-\frac{t}{R C_{p}}} \\
=\frac{I_{p}}{133.2 \times 10^{-9}}\left(0.1107 \sin \left(\frac{20}{7} \pi t-1.4906\right)+0.1107 e^{-\frac{t}{R C_{p}}}\right) \\
-26 \cdot e^{-\frac{t}{R C_{p}}}
\end{gathered}
$$

Substituting $V(0.35 \mathrm{~s})=142 \mathrm{~V}$, and $V(0.7 s)=70 \mathrm{~V}$ into Equation (3.37) yields: 


$$
\left\{\begin{array}{c}
R=5.2 \mathrm{M} \Omega \\
I_{p}=0.119 \mathrm{~mA}
\end{array}\right.
$$

The resistant $(R=5.2 \mathrm{M} \Omega)$ is smaller than the oscilloscope internal resistant $\left(R_{I n}=\right.$ 10.4 $\mathrm{M} \Omega$ ) because of the resistance of the full wave rectifiers. From Equation (3.34), the charge generated during each compression is:

$$
Q=\frac{2 I_{p}}{\omega}=\frac{2 \times 119 \times 10^{-6}}{\frac{20}{7} \pi}=2.652 \times 10^{-5} \mathrm{C}
$$

The amplitude of the open circuit voltage without the effects of the impedance of the oscilloscope probe can be estimated by Equation (3.33):

$$
V_{p_{\text {_peak }}}=\frac{Q}{C_{p}}=\frac{2.652 \times 10^{-5}}{7.4 \times 18 \times 10^{-9}}=199 \mathrm{~V}
$$

Compared to the single Cymbal open circuit test, the output voltage is smaller. This is because each Cymbal in the Cymbal array experiences different loading forces due to the variations in their positions and fabrication tolerances.

\subsubsection{Energy harvesting speed bump design and test}

In order to test the Cymbal array under vehicles, an off-the-shelf speed bump (Barco Mini Speed Hump) embedded with a rectangular Cymbal container is made, and this container is designed to hold a $2 \times 5$ Cymbal array with two layers inside (as illustrated in Figure 3.35). 


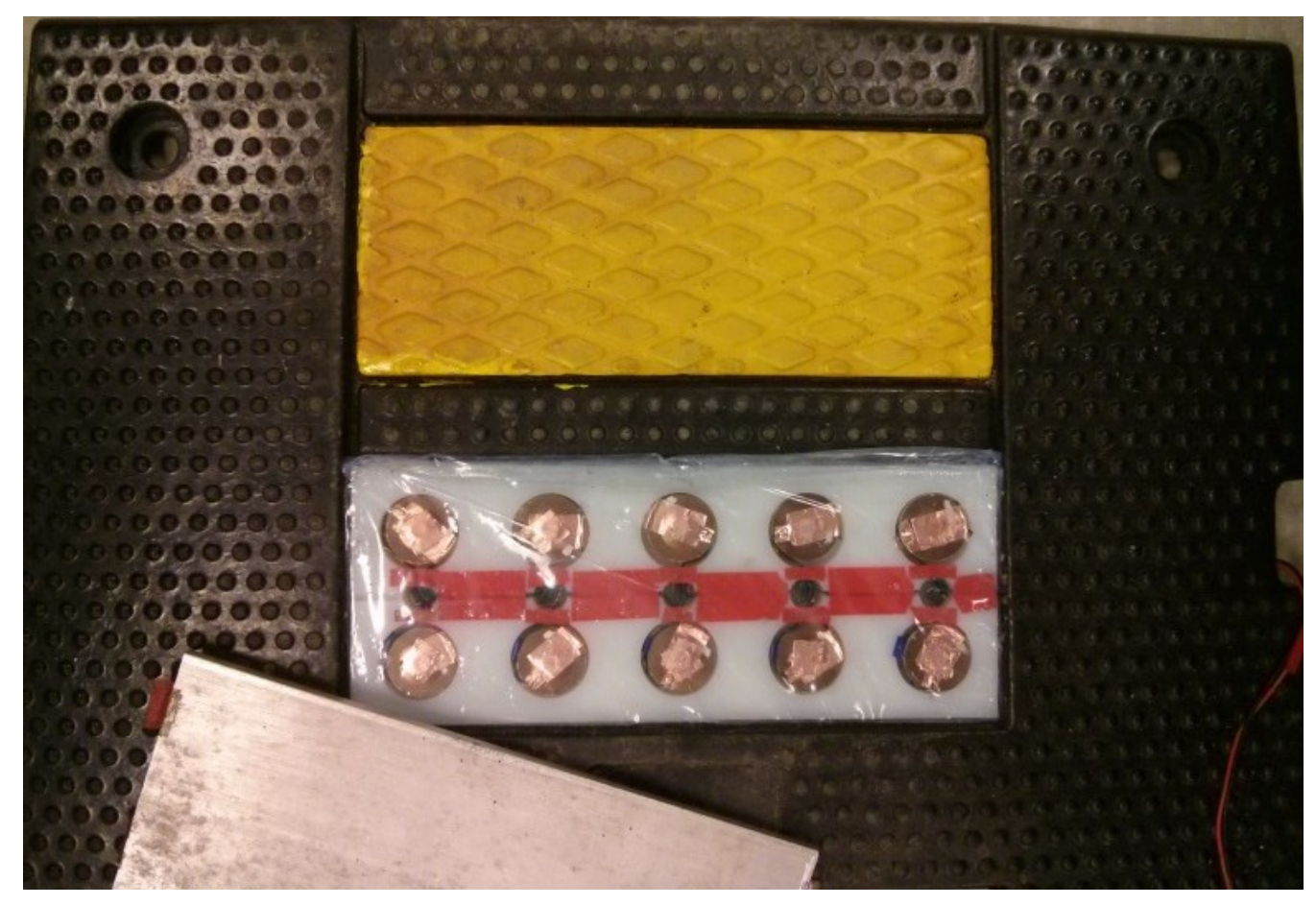

Figure 3.35 Speed bump embedded with Cymbal array.

The $2 \times 5$ Cymbal array is able to endure $1800 \mathrm{~N}$ itself, and if the load is over that value, the nylon between the Cymbals will hold the extra load to protect the Cymbals from over-loading damage. The detailed dimensions of the container are shown in Figure B.2 in Appendix B.

The open circuit voltage test setup of the speed bump energy harvester is shown in Figure 3.36; the energy harvesting circuit is not utilized here because the harvester is connected directed to the oscilloscope. The oscilloscope records the open circuit voltage waveform when the front wheel of the test vehicle (2003 Subaru Outback) rolls over the speed bump back and forth. 


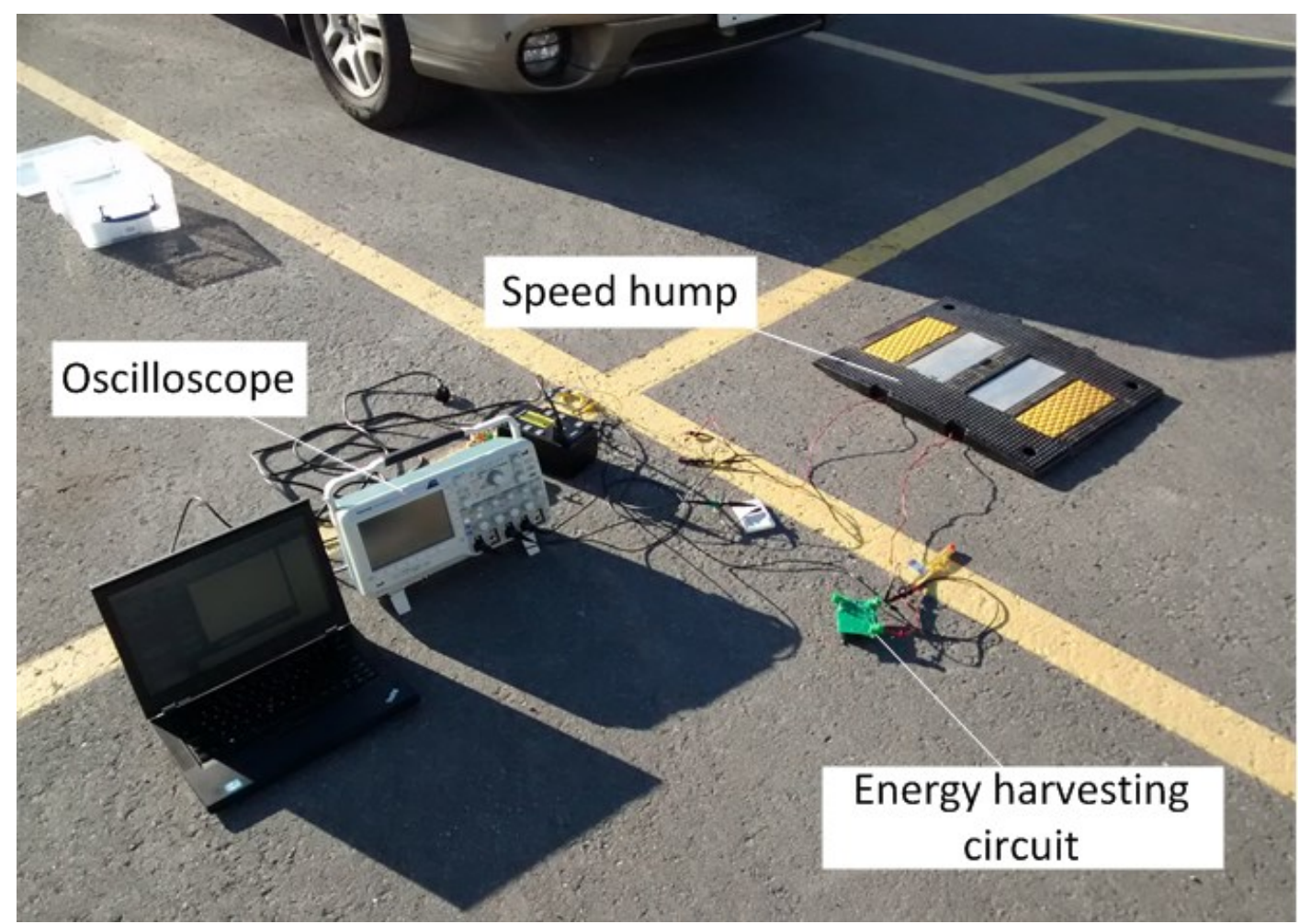

Figure 3.36 Speed bump energy harvester test setup.

As shown in Figure 3.37, the open circuit voltage peaks are generated by the front wheel during the test. From point $\mathrm{A}$ to point $\mathrm{B}$, the open circuit voltage increases from $2 \mathrm{~V}$ to $228 \mathrm{~V}$ in $0.028 \mathrm{~s}$, which is also half of the load cycle $T$. Therefore, $T$ equals $0.056 \mathrm{~s}$. 


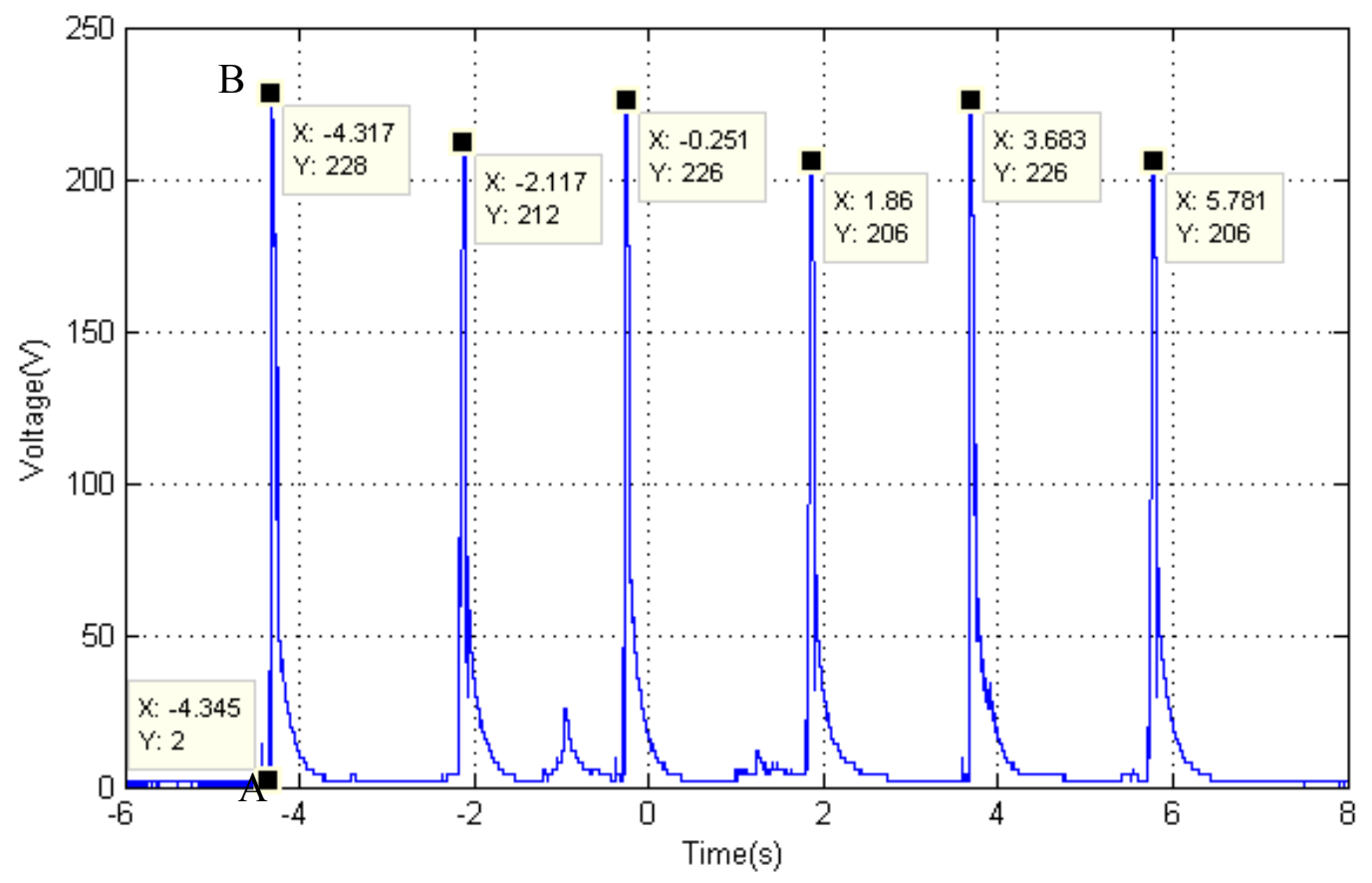

Figure 3.37 Open circuit voltage of the speed bump harvester.

Substituting $\omega=\frac{2 \pi}{T}=35.17 \pi, \mathrm{R}=5.2 \mathrm{M} \Omega, C_{p}=20 \times 7.4 \mathrm{nF}=148 \mathrm{nF}$ into Equation (3.36), the piezoelectric voltage is:

$$
\begin{gathered}
V(t)=\frac{I_{p}}{1.48 \times 10^{-7}}(0.000106 \sin (35.17 \pi t)-0.009 \cos (35.17 \pi t) \\
\left.+0.009 e^{-\frac{t}{0.77}}\right)
\end{gathered}
$$

Because the time constant $R C_{p}$ is significantly larger than the period $\mathrm{T}$, the exponential item changes little. The voltage peak should be:

$$
\begin{gathered}
V(0.028)=\frac{I_{p}}{1.48 \times 10^{-7}}(0+0.009 \times 1+0.009 \times 0.964)=1.194 \times 10^{5} I_{p} \\
=228 \mathrm{~V}
\end{gathered}
$$

The amplitude of the current under this mechanical load is: 


$$
I_{p}=\frac{228}{1.194} \times 10^{-5}=1.91 \mathrm{~mA}
$$

The charge generated during the compression of the front wheel is calculated from Equation (3.34),

$$
Q=\frac{2 I_{p}}{\omega}=\frac{2 \times 1.91 \times 10^{-3}}{35.17 \pi}=3.457 \times 10^{-5} \mathrm{C}
$$

The amplitude of the open circuit voltage without considering the effects of dissipation can then be estimated from Equation (3.33):

$$
V_{p_{-} \text {peak }}=\frac{Q}{C_{p}}=\frac{3.457 \times 10^{-5}}{1.48 \times 10^{-7}} \approx 234 \mathrm{~V}
$$

The calculated open circuit voltage is close to the measured voltage because the force period is extremely short and the effects of dissipation are very limited. 


\section{Chapter: KEH Circuit Topology Determination}

From the piezoelectric equivalent electric circuit and the open circuit voltage tests in the previous chapter, the piezoelectric source is found to be capacitive and generates sinusoidal open circuit voltage of high amplitude under sinusoidal external loading. The voltage generated is $\mathrm{AC}$, but the electronic devices or electrochemical batteries require a stable DC voltage. Therefore the very first stage of the energy harvesting topology of piezoelectric materials is a diode bridge to convert the $\mathrm{AC}$ voltage to $\mathrm{DC}$ voltage. The energy storage unit is chosen to be a capacitor or a supercapacitor depending on the

power requirement of the application, because compared with the Lithium battery, they have much longer lifetime (more than 10 years and 100,000 cycles) and low internal series resistance that allows high charging current $[71,72]$. Because most electronic devices require an input voltage much lower than the piezoelectric open circuit voltage, the next stage is to transfer the energy generated from a small piezoelectric internal capacitor of high open circuit voltage to a large bucket capacitor of much lower rectified voltage.

An electromechanical model of the Cymbal transducer is made, which gives the aim of the energy harvesting topology design. Different piezoelectric KEH circuit topologies have been compared under different load impedance [73]. These topologies' performance under different rectified voltage is worthy of study. Seven recent power harvesting topologies are studied and compared in terms of the bucket capacitor energy increment 
under different rectified voltages, and an optimal power harvesting topology has been chosen for implementation in this project.

\subsection{Electromechanical model}

A single Cymbal transducer placed in a container can be modeled as an electromechanical structure shown in Figure 4.1, which is composed of a rigid cover of mass $M$, a viscous damper $C$, a spring with stiffness $K$ modeling the total stiffness of the Cymbal transducer, and an equivalent piezoelectric block modeling the piezoelectric material in the Cymbal [74]. $F$ represents the external cyclic force on the Cymbal unit, and $u$ is the displacement of the container cover.
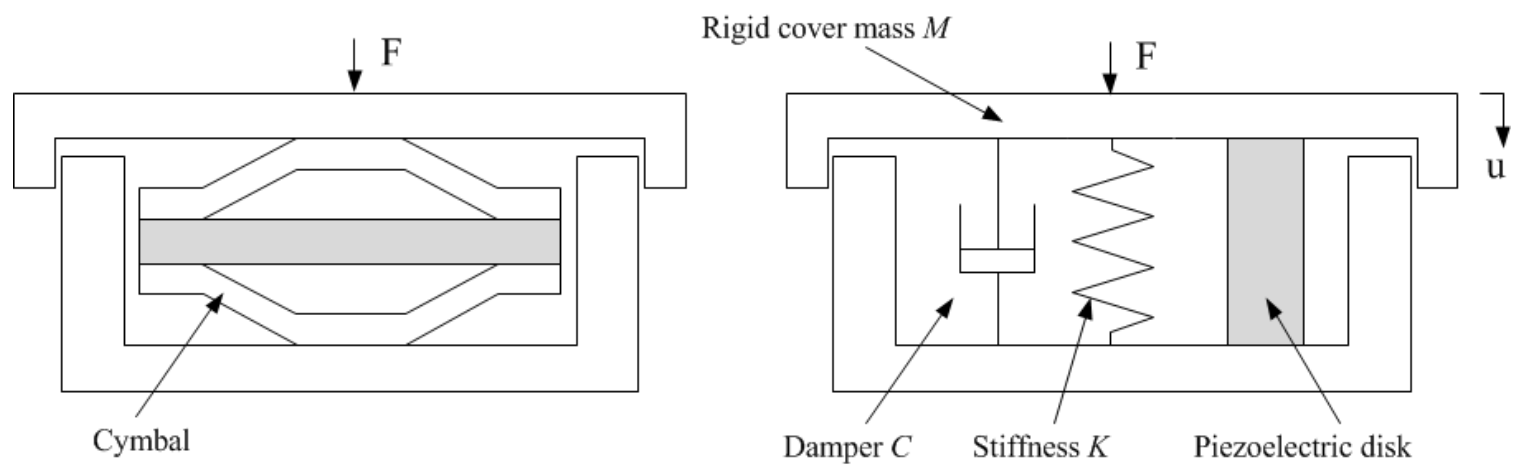

Figure 4.1 Electromechanical model of Cymbal.

The dynamic governing equation of this electromechanical model is:

$$
M \ddot{u}+C \dot{u}+K u+\alpha V_{p}=F
$$

where $V_{p}$ is the piezoelectric voltage, and $\alpha$ is the force factor $\left(\alpha=\frac{F}{V_{p}}\right)$, and therefore $\alpha V_{p}$ is the force generated according to the converse piezoelectric effect. The energetic equation can be derived from Equation (4.1) by integrating both sides with respect to $u$. 


$$
\begin{aligned}
& \int F d u=\int M \ddot{u} d u+\int C \dot{u} d u+\int K u d u+\int \alpha V_{p} d u \\
& \int F \dot{u} d t=\frac{1}{2} M \dot{u}^{2}+C \int \dot{u}^{2} d t+\frac{1}{2} K u^{2}+\alpha \int V_{p} \dot{u} d t
\end{aligned}
$$

Equation (4.3) points out that the energy provided by the external force $F$ is distributed into four components: the kinetic energy of the rigid cover $\left(\frac{1}{2} M \dot{u}^{2}\right)$, the mechanical energy losses of the viscous damper $\left(C \int \dot{u}^{2} d t\right)$, the elastic potential energy of the

Cymbal structure $\left(\frac{1}{2} K u^{2}\right)$, and the converted piezoelectric energy $\left(\alpha \int V_{p} \dot{u} d t\right)$. It should be noted that $\dot{u}(t)$ is preset and has no phase shift with $I_{p}(t)$ from Figure 3.26. For maximizing the converted piezoelectric energy $\left(\alpha \int V_{p} \dot{u} d t\right)$, in the topology selection there are two requirements that should be given priority to: (1) $V_{p}(t)$ and $I_{p}(t)$ should have as small phase shift as possible; (2) $V_{p}(t)$ should be as high as possible.

\subsection{Direct discharge topology}

Direct discharge topology is the simplest power harvesting technique, which is simply connecting the piezoelectric unit with the bucket capacitor through a diode bridge. There is no impedance matching between the energy source and the bucket capacitor. Two types of direct discharge topology are discussed here, the full-bridge direct discharge topology and the half-bridge direct discharge topology. 


\subsubsection{Power output of the direct discharge topology through a full-wave rectifier}

In the direct discharge topology shown in Figure 4.2, the piezoelectric generator is directly connected to a bucket capacitor $C_{b}$ through a full-wave rectifier. It is assumed that $C_{b}$ is much larger than $C_{p}$ and the internal resistance of the piezoelectric unit is high enough to be negligible.

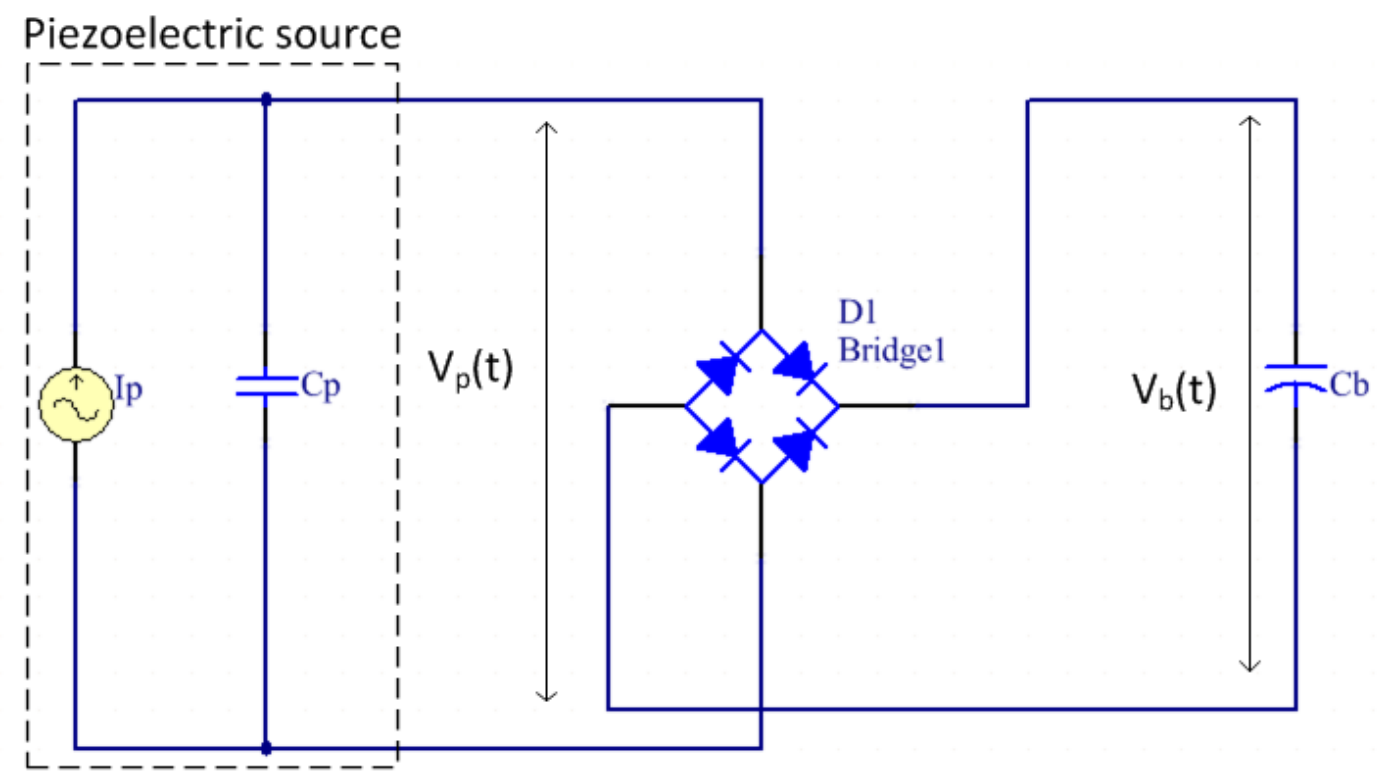

Figure 4.2 Equivalent circuit of the direct discharge topology with full-wave rectifier.

MIT Media laboratory utilizes this topology in the electric circuit of their self-powered RFID (Radio Frequency Identification) sneakers [31]. A bucket capacitor starts discharging current to the RFID when the capacitor is charged to a pre-determined voltage threshold, and the discharging process is disrupted as soon as the voltage drops under a lower voltage threshold. The output power of the full-wave rectifier direct discharging topology is calculated as follows. 
From Equation (3.31), the electric piezoelectric current $I_{p}(t)$ is sinusoidal. $I_{p}$ is the peak value of the output current sinusoid, and $\omega=2 \pi f$ is the angular frequency of the current source. $f$ is the frequency at which the piezoelectric generator is excited, and $T$ represents the period. As shown in Figure 4.3, the charging process of each compression can be separated into two time intervals. In the first interval $0<t<t_{1}$, the piezoelectric voltage $V_{p}$ is below the sum of the voltage of the bucket capacitor $V_{b}$ and the voltage drop $\left(2 V_{d}\right)$ on two of the full-wave rectifier diodes. In this interval, diodes $D_{1}$ are reverse biased. The charge left in the piezoelectric units will be neutralized in this interval at first, before the full bridge diodes are forward biased again. In the second time interval $t_{1}<$ $t<\frac{T}{2}$, the piezoelectric voltage reaches the rectified voltage $\left\langle V_{b}\right\rangle$, and the piezoelectric current starts to flow into both the piezoelectric internal capacitor $C_{p}$ and the bucket capacitor $C_{b}$. After that, during $\frac{T}{2}<t<T$, the loaded force is decreasing, and the piezoelectric current direction is inverted. As for the full-wave rectifier, the charging process during this release process is similar to the compression process, and the generated energy is the same. 


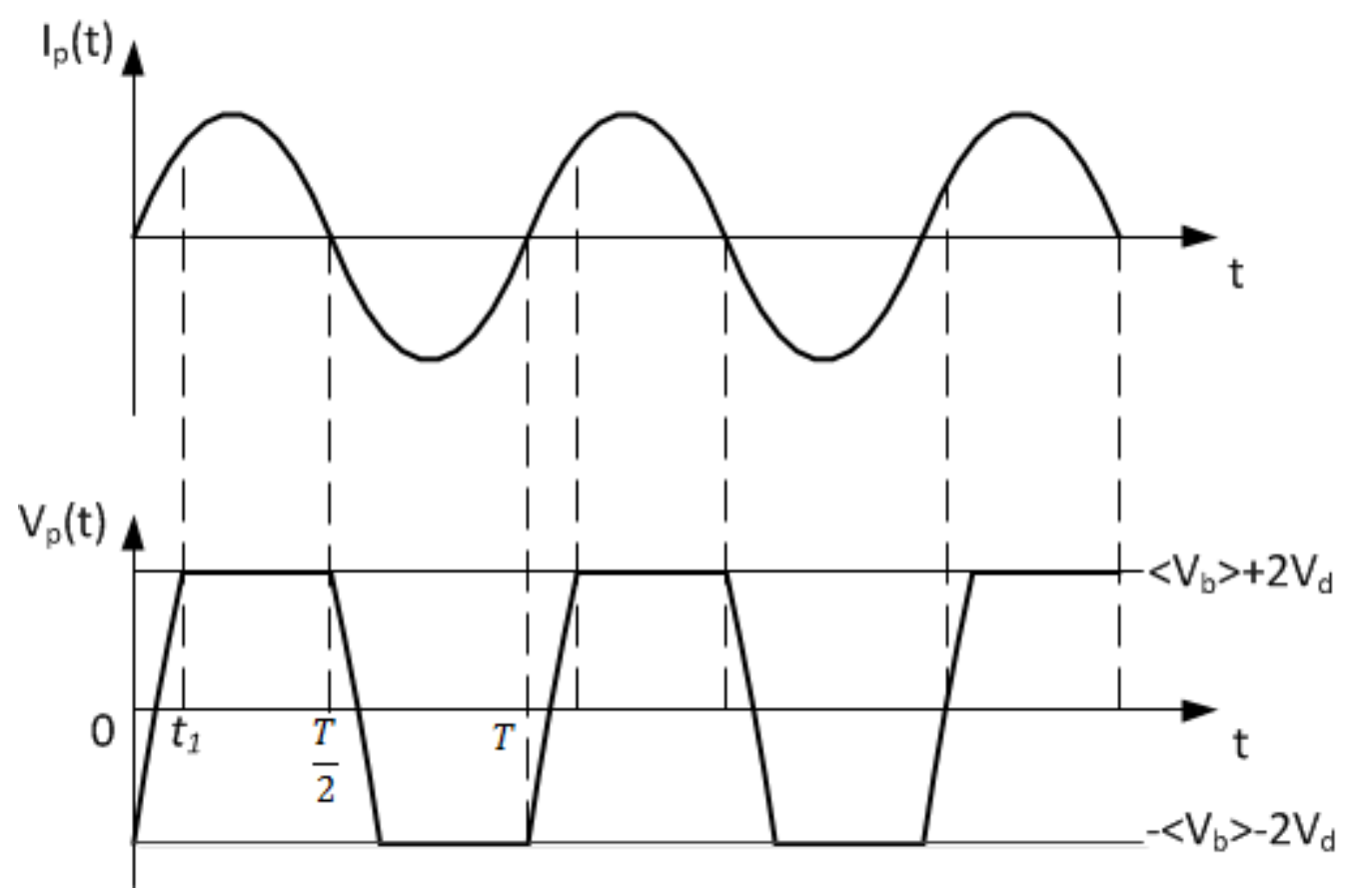

Figure 4.3 Waveforms of piezoelectric current and voltage of direct discharge topology with full-wave rectifier.

Figure 4.3 shows the general waveform of $I_{p}(t)$ and $V_{p}(t)$, the rectified voltage increases under each force cycle in the real case. Given the bucket capacitor voltage just before each compression $\left\langle V_{b}\right\rangle$ and just after each compression $\left\langle V_{b}^{\prime}\right\rangle$, the energy increment of the bucket capacitor during the compression $E_{\text {com }}$ can be calculated as:

$$
E_{\text {com }}=\frac{1}{2} C_{b}\left(\left\langle V_{b}^{\prime}\right\rangle^{2}-\left\langle V_{b}\right\rangle^{2}\right)
$$

$\left\langle V_{b}^{\prime}\right\rangle$ can be calculated by the bucket capacitor net increased charge, which is the total generated piezoelectric charge $Q$ minus the charge wasted on reversing the piezoelectric voltage from $\left(-\left\langle V_{b}\right\rangle-2 V_{d}\right)$ to $\left(\left\langle V_{b}\right\rangle+2 V_{d}\right)$. Assuming $C_{b} \gg C_{p}$,

$$
\left\langle V_{b}^{\prime}\right\rangle=\left\langle V_{b}\right\rangle+\frac{Q-C_{p}\left(2\left\langle V_{b}\right\rangle+4 V_{d}\right)}{\left(\frac{C_{p} C_{b}}{C_{p}+C_{b}}\right)}
$$




$$
=\left\langle V_{b}\right\rangle+\frac{Q-C_{p}\left(2\left\langle V_{b}\right\rangle+4 V_{d}\right)}{C_{b}}
$$

Combining (4.4) and (4.5),

$$
E_{\text {com }}=\frac{1}{2} C_{b}\left(\left(\left\langle V_{b}\right\rangle+\frac{Q-C_{p}\left(2\left\langle V_{b}\right\rangle+4 V_{d}\right)}{C_{b}}\right)^{2}-\left\langle V_{b}\right\rangle^{2}\right)
$$

As the voltage drop $V_{d}$ is small enough to be negligible,

$$
E_{\text {com }}=2\left(\frac{C_{p}}{C_{b}}-1\right) C_{p}\left\langle V_{b}\right\rangle^{2}+\left(1-\frac{2 C_{p}}{C_{b}}\right) Q\left\langle V_{b}\right\rangle+\frac{Q^{2}}{2 C_{b}}
$$

Given $C_{b} \gg C_{p}$,

$$
E_{c o m}=-2 C_{p}\left\langle V_{b}\right\rangle^{2}+Q\left\langle V_{b}\right\rangle+\frac{Q^{2}}{2 C_{b}}
$$

The total generated energy in each force cycle should be the total energy increment during compression and release. For the direct charge topology with full-wave rectifier, the energy stored during compression $E_{c o m}$ and the energy stored during release $E_{r e l}$ should be the same. Thus, the total stored energy should be:

$$
E=E_{c o m}+E_{r e l}=2 E_{c o m}=-4 C_{p}\left\langle V_{b}\right\rangle^{2}+2 Q\left\langle V_{b}\right\rangle+\frac{Q^{2}}{C_{b}}
$$

Equation (4.9) is quadratic and the maximum voltage of the bucket capacitor that can be reached is

$$
\left\langle V_{b}\right\rangle_{\max }=\frac{Q}{2 C_{p}}
$$

which is half of the open circuit voltage of the piezoelectric unit. To maximize the energy increment $E$, the optimal rectified voltage should be: 


$$
\left\langle V_{b}\right\rangle_{o p t}=\frac{-2 Q}{2 \times\left(-4 C_{p}\right)}=\frac{Q}{4 C_{p}}
$$

Thus bucket capacitor voltage should be rectified to one quarter of the open-circuit piezoelectric voltage peak to achieve the highest energy increment $E_{\max }$.

$$
E_{\text {max }}=\frac{Q^{2}}{4 C_{p}}+\frac{Q^{2}}{C_{b}} \approx \frac{Q^{2}}{4 C_{p}}
$$

Figure 4.4 shows the relationship between the energy increment in each force cycle and the rectified voltage $\left\langle V_{b}\right\rangle$. It shows that the increased energy is low when $\left\langle V_{b}\right\rangle$ is low and increases with $\left\langle V_{b}\right\rangle$ until that $\left\langle V_{b}\right\rangle$ is one quarter of the peak open-circuit piezoelectric voltage $\frac{Q}{4 C_{p}}$, after that the energy increment keeps decreasing to 0 when $\left\langle V_{b}\right\rangle$ is $\frac{Q}{2 C_{p}}$.

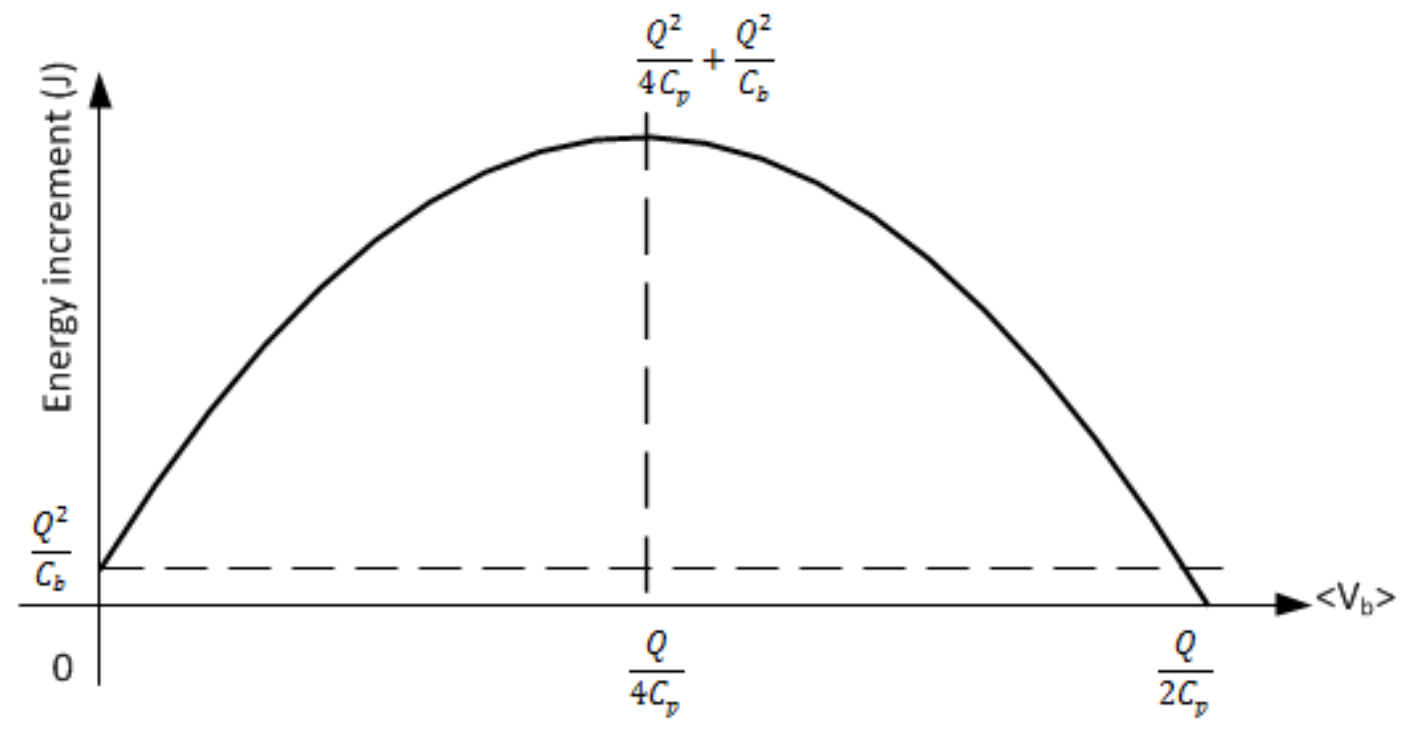

Figure 4.4 Energy increment in $C_{b}$ according to the rectified voltage $\left\langle V_{b}\right\rangle$. 


\subsubsection{Power output of the direct discharge through half-wave rectifier}

Direct discharge with half-wave rectifier (i.e., voltage doubler) is also a common topology in piezoelectric KEHSs [75]. Compared to the full-wave rectifier, the voltage doubler uses only two diodes, as shown in Figure 4.5.

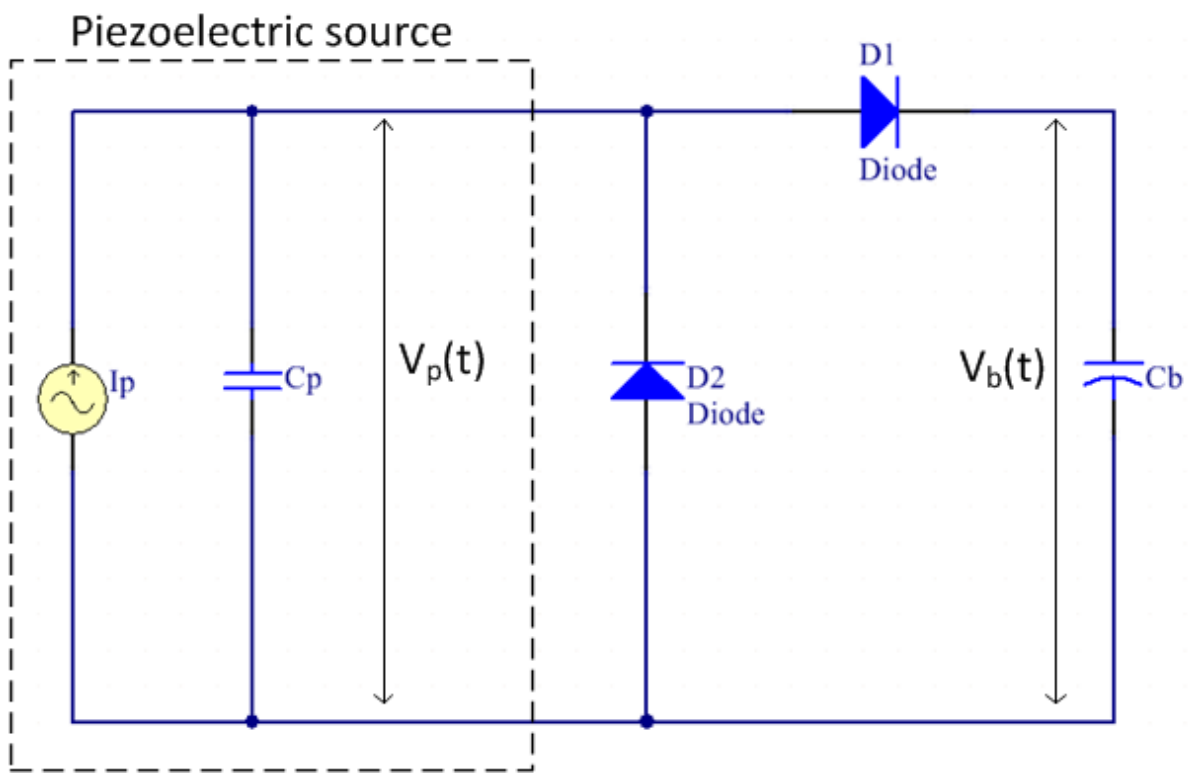

Figure 4.5 Equivalent circuit of the direct discharge topology with half-wave rectifier.

Figure 4.6 shows the corresponding waveforms of the piezoelectric current and voltage. During the positive current period, a part of the current is used to charge the piezoelectric internal capacitor $C_{p}$ from $0 \mathrm{~V}$ to $\left(\left\langle V_{b}\right\rangle+V_{d}\right)$, after which the current starts to flow to the bucket capacitor. During the inverted current period, the diode in parallel with the piezoelectric unit is forward biased, and the voltage across the piezoelectric unit $V_{p}(t)$ is discharged back to zero. Therefore there is no current flowing to the bucket capacitor during this period. 


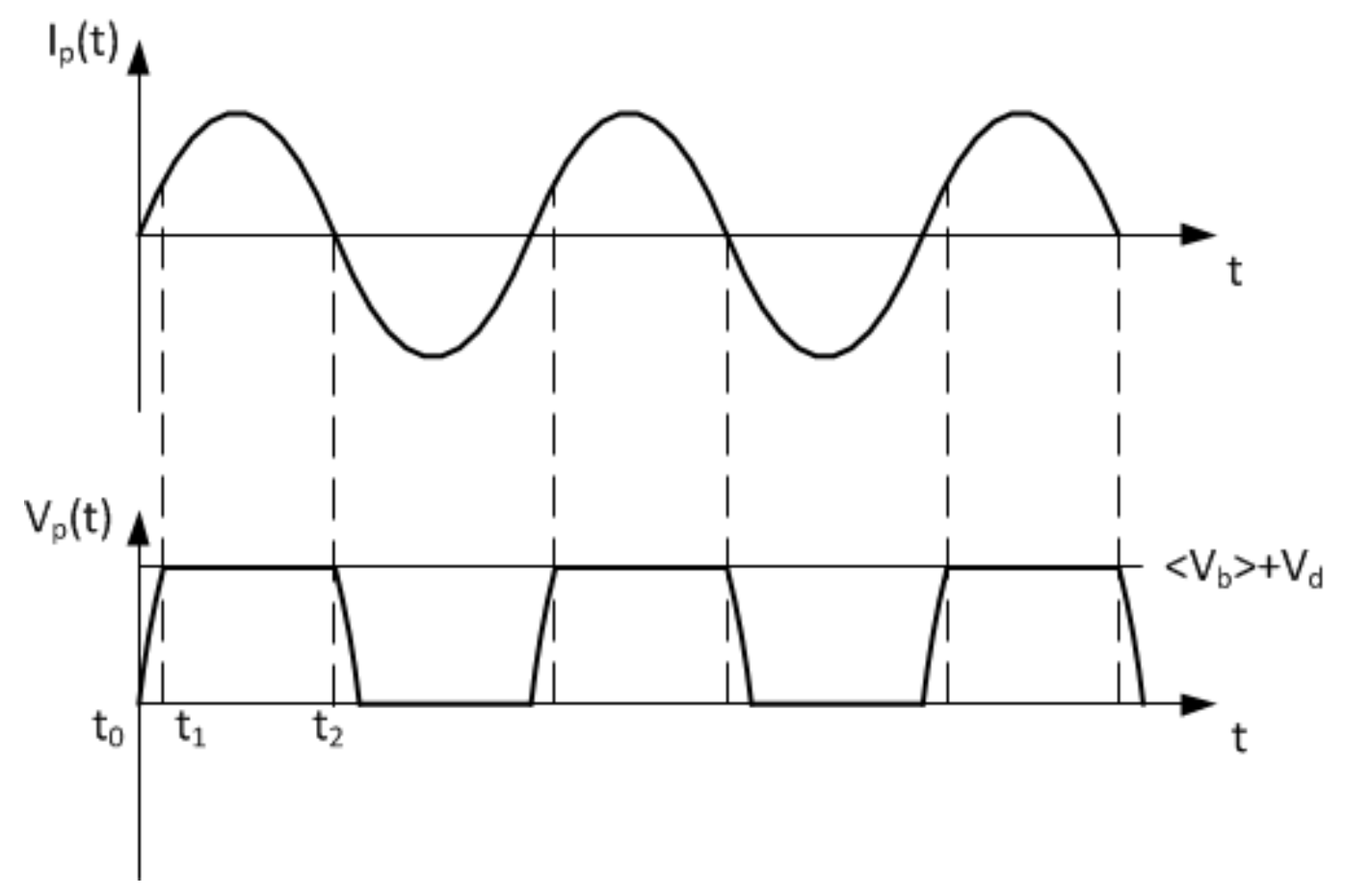

Figure 4.6 Waveforms of piezoelectric current and voltage of direct discharge topology with half-wave rectifier.

Given the voltage before and after each compression, the energy increment of the bucket capacitor can be calculated by Equation (4.4). $\left\langle V_{b}^{\prime}\right\rangle$ can be calculated by the bucket capacitor net charge increment, which is the total piezoelectric charge generated minus the charge wasted on charging the piezoelectric internal capacitor to the rectified voltage. Assuming $C_{b} \gg C_{p}$,

$$
\left\langle V_{b}\right\rangle^{\prime}=\left\langle V_{b}\right\rangle+\frac{Q-C_{p}\left(\left\langle V_{b}\right\rangle+V_{d}\right)}{C_{b}}
$$

Combining Equation (4.4) and (4.12),

$$
E_{\text {com }}=\frac{1}{2} C_{b}\left(\left(\left\langle V_{b}\right\rangle+\frac{Q-C_{p}\left(\left\langle V_{b}\right\rangle+V_{d}\right)}{C_{b}}\right)^{2}-\left\langle V_{b}\right\rangle^{2}\right)
$$

As the voltage drop $V_{d}$ is small enough to be negligible, 


$$
E_{c o m}=\left(\frac{C_{p}^{2}-2 C_{b} C_{p}}{2 C_{b}}\right)\left\langle V_{b}\right\rangle^{2}+\left(\frac{C_{b}-C_{p}}{C_{b}}\right) Q\left\langle V_{b}\right\rangle+\frac{Q^{2}}{2 C_{b}}
$$

Given $C_{b} \gg C_{p}$,

$$
E_{\text {com }}=-C_{p}\left\langle V_{b}\right\rangle^{2}+Q\left\langle V_{b}\right\rangle+\frac{Q^{2}}{2 C_{b}}
$$

It should be mentioned that the current flow from the half-wave rectifier does not occur every half force cycle as the full-wave rectifier does. During force release period, the current from the half-wave is kept at zero, so the energy increment during release is zero, and the total energy increment during the full force cycle is:

$$
\begin{aligned}
E & =E_{\text {com }}+E_{\text {rel }}=E_{\text {com }}+0 \\
& =-C_{p}\left\langle V_{b}\right\rangle^{2}+Q\left\langle V_{b}\right\rangle+\frac{Q^{2}}{2 C_{b}}
\end{aligned}
$$

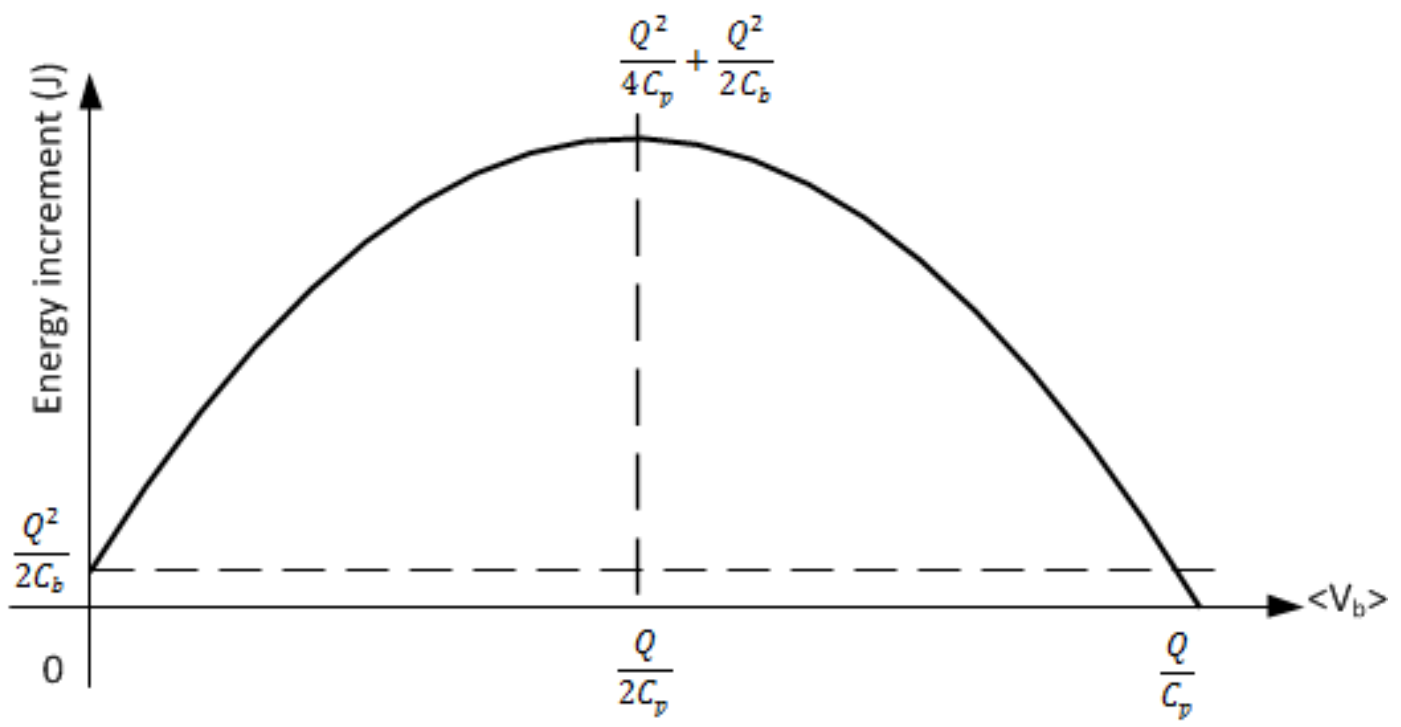

Figure 4.7 Energy increment in $C_{b}$ according to the rectified voltage $\left\langle V_{b}\right\rangle$. 
As shown in Figure 4.7, Equation (4.15) is quadratic, and the maximum voltage the bucket capacitor can be rectified to is:

$$
\left\langle V_{b}\right\rangle_{\max }=\frac{Q}{C_{p}}
$$

which is the open circuit voltage of the piezoelectric unit. To maximize the energy increment $E$, the optimal rectified voltage should be:

$$
\left\langle V_{b}\right\rangle_{o p t}=\frac{Q}{2 C_{p}}
$$

Thus bucket capacitor voltage should be rectified to half of the peak open-circuit voltage of the piezoelectric units, and the maximum energy increment in the bucket capacitor during a force cycle is:

$$
\begin{aligned}
E_{\text {max }} & =-C_{p}\left\langle V_{b}\right\rangle_{o p t}{ }^{2}+Q\left\langle V_{b}\right\rangle_{o p t}+\frac{Q^{2}}{2 C_{b}} \\
& =\frac{Q^{2}}{4 C_{p}}+\frac{Q^{2}}{2 C_{b}} \approx \frac{Q^{2}}{4 C_{p}}
\end{aligned}
$$

Compared to the full-wave rectifier, the half-wave rectifier has two less diodes. If the effects of diodes are ignored, the maximum rectified voltage of the half-wave rectifier circuit is double that of the full-wave rectifier circuit. Although the maximum power of the two topologies is the same, the half-wave rectifier circuit requires a rectified voltage double that of the full-wave rectifier in order to obtain the same maximum power. 


\subsection{Nonlinear switching topology}

The main disadvantage of the direct discharge topology is that a considerable part of the generated current is wasted in the process of charging and discharging $C_{P}$. Although the half-wave rectifier direct discharge topology lowers the charging and discharging voltage of $C_{p}$ during compression by half, energy is not collected when the load force decreases. Additionally, for the direct discharge topology, reaching the maximum harvesting power requires the bucket capacitor be maintained with at least one quarter of the open circuit voltage, which could be over one hundred volts and difficult to reach in terms of required charging time. For most low power consuming electronic devices like wireless sensors, the nominal voltage is only from $3 \mathrm{~V}$ to $5 \mathrm{~V}$. In addition, if the rectified voltage is far below the optimal rectified voltage, the power efficiency will be significantly affected.

As proven in the electromechanical model section, $V_{p}(t)$ and $I_{p}(t)$ should have as small phase shift as possible and $V_{p}(t)$ should be as large as possible. According to the corresponding waveforms (Figure 4.3 and Figure 4.6) of direct discharge topologies, the phase shift $\theta$ between $V_{p}(t)$ and $I_{p}(t)$ is determined by the process of charging and discharging the piezoelectric capacitor $C_{p}$. An increase of the rectified voltage $\left\langle V_{b}\right\rangle$ not only leads to an increase of $V_{p}(t)$, which increases the power efficiency, but also leads to an increase of the phase shift $\theta$ (i.e., more time to discharge $C_{p}$ ), which decreases the power efficiency. 
To maximize the output piezoelectric power, the nonlinear switching topology is therefore designed to shape the voltage across the piezoelectric unit $V_{p}(t)$ in a way that reduces the phase shift $\theta$ and increases $V_{p}(t)$ simultaneously. Five recent nonlinear switching topologies are studied below.

\subsubsection{Synchronized switch harvesting on inductor (SSHI) topology}

The SSHI topology uses an inductor and a switch connected in series to form an oscillator with the piezoelectric internal capacitor $C_{p}$. The oscillator is able to invert the voltage of $C_{p}$ every time the switch is turned on for a certain period of time. Considering the energy equation from the electromechanical model, the voltage inversion decreases the phase shift $\theta$ of $I_{p}(t)$ and increases $V(t)$. There are two types of SSHI topologies: Series SSHI and parallel SSHI.

\subsubsection{Series SSHI topology}

The series SSHI topology is shown in Figure 4.8, where the inductor and the switch connect with the piezoelectric generator in series [76, 77]. 


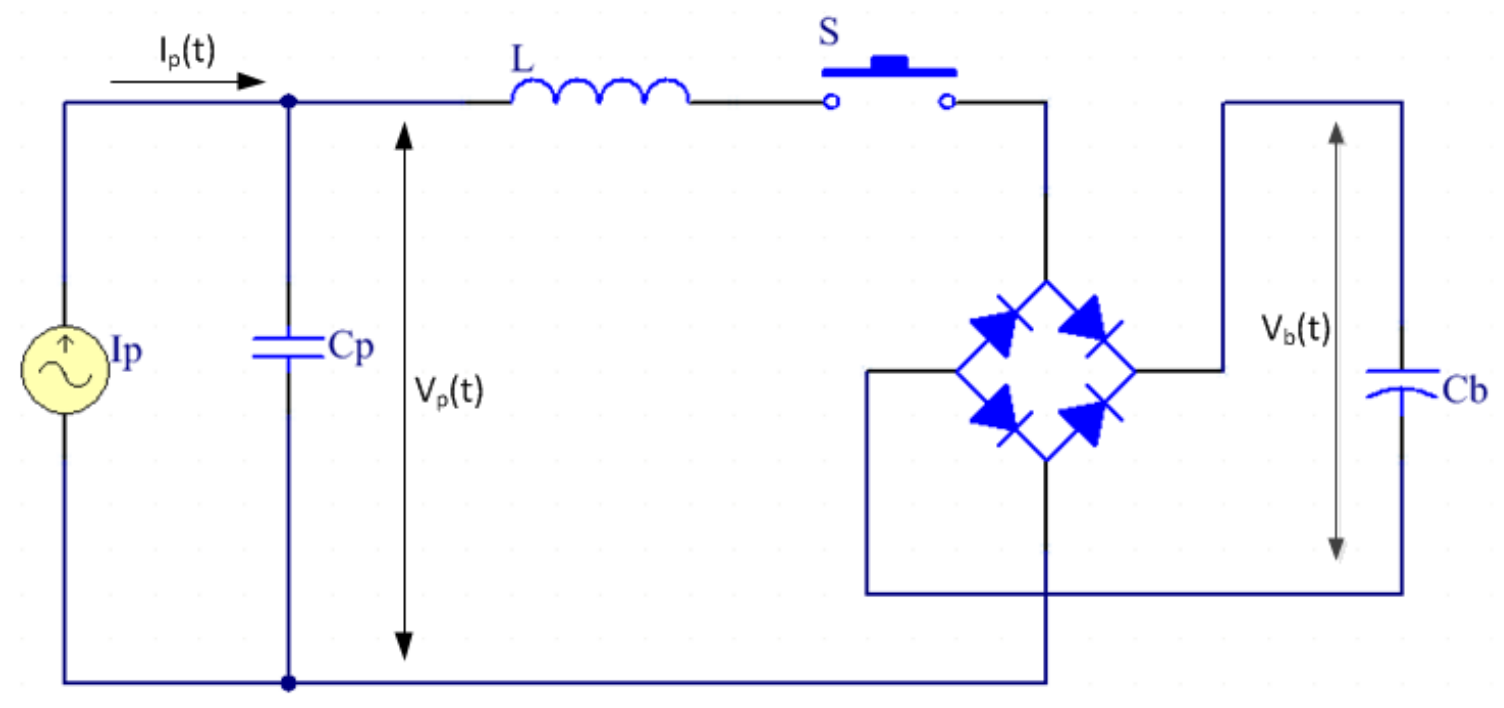

Figure 4.8 Electric circuit of the series SSHI topology.

The switch is kept open and the piezoelectric generator is in an open circuit condition until the piezoelectric current $I_{p}(t)$ changes direction, at which time the piezoelectric open circuit voltage $V_{p}(t)$ increases from $\left\langle V_{p}^{\prime}\right\rangle$ to $\left\langle V_{p}\right\rangle$. As the switch turns on, the piezoelectric energy starts to transfer from the piezoelectric capacitor $C_{P}$ to the bucket capacitor $C_{b}$, and the voltage inversion of $C_{p}$ occurs. It should be noted that this is a simplified circuit model. In reality there should be a voltage transformer before the fullwave rectifier [78], so $V_{p}$ can be inverted below zero without being interrupted by the rectifier. The waveforms of the piezoelectric current and voltage are shown in Figure 4.9. 


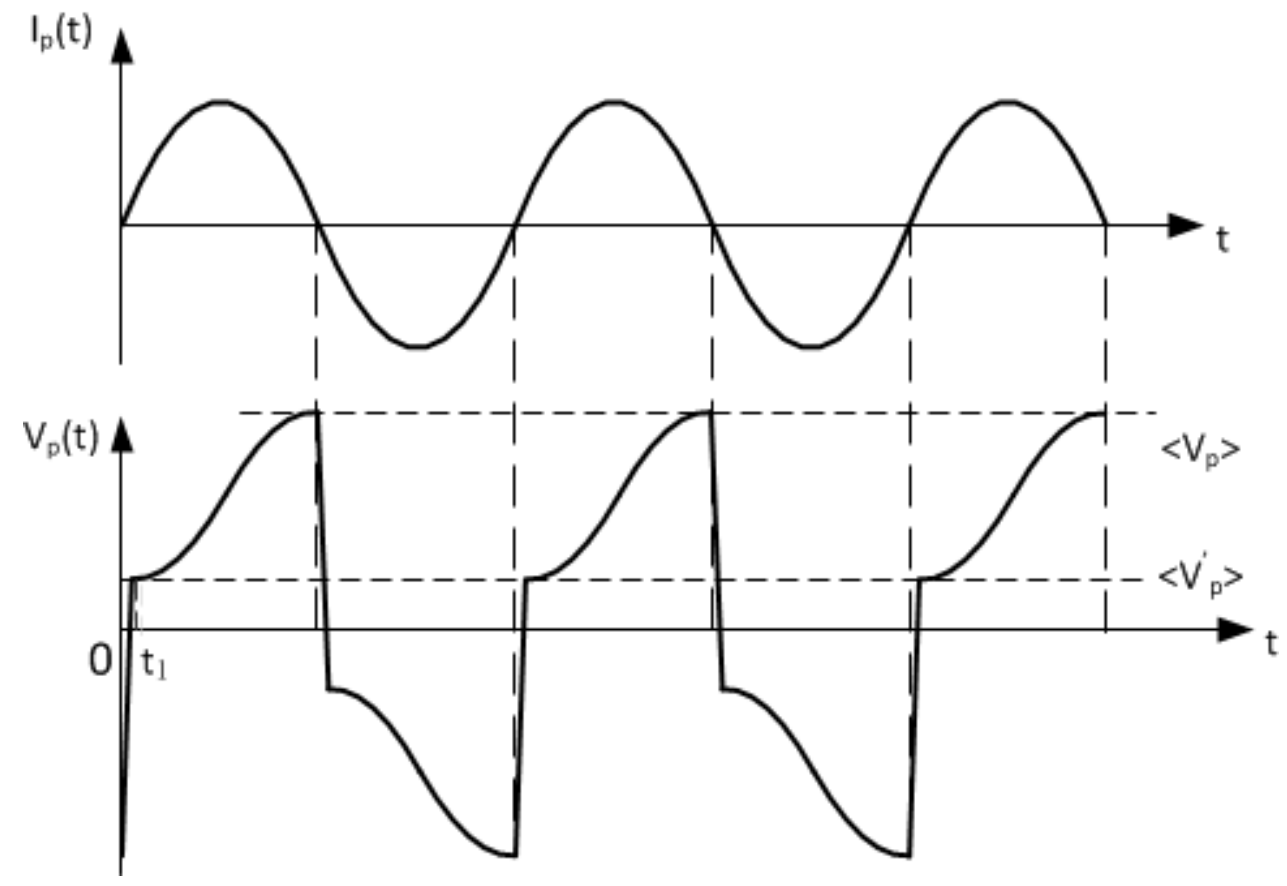

Figure 4.9 Waveforms of piezoelectric current and voltage of the Series SSHI.

When the switch closes, a second order RLC circuit is formed by the piezoelectric capacitor $C_{p}$, the inductor $L$ and its dissipation resistor $R$, and the bucket capacitor $C_{b}$, as illustrated in Figure 4.10.

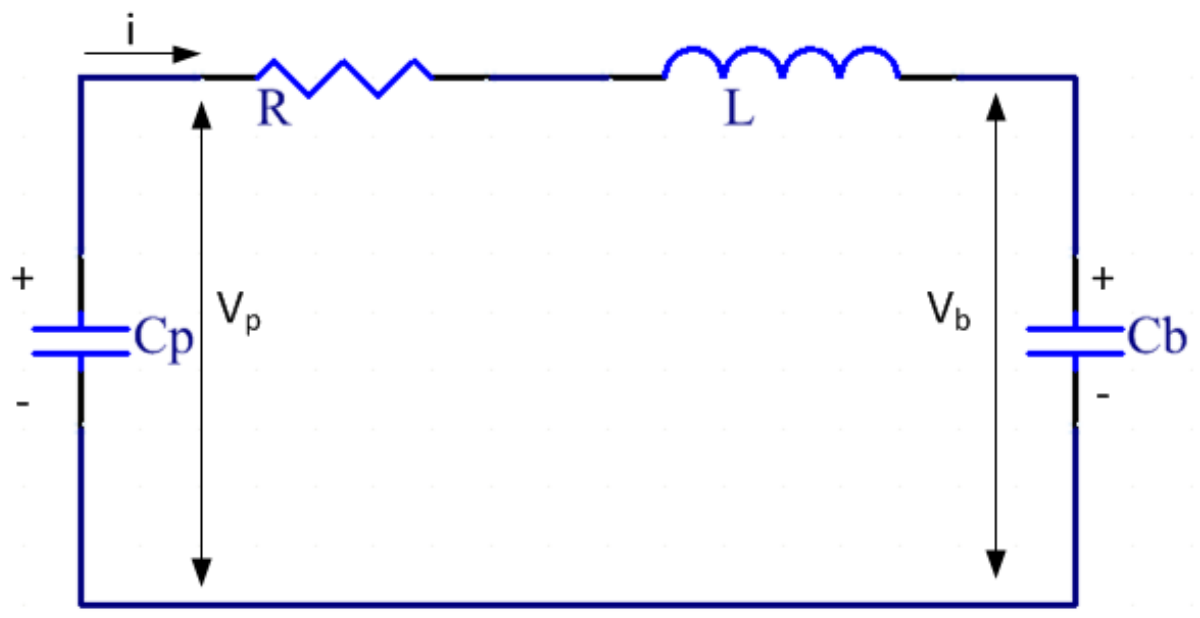

Figure 4.10 Second order RLC circuit. 
Given that the piezoelectric open circuit voltage peaks at $\left\langle V_{p}\right\rangle$ before the switch's closure, and the voltage of $C_{b}$ is $\left\langle V_{b}\right\rangle$, assuming the generated current is continuous and the charging process is stable (the absolute value of the inverted piezoelectric voltage during the compression and the release are the same), the inverted voltage of $C_{p}$ can be calculated as follows:

Using Kirchoff's voltage law across the RLC circuit loop,

$$
V_{p}(t)=R i(t)+L \frac{d i(t)}{d t}+V_{b}(t)
$$

Taking the derivative of both sides with respect to $t$,

$$
\frac{d V_{p}(t)}{d t}=R \frac{d i(t)}{d t}+L \frac{d^{2} i(t)}{d t^{2}}+\frac{d V_{b}(t)}{d t}
$$

The current through $C_{P}$ and $C_{b}$ is $i(t)$,

$$
i(t)=-C_{P} \frac{d V_{p}(t)}{d t}=C_{b} \frac{d V_{b}(t)}{d t}
$$

Combining Equation (4.20) and (4.21), we obtain

$$
L C_{s} \frac{d^{2} i(t)}{d t^{2}}+R C_{s} \frac{d i(t)}{d t}+i(t)=0
$$

where $C_{s}=\frac{C_{p} C_{b}}{C_{p}+C_{b}}$ is the capacitance of $C_{p}$ and $C_{b}$ in series. When $C_{b} \gg C_{p}, C_{s} \approx C_{p}$. Equation (4.22) is a second order homogeneous equation with constant coefficients, and has the general solution when $\frac{L}{C_{p}}>\frac{R^{2}}{4}\left(\right.$ i.e. $\left.\frac{\mathrm{R}^{2} C_{p}}{4 L}<1\right)$.

$$
i(t)=c_{1} e^{-\alpha t} \cos (\omega t)+c_{2} e^{-\alpha t} \sin (\omega t)
$$


where $\alpha=\frac{R}{2 L}, \omega=\sqrt{\frac{1}{L C_{p}}-\frac{R^{2}}{4 L^{2}}}$. Assuming $\frac{\mathrm{R}^{2} C_{p}}{4 L} \approx 0$, then we have $\omega=\sqrt{\frac{1}{L C_{p}}}$. Given the initial conditions $i(0)=0, V_{p}(0)=\left\langle V_{p}\right\rangle, V_{b}(0)=\left\langle V_{b}\right\rangle$, and Equation (4.23), the coefficients $c_{1}$ and $c_{2}$ can be calculated.

$$
c_{1}=0, c_{2}=\frac{\left\langle V_{p}\right\rangle-\left\langle V_{b}\right\rangle}{L \omega}
$$

so

$$
i(t)=\frac{\left\langle V_{p}\right\rangle-\left\langle V_{b}\right\rangle}{L \omega} e^{-\alpha t} \sin (\omega t)
$$

From Equation (4.21) and (4.25), we obtain

$$
V_{b}=-\frac{C_{p}}{C_{b}} V_{p}(t)+\frac{C_{p}}{C_{b}}\left\langle V_{p}\right\rangle+\left\langle V_{b}\right\rangle
$$

Inserting (4.25) and (4.26) to Equation (4.19) yields,

$$
\begin{aligned}
& V_{p}(t)=\frac{C_{p}}{C_{b}+C_{p}}\left\langle V_{p}\right\rangle+\frac{C_{b}}{C_{p}+C_{b}}\left\langle V_{b}\right\rangle \\
& \quad+\frac{C_{b}}{C_{b}+C_{p}}\left(\left\langle V_{p}\right\rangle-\left\langle V_{b}\right\rangle\right) e^{-\alpha t}\left[\cos (\omega t)+\frac{\mathrm{R}}{2 \omega L} \sin (\omega t)\right] \\
& =\frac{C_{p}}{C_{b}+C_{p}}\left\langle V_{p}\right\rangle+\frac{C_{b}}{C_{p}+C_{b}}\left\langle V_{b}\right\rangle+\frac{C_{b}}{C_{b}+C_{p}}\left(\left\langle V_{p}\right\rangle-\left\langle V_{b}\right\rangle\right) \beta e^{-\alpha t} \cos (\omega t-\phi)
\end{aligned}
$$

where $\beta=\sqrt{\frac{R^{2}}{4 \omega^{2} L^{2}}+1}, \phi=\tan ^{-1}\left(\frac{\mathrm{R}}{2 \omega L}\right)=\tan ^{-1}\left(\sqrt{\frac{R^{2} C_{p}}{4 L}}\right)$.

Combining Equation (4.27) with Equation (4.26) yields 


$$
\begin{gathered}
V_{b}(t)=\frac{C_{p}}{C_{b}+C_{p}}\left\langle V_{p}\right\rangle+\frac{C_{b}}{C_{p}+C_{b}}\left\langle V_{b}\right\rangle \\
\quad-\frac{C_{p}}{C_{b}+C_{p}}\left(\left\langle V_{p}\right\rangle-\left\langle V_{b}\right\rangle\right) e^{-\alpha t}\left[\cos (\omega t)+\frac{\mathrm{R}}{2 \omega L} \sin (\omega t)\right] \\
=\frac{C_{p}}{C_{b}+C_{p}}\left\langle V_{p}\right\rangle+\frac{C_{b}}{C_{p}+C_{b}}\left\langle V_{b}\right\rangle-\frac{C_{p}}{C_{b}+C_{p}}\left(\left\langle V_{p}\right\rangle-\left\langle V_{b}\right\rangle\right) \beta e^{-\alpha t} \cos (\omega t-\phi)
\end{gathered}
$$

From Equation (4.21) and (4.25), we further obtain

$$
\left\{\begin{array}{c}
\frac{d V_{b}(t)}{d t}=\frac{i(t)}{C_{b}}=\frac{\left\langle V_{p}\right\rangle-\left\langle V_{b}\right\rangle}{L \omega C_{b}} e^{-\alpha t} \sin (\omega t) \\
\frac{d V_{p}(t)}{d t}=-\frac{i(t)}{C_{p}}=-\frac{\left\langle V_{p}\right\rangle-\left\langle V_{b}\right\rangle}{L \omega C_{p}} e^{-\alpha t} \sin (\omega t)
\end{array}\right.
$$

The second peaks of $V_{b}(t)$ and $V_{p}(t)$ occur at $\frac{\pi}{\omega}$ :

$$
\left\{\begin{array}{l}
V_{p}\left(\frac{\pi}{\omega}\right)=\frac{C_{p}}{C_{b}+C_{p}}\left\langle V_{p}\right\rangle+\frac{C_{b}}{C_{p}+C_{b}}\left\langle V_{b}\right\rangle-\frac{C_{b}}{C_{b}+C_{p}}\left(\left\langle V_{p}\right\rangle-\left\langle V_{b}\right\rangle\right) \gamma \\
V_{b}\left(\frac{\pi}{\omega}\right)=\frac{C_{p}}{C_{b}+C_{p}}\left\langle V_{p}\right\rangle+\frac{C_{b}}{C_{p}+C_{b}}\left\langle V_{b}\right\rangle+\frac{C_{p}}{C_{b}+C_{p}}\left(\left\langle V_{p}\right\rangle-\left\langle V_{b}\right\rangle\right) \gamma
\end{array}\right.
$$

where $\gamma=e^{-\frac{\alpha \pi}{\omega}}$ and $\gamma \in(0,1)$. When $C_{b} \gg C_{p}$, then

$$
\left\langle V_{p}^{\prime}\right\rangle=V_{p}\left(\frac{\pi}{\omega}\right)=(1+\gamma)\left\langle V_{b}\right\rangle-\gamma\left\langle V_{p}\right\rangle
$$

Because $\left\langle V_{p}\right\rangle=-\left\langle V_{p}^{\prime}\right\rangle+\frac{Q}{C_{p}}$, Equation (4.31) becomes:

$$
\left\langle V_{p}\right\rangle=\frac{\gamma+1}{\gamma-1}\left\langle V_{b}\right\rangle+\frac{Q}{C_{p}(1-\gamma)}
$$

Assuming $\mathrm{C}_{\mathrm{b}} \gg C_{p}$, the energy increment in the bucket capacitor during the compression should be:

$$
E_{\text {com }}=\frac{C_{b}}{2}\left[\left(V_{b}\left(\frac{\pi}{\omega}\right)\right)^{2}-\left\langle V_{b}\right\rangle^{2}\right]
$$




$$
\begin{gathered}
=\frac{C_{b}}{2}\left[\left(\frac{\left(C_{b}-C_{p} \gamma\right)^{2}}{\left(C_{b}+C_{p}\right)^{2}}-1\right)\left\langle V_{b}\right\rangle^{2}+\frac{2(1+\gamma) C_{p}\left(C_{b}-C_{p} \gamma\right)}{\left(C_{b}+C_{p}\right)^{2}}\left\langle V_{p}\right\rangle\left\langle V_{b}\right\rangle+\frac{(1+\gamma)^{2} C_{p}^{2}}{\left(C_{b}+C_{p}\right)^{2}}\left\langle V_{p}\right\rangle^{2}\right] \\
=\frac{-2(1+\gamma) C_{p}}{(1-\gamma)}\left\langle V_{b}\right\rangle^{2}+\frac{(1+\gamma) Q}{(1-\gamma)}\left\langle V_{b}\right\rangle+\frac{(1+\gamma)^{2} Q^{2}}{2(1-\gamma)^{2} C_{b}}
\end{gathered}
$$

The energy increment in the full force cycle is given by:

$$
\begin{aligned}
E & =E_{\text {com }}+E_{\text {rel }}=2 E_{\text {com }} \\
& =\frac{-4(1+\gamma) C_{p}}{(1-\gamma)}\left\langle V_{b}\right\rangle^{2}+\frac{2(1+\gamma) Q}{(1-\gamma)}\left\langle V_{b}\right\rangle+\frac{(1+\gamma)^{2} Q^{2}}{(1-\gamma)^{2} C_{b}}
\end{aligned}
$$

As shown in Figure 4.11, Equation (4.34) is quadratic, and the maximum voltage the bucket capacitor can be rectified to is:

$$
\left\langle V_{b}\right\rangle_{\max }=\frac{Q}{2 C_{p}}
$$

which is half the open circuit voltage of the piezoelectric unit. To maximize the stored energy $E$, the optimal rectified voltage should be:

$$
\left\langle V_{b}\right\rangle_{o p t}=\frac{Q}{4 C_{p}}
$$

Therefore the bucket capacitor voltage should be rectified to one quarter of the peak open-circuit voltage of the piezoelectric units, and in this case, the maximum energy increment of the bucket capacitor in the force cycle is:

$$
E_{\text {max }}=\frac{(1+\gamma) Q^{2}}{4(1-\gamma) C_{p}}\left\langle V_{b}\right\rangle+\frac{(1+\gamma)^{2} Q^{2}}{(1-\gamma)^{2} C_{b}}
$$

It should be mentioned that as $\gamma$ approaches 1 , the piezoelectric voltage $\left\langle V_{p}\right\rangle$ just before switching will approach infinity in this ideal model, according to Equation (4.32). In 
actuality, the piezoelectric voltage has a limit (i.e., its poling voltage), over which the piezoelectric material will be depolarized, so the energy increment will not be unlimited. Compared with the direct charge topology, the maximum power of the series SSHI is expected to be higher depending on $\gamma$.

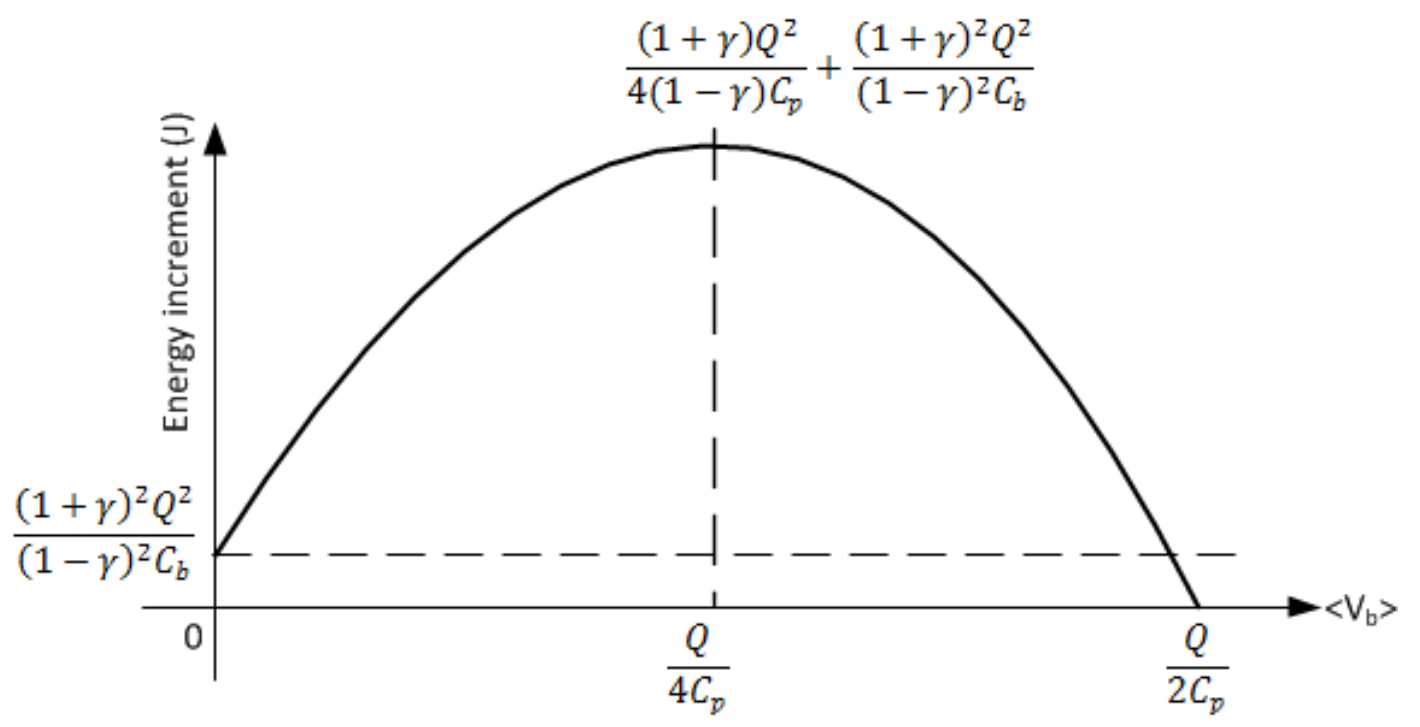

Figure 4.11 Energy increment in $C_{b}$ according to the rectified voltage $\left\langle V_{b}>\right.$.

\subsubsection{Parallel SSHI topology}

The parallel SSHI is an improvement of the switch-only rectifier and uses an additional inductor in series with the switch [59, 79]. Its circuit model, shown in Figure 4.12, is similar to the series SSHI, except that the inductor and switch are connected in parallel with the piezoelectric source. It is able to invert the piezoelectric voltage when the piezoelectric current changes direction, and therefore the piezoelectric charges wasted on charging and discharging the piezoelectric capacitor $C_{p}$ is saved. Considering the energy 
equation from the electromechanical model, the phase difference $\theta$ between $I_{p}$ and $V_{p}$ is reduced considering that the voltage inversion process is faster.

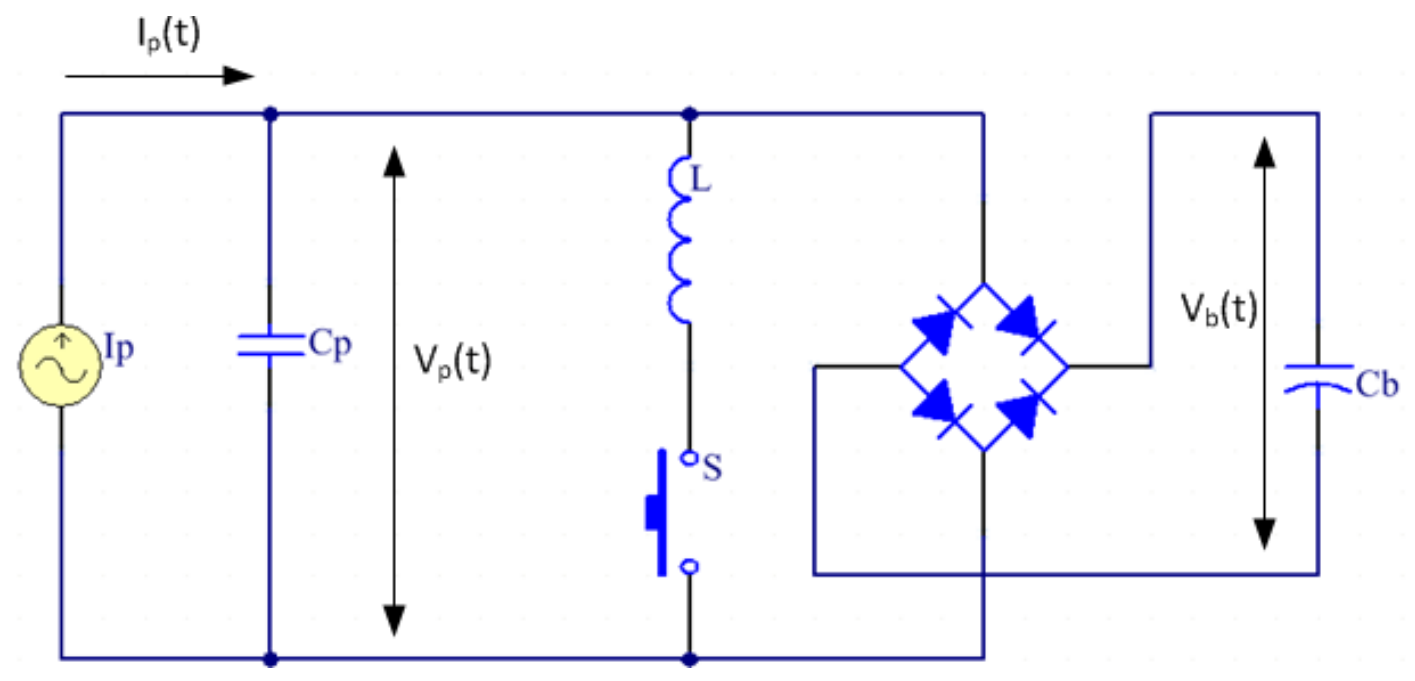

Figure 4.12 Electric circuit of the parallel SSHI.

As shown in Figure 4.13, whenever the piezoelectric current $I_{p}$ changes sign, the switch is turned on for a period of time from $t_{1}$, in which the voltage of $C_{p}$ is inverted through the oscillator formed by $C_{p}$ and $L$. The time interval $t_{1}$ for the voltage reversing is equal to half of the oscillator period,

$$
t_{1}=\frac{2 \pi \sqrt{L C_{p}}}{2}=\pi \sqrt{L C_{p}}
$$

$t_{1}$ should be as small as possible in order to decrease the phase shift $\theta$, thus the voltage reversing time interval $t_{1}$ should be adjusted to a level that is much smaller than the period of the cyclic load. As $C_{p}$ is known, the adjustment can be done by properly selecting $L$. 


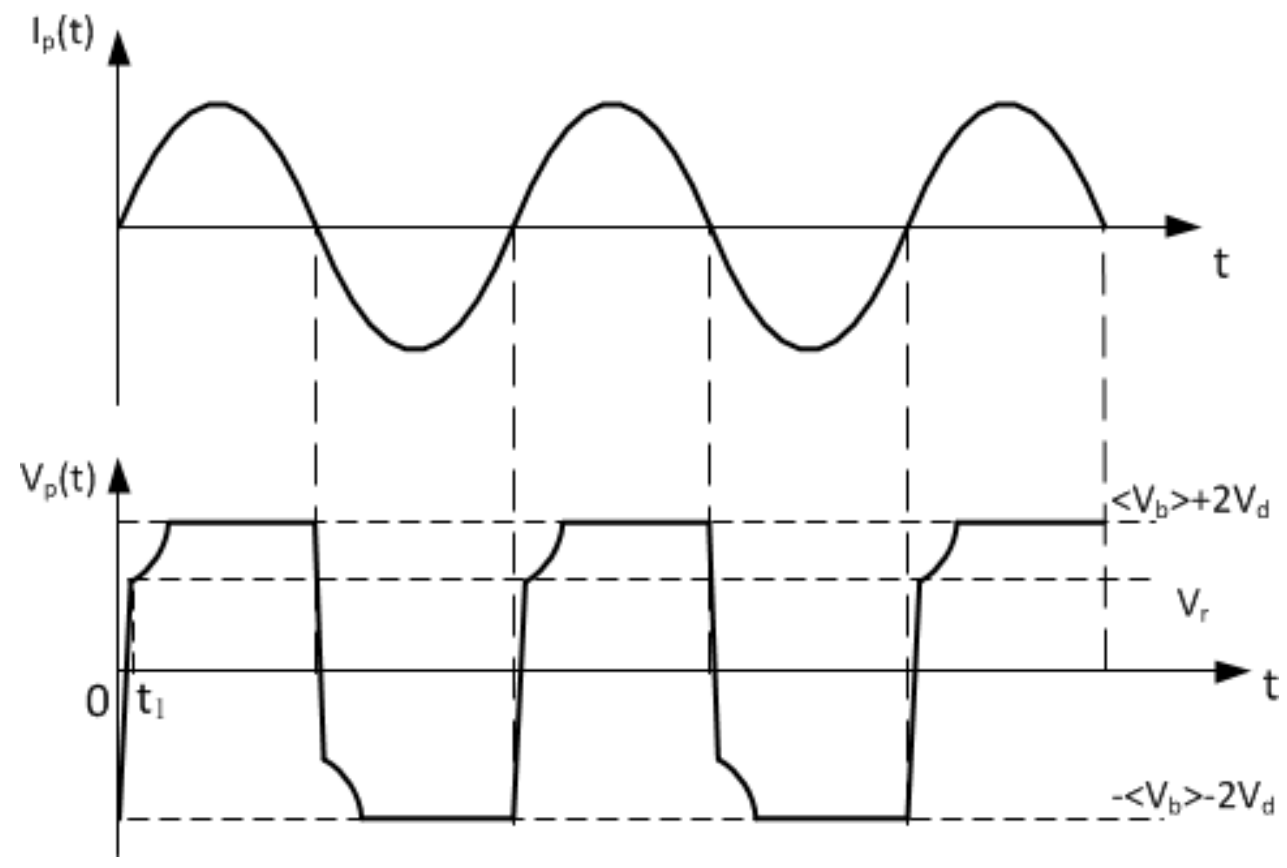

Figure 4.13 Waveforms of piezoelectric current and voltage of the Parallel SSHI.

The inverted voltage will not be exactly the opposite of the voltage before inverting because the inductor $L$ is not ideal and has an internal resistance $R$, which forms a second order RLC circuit as shown in Figure 4.14. This RLC circuit is similar to the RLC circuit given in Figure 4.10, and $V_{p}(t)$ can be calculated if $C_{b}=\infty$ and $\left\langle V_{b}\right\rangle=0$. Assuming the inductor of both circuits are the same, from Equation (4.30),

$$
V_{p}\left(\frac{\pi}{\omega}\right)=-\left\langle V_{b}\right\rangle \gamma
$$

where $\gamma=e^{-\frac{\alpha \pi}{\omega}}, \alpha=\frac{R}{2 L}$, and,$\omega=\sqrt{\frac{1}{L C_{p}}}$. 


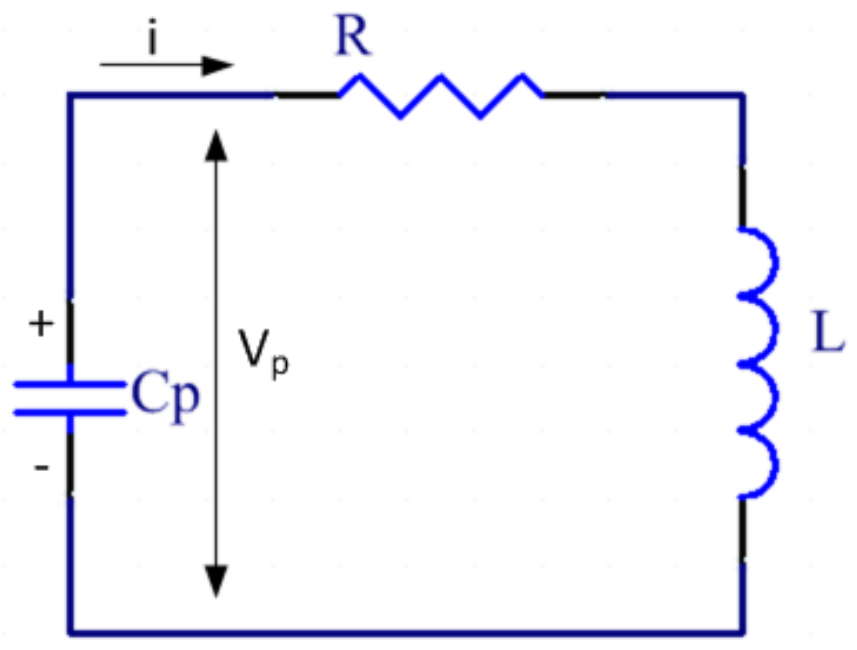

Figure 4.14 Second order RLC circuit.

Given the voltage before and after each compression, the energy increment of the bucket capacitor can be calculated by Equation (4.4). Assuming $C_{b} \gg C_{p}$ and $t_{1}$ is negligible, the increased voltage of $C_{b}$ is:

$$
\left\langle V_{b}^{\prime}\right\rangle=\left\langle V_{b}\right\rangle+\frac{Q-(1-\gamma)\left\langle V_{b}\right\rangle C_{p}}{C_{b}}
$$

Combining Equation (4.4) and (4.39), the energy increment during compression is:

$$
E_{\text {com }}=\frac{1}{2} C_{b}\left(\left(\left\langle V_{b}\right\rangle+\frac{Q-(1-\gamma)\left\langle V_{b}\right\rangle C_{p}}{C_{b}}\right)^{2}-\left\langle V_{b}\right\rangle^{2}\right)
$$

Assuming $C_{b} \gg C_{p}$,

$$
E_{\text {com }}=-(1-\gamma) C_{p}\left\langle V_{b}\right\rangle^{2}+Q\left\langle V_{b}\right\rangle+\frac{Q^{2}}{2 C_{b}}
$$

The energy increment during the full force cycle is:

$$
E=E_{\text {com }}+E_{\text {rel }}=2 E_{\text {com }}
$$




$$
=-2(1-\gamma) C_{p}\left\langle V_{b}\right\rangle^{2}+2 Q\left\langle V_{b}\right\rangle+\frac{Q^{2}}{C_{b}}
$$

As shown in Figure 4.15, Equation (4.42) is quadratic, and the maximum voltage the bucket capacitor can be rectified to is related to $\gamma$ :

$$
\left\langle V_{b}\right\rangle_{\max }=\frac{Q}{(1-\gamma) C_{p}}
$$

which is more than the open circuit piezoelectric voltage, given $0<\gamma<1$. To maximize the stored energy $E$, the rectified voltage should be:

$$
\left\langle V_{b}\right\rangle_{o p t}=\frac{Q}{2(1-\gamma) C_{p}}
$$

Accordingly, the bucket capacitor voltage should be rectified to $\left\langle V_{b}\right\rangle_{o p t}$, and the maximum energy increment in the bucket capacitor during the force cycle is:

$$
E_{\max }=\frac{Q^{2}}{2(1-\gamma) C_{p}}+\frac{Q^{2}}{C_{b}}
$$

It should be mentioned that as $\gamma$ approaches 1 , the maximum voltage the bucket capacitor can be rectified to will become higher and higher. As mentioned in the series SSHI section, the piezoelectric voltage has a limit, and therefore the increased energy will not be unlimited. By comparing Equation (4.45) with Equation (4.36) from the series SSHI topology, we know that the maximum power of the parallel SSHI topology is higher than the series SSHI topology in theory. 


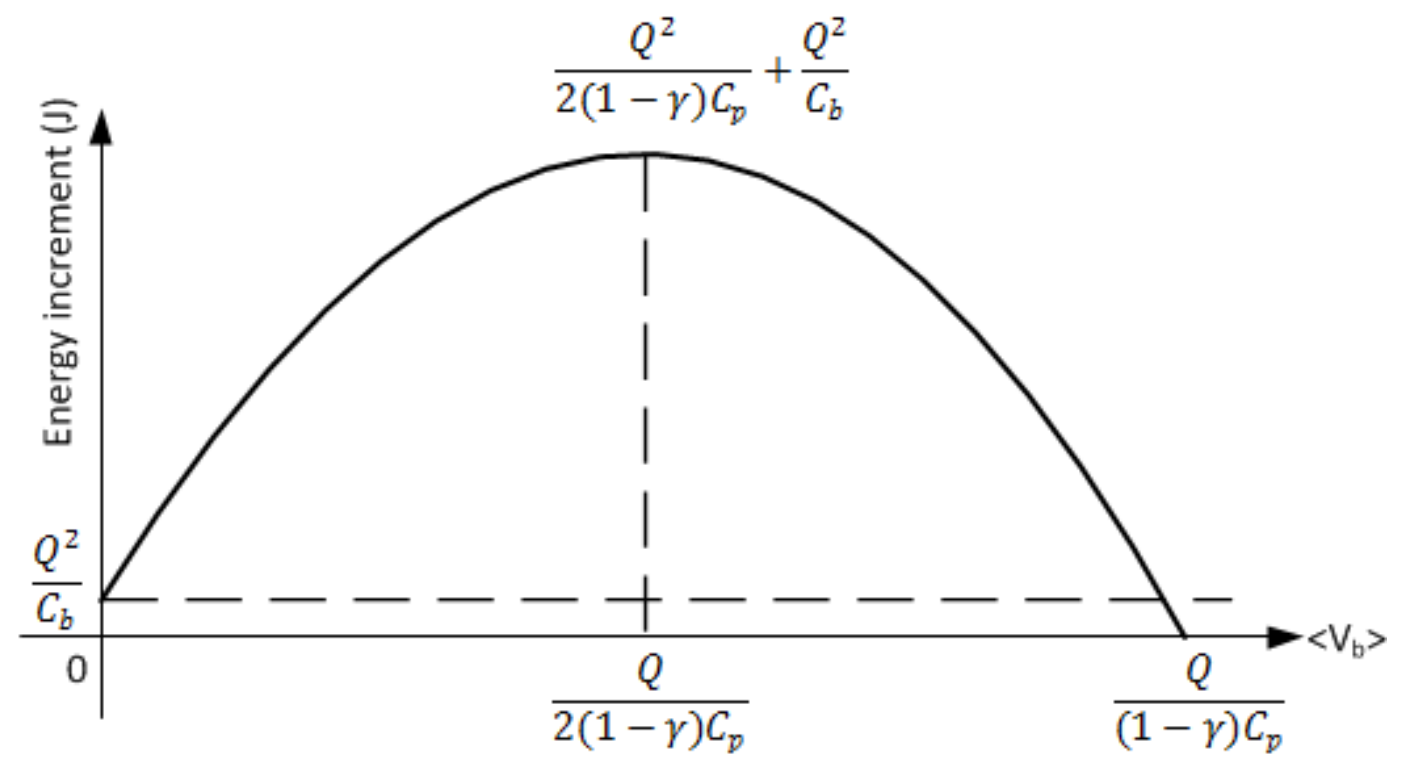

Figure 4.15 Energy increment in $C_{b}$ according to the rectified voltage $\left\langle V_{b}\right\rangle$.

\subsubsection{Synchronized Switching and Discharging to a storage Capacitor through an Inductor (SSDCI) topology}

The SSDCI topology has a similar architecture to the series SSHI [80], and its equivalent circuit of SSDCI topology is shown in Figure 4.16, where the switch and the inductor are connected after the rectification circuit. The working process of the SSDCI topology is similar to that of the series SSHI topology, as the switch is kept open until the piezoelectric current changes direction, and the piezoelectric voltage peaks. When the switch is closed, the piezoelectric energy starts to transfer from the piezoelectric capacitor to the bucket capacitor. However, the piezoelectric current is blocked by the full-wave rectifier when $V_{p}(t)$ is below the rectified voltage $\left\langle V_{b}\right\rangle$ of the bucket capacitor. Therefore, the voltage inversion stops when $V_{p}(t)$ equals $\left\langle V_{b}\right\rangle$, and then the switch opens. The energy left in the inductor then transfers to $C_{b}$ through a freewheeling diode. 


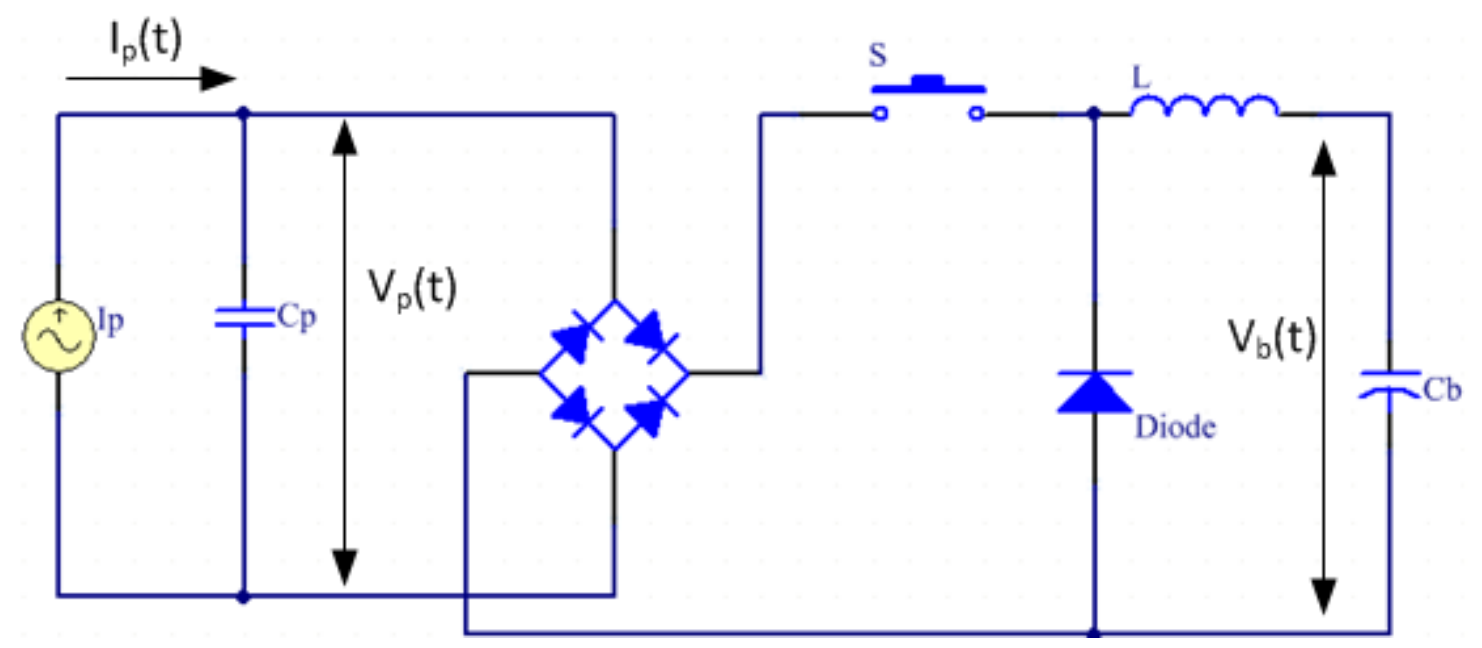

Figure 4.16 Electric circuit of the SSDCI topology.

The waveforms of the piezoelectric current and voltage is shown in Figure 4.17. Considering the energy Equation (4.3), the SSDCI topology is able to decrease the phase shift $\theta$ and increase the piezoelectric voltage $V_{p}$ at the same time. The phase shift $\theta$ is decreased because the charging process through the inductor is much faster, and the piezoelectric voltage $V_{p}$ is increased because the piezoelectric voltage peaks in the open circuit condition before the switch is closed in each cycle. 


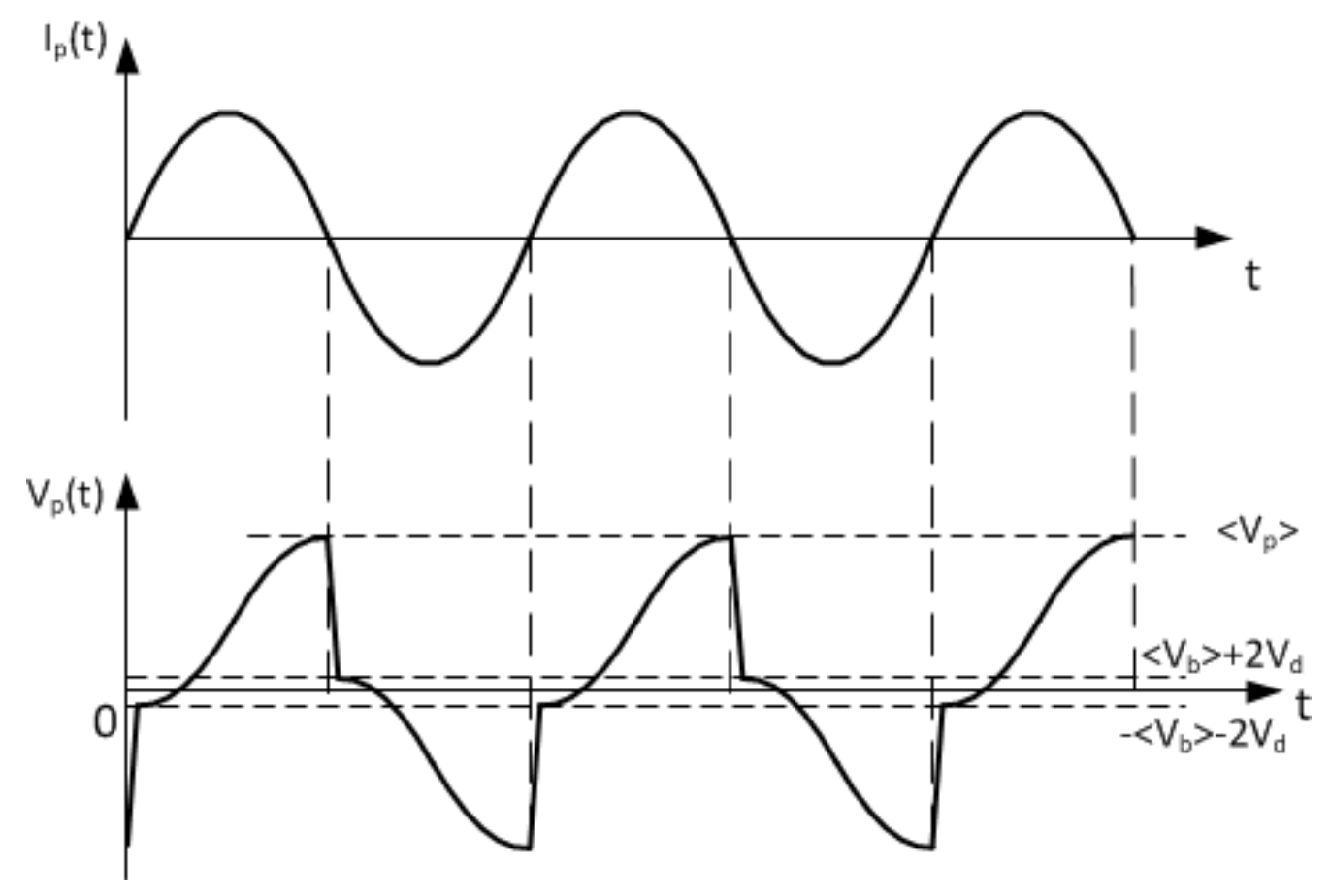

Figure 4.17 Waveforms of the SSDCI topology.

Similar to the series SSHI, when the switch is turned on, a second order RLC circuit is established as drawn in Figure 4.10. Given that the piezoelectric open circuit voltage peaks at $\left\langle V_{p}\right\rangle$ before the switch's closing, and the voltage of $C_{b}$ is $\left\langle V_{b}\right\rangle$, and assuming the switching process takes time $u$, when the switching process is interrupted, the voltage of the piezoelectric capacitor and the bucket capacitor should be the same. According to Equation (4.27) and (4.28), the increased voltage of the bucket capacitor $\left\langle V_{b}^{\prime}\right\rangle$ can be calculated as:

$$
V_{p}(u)=V_{b}(u)=\left\langle V_{b}^{\prime}\right\rangle=\frac{C_{p}}{C_{b}+C_{p}}\left\langle V_{p}\right\rangle+\frac{C_{b}}{C_{p}+C_{b}}\left\langle V_{b}\right\rangle
$$

Assuming $\frac{\mathrm{R}^{2} C_{p}}{4 L} \approx 0$, from Equation (4.27) and (4.28) we know 


$$
\phi=\tan ^{-1}\left(\sqrt{\frac{R^{2} C_{p}}{4 L}}\right) \approx 0
$$

and

$$
u=\frac{\pi+2 \phi}{2 \omega} \approx \frac{\pi}{2 \omega}
$$

According to Figure 4.17, it should be noted that the piezoelectric peak should be larger than the rectified voltage.

$$
\left\langle V_{p}\right\rangle=\left(\frac{Q}{C_{p}}-\left\langle V_{b}\right\rangle\right) \geq\left\langle V_{b}\right\rangle
$$

The range of the rectified voltage is:

$$
0 \leq\left\langle V_{b}\right\rangle \leq \frac{Q}{2 C_{p}}
$$

Assuming $C_{b} \gg C_{p}$, the energy increment of the bucket capacitor during the first time interval is:

$$
\begin{gathered}
E_{1}=\frac{1}{2} C_{b}\left(\left\langle V_{b}^{\prime}\right\rangle^{2}-\left\langle V_{b}\right\rangle^{2}\right) \\
=\frac{1}{2} C_{b}\left[\frac{-4 C_{b} C_{p}}{\left(C_{b}+C_{p}\right)^{2}}\left\langle V_{b}\right\rangle^{2}+\frac{2\left(C_{b}-C_{p}\right) Q}{\left(C_{b}+C_{p}\right)^{2}}\left\langle V_{b}\right\rangle+\frac{Q^{2}}{\left(C_{b}+C_{p}\right)^{2}}\right] \\
=-2 C_{p}\left\langle V_{b}\right\rangle^{2}+Q\left\langle V_{b}\right\rangle+\frac{Q^{2}}{2 C_{b}} \quad 0 \leq\left\langle V_{b}\right\rangle \leq \frac{Q}{2 C_{p}}
\end{gathered}
$$

The current in the inductor peaks when $V_{p}(t)=V_{b}(t)$, and can be calculated from Equation (4.25):

$$
\langle i\rangle=i(u)=i\left(\frac{\pi}{2 \omega}\right)=\frac{\left\langle V_{p}\right\rangle-\left\langle V_{b}\right\rangle}{L \omega} e^{-\left(\frac{\alpha \pi}{2 \omega}\right)}
$$


where $\alpha=\frac{R}{2 L}, \omega=\sqrt{\frac{1}{L C_{p}}}$, and the energy stored in the inductor is:

$$
\begin{gathered}
E_{L}=\frac{1}{2} L\langle i\rangle^{2}=\frac{1}{2} \frac{\left(\left\langle V_{p}\right\rangle-\left\langle V_{b}\right\rangle\right)^{2}}{L \omega^{2}} e^{-\left(\frac{\alpha \pi}{\omega}\right)} \\
E_{L}=\frac{1}{2} \frac{\left(\frac{Q}{C_{p}}-2\left\langle V_{b}\right\rangle\right)^{2}}{\frac{1}{C_{p}}} \gamma^{\prime} \quad 0 \leq\left\langle V_{b}\right\rangle \leq \frac{Q}{2 C_{p}}
\end{gathered}
$$

where $\gamma^{\prime}=e^{-\frac{\alpha \pi}{\omega}}$.

In the second time interval, as the switch is open, the electric circuit model during the second charging process is a second order RLC circuit, shown in Figure 4.18. The energy left in the inductor is transferred to $C_{b}$ through the freewheeling diode until the current in it drops from $\langle i\rangle$ to 0 , and a part of the energy is lost due to the inductor internal resistor $R$ and electromagnetic loss.

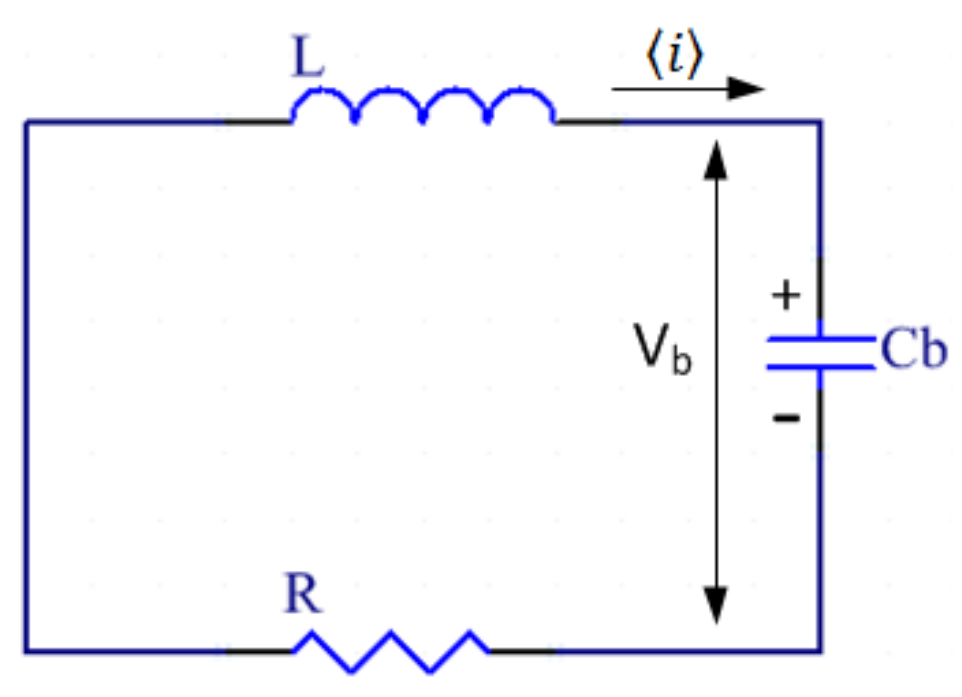

Figure 4.18 Second order RLC circuit. 
The energy stored in the bucket capacitor during the second time interval is:

$$
E_{2}=A \cdot E_{L}=\frac{C_{p}}{2}\left(\frac{Q}{C_{p}}-2\left\langle V_{b}\right\rangle\right)^{2} A \gamma^{\prime} \quad 0 \leq\left\langle V_{b}\right\rangle \leq \frac{Q}{2 C_{p}}
$$

where $A=\frac{E_{2}}{E_{L}}$ is the percentage of the inductor energy transferred to the bucket capacitor.

So the overall stored energy during compression is:

$$
E_{c o m}=E_{1}+E_{2}=-2 C_{p}\left\langle V_{b}\right\rangle^{2}+Q\left\langle V_{b}\right\rangle+\frac{Q^{2}}{2 C_{b}}+\frac{C_{p}}{2}\left(\frac{Q}{C_{p}}-2\left\langle V_{b}\right\rangle\right)^{2} A \gamma^{\prime}
$$

The total generated energy $E$ of each force cycle should be the total energy stored during compression and release.

$$
\begin{gathered}
E=E_{\text {com }}+E_{\text {rel }}=2 E_{\text {com }} \\
=-4 C_{p}\left\langle V_{b}\right\rangle^{2}+2 Q\left\langle V_{b}\right\rangle+\frac{Q^{2}}{C_{b}}+C_{p}\left(\frac{Q}{C_{p}}-2\left\langle V_{b}\right\rangle\right)^{2} A \gamma^{\prime} \\
=-4 C_{p}\left\langle V_{b}\right\rangle^{2}+2 Q\left\langle V_{b}\right\rangle+\frac{Q^{2}}{C_{b}}+B C_{p}\left(2\left\langle V_{b}\right\rangle-\frac{Q}{C_{p}}\right)^{2} \\
=4(B-1) C_{p}\left\langle V_{b}\right\rangle^{2}+2(1-2 B) Q\left\langle V_{b}\right\rangle+\frac{Q^{2}}{C_{b}}+B \frac{Q^{2}}{C_{p}}
\end{gathered}
$$

where $B=A \gamma^{\prime}$ and $\mathrm{B} \in(0,1)$.

As shown in Figure 4.19, Equation (4.57) is quadratic, and the maximum voltage the bucket capacitor can be rectified to is:

$$
\left\langle V_{b}\right\rangle_{\max }=\frac{Q}{2 C_{p}}
$$

which is half of the open circuit piezoelectric voltage. To maximize the energy increment $E$, the rectified voltage is related to B. As $\left\langle V_{b}\right\rangle_{o p t} \geq 0$, 


$$
\left\langle V_{b}\right\rangle_{o p t}=\left\{\begin{array}{cc}
\frac{(2 B-1) Q}{4(B-1) C_{p}} & 0<B \leq \frac{1}{2} \\
0 & \frac{1}{2}<B<1
\end{array}\right.
$$

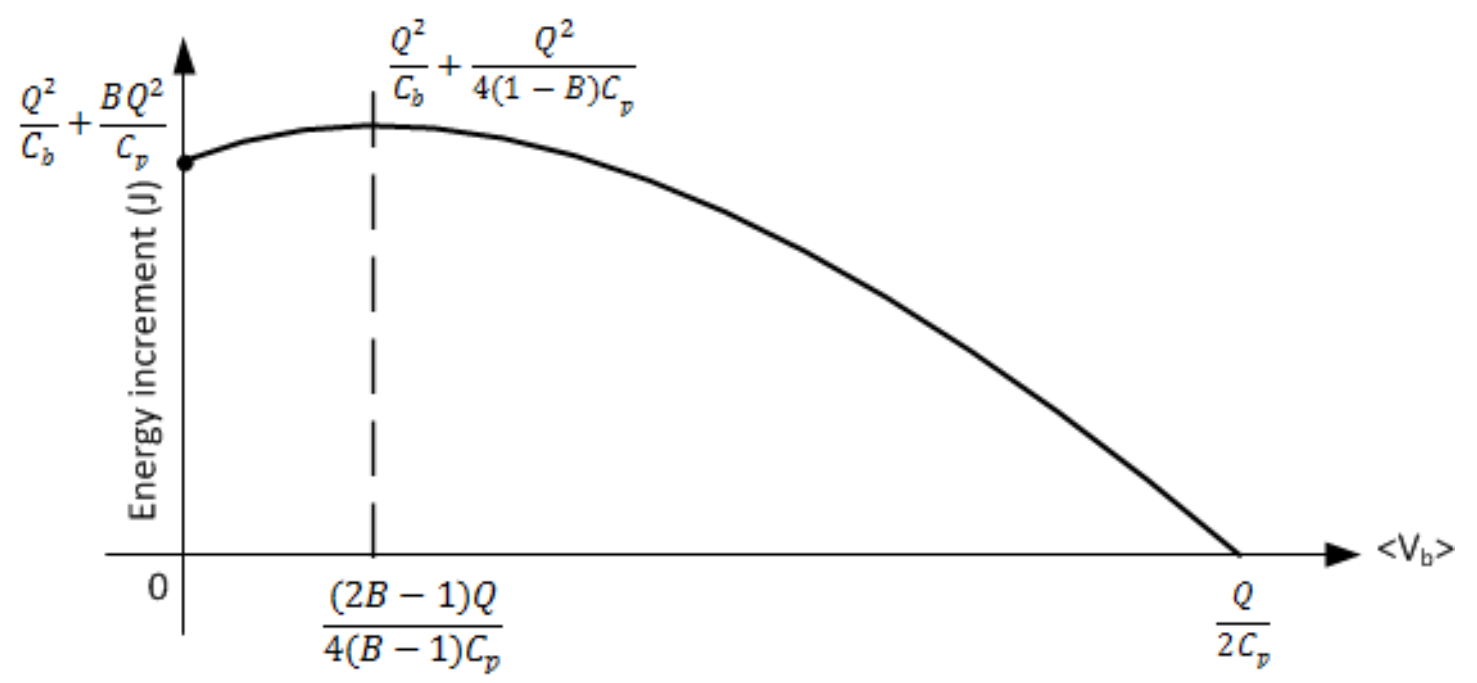

Figure 4.19 Energy increment in $C_{b}$ according to the rectified voltage $\left\langle V_{b}>\right.$.

Thus, the bucket capacitor voltage should be rectified to $\left\langle V_{b}\right\rangle_{o p t}$, and the corresponding maximum energy increment in the bucket capacitor $C_{b}$ during the force cycle is:

$$
E_{\max }=\left\{\begin{array}{cc}
\frac{Q^{2}}{C_{b}}+\frac{Q^{2}}{4(1-B) C_{p}} & 0<B \leq \frac{1}{2} \\
\frac{Q^{2}}{C_{b}}+\frac{B Q^{2}}{C_{p}} & \frac{1}{2} \leq B<1
\end{array}\right.
$$

From Equation (4.59) and (4.60), the optimal rectified voltage $V_{b}$ and the maximum stored energy $E_{\max }$ vary with $B$, as illustrated in Figure 4.20 . Assuming $C_{b} \gg C_{p}$, the item that includes $C_{b}$ in Equation (4.60) can be ignored. The optimal rectified voltage $V_{b}$ keeps decreasing from $0.25 \frac{Q}{C_{p}}$ to 0 when $B$ is between 0 and 0.5 , and the maximum 
energy increment $E_{\text {max }}$ increases from $0.25 \frac{Q^{2}}{C_{p}}$ to $0.5 \frac{Q^{2}}{C_{p}}$. When $B$ is between 0.5 and 1 , $V_{b}$ is kept at 0 , and $E_{\text {max }}$ keeps increasing from $0.5 \frac{Q^{2}}{C_{p}}$ to $\frac{Q^{2}}{C_{p}}$.

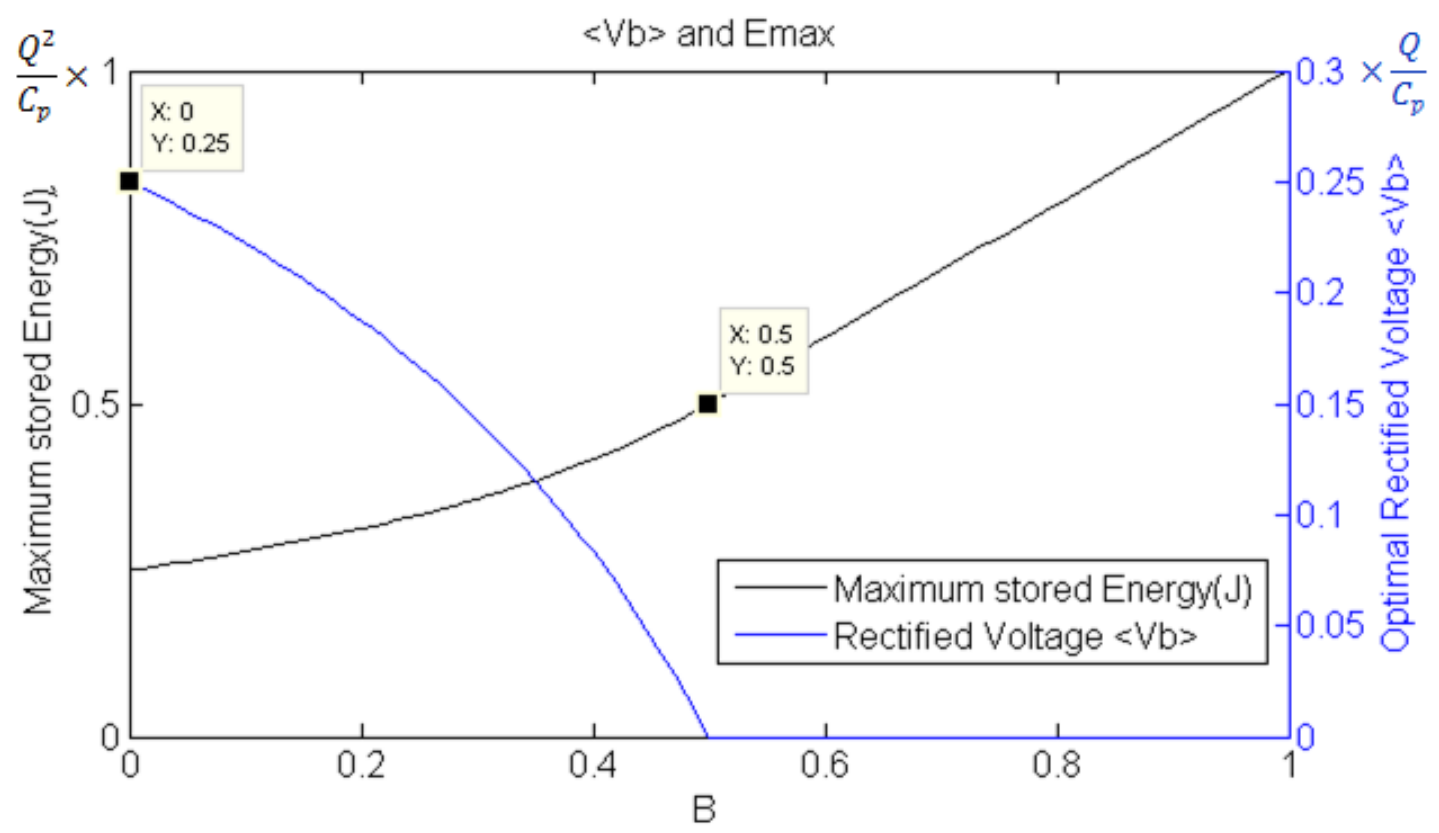

Figure 4.20 $<V_{b}>$ and $E_{\max }$ depending on $B$.

\subsubsection{Synchronized Electric Charge Extraction (SECE) topology}

The Synchronous Electric Charge Extraction (SECE) topology [81, 82] is derived from the Synchronized Switch Damping (SSD) technique [83, 84], which shunts the piezoelectric devices through an inductor synchronously with the mechanical load and can be applied in vibration damping and noise reduction systems. The equivalent electric circuit of the SECE topology is shown in Figure 4.21. 


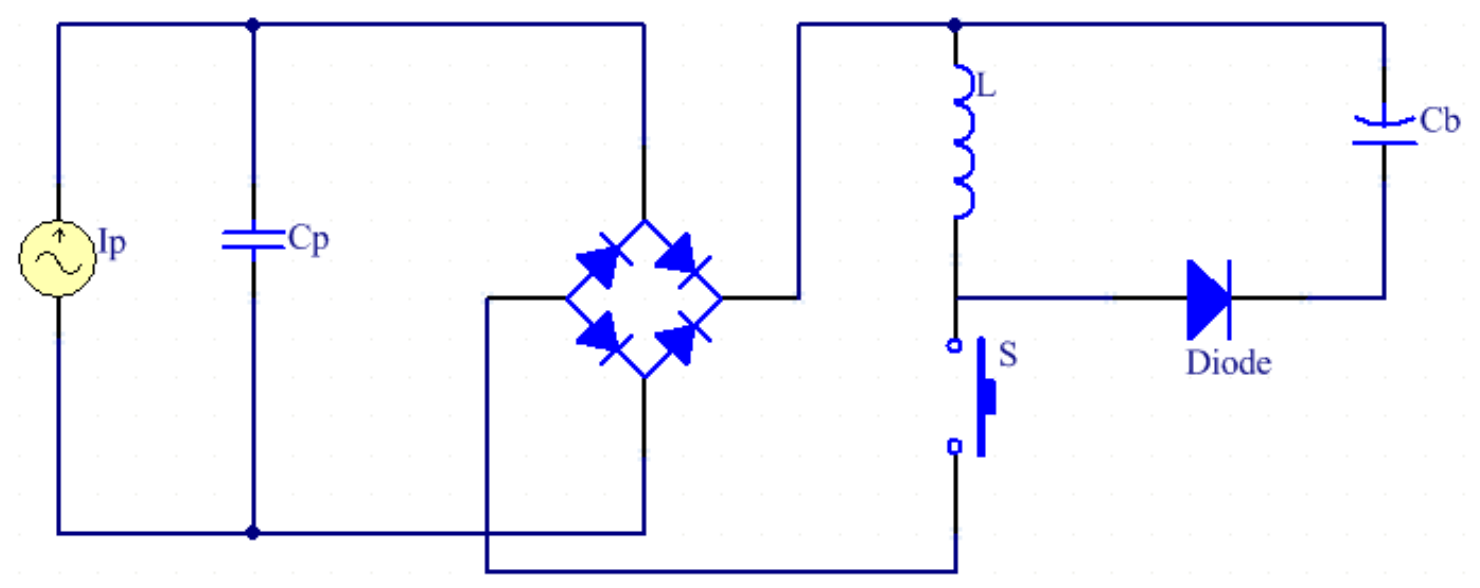

Figure 4.21 Equivalent electric circuit of the SECE topology.

The SECE topology is very similar to the SSDCI topology, and it discharges the piezoelectric capacitor $C_{p}$ if the piezoelectric current peaks. The power conditioning process has two steps. In the first step, the switch turns on when the piezoelectric current peaks, and the piezoelectric capacitor $C_{p}$ is discharged through the inductor. In the second step, when the piezoelectric voltage $V_{p}$ drops to zero, the switch turns off, and then the energy stored in the inductor transfers into the bucket capacitor $C_{b}$. Figure 4.22 illustrates the waveforms of the SECE topology, compared with the SSDCI topology, the piezoelectric voltage drops to zero regardless of the value of the rectified voltage $\left\langle V_{b}\right\rangle$. 


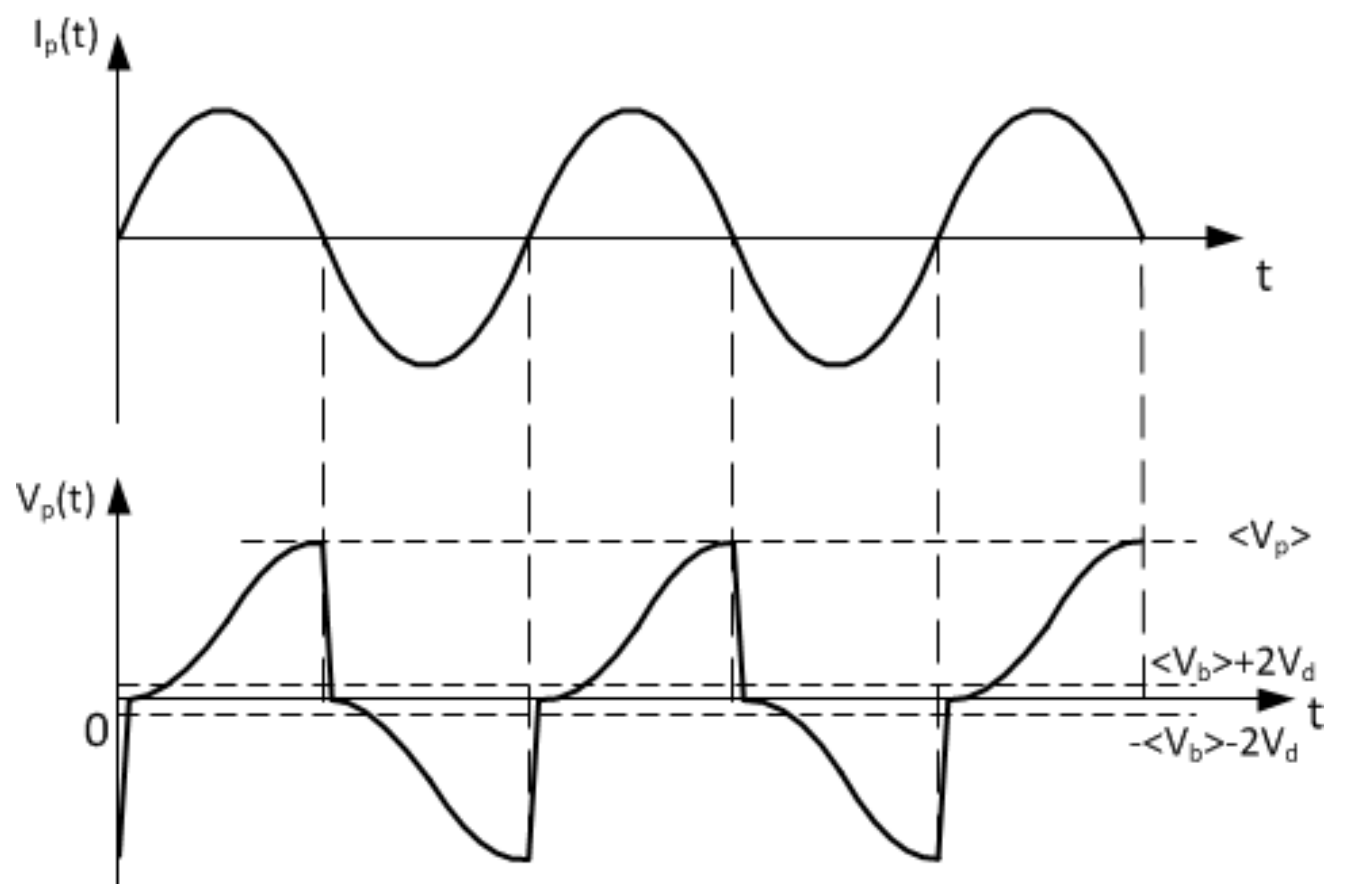

Figure 4.22 Waveforms of the SECE topology.

In the first power conditioning step, when the switch is turned on, a second order RLC circuit is established as drawn in Figure 4.10, with $C_{b}=\infty$. From Equation (4.27), the piezoelectric voltage $V_{p}(t)$ is:

$$
\begin{aligned}
V_{p}(t) & =\left\langle V_{p}\right\rangle e^{-\alpha t}\left[\cos (\omega t)+\frac{\mathrm{R}}{2 \omega L} \sin (\omega t)\right] \\
& =\left\langle V_{p}\right\rangle \beta e^{-\alpha t} \cos (\omega t-\phi)
\end{aligned}
$$

where $\omega=\sqrt{\frac{1}{L C_{p}}}, \beta=\sqrt{\frac{R^{2}}{4 \omega^{2} L^{2}}+1}, \phi=\tan ^{-1}\left(\frac{\mathrm{R}}{2 \omega L}\right)=\tan ^{-1}\left(\sqrt{\frac{R^{2} C_{p}}{4 L}}\right)$.

From Equation (4.25), the piezoelectric current $i(t)$ is:

$$
i(t)=\frac{\left\langle V_{p}\right\rangle}{L \omega} e^{-\alpha t} \sin (\omega t)
$$


Given that the piezoelectric open circuit voltage peaks at $\left\langle V_{p}\right\rangle$ and assuming that the switching process takes time $u$, the voltage of the piezoelectric capacitor should be zero when the switching process is stopped. According to Equation (4.61),

$$
V_{p}(u)=\left\langle V_{p}\right\rangle \beta e^{-\alpha u} \cos (\omega u-\phi)=0
$$

From Equation (4.47), we know $\phi \approx 0$, and therefore

$$
u=\frac{\pi+2 \phi}{2 \omega} \approx \frac{\pi}{2 \omega}
$$

According to Figure 4.22 , the piezoelectric capacitor $C_{p}$ is charged from zero with the generated charge $\mathrm{Q}$, and therefore the piezoelectric voltage peak $\left\langle V_{p}\right\rangle$ is:

$$
\left\langle V_{p}\right\rangle=\frac{Q}{C_{p}}
$$

The current in the inductor peaks when $V_{p}(t)=V_{b}(t)$, and can be calculated from Equation(4.62):

$$
\langle i\rangle=i(u)=i\left(\frac{\pi}{2 \omega}\right)=\frac{\left\langle V_{p}\right\rangle}{L \omega} e^{-\frac{\alpha \pi}{2 \omega}}
$$

where $\alpha=\frac{R}{2 L}, \omega=\sqrt{\frac{1}{L C_{p}}}$, and the energy stored in the inductor is:

$$
E_{L}=\frac{1}{2} L\langle i\rangle^{2}=\frac{\left\langle V_{p}\right\rangle^{2}}{2 L \omega^{2}} e^{-\frac{\alpha \pi}{\omega}}=\frac{Q^{2}}{2 C_{p}} \gamma^{\prime}
$$

where $\gamma^{\prime}=e^{-\frac{\alpha \pi}{\omega}}$.

In the second step of the power conditioning, the switch is turned off and the energy stored in the inductor $E_{L}$ transfers to the bucket capacitor $C_{b}$ as shown in Figure 4.18. The energy stored in the bucket capacitor $C_{b}$ during the compression is: 


$$
E=A \cdot E_{L}=\frac{Q^{2}}{2 C_{p}} A \gamma^{\prime}
$$

where $A=\frac{E}{E_{L}}$ is the percentage of the inductor energy transferred to the bucket capacitor.

So the overall stored energy during compression is:

$$
E_{c o m}=E=\frac{Q^{2}}{2 C_{p}} A \gamma^{\prime}
$$

The total generated energy $E$ of each force cycle should be the total energy stored during compression and release.

$$
E=E_{c o m}+E_{r e l}=2 E_{c o m}=\frac{Q^{2}}{C_{p}} A \gamma^{\prime}=B \frac{Q^{2}}{C_{p}}
$$

where $B=A \gamma^{\prime}$ and $B \in(0,1)$.

As shown in Figure 4.23, the energy increment is constant and independent from the rectified voltage $\left\langle V_{b}\right\rangle$, and the voltage the bucket capacitor $C_{b}$ can be rectified to is unlimited until the polling voltage of the piezoelectric material.

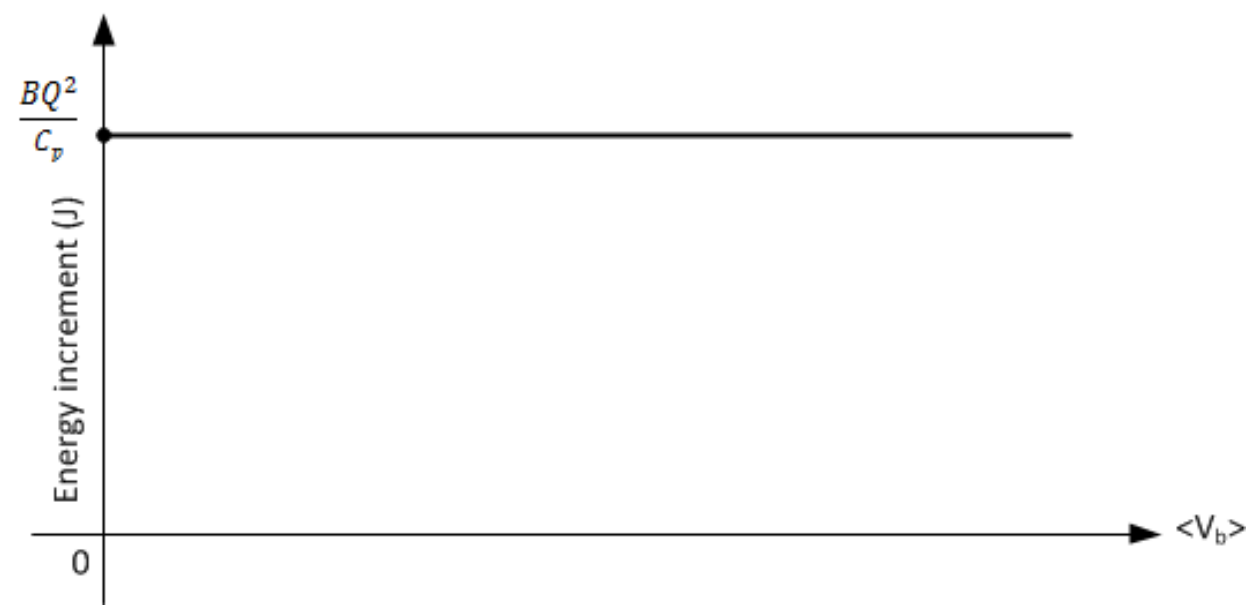

Figure 4.23 Energy increment in $C_{b}$ according to the rectified voltage $\left\langle V_{b}>\right.$. 


\subsubsection{Double Synchronized Switch Harvesting (DSSH) topology}

The Double Synchronized Switch Harvesting (DSSH) topology is a combination of the series SSHI and the SECE topology [85]. This topology has two switches, S1 and S2, as shown in Figure 4.24.

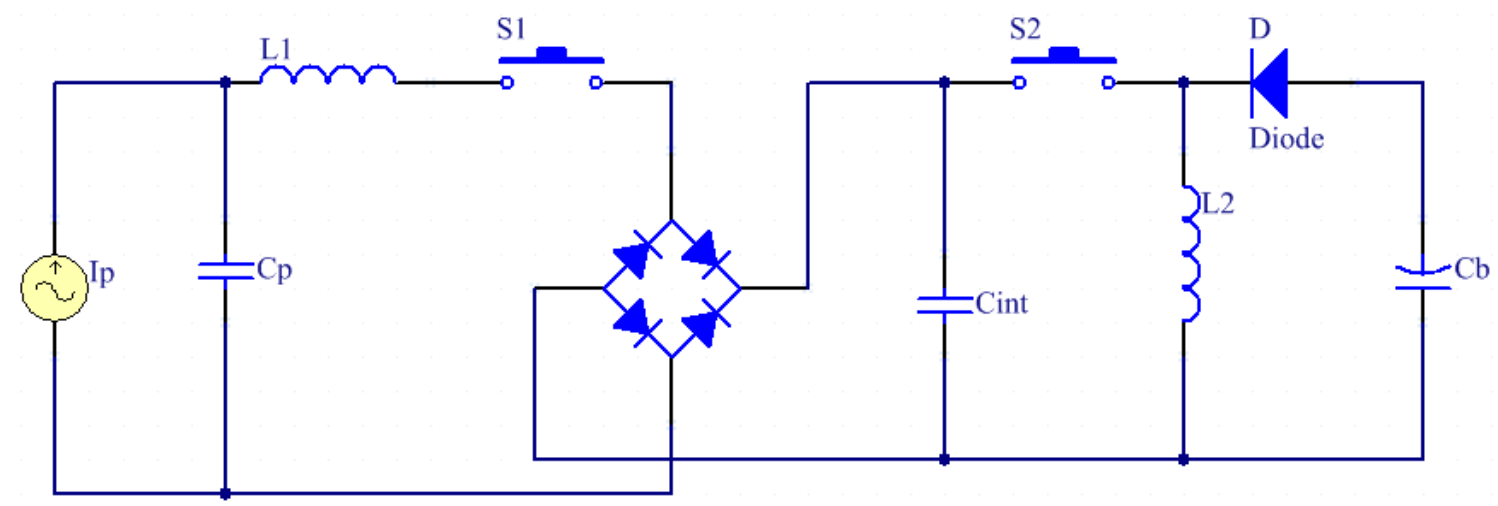

Figure 4.24 Equivalent electric circuit of the DSSH topology.

The power conditioning process of the DSSH topology is separated into three steps. In the first step, the DSSH topology works just like the series SSHI topology. When the piezoelectric current peaks, switch S1 is turned on, and then a part of the piezoelectric energy transfers into the intermediate capacitor $C_{i n t}$, and the remaining energy is used in the $C_{p}$ voltage inversion process. From the second step, the DSSH topology works just like the SEVE topology. The switch S1 is turned off, and the switch S2 is turned on. The energy stored in $C_{\text {int }}$ keeps flowing into the inductor $L_{2}$ until the voltage of $C_{\text {int }}$ drops to zero, and then switch $\mathrm{S} 2$ is turned off. In the next step, the energy stored in the inductor $L_{2}$ transfers into the bucket capacitor $C_{b}$ through a freewheeling diode. The waveforms of the DSSH topology is the same as that of the series SSHI topology as illustrated in Figure 4.9. 
Because the first power conditioning step of the DSSH topology is exactly the same as the series SSHI topology, from Equation (4.30) the voltage of $C_{p}$ and $C_{\text {int }}$ in the end of this step should be:

$$
\left\{\begin{array}{l}
V_{p}\left(\frac{\pi}{\omega_{1}}\right)=\frac{C_{p}}{C_{i n t}+C_{p}}\left\langle V_{p}\right\rangle+\frac{C_{i n t}}{C_{p}+C_{i n t}}\left\langle V_{i n t}\right\rangle-\frac{C_{i n t}}{C_{i n t}+C_{p}}\left(\left\langle V_{p}\right\rangle-\left\langle V_{i n t}\right\rangle\right) \gamma_{1} \\
V_{i n t}\left(\frac{\pi}{\omega_{1}}\right)=\frac{C_{p}}{C_{i n t}+C_{p}}\left\langle V_{p}\right\rangle+\frac{C_{i n t}}{C_{p}+C_{i n t}}\left\langle V_{i n t}\right\rangle+\frac{C_{p}}{C_{i n t}+C_{p}}\left(\left\langle V_{p}\right\rangle-\left\langle V_{i n t}\right\rangle\right) \gamma_{1}
\end{array}\right.
$$

where $\omega_{1}=\sqrt{\frac{1}{L C_{s}}}, C_{s}=\frac{C_{p} C_{i n t}}{C_{p}+C_{i n t}}, \gamma_{1}=e^{-\frac{\alpha_{1} \pi}{\omega_{1}}}, \alpha_{1}=\frac{R_{1}}{2 L_{1}}$. Assuming the ratio of $C_{i n t}$ to $C_{p}$ is $n$, and $\left\langle V_{\text {int }}\right\rangle$ is zero, Equation (4.71) becomes:

$$
\left\{\begin{array}{l}
V_{p}\left(\frac{\pi}{\omega_{1}}\right)=\frac{1-n \gamma_{1}}{n+1}\left\langle V_{p}\right\rangle \\
V_{\text {int }}\left(\frac{\pi}{\omega_{1}}\right)=\frac{1+\gamma_{1}}{n+1}\left\langle V_{p}\right\rangle
\end{array}\right.
$$

According to Figure 4.9, $\left\langle V_{p}^{\prime}\right\rangle=V_{p}\left(\frac{\pi}{\omega_{1}}\right),\left\langle V_{p}\right\rangle=-\left\langle V_{p}^{\prime}\right\rangle+\frac{Q}{C_{p}}$, therefore

$$
\left\langle V_{p}\right\rangle=\frac{n+1}{n\left(1-\gamma_{1}\right)+2} \cdot \frac{Q}{C_{p}}
$$

The voltage of the intermediate capacitor $C_{i n t}$, and the piezoelectric capacitor $C_{p}$ in the end of the first steps becomes:

$$
\left\{\begin{array}{l}
V_{p}\left(\frac{\pi}{\omega_{1}}\right)=\frac{1-n \gamma_{1}}{n-n \gamma_{1}+2} \cdot \frac{Q}{C_{p}} \\
V_{\text {int }}\left(\frac{\pi}{\omega_{1}}\right)=\frac{1+\gamma_{1}}{n-n \gamma_{1}+2} \cdot \frac{Q}{C_{p}}
\end{array}\right.
$$


In the next two power conditioning steps, the DSSH topology works the same as the SECE topology, from Equation (4.66) and (4.73), the energy stored in the inductor $L_{2}$ in the second step is:

$$
E_{L_{2}}=\frac{1}{2} L_{2}\langle i\rangle^{2}=\frac{\left\langle V_{i n t}\right\rangle^{2}}{2 L \omega^{2}} e^{-\frac{\alpha_{2} \pi}{\omega_{2}}}=\frac{n\left(1+\gamma_{1}\right)^{2}}{2\left(n-n \gamma_{1}+2\right)^{2}} \cdot \frac{Q^{2}}{C_{p}} \gamma_{2}
$$

where $\alpha_{2}=\frac{R_{2}}{2 L_{2}}, \gamma_{2}=e^{-\frac{\alpha_{2} \pi}{\omega_{2}}}, \omega_{2}=\sqrt{\frac{1}{L_{2} C_{i n t}}}$. From Equation(4.68), the energy stored in the bucket capacitor $C_{b}$ during the compression is:

$$
E_{\text {com }}=A \cdot E_{L_{2}}=\frac{n\left(1+\gamma_{1}\right)^{2}}{2\left(n-n \gamma_{1}+2\right)^{2}} \cdot \frac{Q^{2}}{C_{p}} A \gamma_{2}
$$

where $A=\frac{E}{E_{L_{2}}}$ is the percentage of the inductor energy transferred to the bucket capacitor. The total generated energy $E$ of each force cycle should be the total energy stored during compression and release.

$$
\begin{gathered}
E=E_{c o m}+E_{r e l}=2 E_{c o m}=\frac{n\left(1+\gamma_{1}\right)^{2}}{\left(n-n \gamma_{1}+2\right)^{2}} \cdot \frac{Q^{2}}{C_{p}} A \gamma_{2} \\
=\frac{n\left(1+\gamma_{1}\right)^{2}}{\left(n-n \gamma_{1}+2\right)^{2}} B \frac{Q^{2}}{C_{p}}
\end{gathered}
$$

where $B=A \gamma_{2}$ and $B \in(0,1)$. By cancelling the derivative of the energy $E$ to the ratio $n$, the optimal ratio is:

$$
n_{\text {opt }}=\frac{2}{1-\gamma_{1}}
$$

Accordingly, the optimal energy increment $E_{\text {opt }}$ is:

$$
E_{\text {opt }}=\frac{\left(1+\gamma_{1}\right)^{2}}{8\left(1-\gamma_{1}\right)} B \frac{Q^{2}}{C_{p}}
$$


From Equation (4.73), the according piezoelectric voltage peak is:

$$
\left\langle V_{p}\right\rangle_{o p t}=\frac{3-\gamma_{1}}{4\left(1-\gamma_{1}\right)} \cdot \frac{Q}{C_{p}}
$$

As shown in Figure 4.23, for the DSSH topology with the optimal ratio $n$, the energy increment of $C_{b}$ during each load is constant and independent from the rectified voltage $\left\langle V_{b}\right\rangle$, and the voltage the bucket capacitor $C_{b}$ can be rectified to is unlimited until the polling voltage of the piezoelectric material.

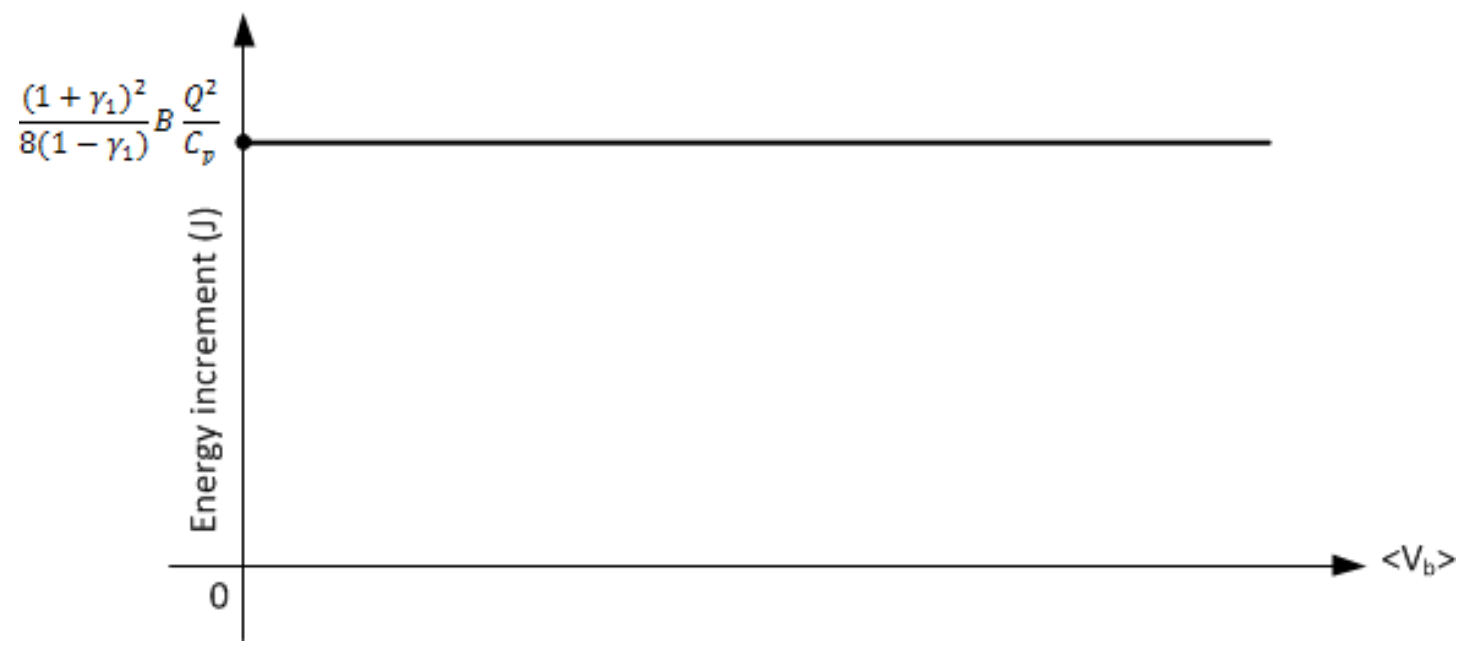

Figure 4.25 Energy increment in $C_{b}$ according to the rectified voltage $<V_{b}>$.

\subsection{Selected Electric circuit topology}

Recent different piezoelectric KEH topologies are studied in the previous sections. Their performance in terms of energy increment during each load period needs to be compared for the purpose of choosing the most suitable one for this project. 
As stated earlier, the piezoelectric energy is finally stored in a bucket capacitor $C_{b}$ and the energy increment is related to the rectified voltage $\left\langle V_{b}\right\rangle$. Figure 4.26 summarizes the relationship between the energy increment in one period and the corresponding rectified voltage $\left\langle V_{b}\right\rangle$ for these seven different topologies. It is clear that although the full-bridge and half-bridge direct charging topologies are the simplest, their maximum output energy are the lowest due to the energy waste on discharging $C_{p}$.

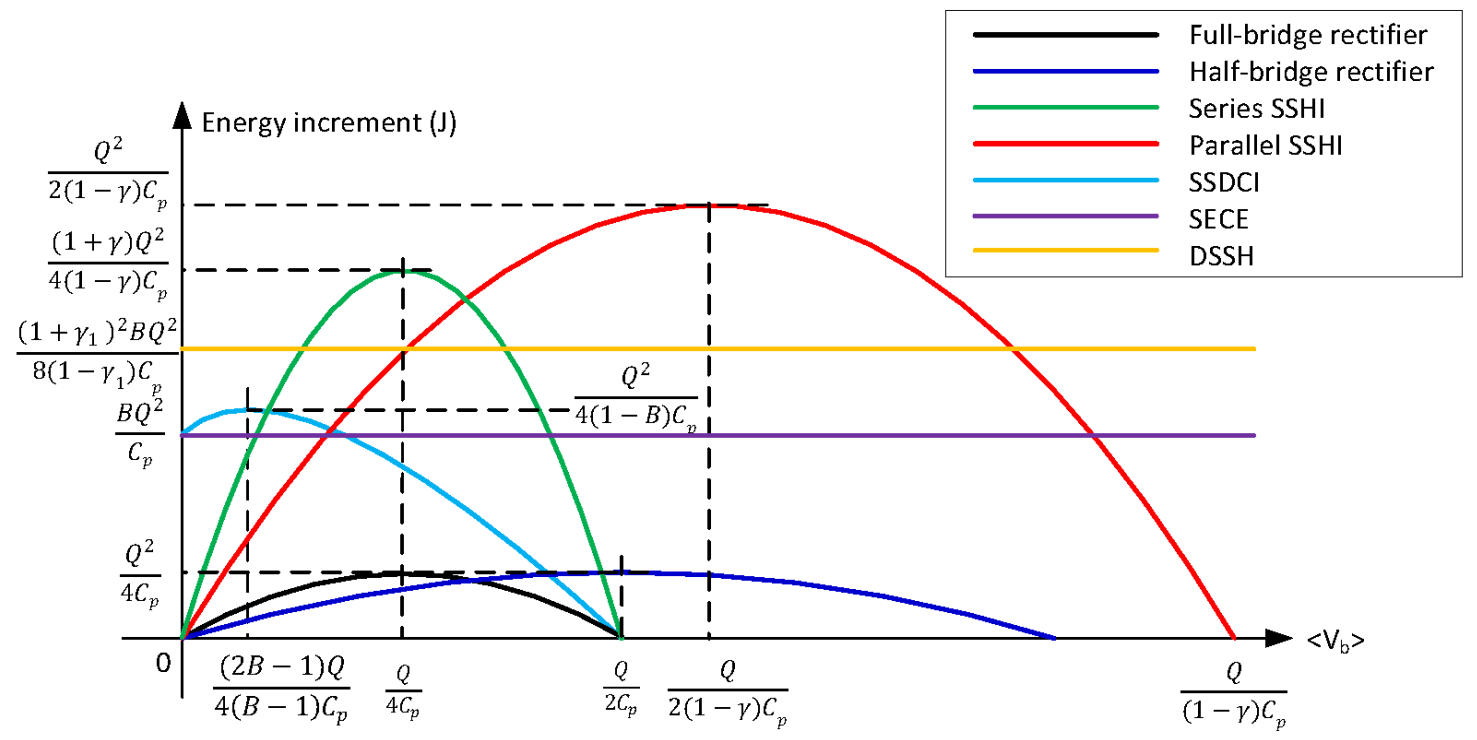

Figure 4.26 The energy increment of different topologies during each load period with various rectified voltage $\left\langle V_{b}\right\rangle$.

The parallel SSHI has the potential to generate the most energy. From Equation (4.45), the higher $\gamma$ is, the higher the maximum generated energy, but the optimal rectified voltage $\left\langle V_{b}\right\rangle_{o p t}$ will be higher as well according to Equation (4.44). The energy generated by the series SSHI is relatively high and also depends on $\gamma$ according to Equation (4.36). Its optimal rectified voltage is constant (from Equation (4.35)), but according to Equation 
(4.32), the piezoelectric voltage peak $\left\langle V_{p}\right\rangle$ will be much higher than the rectified voltage $\left\langle V_{b}\right\rangle$, and is prone to be over the piezoelectric voltage limit (i.e. its original poling voltage). In addition, like the direct charge topology and the parallel SSHI, the Series SSHI only generates the most energy when the rectified voltage is at its optimal value, which is over one quarter of the piezoelectric open circuit voltage and could be much higher than the desired low rectified voltage typically between $3 \mathrm{~V}$ and $5 \mathrm{~V}$.

The SSDCI topology is able to generate moderate energy when the rectified voltage is optimal, and the energy increases with $B$. As already described in Figure 4.20, the increase of the value $B$ will make $\left\langle V_{b}\right\rangle_{\text {opt }}$ closer to zero, and if $B>0.5,\left\langle V_{b}\right\rangle_{o p t}$ will become zero. Compared with the SECE topology, it has the same energy increment if the rectified voltage $\left\langle V_{b}\right\rangle$ is zero. If $B<0.5$, the SSDCI will have a higher maximum energy increment. The advantage of the SECE topology is that its energy increment is constant and independent of the rectified voltage. However, its drawback is that it requires very precise switch control. If the switch is turned off a little later, the energy in the inductor will dissipate through two diodes in the diode bridge (Figure 4.21). For the SSDCI topology, the energy in the inductor can only transfer to the bucket capacitor $C_{b}$ either through the freewheeling diode or the diodes in the diode bridge (Figure 4.16). The DSSH has the advantage of the SECE topology that its energy increment is constant and independent of the rectified voltage, and it has the potential of having a higher optimal energy increment than SECE if $\gamma_{1}>0.66$. It also bring the drawbacks of the both the series SSHI topology and the SECE topology. Like the series SSHI topology, the piezoelectric voltage peak $\left\langle V_{p}\right\rangle$ depends on the initial piezoelectric voltage $\left\langle V_{p}^{\prime}\right\rangle$ from 
Figure 4.9. Under the random excitations from the vehicle wheels, $\left\langle V_{p}^{\prime}\right\rangle$ needs to be maintained at the end of each load in order to get the maximum energy, which increases the circuit complexity. The DSSH topology also has to control two switches precisely, which is required for the voltage inversion in the first step and the energy transfer in the second step.

Hence, when the rectified voltage is kept low (3 to $5 \mathrm{~V}$ ), the SSDCI topology is able to generate more energy than the other topologies except the DSSH topology. Considering the switch control simplicity, the SSDCI topology will be chosen as the piezoelectric KEH topology for the piezoelectric energy source in this project. 


\section{Chapter: Energy Harvesting Electric System}

The following sections describe the circuit design for piezoelectric energy harvesting based on the SSDCI topology. In order to use this circuit topology to harvest energy independently, the circuit configuration improvement includes a peak detection circuit and an auxiliary circuit to shunt a part of the piezoelectric energy to control the switch. The designed harvesting circuit is tested with both the KEH container under a hydraulic load and the KEH speed bump under a test vehicle.

\subsection{Power Harvesting Circuit Design Considerations}

Firstly, as stated in Chapter 4, the switch in the SSDCI topology is kept open (Figure 4.16) until the generated piezoelectric current changes direction and the piezoelectric voltage peaks. Whenever there is a current peak, the switch is closed and the bucket capacitor is able to get electric charge from the piezoelectric source through an inductor. Therefore a circuit with zero-slope voltage peak detection function is required. The detection circuit should have an appropriate input impedance which shunts enough current to supply detectable logic signals to the CMOS logic components. The input impedance should not be too low, otherwise it will shunt too much current and affect the piezoelectric voltage peak (i.e., the available energy).

Secondly, because the piezoelectric KEHS is independent and possesses no external power supply other than the piezoelectric material, an ancillary circuit is required to shunt 
current from the piezoelectric transducer to initialize the system, and to supply power to the peak detection circuit.

Thirdly, for the stored energy in the bucket capacitor to be applied on the load, a DC-DC voltage regulator (such as a boost converter or a step-down converter) is necessary to boost or reduce the output voltage to a desired and stable level. Depending on the required duty cycle for the load, an ON/OFF load control with voltage hysteresis for the bucket capacitor should be considered.

In summary, the piezoelectric KEH circuit that implements the SSDCI topology should be able to meet the following requirements: (a) Detecting piezoelectric voltage peaks; (b) Shunting a small part of energy to power the peak detection circuit; (c) Automatically switching the load ON/OFF with voltage hysteresis for the bucket capacitor.

\subsection{Electronic Circuit Design}

This section describes the design of the piezoelectric KEH circuit, as shown in Figure 5.1, and the designed circuit intends to provide the following sequential functions: (1) Piezoelectric voltage rectification and "cold start"; (2) Zero-slope peak detection and switch control; (3) Load switch ON/OFF control with voltage hysteresis. 


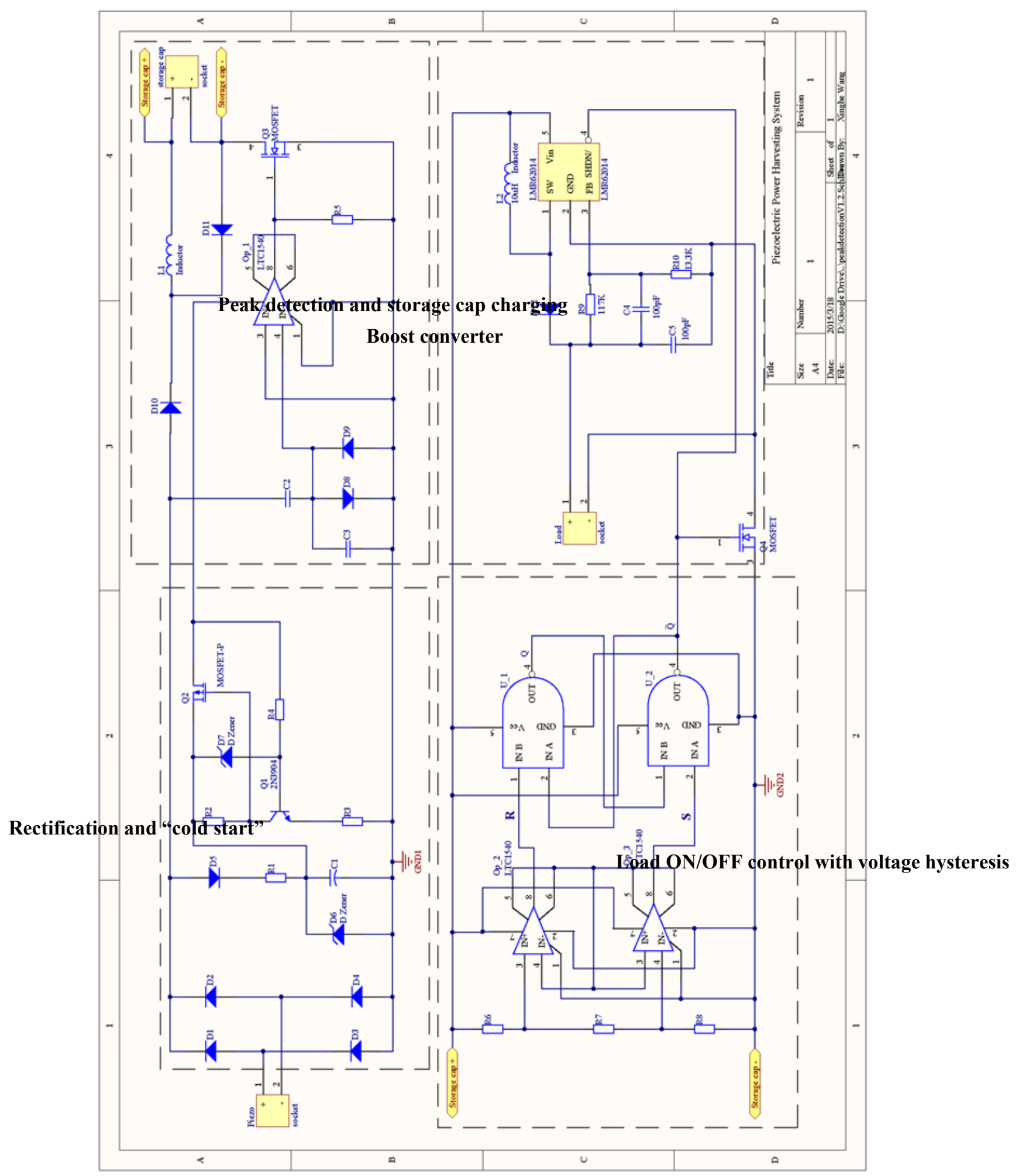

Figure 5.1 Piezoelectric energy harvesting circuit. 


\subsubsection{Piezoelectric voltage rectification and "cold start"}

The piezoelectric voltage is fully rectified by the diode bridge D1-D4. Then the resistor $\mathrm{R} 1$ shunts current to the capacitor $\mathrm{C} 1$, which supplies power for the peak detection circuit. The diode D5 prevents current leaking from the capacitor $\mathrm{C} 1$ to the capacitors in the peak detection circuit ( $\mathrm{C} 2$ and $\mathrm{C} 3$ ), and the diode $\mathrm{D} 6$ prevents $\mathrm{C} 1$ from being charged over the maximum input voltage of the operational comparator Linear Technology LTC 1540. The LTC 1540 is an ultralow power, single comparator with built-in reference. It was chosen because it has a wide supply range from $2 \mathrm{~V}$ to $11 \mathrm{~V}$, and an ultralow quiescent current (typically $0.3 \mu \mathrm{A}$ ). In order to ensure that the peak detection circuit capacitors and the capacitor $\mathrm{C} 1$ are charged simultaneously, the grounds of the "cold start" circuit and the peak detection circuit are connected together. The "cold start" circuit that controls Q1 and Q2 is inspired by the latching circuit used by the MIT Media Laboratory [31]. If the voltage of $\mathrm{C} 1$ is below a voltage threshold $V_{1}$, which is the sum of the breakdown voltage of the Zener diode D7 and the voltage drop $0.7 \mathrm{~V}$ between the Base and Emitter of the NPN transistor Q1 as described in Equation (5.1), both Q1 and Q2 are off and C1 experiences very high input impedance that minimizes the energy dissipation. As soon as $\mathrm{C} 1$ is charged over $V_{1}, \mathrm{Q} 1$ is turned on, and the P-Channel MOSFET Q2 is activated by the voltage across R2. Then the current starts to flow through R4 instead of D6, which in turn latches Q1. As Q2 is activated, C1 is able to supply energy for the following zeroslope peak detection circuit. When $\mathrm{C} 1$ is discharged below another voltage threshold $V_{2}$,

which is determined by R2 and R3, and the minimum gate threshold voltage $V_{G S(\min )}$ of Q2 as shown in Equation (5.1), Q2 is deactivated and so is Q1. Then the voltage of C1 stays just below V1, and waits for the next current pulse. 


$$
\left\{\begin{array}{c}
V_{1}=0.7+V_{D 7} \\
V_{2}=V_{G S(\text { min })} \times \frac{R_{2}+R_{3}}{R_{2}}
\end{array}\right.
$$

Compared with the MIT circuit design described in Appendix C, this "cold start" circuit design is able to power the peak detection circuit from the very beginning (i.e., when the rectified voltage is $0 \mathrm{~V}$ ), which increases the circuit efficiency.

\subsubsection{Zero-slope peak detection and switch control}

In the peak detection circuit, the zero-slope voltage peak signal is produced by a capacitor C3 in parallel with two diodes, D8 and D9, which are connected end-to-end, and is sensed by the operational amplifier (LTC 1540). The diodes limit the voltage of C3 to within the diode voltage drop (around $0.7 \mathrm{~V}$ ) in both directions. When the piezoelectric voltage $V_{p}$ is rising, D7 is forward biased and its voltage drop keeps the $I N^{-}$pin higher than the $I N^{+}$pin, and the output holds MOSFET Q3 deactivated. As soon as the piezoelectric voltage reaches peak value and begins to drop, D8 becomes forward biased and its voltage drop keeps the $I N^{+}$pin higher than the $I N^{-}$pin, and the output activates Q3. Then the energy stored in the piezoelectric capacitor is able to flow into the bucket capacitor $C_{b}$ through the inductor L1 until $V_{p}$ equals the rectified voltage. The energy left in L1 keeps charging the bucket capacitor through the freewheeling diode D11 as the SSDCI topology. The diode D10 prevents the charges in the bucket capacitor from feeding back to $\mathrm{C} 2$ and $\mathrm{C} 3$ through the body diodes of the power MOSFET Q3. 


\subsubsection{Load switch ON/OFF control with hysteresis}

In the case that the load consumes more power than the piezoelectric unit, the voltage hysteresis of $C_{b}$ allows the load to work in a low duty cycle condition. R6, R7, and R8 constitute a high-impedance voltage divider. Two comparators, Op_2 and Op_3, are connected with the voltage divider. The IN- pin of $\mathrm{Op}_{-} 2$ and the IN+ pin of $\mathrm{Op}_{-} 3$ are connected together to the reference pin. In this way, the order of $V_{A}$ (the voltage of point A), $V_{B}$ (the voltage of point B) and the reference voltage $V_{r e f}$ determines the comparators' output $R$ and $S$, which are also the input of the NAND set/reset latch. The truth table for the comparators and the NAND latch is shown in Table 5-1. The output of the NAND latch $\overline{\mathrm{Q}}$ controls the SHDN/ pin of the step-up voltage regulator and the N-channel MOSFET Q4 for true shutdown that prevents the load or the resistor R9 and R10 from draining current through L2 and D12.

Table 5-1 Truth table for the comparators and the NAND latch

\begin{tabular}{|c|c|c|c|c|c|}
\hline $\mathrm{IN}+$ & $\mathrm{IN}-$ & $\mathrm{R}$ & $\mathrm{S}$ & $\mathrm{Q}$ & $\overline{\mathrm{Q}}$ \\
\hline 0 & 0 & 0 & 1 & 1 & 0 \\
\hline 1 & 0 & 1 & 1 & $\mathrm{Q}$ & $\overline{\mathrm{Q}}$ \\
\hline 1 & 1 & 1 & 0 & 0 & 1 \\
\hline & & 0 & 0 & 0 & 1 \\
\hline
\end{tabular}

In order to keep the rectified voltage $\left\langle V_{b}\right\rangle$ in the range between $V_{1}{ }^{\prime}$ and $V_{2}{ }^{\prime}$. The resistance of the voltage divider can be determined by:

$$
\left\{\begin{array}{l}
\frac{R 7+R 8}{R 6+R 7+R 8}=\frac{V_{r e f}}{V_{1}{ }^{\prime}} \\
\frac{R 8}{R 6+R 7+R 8}=\frac{V_{r e f}}{V_{2}{ }^{\prime}}
\end{array}\right.
$$




\subsection{Components Selections}

This section gives the selections of both the active components (Table 5-2) and the passive components (Table 5-3) in the power harvesting electric circuit, as well as the rationale for these selections.

Table 5-2 Active Components

\begin{tabular}{|c|c|c|}
\hline \multicolumn{3}{|c|}{ Active Components } \\
\hline Designation & Description & Name (Mfg.) \\
\hline Op_1-Op_3 & $\begin{array}{c}\text { Nanopower Comparator with } \\
\text { reference }\end{array}$ & LTC1540 (LTC) \\
\hline U_1-U_2 & 2-Input NAND Gate & Tc7SH00F (TOSHIBA) \\
\hline U_3 & Step-Up Voltage Regulator & LMR62014 (Texas Int.) \\
\hline
\end{tabular}

Table 5-3 Passive Components

\begin{tabular}{|c|c|c|c|}
\hline \multicolumn{4}{|c|}{ Passive Components } \\
\hline Designation & Value & Name (Mfg.) & Misc. Information \\
\hline $\begin{array}{l}\text { D1-D4, D5, } \\
\text { D8-D12 }\end{array}$ & & S1J (FAIRCHILD) & $\mathrm{V}_{\mathrm{RRM}}=600 \mathrm{~V}$ \\
\hline $\mathrm{R} 1$ & $3 \mathrm{M} \Omega$ & & $(+/-1 \%)$ \\
\hline R2 & $1 \mathrm{M} \Omega$ & & $(+/-1 \%)$ \\
\hline R3 & $1.5 \mathrm{M} \Omega$ & & $(+/-1 \%)$ \\
\hline $\mathrm{R} 4$ & $5 \mathrm{M} \Omega$ & & $(+/-5 \%)$ \\
\hline $\mathrm{C} 1$ & $0.1 \mu \mathrm{F}$ & & Aluminum \\
\hline D6 & $10 \mathrm{~V}$ & & Zener diode \\
\hline D7 & $9 \mathrm{~V}$ & & Zener diode \\
\hline Q1 & & 2N3904 (MCC) & NPN transistor \\
\hline $\mathrm{C} 2$ & $100 \mathrm{pF}$ & $\begin{array}{l}\text { 12068A101JAT2D } \\
\text { (AVX) }\end{array}$ & $\begin{array}{l}\text { Ceramic, }(+/-5 \%) \text {, } \\
\text { voltage rate: } 400 \mathrm{~V}\end{array}$ \\
\hline C3 & $20 \mathrm{pF}$ & & Ceramic, $(+/-5 \%)$ \\
\hline R5 & $2 \mathrm{M} \Omega$ & & $(+/-5 \%)$ \\
\hline L1 & $10 \mathrm{mH}$ & $\begin{array}{l}\text { 1140-103K-RC (Bourns } \\
\text { Inc.) }\end{array}$ & $\mathrm{I}_{\mathrm{sat}}=2.1 \mathrm{~A}, \mathrm{DCR}=2.76 \Omega$ \\
\hline Q2 & & $\begin{array}{c}\text { FQU5P20TU-ND } \\
\text { (FAIRCHILD) }\end{array}$ & $\begin{array}{c}\text { P-Channel MOSFET, } \\
\mathrm{V}_{\mathrm{DS}}=200 \mathrm{~V} \mathrm{~V}_{\mathrm{GS}(\mathrm{th})} \in(3,5)\end{array}$ \\
\hline Q3, Q4 & & STN3N45K3 (STM.) & $\begin{array}{c}\text { N-Channel MOSFET, } \\
\mathrm{V}_{\mathrm{DS}}=450 \mathrm{~V}, \mathrm{~V}_{\mathrm{GS}(\mathrm{th})} \in(3,5)\end{array}$ \\
\hline
\end{tabular}




\begin{tabular}{|c|c|c|c|}
\hline R6 & $3.83 \mathrm{M} \Omega$ & & $(+/-1 \%)$ \\
\hline R7 & $1 \mathrm{M} \Omega$ & & $(+/-1 \%)$ \\
\hline $\mathrm{R} 8$ & $1.5 \mathrm{M} \Omega$ & & $(+/-1 \%)$ \\
\hline $\mathrm{R} 9$ & $117 \mathrm{k} \Omega$ & & $(+/-1 \%)$ \\
\hline $\mathrm{R} 10$ & $13.3 \mathrm{k} \Omega$ & & $(+/-1 \%)$ \\
\hline $\mathrm{L} 2$ & $10 \mu \mathrm{F}$ & $\begin{array}{c}\text { VLF252010MT-100M } \\
\text { (TDK) }\end{array}$ & Ceramic \\
\hline $\mathrm{C} 4, \mathrm{C} 5$ & $100 \mathrm{pF}$ & & \\
\hline
\end{tabular}

In the voltage rectification and "cold start" circuit, the full-wave bridge that consists of four diodes D1, D2, D3 and D4 should have very high reverse voltage because the piezoelectric open circuit voltage peaks are hundreds of volts. Diode bridges which can tolerate a reverse voltage up to $600 \mathrm{~V}$ are chosen here. The N-channel MOSFETs used in this circuit needs to have a high maximum drain-source voltage $V_{D S}$, so STN3N45K3 from STMicroelectronics (STM.) is chosen as its $V_{D S}=450 \mathrm{~V}$. The aluminum capacitor $\mathrm{C} 1$ is the energy supplier for the comparator LTC 1540, which has a supply range from $2 \mathrm{~V}$ to $11 \mathrm{~V}$. Therefore a Zener diode with the breakdown voltage of $10 \mathrm{~V}$ is placed in parallel with the capacitor $\mathrm{C} 1$ so as to prevent $\mathrm{C} 1$ from being over charged. In addition, the voltage threshold $V_{1}$ of the latch circuit should below $10 \mathrm{~V}$. In order to make sure that the output of the comparator Op_1 is over the maximum gate threshold voltage $V_{G S(\max )}$ of Q3, the voltage threshold $V_{2}$ of the latch circuit is chosen to be $5 \mathrm{~V}$, which is the $V_{G S(\max )}$ of the MOSFET STM. STN3N45K3. According to Equation (5.1), given $V_{G S(\min )}=3 \mathrm{~V}$, the breakdown voltage of the Zener diode $\mathrm{D} 6$ is chosen to be $8.2 \mathrm{~V}$, and $\frac{R_{2}+R_{3}}{R_{2}}=\frac{5}{3}$. If $R_{2}+R_{3}=2.5 \mathrm{M} \Omega$, then $R_{2}=1.5 \mathrm{M} \Omega$ and $R_{3}=1 \mathrm{M} \Omega$. For the zero-slope peak detection circuit, the increase of $\mathrm{C} 2$ increases the shunt current and the charging speed of $\mathrm{C} 3$, while the decrease of $\mathrm{C} 3$ increases the charging speed of $\mathrm{C} 3 . \mathrm{C} 2$ and $\mathrm{C} 3$ are chosen to be $100 \mathrm{pF}$ and $20 \mathrm{pF}$ respectively. It should be noted that $\mathrm{C} 2$ should have a high 
voltage tolerance because it will have the same voltage as the piezoelectric voltage. The inductor L1 needs to be chosen very carefully because it should be ensured that the current through it does not exceed its saturation current. According to Equation (4.52), if $\frac{4 L}{C_{p} R^{2}} \gg 1$,

$$
\begin{aligned}
\langle i\rangle & =\frac{\left\langle V_{p}\right\rangle-\left\langle V_{b}\right\rangle}{L \omega} e^{-\left(\frac{\alpha \pi}{2 \omega}\right)}=\frac{\left\langle V_{p}\right\rangle-\left\langle V_{b}\right\rangle}{L \omega} e^{-\left(\frac{\pi}{2 \sqrt{\frac{4 L}{C_{p} R^{2}}}}\right)} \\
\approx & \frac{\left\langle V_{p}\right\rangle-\left\langle V_{b}\right\rangle}{L \omega}=\frac{\left\langle V_{p}\right\rangle-\left\langle V_{b}\right\rangle}{\sqrt{\frac{L}{C_{p}}-\frac{R^{2}}{4}}}
\end{aligned}
$$

From the open circuit voltage test on the KEH speed bump, the open circuit voltage peak $\left\langle V_{p}\right\rangle$ is $234 \mathrm{~V}$ and the piezoelectric capacitance $C_{p}=148 \mathrm{nF}$. Given the rectified voltage $\left\langle V_{b}\right\rangle$ is below $5 \mathrm{~V}$, which is far less than the voltage peak $\left\langle V_{p}\right\rangle,\left\langle V_{p}\right\rangle-\left\langle V_{b}\right\rangle$ is assumed to be $234 \mathrm{~V}$. The inductor 1140-103K-RC from Bourns Inc. was chosen because it has a large inductance of $10 \mathrm{mH}$ and a large saturation current of 2.1 A. The dissipation resistance of the inductor is $2.76 \Omega$. Therefore the current peak $i_{p}$ through this inductor can be calculated from Equation (5.3):

$$
i_{p}=\frac{\left\langle V_{p}\right\rangle-\left\langle V_{b}\right\rangle}{\sqrt{\frac{L}{C_{p}}-\frac{R^{2}}{4}}}=\frac{234}{\sqrt{\frac{0.01}{1.48 \times 10^{-7}}-\frac{2.76^{2}}{4}}} \approx 0.9 \mathrm{~A}
$$

which is below the saturation current of the inductor. 
Assuming the rectified voltage $\left\langle V_{b}\right\rangle$ is maintained between $3 \mathrm{~V}$ and $5 \mathrm{~V}$, given the reference voltage $V_{\text {ref }}$ of the comparator LTC 1540 is $1.182 \mathrm{~V}$, the resistance of the voltage divider can be calculated from Equation (5.2):

$$
\left\{\begin{array}{l}
\frac{R 7+R 8}{R 6+R 7+R 8}=\frac{1.182}{3} \\
\frac{R 8}{R 6+R 7+R 8}=\frac{1.182}{5}
\end{array}\right.
$$

Therefore R6, R7 and R8 are chosen to be $3.83 \mathrm{M} \Omega, 1 \mathrm{M} \Omega$ and $1.5 \mathrm{M} \Omega$ respectively.

\subsection{Electronic Circuit Test}

\subsubsection{Test with the energy harvester on an hydraulic load frame}

The KEH circuit designed and analyzed above is tested on the KEH container designed in Chapter 3 under a hydraulic load frame. In this test, the hydraulic load frame's loading force is set at $1600 \mathrm{~N}$ with the frequency of $1 \mathrm{~Hz}$, which is the same as that in the open circuit voltage test under the hydraulic load frame in Chapter 3.

From Equation (3.38), the open circuit voltage peak of the KEH contrainer is $199 \mathrm{~V}$. Figure 5.2 shows that the piezoelectric voltage peaks at around $120 \mathrm{~V}$ and then discharges to the bucket capacitor through an inductor as designed. It should be mentioned that in each load there are two voltage peaks. The piezoelectric voltage peak is lower than the open circuit voltage because a part of the piezoelectric energy is shunted to the zero-slope peak detection circuit. Figure 5.2 also illustrates the voltage peak detection signal, which drops below zero whenever the piezoelectric voltage peaks and starts to decrease. 

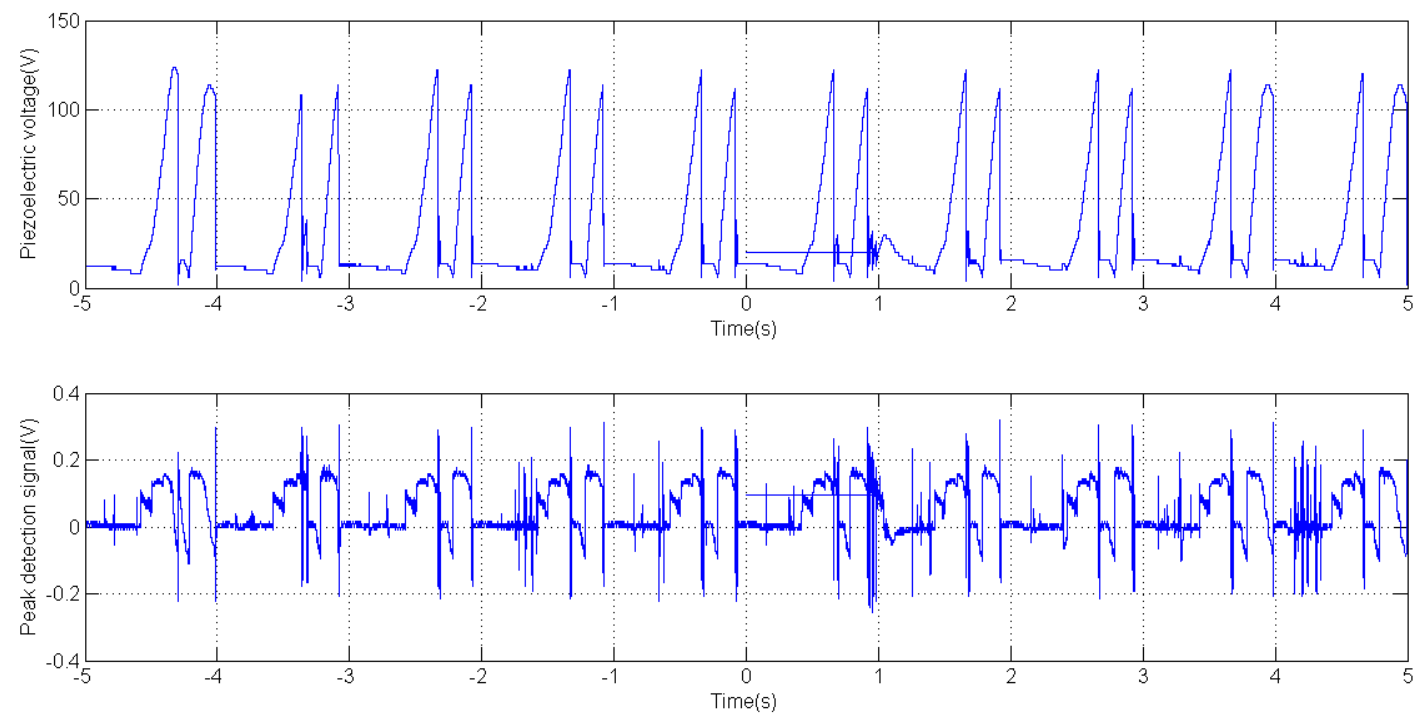

Figure 5.2 Piezoelectric voltage and peak detection signal.

Figure 5.3 illustrates the charging process of a bucket capacitor of $100 \mu \mathrm{F}$ during the test. The KEH container is compressed for 10 times within 10 seconds and the voltage of the bucket capacitor rises from $0.6 \mathrm{~V}$ to $11.4 \mathrm{~V}$. Therefore the energy increment of the bucket capacitor in each load $E_{M T S}$ is:

$$
\begin{aligned}
E_{\text {MTS }}= & \frac{1}{2} \times 100 \times 10^{-6} \times\left(11.4^{2}-0.6^{2}\right) \times \frac{1}{10} \\
& =0.648 \times 10^{-3} \mathrm{~J}=0.648 \mathrm{~mJ}
\end{aligned}
$$




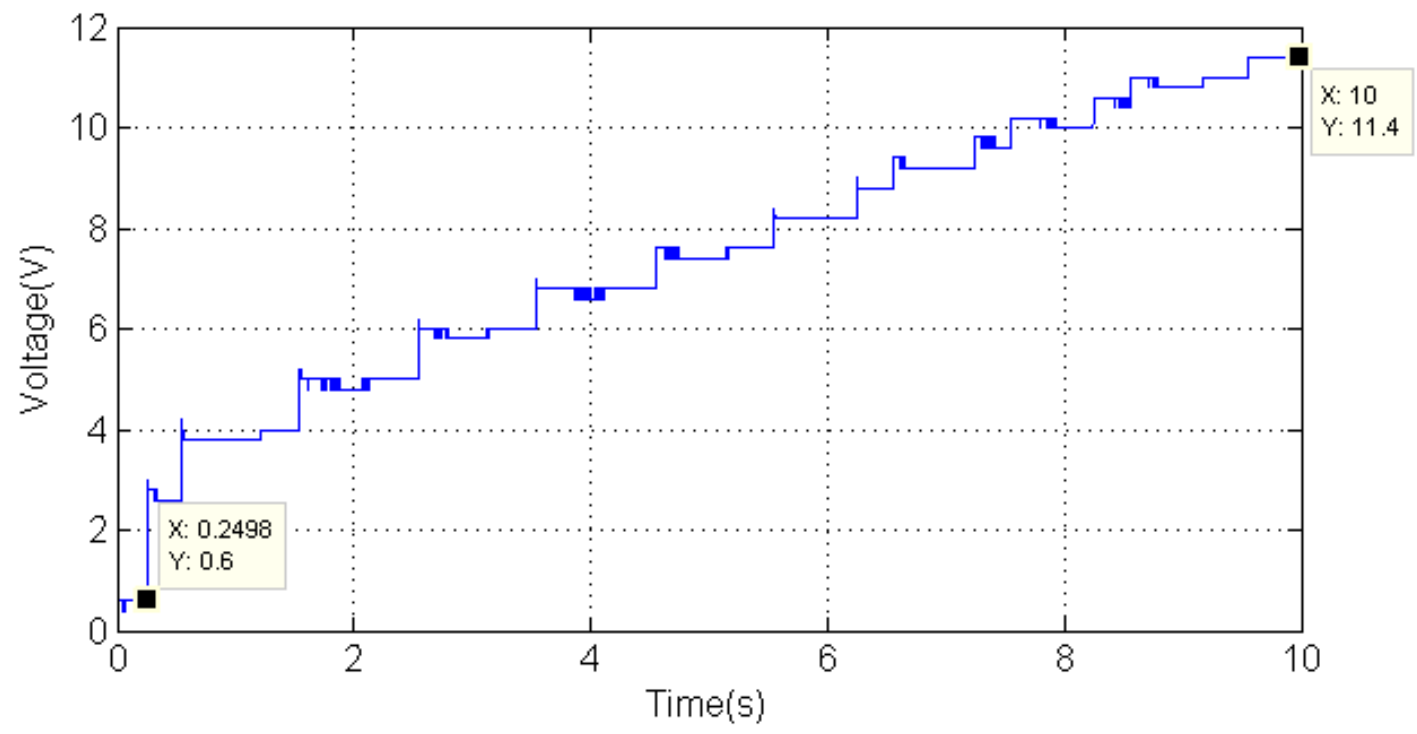

Figure 5.3 Charging process of a $100 \mu \mathrm{F}$ capacitor.

For the KEH container, given that the two open circuit voltage peaks of each load are 199 $\mathrm{V}$, and its piezoelectric capacitance is $7.4 \mathrm{nF} \times 18=133.2 \mathrm{nF}$. Then the available piezoelectric energy from the KEH container $E_{p z c}$ during each load is:

$$
E_{p z c}=\left(\frac{1}{2} \times 133.2 \times 10^{-9} \times 199^{2}\right) \times 2 \approx 5.27 \times 10^{-3} \mathrm{~J}=5.27 \mathrm{~mJ}
$$

Based on theoretical calculation, the efficiency $\eta_{M T S}$ of the circuit tested with the hydraulic load frame is:

$$
\eta_{M T S}=\frac{E_{M T S}}{E_{p Z c}}=\frac{0.648}{5.27}=12.3 \%
$$

In Appendix $C$, the updated piezoelectric power harvesting circuit in the form of a highfrequency forward converter from the MIT media lab is described. Based on theoretical calculation, the efficiency of their circuit is:

$$
\eta_{f w}=\frac{E_{f}}{E_{b}}=\frac{0.7042}{13.39} \approx 5.26 \%
$$


In comparison, the circuit based on the SSDCI topology has more than twice the efficiency of the high-frequency forward converter based on the SECE topology from the MIT media lab.

\subsubsection{Test on the KEH speed bump}

The KEH circuit is also tested on the speed bump embedded with a KEH container. In this test, the test vehicle is the same one that was used in the speed bump open circuit voltage test in Chapter 3, and the front wheel of the vehicle rolls over the speed bump back and forth for five times. From Equation (3.39), the open circuit voltage peak of the speed bump is $234 \mathrm{~V}$. Figure 5.4 shows that the piezoelectric voltage peaks between 100 $\mathrm{V}$ to $150 \mathrm{~V}$ and then discharges, and illustrates the voltage peak detection signal below.
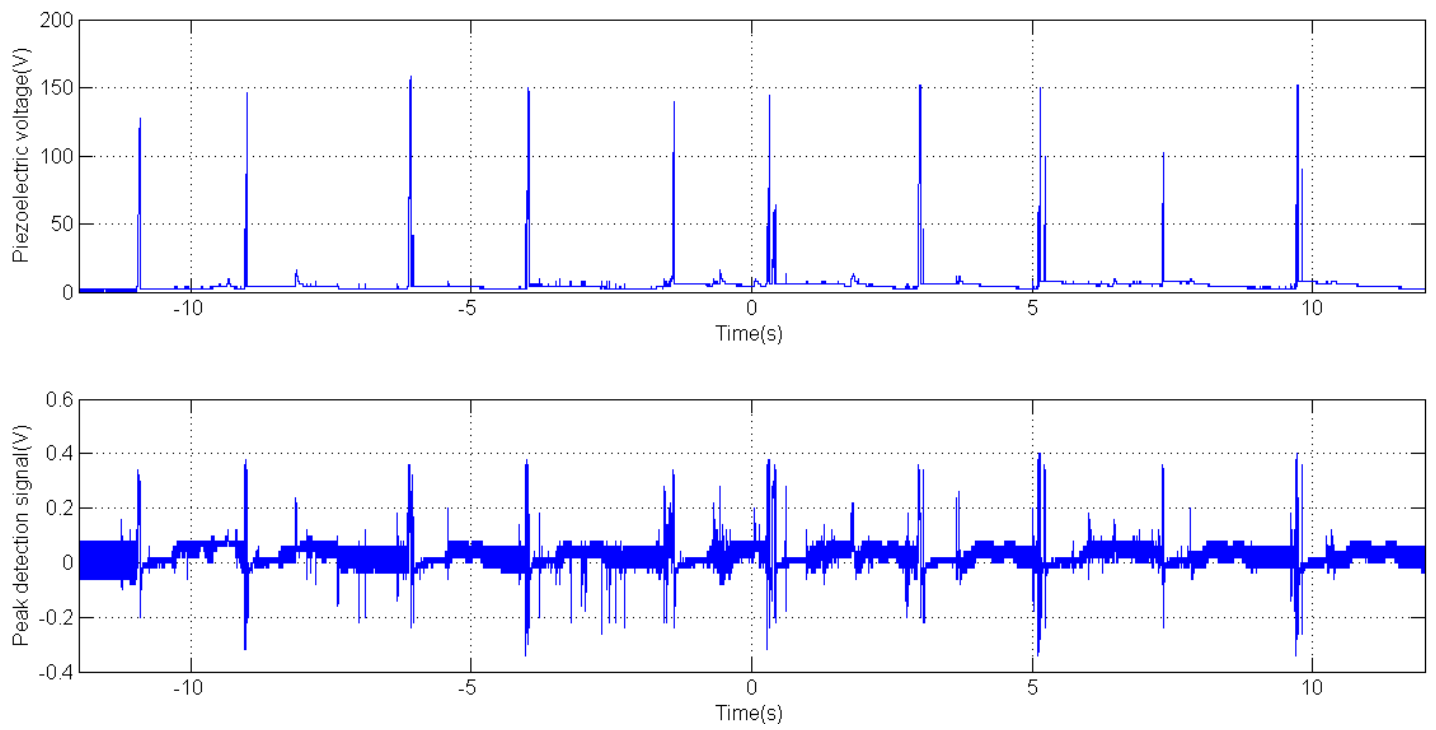

Figure 5.4 Piezoelectric voltage and peak detection signal. 
Figure 5.5 shows the charging process of a bucket capacitor of $100 \mu \mathrm{F}$ during the test. The voltage of the bucket capacitor increases from $0.16 \mathrm{~V}$ to $8.24 \mathrm{~V}$ under the wheel load of 10 times. Therefore the energy increment during each wheel load $E_{w h e e l}$ is calculated:

$$
\begin{gathered}
E_{\text {wheel }}=\frac{1}{2} \times 100 \times 10^{-6} \times\left(8.24^{2}-0.16^{2}\right) \times \frac{1}{10} \\
=0.394 \times 10^{-3} \mathrm{~J}=0.394 \mathrm{~mJ}
\end{gathered}
$$

For the KEH speed bump, given that the two open circuit voltage peaks of each load are $234 \mathrm{~V}$, and its piezoelectric capacitance is $7.4 \mathrm{nF} \times 20=148 \mathrm{nF}$. Then the available piezoelectric energy from the KEH speed bump $E_{p z s}$ during each load is:

$$
E_{p z S}=\left(\frac{1}{2} \times 148 \times 10^{-9} \times 234^{2}\right) \times 2 \approx 8.1 \times 10^{-3} \mathrm{~J}=8.1 \mathrm{~mJ}
$$

Given that the open circuit voltage peak of the speed bump is $234 \mathrm{~V}$, the theoretical efficiency $\eta_{\text {wheel }}$ of the circuit tested with speed bump can be calculated:

$$
\eta_{\text {wheel }}=\frac{E_{\text {wheel }}}{E_{p z s}}=\frac{0.394}{8.1} \approx 4.86 \%
$$




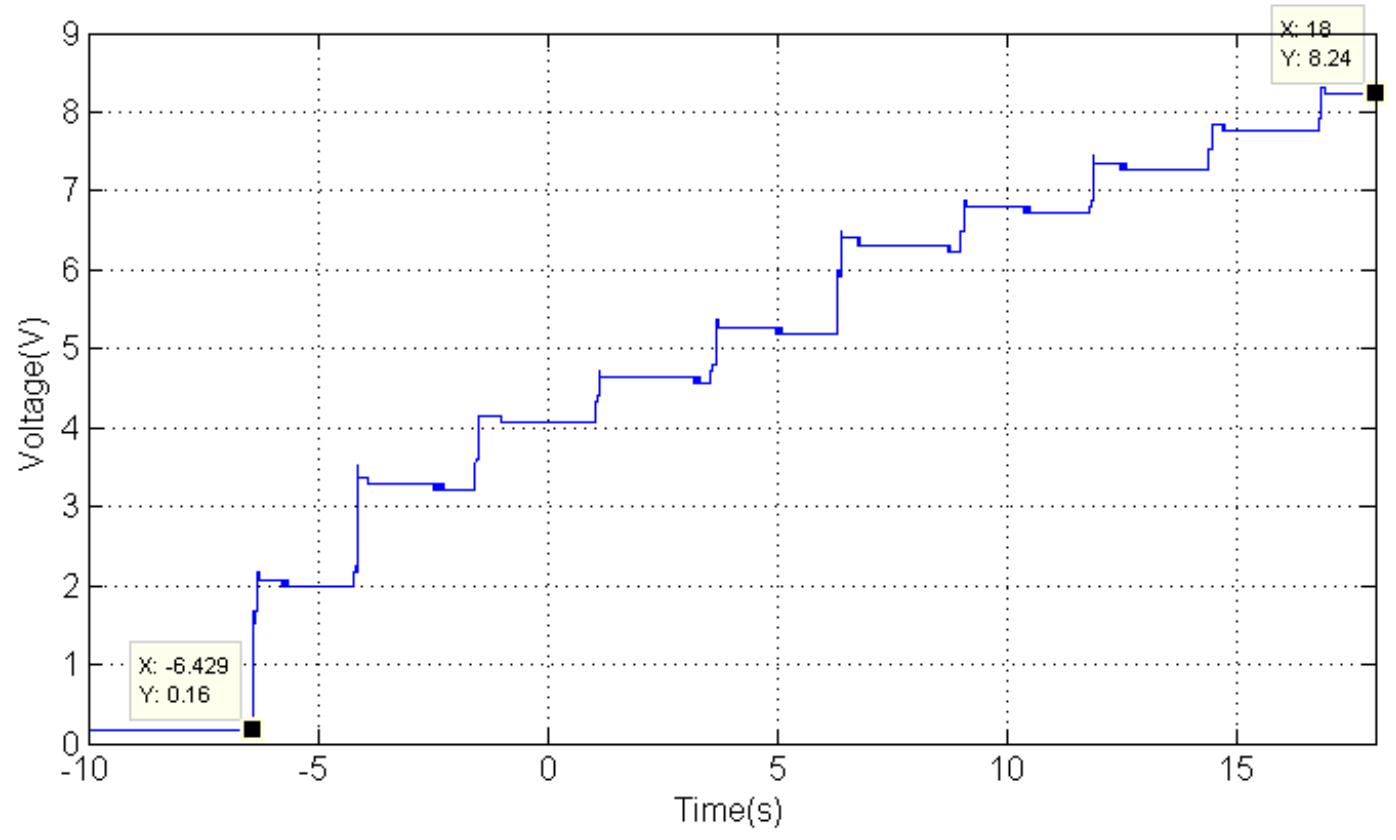

Figure 5.5 Charging process of a bucket capacitor of $100 \mu \mathrm{F}$.

In comparison, the hydraulic load frame charging test has an efficiency of $12.28 \%$, while the vehicle load charging test has a lower efficiency of $4.86 \%$. This is due to the fact that the load from the hydraulic load frame is applied vertically and every piezoelectric Cymbal unit is compressed and released simultaneously. However, for the KEH speed bump, the wheel load on the floating plate of the embedded container can make the plate tilt as the wheel rolls over the container, which will affect the total amount of harvested energy because the circuit will start discharging the piezoelectric capacitor when the second row of the piezoelectric units in the speed bump are not fully loaded.

Assuming that the traffic flowrate on one road is 1 vehicle per second, and each vehicle applies two loads (front wheel and back wheel) to the Cymbal array container, then the generated power is $0.788 \mathrm{~mW}$. 


\section{Chapter: Conclusions and Future work}

\subsection{Conclusions}

In this thesis, the design of a SRKEHS and its testing results are presented. The design work includes two parts: the KEHT and the KEH circuit.

In the design of the roadway KEHT, different kinds of KEHTs are compared and the PZT "Cymbal" transducer is chosen due to its larger contact surface, higher stability under loading and fabrication simplicity. A more comprehensive FEA simulation is performed on the "Cymbal" transducer to find its optimal geometry. A square container embedded with a $3 \times 3$ "Cymbal" array is designed and tested under a hydraulic load frame. Then a rectangular container embedded with a $2 \times 5$ "Cymbal" array is designed and tested with a speed bump under the wheel load of a test vehicle.

In the design of the KEH circuit, the SSDCI topology is chosen out of seven KEH circuit topologies after individual analysis and comparison about their harvested energy during each load with various rectified voltages. Based on the SSDCI circuit topology, a new piezoelectric KEH is designed and tested with both the $3 \times 3$ "Cymbal" array under the hydraulic load frame, and then a $2 \times 5$ "Cymbal" array embedded in the speed bump.

Based on the FEA analysis and test results, the following conclusions can be drawn:

1. Each Cymbal transducer is able to generate electric charges safely under a longitudinal load of $180 \mathrm{~N}$ with a corresponding displacement of $0.15 \mathrm{~mm}$. The Cymbal 
array is proved to be able to harvest energy from a high-impact-load and low-frequency kinetic energy source. For the speed bump embedded with a $2 \times 5$ Cymbal array container, the piezoelectric open circuit voltage is $234 \mathrm{~V}$, and it is estimated that $0.788 \mathrm{~mW}$ can be harvested with a vehicle flowrate of 1 vehicle per second. By adjusting the number of its rows and columns, it can be customized to a wide array of applications.

2. The power harvesting circuit has a higher efficiency (12.28\%) than the second power harvesting circuit developed by MIT media lab [86].

3. While testing the charging circuit with the speed bump, the calculated efficiency is lower. This is due to the assumption that all the Cymbal transducers are loaded simultaneously when calculating the circuit efficiency of the speed bump test.

\subsection{Recommendations for Future work}

Future research work should be conducted in the following subjects:

1. The PZT material generates electric charges under tension and compression. My team member Matteo is doing research to integrate a preload to the Cymbal transducer in radial direction. In this way the Cymbal transducer is able to endure a larger load and generate more energy.

2. The open circuit voltage test circuit can be improved by using a high impedance voltage divider, and accordingly the effect of the input impedance of the oscilloscope probe can be minimized.

3. Due to the $2 \times 5$ Cymbal array design, the cover plate above will tilt as the wheel rolls over and the Cymbal transducers in the second row will not reach their voltage peak 
before they are discharged. A possible cover design improvement would be to have individual plate for each row of the Cymbal array, and the plates can be connected by hinges. In this way, the load on the first-row plate will not affect the load on the secondrow plate, and each row will reach their voltage peak sequentially.

4. In the designed power harvesting circuit, the Cymbal transducers' voltage peak is decreased by the current shunt to the capacitor $\mathrm{C} 1$, and therefore the efficiency of the circuit is decreased as well. To address this problem, a possible Cymbal array connection improvement would be to have only a small part of the Cymbal transducers (e.g. one in each row) connected before $\mathrm{C} 1$ and the others are connected right before the peak detection circuit; $\mathrm{C} 1$ and the peak detection circuit could be separated by a diode. This design idea has been used in the PZT cantilever, which has a sensing PZT layer and a harvesting PZT layer [87]. In this way, most of the Cymbal transducers are able to reach their open circuit voltage peak and the circuit efficiency could be higher.

5. Another exciting future work is to integrate the KEHS with low-power electronic devices. It can be used to activate LED lights in construction region or accident hot spots. Wireless sensor network (WSN) is also a promising application for this system. The EHLink $^{\mathrm{TM}}$ Energy Harvesting Wireless Node [88] from LORD MicroStrain ${ }^{\circledR}$ features an accelerometer, humidity sensor, temperature sensor, and signal conditioning for a Wheatstone bridge which is compatible with strain gauges, magnetic sensors, etc. For a 1 $\mathrm{K} \Omega$ Wheatstone bridge with a data rate of 1 sample/second, the power consumption is only $0.24 \mathrm{~mW}$, which can be readily supplied by the KEHS. 


\section{Appendices}

\section{Appendix A Endcap die design}

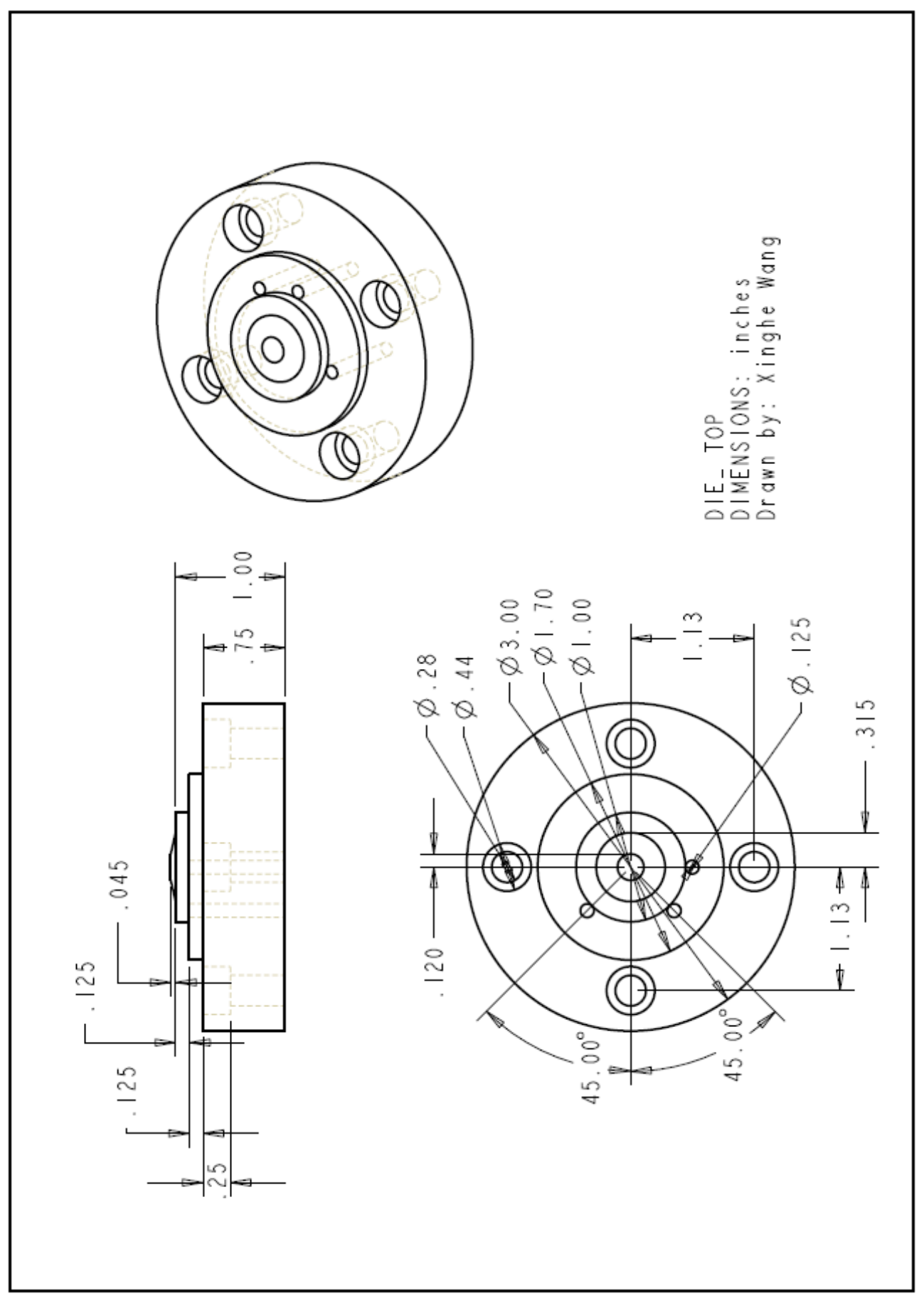

Figure A.1 Endcap die top part. 


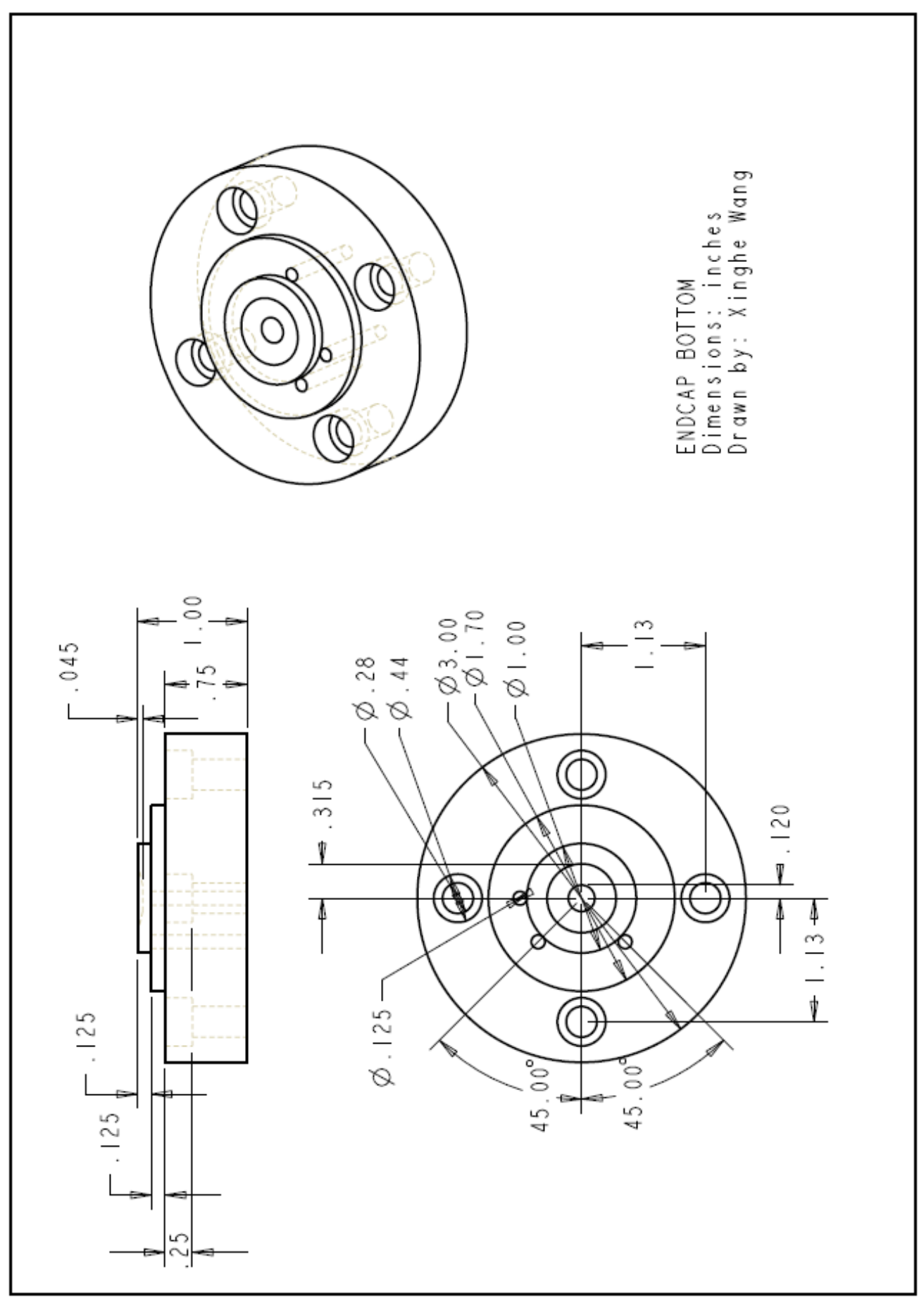

Figure A.2 Endcap die bottom part. 


\section{Appendix B Cymbal array container design}

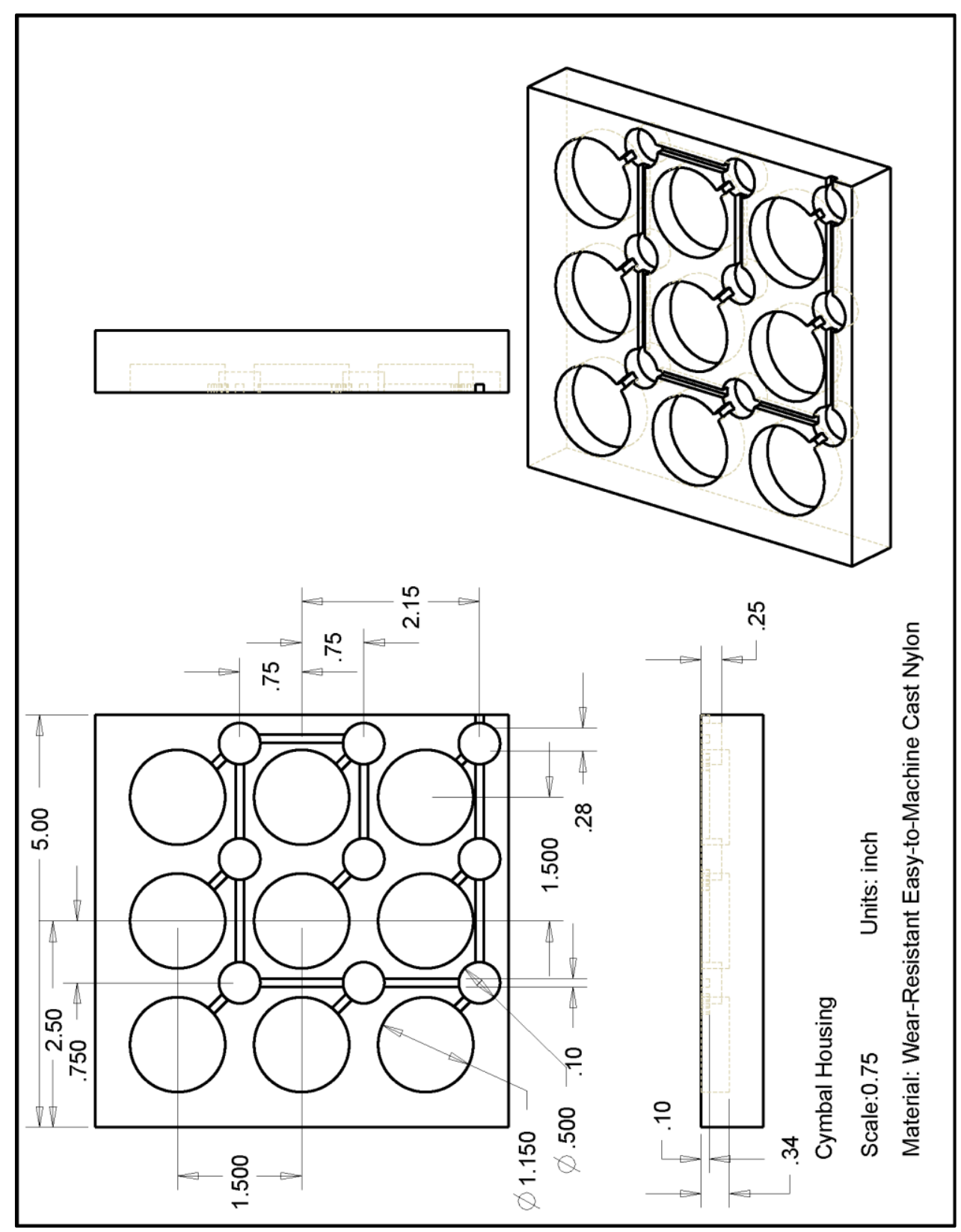

Figure B.1 The $3 \times 3$ "Cymbal” array container for load frame test. 


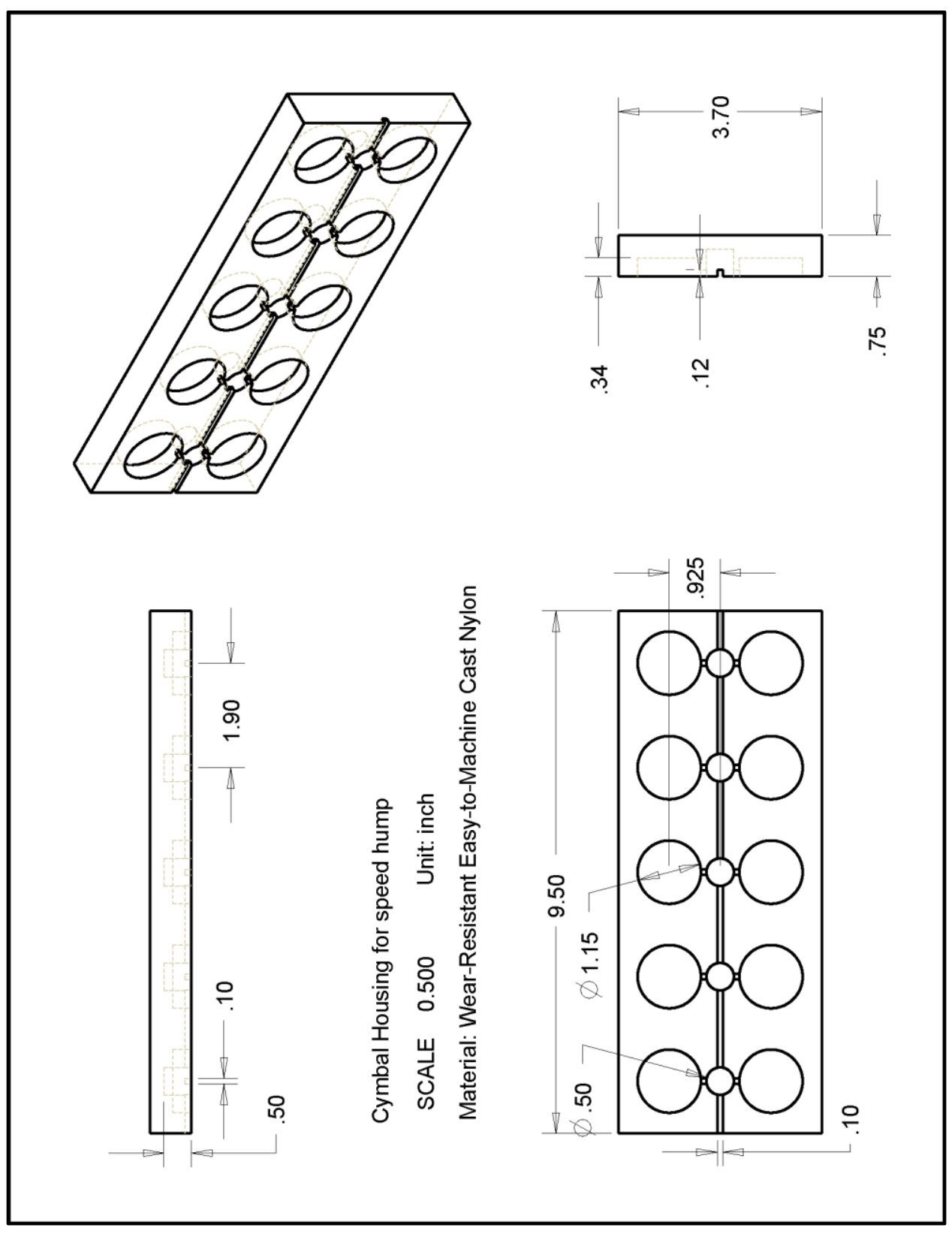

Figure B.2 The $2 \times 5$ "Cymbal” array container for vehicle test. 


\section{Appendix C Functional Description and Efficiency of the MIT Piezoelectric Power harvesting circuit}

The MIT designed a piezoelectric KEH circuit using the SECE topology in the form of a forward converter (illustrated in Figure D.1); the circuit is used to harvest energy from a PZT embedded shoe $[86,89]$.

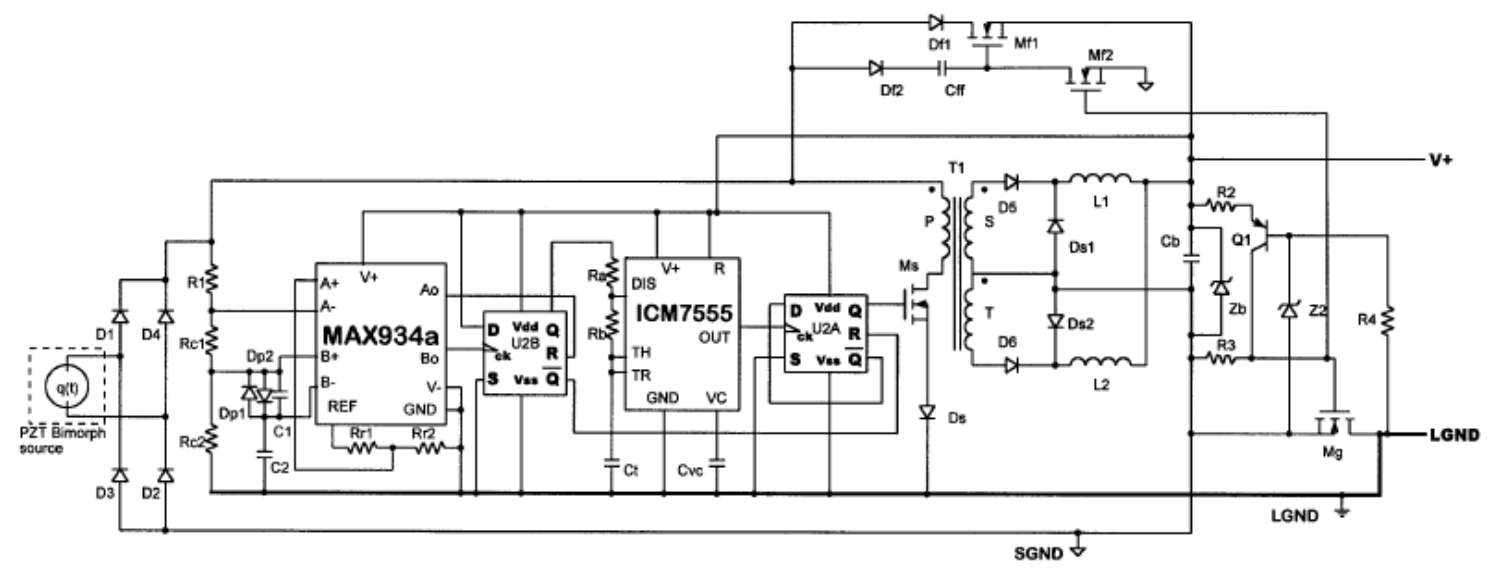

Figure D.1 Piezoelectric Power harvesting circuit from MIT media lab [86].

This power harvesting circuit has two ground buses: source ground (SGND) and load ground (LGND). As the system is in "cold condition" (i.e. the bucket capacitor $C_{b}$ is below a certain voltage threshold), SGND and LGND are disconnected by the MOSFET $M_{g}$, and $C_{b}$ is charged directly from the PZT Bimorph through $D_{f 2}, C_{f f}$ and $M_{f 1}$. When the voltage of $C_{b}$ is high enough, the MOSFET $M_{f 2}$ and $M_{g}$ are activated and the zero-peak slope detection and switching function of this circuit is able to work properly with the power provided by $C_{b}$. The 7555 timer switches the MOSFET $M_{s}$ on and off at a 
frequency of $25 \mathrm{kHz}$ when the peak comes. Therefore the bucket capacitor $C_{b}$ is charged by the source through a step-down transformer $\mathrm{T} 1$.

Figure D. 2 shows that the voltage of the $300 \mu \mathrm{F}$ bucket capacitor increases from $0 \mathrm{~V}$ to 13 $V$ after 36 loads of foot step. Therefore the energy increment during each foot step $E_{f}$ is:

$$
\begin{aligned}
E_{f} & =\frac{1}{2} \times 300 \times 10^{-6} \times\left(13^{2}-0\right) \times \frac{1}{36} \\
& =0.7042 \times 10^{-3} \mathrm{~J}=0.7042 \mathrm{~mJ}
\end{aligned}
$$

\section{Bucket Voltage With 36 Steps using Bimorph}

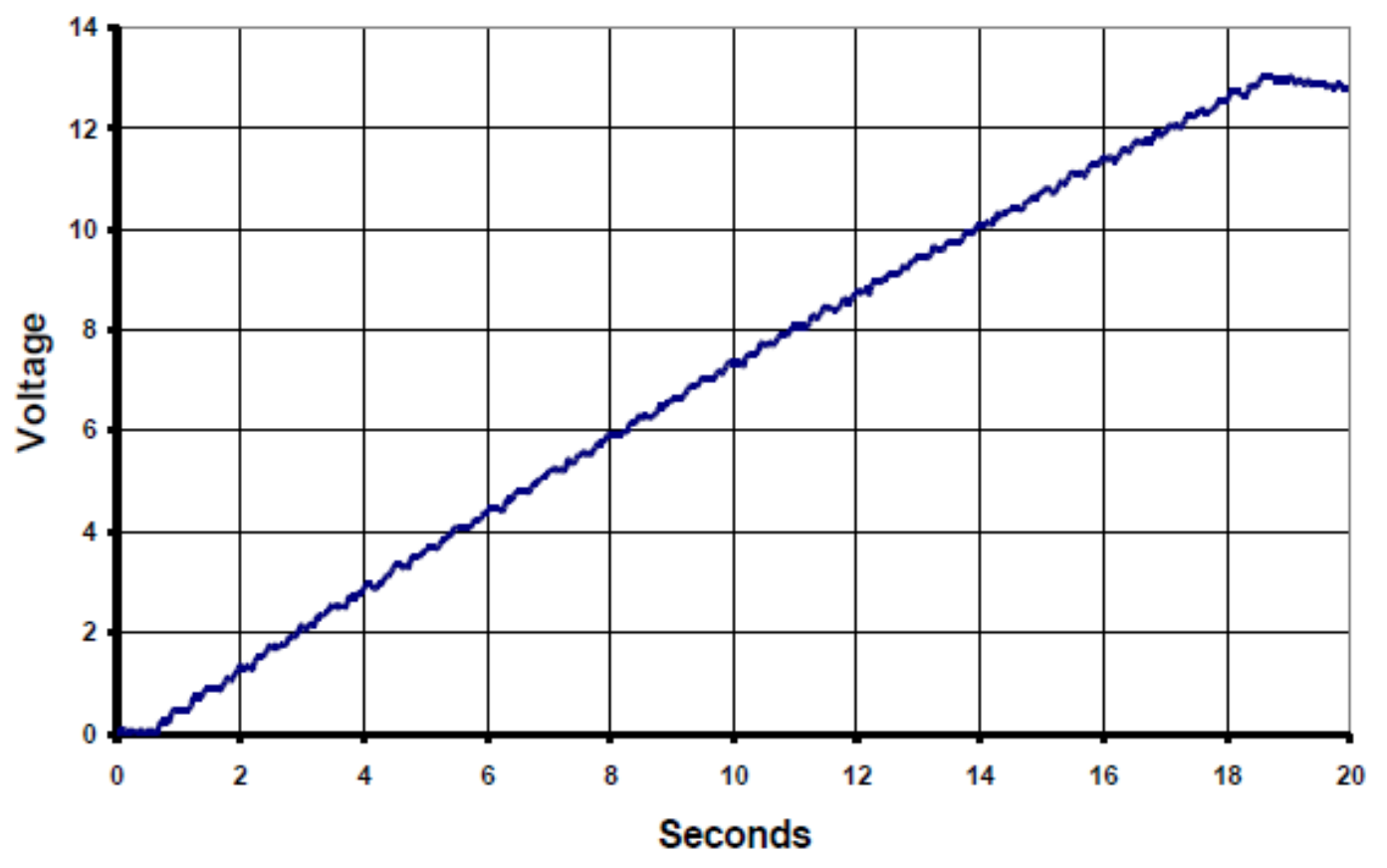

Figure D.2 300 $\mu$ F bucket capacitor charging process under 36 steps. [86] 
For the PZT bimorph source, the open circuit voltage peak of either compression or release is $306 \mathrm{~V}$, and its piezoelectric capacitance is $143 \mathrm{nF}$. Then the available piezoelectric energy from the PZT bimorph source $\left\langle E_{f s}\right\rangle$ during each load is:

$$
E_{b}=\left(\frac{1}{2} \times 143 \times 10^{-9} \times 306^{2}\right) \times 2 \approx 13.39 \times 10^{-3} \mathrm{~J}=13.39 \mathrm{~mJ}
$$

Based on theoretical calculation, the efficiency $\eta_{f w}$ of the circuit tested with the KEH footwear is:

$$
\eta_{f w}=\frac{E_{f}}{E_{b}}=\frac{0.7042}{13.39} \approx 5.26 \%
$$




\section{Bibliography}

[1] S.Monfray, O.Puscasu and G. Savelli, "Innovative thermal energy harvesting for zero power electronics," Silicon Nanoelectronics Workshop, pp. 1-4, 2012.

[2] S. Mekhilef and R. Saidur, "A review on solar energy use in industries," Renewable and Sustainable Energy Reviews, vol. 15, no. 4, pp. 1777-1790, 2011.

[3] G. D. Szarka, B. H. Stark and S. G. Burrow, "Review of Power Conditioning for Kinetic Energy Harvesting Systems," IEEE Transactions on Power Electronics, vol. 27, no. 2,2012 .

[4] A. Harb, "Energy harvesting: State-of-the-art," Renewable Energy, vol. 36, pp. 2641-2654, 2011.

[5] J. Paradiso and T. Starner, "Energy Scavenging for Mobile and Wireless Electronics," IEEE Computer Society, pp. 18-27, 2005.

[6] S. Arms, C. Townsend, D. Churchill, J. Galbreath and S. Mundell, "Power Management for Energy Harvesting Wireless Sensors," TELSIKS '09, pp. 65-72, 2009.

[7] S. W. Arms, C. P. Townsend and D. L. Churchill, "Energy Harvesting Wireless Sensors," in Energy Harvesting Technologies, Boston, MA, Springer US, 2009, pp. 195-208.

[8] "The World Factbook," CIA, 23 June 2014. [Online]. Available: https://www.cia.gov/library/publications/the-worldfactbook/wfbExt/region_noa.html. 
[9] D. Halliday, R. Resnick and J. Walker, Fundamental of Physics, 2014.

[10] S. P. Beeby and T. O'Donnell, "Electromagnetic Energy Harvesting," in Energy Harvesting Technology, Boston, MA, Springer US, 2009, pp. 129-161.

[11] J. Y. Hayashida, "Unobtrusive Integration of Magnetic Generator Systems into Common Footwear," Cambridge, 2000.

[12] L. Kemball-Cook and P. Tucker, "Energy Harvesting". US Patent 2013/0068047, May 2013.

[13] C. Ting, D. Tsai and C. Hsiao, "Developing a mechanical roadway system for waste energy capture of vehicles and electric generation," Applied Energy, vol. 92, pp. 1-8, 2012.

[14] S. Ashley, "Artificial Muscles," Scientific American sp, vol. 18, no. 1, pp. 64-71, 2008.

[15] R. Kornbluh and R. Pelrine, "Electroelastomers: Applications of Dielectric Elastomer Transducers for Actuation, Generation and Smart Structures," Smart Structures and Materials 2002: Industrial and Commercial Applications of Smart Structures Technologies, March 2002.

[16] W. Roentgen, "About the Changes in Shape and Volume of Dielectrics Caused by Electricity," Ser. Annual Physics and Chemistry, pp. 771-786, 1880.

[17] T. Vu-Cong, C. Jean-Mistral and A. Sylvestre, "Electrets substituting external bias voltage in dielectric elastomer generators: application to human motion," SMART MATERIALS AND STRUCTURES, vol. 22, no. 2, p. 025012, 2013.

[18] T. McKay, B. O’Brien, E. Calius and I. Anderso, "Self-priming dielectric elastomer 
generators," SMARTMATERIALS AND STRUCTURES, 2010.

[19] T. McKay, B. O’Brien and E. Calius, "Realizing the Potential of Dielectric Elastomer Generators," Electroactive Polymer Actuators and Devices (EAPAD), vol. 7976, 2011.

[20] T. McKay, B. O’Brien and E. Calius, "An integrated, self-priming dielectric elastomer generator," APPLIED PHYSICS LETTERS, 2010.

[21] R. D. Kornbluh, R. Pelrine, H. Prahlad, A. Wong-Foy and B. McCoy, "From boots to buoys: Promises and challenges of dielectric elastomer energy harvesting," Electroactive Polymer Actuators and Devices (EAPAD) 2011,, 2011.

[22] S. Chiba, M. Waki, R. Kornbluh and R. Pelrine, "Innovative Power Generators for Energy Harvesting Using Electroactive Polymer Artificial Muscles," Electroactive Polymer Actuators and Devices (EAPAD), 2008.

[23] A. Messineo, A. Alaimo, M. Denaro and D. Ticali, "Piezoelectric Bender Transducers for Energy Harvesting Applications," Energy Procedia, vol. 14, pp. 3944, 2012.

[24] H. Zhao, J. Yu and J. Ling, "Finite element analysis of Cymbal piezoelectric transducers for harvesting energy from asphalt pavement," Journal of the Ceramic Society of Japan, vol. 118, no. 1382, pp. 909-915, 2010.

[25] L. Edery-Azulay, "Innowattech: Harvesting Energy and Data A stand alone technology," Tel Aviv, 2010.

[26] H. Abramovich and C. Milgrom, "MULTI-LAYER PIEZOELECTRIC GENERATOR". US Patent 8,278,800 B2, 2 Oct 2012. 
[27] H. Abramovich, E. Harash and C. Milgrom, "ENERGY HARVESTING FROM AIRPORT RUNWAY". US Patent 2009/0195124 A1, 6 Aug 2009.

[28] H. Abramovich and E. Harash, "POWER HARVESTING FROM RAILWAY; APPARATUS, SYSTEMAND METHOD". US Patent 7,812,508 B2, 12 Oct 2010.

[29] I. Moskowitz, "Israel's Innowattech to Provide Renewable Energy for Highway $\begin{array}{llll}\text { Signs in } \quad \text { Italy," } & \text { [Online]. }\end{array}$ http://www.greenprophet.com/2010/05/israel\%E2\%80\%99s-innowattech-toprovide-renewable-energy-for-highway-signs-in-italy/.

[30] J. Antaki and G. Bertocci, "A Gait-Powered Autologous Battery Charging System for Artificial Organs," ASAIO J., vol. 41, no. 3, 1995.

[31] J. Kymissis, C. Kendall, J. Paradiso and N. Gershenfeld, "Parasitic power harvesting in shoes," Digest of Papers. Second International Symposium on Wearable Computers, pp. 132-139, 1998.

[32] L. Moro and D. Benasciutti, "Harvested power and sensitivity analysis of vibrating shoe-mounted piezoelectric cantilevers," SMART MATERIALS AND STRUCTURES, vol. 19 , no. 11, p. 115011, 2010.

[33] C. Blades and E. Kearney, "Asphalt Paving Principles," New York LTAP Center, New York, 2004.

[34] H. ZHAO, J. LING and J. YU, "A comparative analysis of piezoelectric transducers for harvesting energy from asphalt pavement," Journal of the Ceramic Society of Japan, vol. 120, no. 1404, pp. 317-323, 2012.

[35] "Typical Values of Young's Elastic Modulus and Poisson's Ratio for Pavement 
Materials," Cornell University, [Online]. Available: ftp://www.clrp.cornell.edu/CDOT/Handouts/4c\%20\%20Materials\%20Table.pdf. [Accessed 2014].

[36] K. Lubitz, C. Schuh, T. Steinkopff and A. Wolff, "Material Aspects for Reliability and Life Time of PZT Multilayer Ceramics," in Piezoelectric Materials in Devices, Lausanne, N. Setter, 2002, pp. 183-194.

[37] R. Pelrine, R. Kornbluh and Q. Pei, "High-Speed Electrically Actuated Elastomers with Strain Greater Than 100\%," American Association for the Advancement of Science, vol. 287, pp. 836-839, 2000.

[38] R. Kornbluh and R. Pelrine, "Dielectric elastomer produces a strain of 380\%," Worldwide electroactive polymer EAP Newsletter, 2000.

[39] R. S. Joseph, "Energy Scavenging for Wireless Sensor Nodes with a Foucus on Vibration to Electricity Conversion," THE UNIVERSITY OF CALIFORNIA,BERKELEY, 2003.

[40] "Americanpiezo," [Online]. Available: http://www.americanpiezo.com/. [Accessed 2014].

[41] "Measurement Specialties," [Online]. Available: http://www.measspec.com/downloads/Piezo_Technical_Manual.pdf. [Accessed 2014].

[42] J. Curie and P. Curie, "Development, via compression, of electric polarization in hemihedral crystals with inclined faces," Bulletin de la Société minérologique de France, vol. 3, pp. 90-93, 1880.

[43] Piezoelectric Ceramics Principles and Applications, Apc International, Ltd., 2011. 
[44] B. Richter, J. Twiefel and JorgWallaschek, "Piezoelectric Equivalent Circuit Models," in Energy Harvesting Technologies, Springer Science+Business Media, 2009, p. 107.

[45] R. Muniandy, D. Moazami, H. Hamid and S. Hassim, "Characterization of Effective Tire Contact Area for Various Tread Patterns," Instrumentation Science and Technology, vol. 42, pp. 15-26, 2014.

[46] O. M. o. Transportation, "Ontario Driver's Handbook," 2014. [Online]. Available: http://www.mto.gov.on.ca/english/dandv/driver/handbook/section2.2.6.shtml.

[47] M.S.Vijaya, "Engineering Applications of Piezoelectric Materials," in Piezoelectric Materials and Devices, CRC Press, 2013, p. 57.

[48] G. H. Haertling, "Compositional Study of PLZT Rainbow Ceramics for Piezo Actuators," Int. Symp. on Applications of Ferroelectrics, pp. 313-318, 1994.

[49] K. M. Mossi, G. V. Selby and R. G. Bryant, "Thin-layer composite unimorph ferroelectric driver and sensor properties," Materials Letters, vol. 35, no. 1-2, pp. 3949, 1997.

[50] "FACE PRODUCT PORTFOLIO," [Online]. $\quad$ Available: http://www.faceinternational.com/. [Accessed 2014].

[51] R. E. Newnham, A. Dogan and Q. C. Xu, "FlEXTENSIONAL "MOONIE" ACTUATORS," Ultrasonics Symposium, vol. 1, pp. 509-513, 1993.

[52] A. Dogan, K. Uchino and R. E. Newnham, "Composite Piezoelectric Transducer with Trancated Conical Endcaps "Cymbal"," Ultrasonics, Ferroelectrics, and Frequency Control, IEEE Transactions, vol. 44, no. 3, pp. 597 - 605, 1997. 
[53] K. Hyeoung Woo, A. Batra, S. Priya and K. Uchino, "Environment, Energy Harvesting Using a Piezoelectric "Cymbal” Transducer in Dynamic Environment," Japanese Journal of Applied Physics, vol. 43, no. 9A, pp. 6178-6183, 2004.

[54] H. W. Kim, S. Priya, K. Uchino and R. E. Newnham, "Piezoelectric Energy Harvesting under High Pre-Stressed Cyclic Vibrations," Journal of Electroceramics, vol. 15, no. 1, pp. 27-34, 2005.

[55] K. Uchino and T. ishii, "Energy Flow Analysis in Piezoelectric Energy Harvesting Systems," Ferroelectrics, vol. 400, no. 1, pp. 305-320, 2010.

[56] H. Kim, S. Priya and K. Uchino, "Modeling of Piezoelectric Energy Harvesting Using Cymbal Transducers," Jpn. J. Appl. Phys., vol. 45, no. 7, pp. 5836-5840, 2006.

[57] H. Kim, S. Priya, H. Stephanou and K. Uchino, "Consideration of Impedance Matching Techniques for Efficient Piezoelectric Energy Harvesting," IEEE TRANSACTIONS ON ULTRASONICS, FERROELECTRICS, AND FREQUENCY CONTROL, vol. 54, no. 9, pp. 1851-1859, 2007.

[58] H. W. Kim, A. Batra, S. Priya, K. Uchino, D. Markley, R. E. Newnham and H. F. Hofmann, "Energy Harvesting Using a Piezoelectric "Cymbal" Transducer in Dynamic Environment," Japanese Journal of Applied Physics, vol. 43, no. 9A, pp. 6178-6183, 2004.

[59] Y. A. C. Ramadass, "An Efficient Piezoelectric Energy Harvesting Interface Circuit Using a Bias- Flip Rectifier and Shared Inductor," IEEE Journal, vol. 45, no. 1, pp. 189-204, 2010. 
[60] G. K. Ottman, H. F. Hofmann, A. C. Bhatt and G. A. Lesieutre, "Adaptive Piezoelectric Energy Harvesting Circuit for Wireless Remote Power Supply," IEEE TRANSACTIONS ON POWER ELECTRONICS, vol. 17, no. 5, pp. 669 - 676, 2002.

[61] "Knowledge Center, FAQ," APC International, Ltd., 2014. [Online]. Available: https://www.americanpiezo.com/knowledge-center/faq.html.

[62] "Stack Actuators," APC International, Ltd., 2014. [Online]. Available: https://www.americanpiezo.com/uncategorised/stack-actuators.html.

[63] J. d. C. S. Román, "Experiments on Epoxy, Polyurethane and ADP Adhesives," Composite Construction Laboratory, 2005.

[64] D. Aydin, "Flextensional "moonie and cymbal" actuators," Thesis, The Pennsylvania State University, 1994.

[65] McMASTER-CARR. [Online]. Available: http://www.mcmaster.com/.

[66] J. Zhang, W. Hughes, R.J.Meyer and K. Uchino, "Cymbal array: a broad band sound projector," Ultrasonics, vol. 37, pp. 523-529, 2000.

[67] A. Dogan, K. Uchino and R. E. Newnham, "Composite Piezoelectric Transducer with Truncated Conical Endcaps "Cymbal"," IEEE TRANSACTIONS ON ULTRASONICS, FERROELECTRICS, AND FREQUENCY CONTROL, vol. 44, no. 3, pp. 597-605, 1997.

[68] J. Liu, H. Fang and Z. Xu, "A MEMS-based piezoelectric power generator array for vibration energy harvesting," Microelectromics, vol. 39, pp. 802-806, 2008.

[69] W. Wang, T. Yang, X. Chen and X. Yao, "Vibration Energy Harvesting Using a Piezoelectric Circular Diaphragm Array," IEEE TRANSACTIONS ON 
ULTRASONICS, FERROELECTRICS, AND FREQUENCY CONTROL, vol. 59, no. $9,2012$.

[70] S. R. Platt, S. Farritor and H. Haider, "On Low-Frequency Electric Power Generation With PZT Ceramics," IEEE/ASME Transactions on Mechatronics, vol. 10, no. 2, pp. 240-252, 2005.

[71] M. J. Cuan and W. H. Liao, "Characteristics of Energy Storage Devices in Piezoelectric Energy Harvesting Systems," Journal of Intelligent Material Systems and Structures, vol. 19, no. 6, pp. 671-680, 2007.

[72] F. I. Simjee and P. H. Chou, "Efficient Charging of Supercapacitors for Extended Lifetime of Wireless Sensor Nodes," IEEE TRANSACTIONS ON POWER ELECTRONICS, vol. 23, no. 3, pp. 1526-1536, 2008.

[73] D. Guyomar and M. Lallart, "Recent Progress in Piezoelectric Conversion and Energy Harvesting Using Nonlinear Electronic Interfaces and Issues in Small Scale Implementation," Micromachines, vol. 2, no. 4, pp. 274-294, 2011.

[74] A. Badel, A. Benayad, E. Lefeuvre, L. Lebrun, C. Richard and D. Guyomar, "Single Crystals and Nonlinear Process for Outstanding Vibration-Powered Electrical Generators," IEEE TRANSACTIONS ON ULTRASONICS, FERROELECTRICS, AND FREQUENCY CONTROL, vol. 53, no. 4, pp. 673-684, 2006.

[75] N. M. Roscoe and M. D. Judd, "Optimization of Voltage Doublers for Energy Harvesting Applications," IEEE SENSORS JOURNAL, vol. 13, no. 12, pp. 49044911, 2013.

[76] E. Lefeuvre, A. Badel, C. Richard, L. Petit and D. Guyomar, "A comparison 
between several vibration-powered piezoelectric generators for standalone systems," Sensors and Actuators A: Physical, vol. 126, no. 2, p. 405-416, 2006.

[77] A. Badel, D. Guyomar, E. Lefeuvre and C. Richard, "Efficiency Enhancement of a Piezoelectric Energy Harvesting Device in Pulsed Operation by Synchronous Charge Inversion," Journal of Intelligent Material Systems and Structures, vol. 16, no. 10, pp. 889-901, 2005.

[78] S. Mehraen, S. Jagannathan and K. Corzine, "Energy harvesting using piezoelectric materials and high voltage scavenging circuitry," IEEE International Conference on Industrial Technology, pp. 1-8, 2008.

[79] A. Badel, D. Guyomar, E. Lefeuvre and C. Richard, "Piezoelectric Energy Harvesting using a Synchronized Switch Technique," Journal of Intelligent Material Systems and Structures, vol. 17, no. 8-9, pp. 831-839, 2006.

[80] W. J. Wu, A. M. Wickenheiser, T. Reissman and E. Garcia, "Modeling and experimental verification of synchronized discharging techniques for boosting power harvesting from piezoelectric transducers," SMART MATERIAL AND STRUCTURE, vol. 18 , no. 5, p. 055012, 2009.

[81] E. Lefeuvre, A. Badel, C. Richard and D. Guyomar, "Piezoelectric Energy Harvesting Device Optimization by Synchronous Electric Charge Extraction," JOURNAL OF INTELLIGENT MATERIAL SYSTEMS AND STRUCTURES, vol. 16, pp. 865-876, 2005.

[82] E. Lefeuvre, A. Badel, C. Richard, L. Petit and N. Guyomar, "OPTIMIZATION OF PIEZOELECTRIC ELECTRICAL GENERATORS POWERED BY RANDOM 
VIBRATIONS," DTIP of MEMS \& MOEMS, pp. 26-28, 2006.

[83] C. Richard, D. Guyomar, D. Audigier and H. Bassaler, "Enhanced semi passive damping using continuous switching of a piezoelectric device on an inductor," Proceedings of the SPIE, vol. 3989, pp. 288-299, 2000.

[84] A. Bade, G. Sebald, D. Guyomar, M. Lallart, E. Lefeuvre, C. Richard and J. Qiu, "Piezoelectric vibration control by synchronized switching on adaptive voltage sources: Towards wideband semi-active damping," Acoustical Society of America, vol. 119, pp. 2815-2825, 2006.

[85] M. Lallart, L. Garbuio, L. Petit, C. Richard and D. Guyomar, "Double synchronized switch harvesting (DSSH): a new energy harvesting scheme for efficient energy extraction," IEEE transactions on ultrasonics, ferroelectrics, and frequency control, vol. 55 , no. 10 , pp. $2119-30,2008$.

[86] N. S. Shenck, "A Demonstration of Useful Electric Energy Generation from Piezoceramics in a Shoe," Massachusetts Institute of Technology, 1999.

[87] N. M. pour, D. Zhu, R. N. Torah, A. D. T. Elliot, P. D. Mitcheson and S. P. Beeby, "A novel piezoelectric energy harvester designed for single-supply pre-biasing circuit," Journal of Physics: Conference Series, vol. 476, no. 1, p. 012134, 2013.

[88] "EH-Link ${ }^{\mathrm{TM}}$ Energy Harvesting Wireless Node," LORD MicroStrain, March 2015. [Online]. Available: http://www.microstrain.com/energy-harvesting/eh-link.

[89] N. S. Shenck and J. A. Paradiso, "Energy Scavenging With Shoe-Mounted Piezoelectrics," Micro, IEEE, vol. 21, no. 3, pp. 30-42, 2001. 